\title{
Leadership as Communicative Practice: The discursive construction of leadership and team identity in a New Zealand rugby team
}

by

Nicholas Andrew Wilson

A thesis submitted to the Victoria University of Wellington in fulfilment of the requirements for the degree of Doctor of Philosophy

Victoria University of Wellington

2011 


\begin{abstract}
In sports teams, the way in which leaders such as coaches and captains communicate with players is vital to the success of the team. However, despite extensive psychological and sociological research on sport, it has rarely been a site of linguistic research. Like many sports, rugby has many traditions and ideologies that influence the way in which teams form identities. This thesis explores the way in which leadership is enacted and group identity forged through communicative practice in a New Zealand rugby team. Using authentic interactions collected using an ethnographic methodology, an analysis is presented of how discourse strategies are negotiated within the team, establishing practices that signify membership of communities of practice (CofPs) and creating identities for individuals as leaders. Leadership discourse is itself viewed as a sociolinguistic practice and defines one of the CofPs within the team.
\end{abstract}

Using the concepts of front and back-stage (Goffman 1959; Richards 2006) to describe different conceptual spaces in which interactions occur, I suggest that discourse in the rugby team is a spatialised practice; the performance of a particular style of leadership constructs the space in which it takes place as public or private, with each contributing to an effective leadership performance. The construction of leadership identity is analysed in terms of stance and indexicality, linking locally constructed identities and discourse strategies to macro identity categories and socio-cultural ideologies. One of the ways in which this is examined is through the role of ritual and formulaic language in the team, showing that while communicative practice is negotiated in the back stage, in the front stage its performance serves to construct team identity while aiming to motivate the players. Furthermore, the structural nature of the game of rugby (i.e. players' positional requirements) is examined in relation to the different communicative strategies adopted by positionally segregated groups. It is suggested that these groups, although institutionally defined, create meaning for themselves as CofPs by negotiating a shared way of communicating in enacting their role in the team.

In sum, this research uses CofP theory to examine how leaders emerge through their linguistic practices. Furthermore, it locates leadership as a spatialised practice and examines how leaders influence the discursive construction of group identity. Finally, the analysis also makes a valuable contribution to the field of sociolinguistic research on sport, a small yet growing area. 


\section{Acknowledgements}

I am thankful for the support and guidance provided my two supervisors, Professor Janet Holmes and Dr Meredith Marra, whose mentoring and constant feedback on my writing has been absolutely crucial in steering my academic development. The speed at which they work coupled with a sometimes unnerving attention to detail has been a source of inspiration to the way in which I have approached my research. Thanks also to the supportive environment of LALS and the LALS thesis group in particular, who have provided moral support, in particular the various office mates who have all contributed in their own unique way to my $\mathrm{PhD}$ experience. Special thanks to Kieran for the being the "other sports linguist", to Brian for always being one step ahead of me, and to Ewa for introducing me to multimodality.

This thesis was completed with the aid of a Victoria University of Wellington Doctoral Assistantship, for which I am truly grateful. In addition I must thank the School of Linguistics and Applied Language Studies for providing me with part-time employment, a necessity in PhD life which often goes under-reported.

Thanks also to all the rugby people who participated in this research, without whom it literally would not have been possible.

I could not have completed this thesis however, without the support of my wife Fiona and the welcome distractions from work provided by our son, Ronan. Thank you. 


\section{Contents}

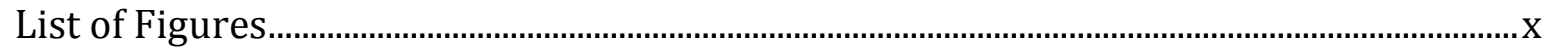

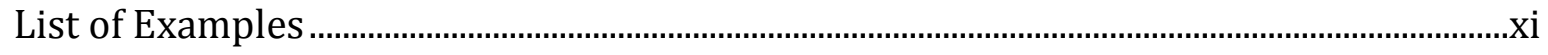

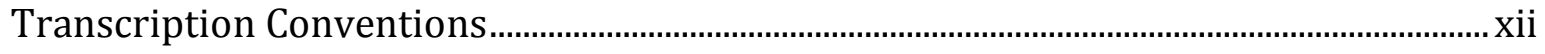

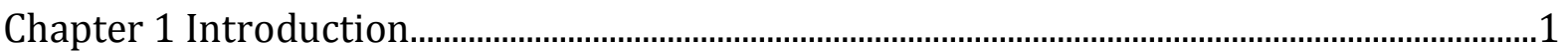

1.1 Why research language in sport?

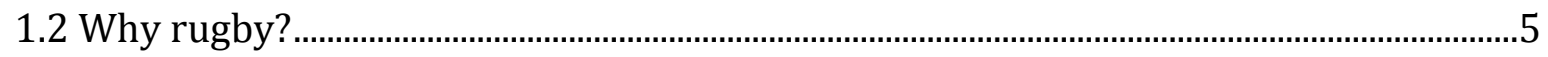

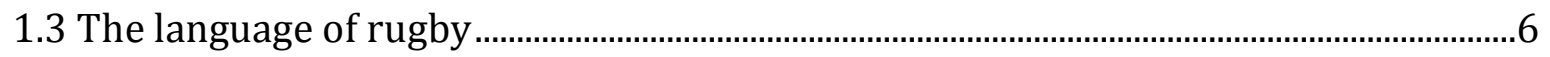

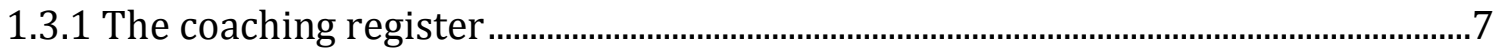

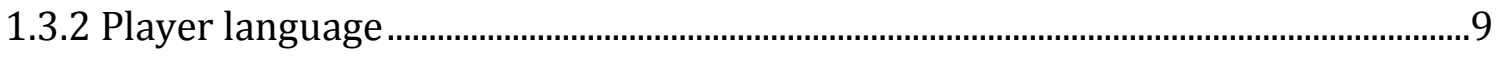

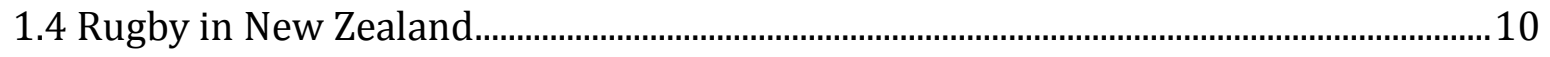

1.4.1 The place of club rugby in New Zealand ................................................................11

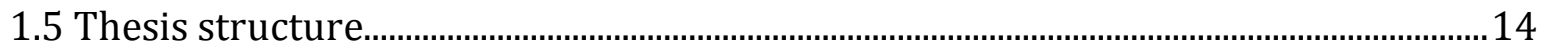

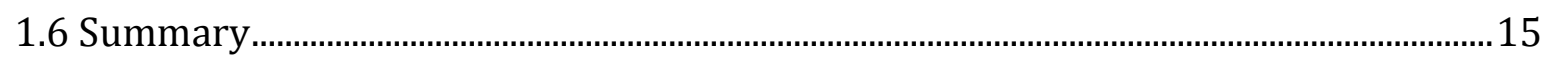

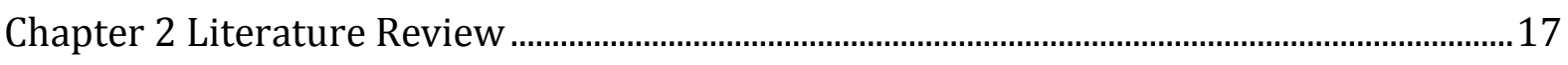

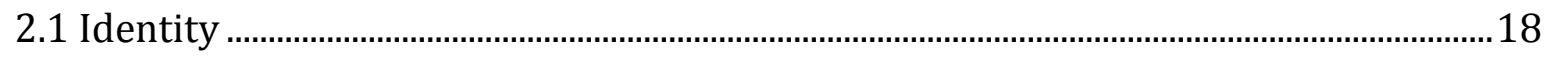

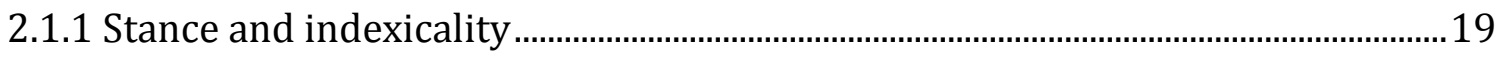

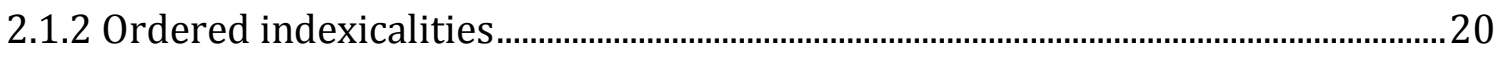

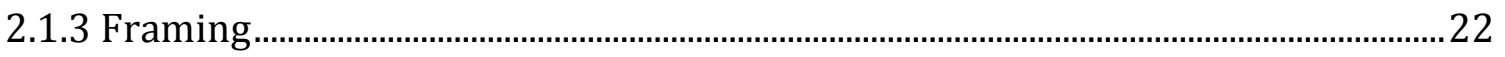

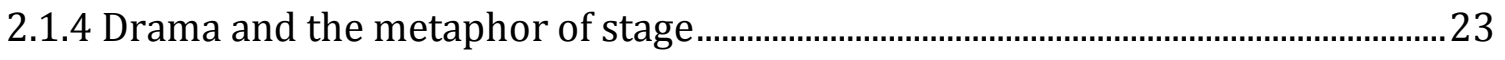

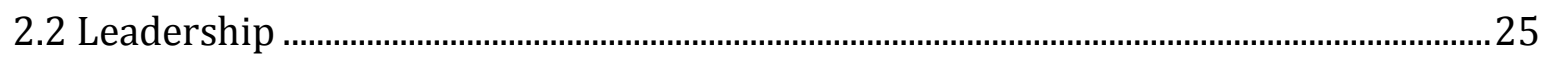

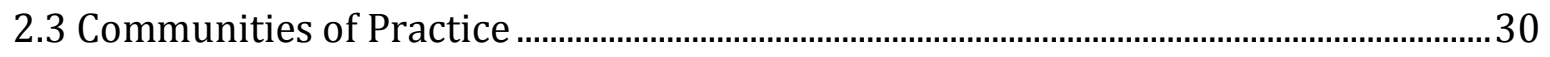

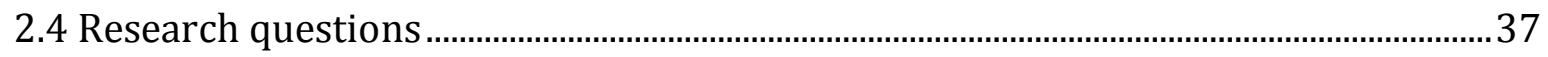

2.5 Interactional Sociolinguistics as a methodological framework ....................................... 37

2.6 Linguistic Anthropology and Linguistic Ethnography..................................................... 40

2.7 Summary 


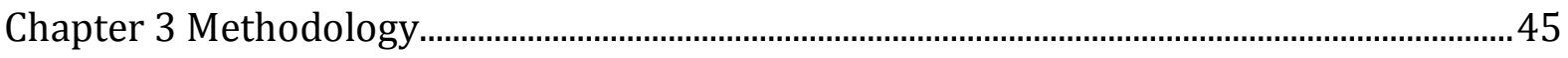

3.1 The Role of ethnography ............................................................................................................4

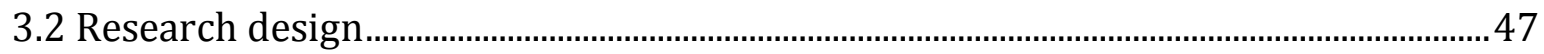

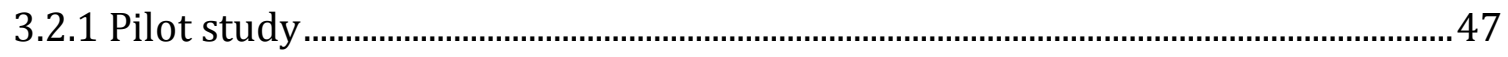

3.2.2 Constructing my role as an ethnographer......................................................................4

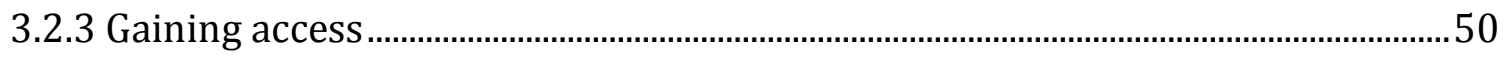

3.2.4 Initial observations and interviews ...........................................................................5

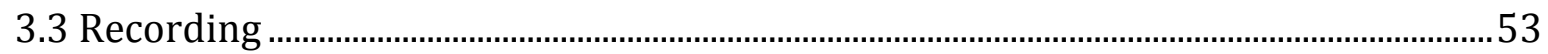

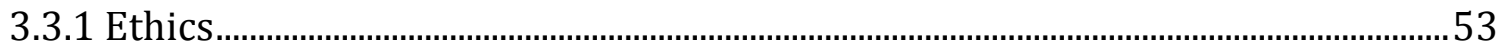

3.3.2 Recording equipment......................................................................................................5

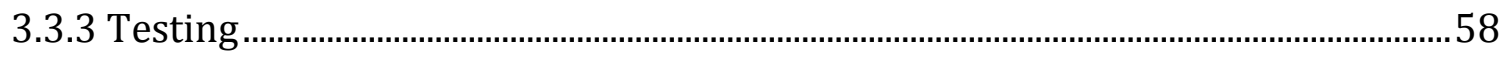

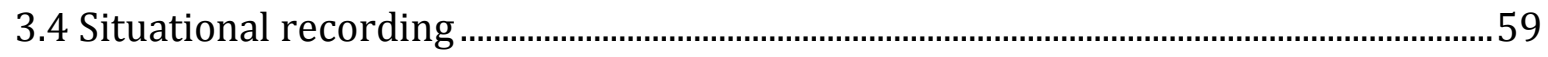

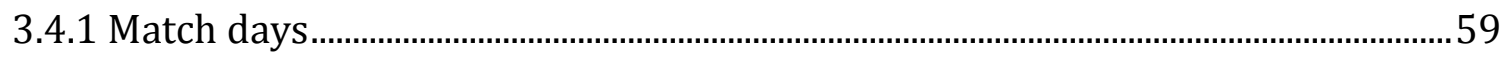

3.4.2 Training sessions.....................................................................................................................

3.5 Interviews, feedback and follow-ups ………......................................................................67

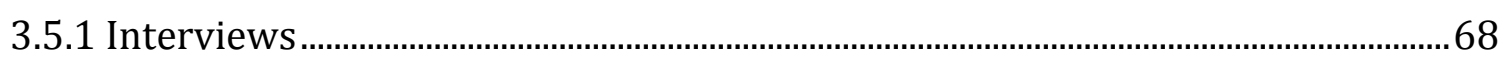

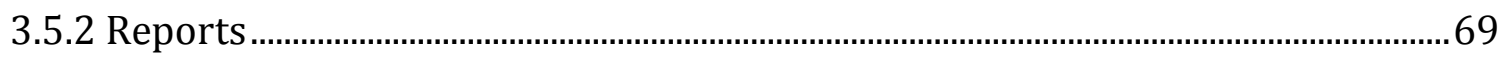

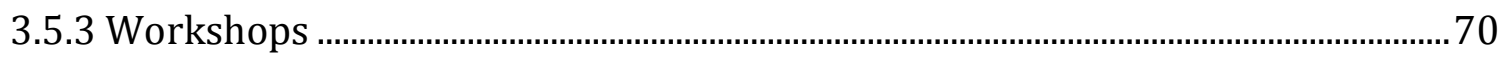

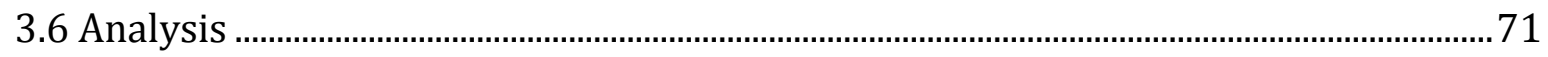

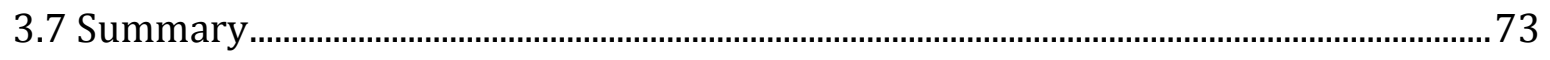

Chapter 4 Communities of Practice in the Rugby Club ...............................................................75

4.1 CofPs within CofPs ...................................................................................................................

4.1.1 Field position as the basis of CofPs...................................................................................

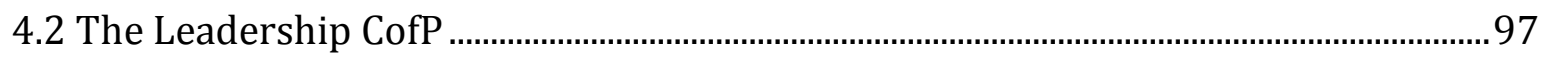

4.2.1 From plan to practice: The co-captaincy …………………………………………....97

4.2.2 Co-constructing leadership .................................................................................... 104

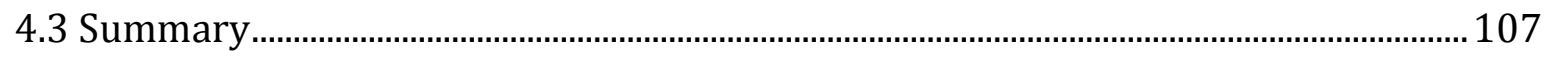


5.2 Constructing leadership back-stage

6.2 First order (direct) indexicalities

6.3 Second order indexicalities

6.4 Third order indexicalities.

6.5 Locally negotiated masculinities.

6.6 Other macro-identities.

6.7 Summary.

Chapter 7 Temporality: The renegotiation of leadership and identity.

7.1 Ritual and performance.

7.1.1 Pre-match ritual

7.2 Routine and formulae.

7.2.1 The team meeting

7.2.2 The pre-match huddle

7.2.3 Formulaic language.

7.3 Re-negotiating the routines of leadership ritual 185

7.4 The last rites of the team CofP. 190

7.4.1 The post-match huddle 190

7.4.2 The final post-match huddle. 191

7.5 Temporality. 196 
7.6 Performance, identity, leadership and time

Chapter 8 Conclusions.

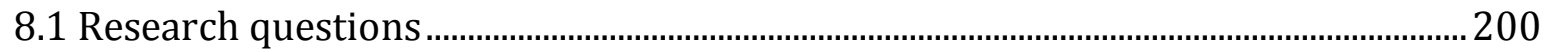

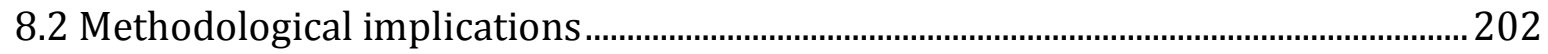

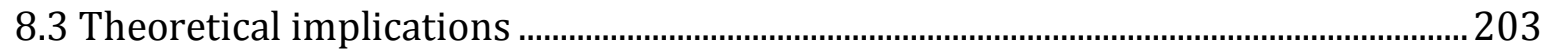

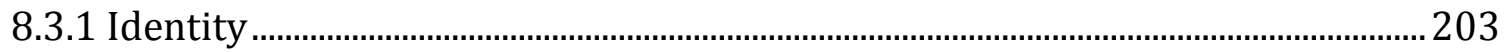

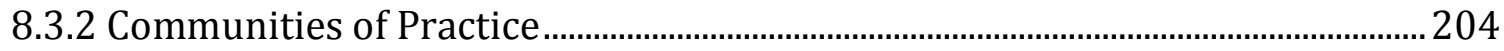

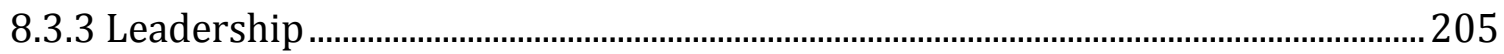

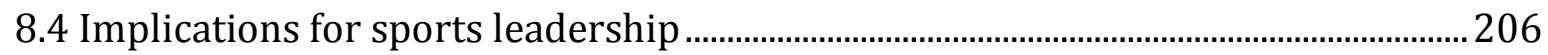

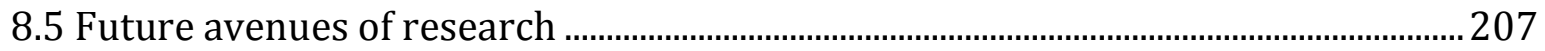

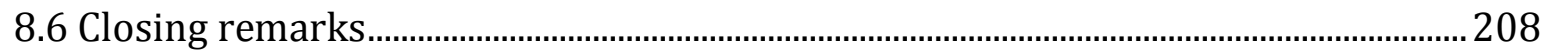

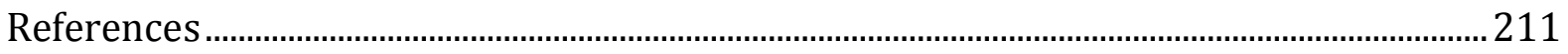

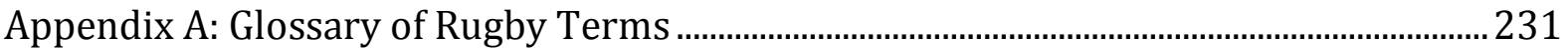

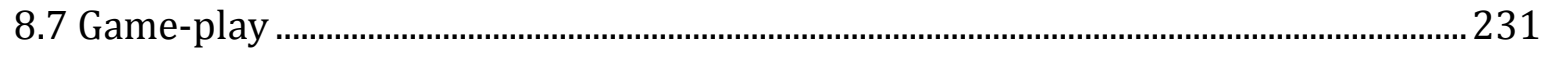

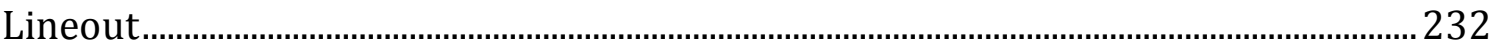

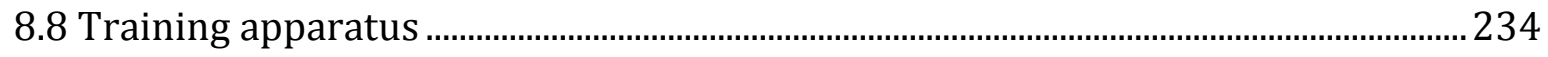

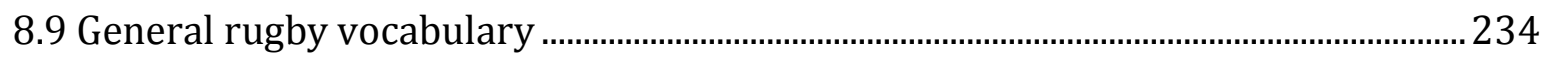

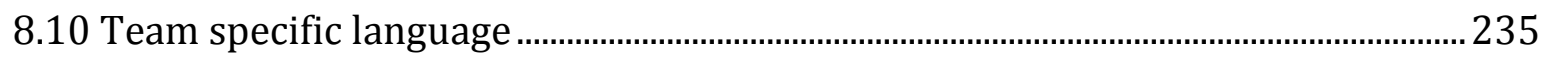

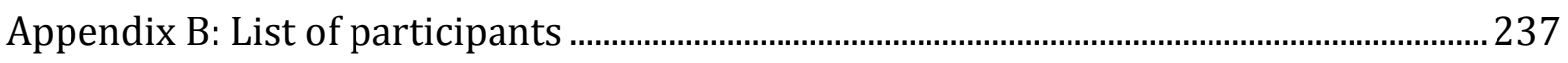

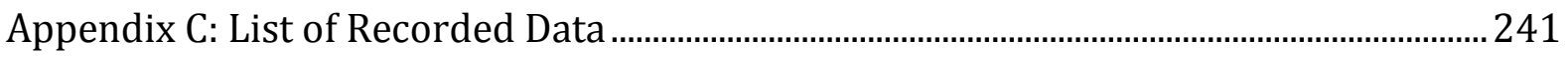

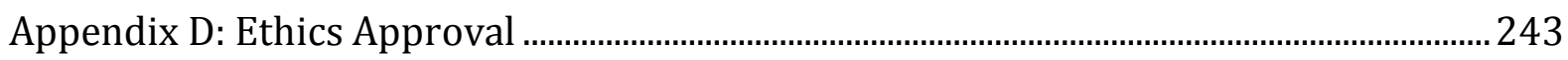

\section{List of Figures}

Figure 1.1: The four-tier structure of NZ (adult male) rugby with number of players.....12

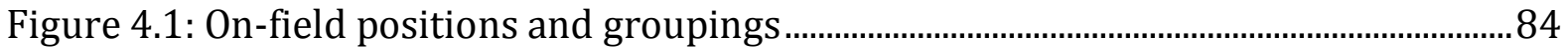

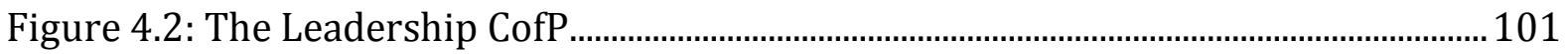

Figure 4.3: The Leadership CofP with trajectories ................................................................ 103 


\section{List of Examples}

Example 2.1: Interview with Mozza..........................................................................................27

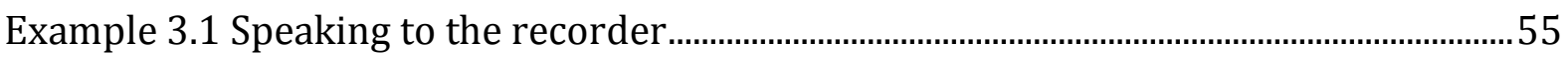

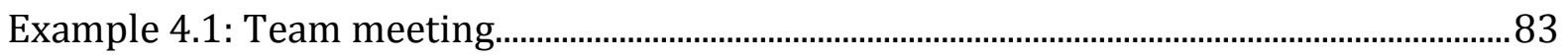

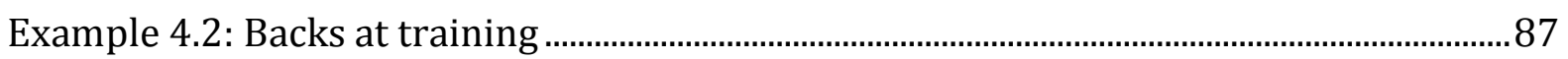

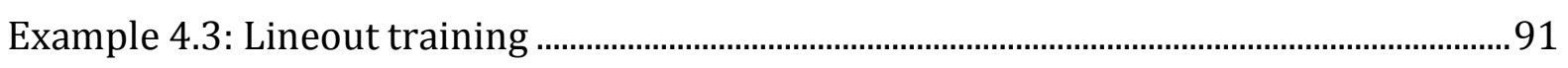

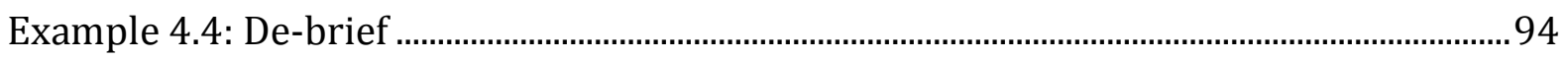

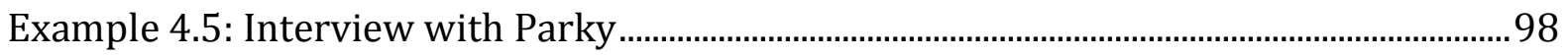

Example 4.6: Tommo and Jon discussing potential props .....................................................104

Example 5.1: Tommo and Jon discussing discipline ................................................................119

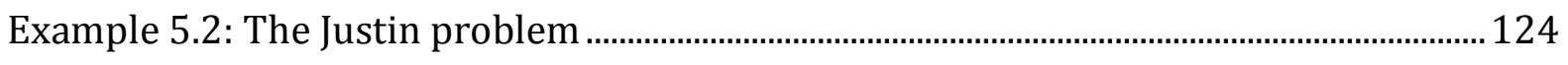

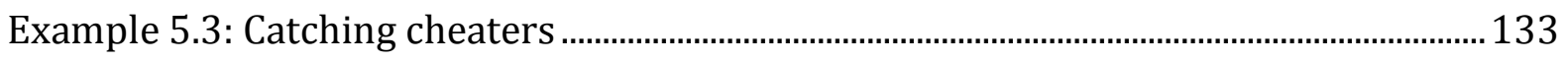

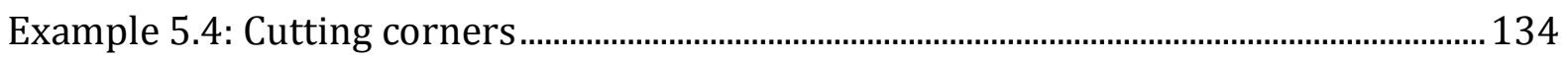

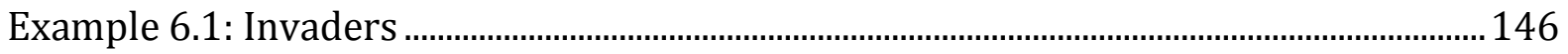

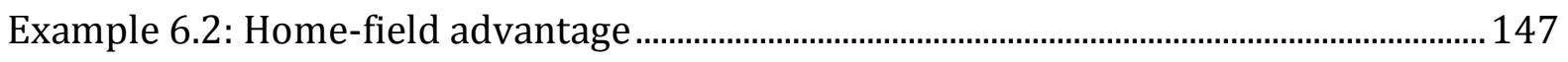

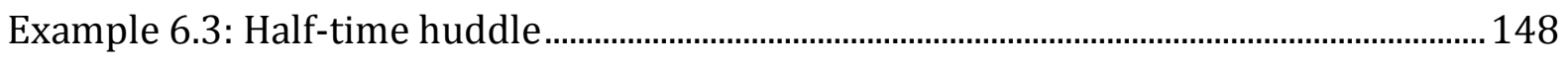

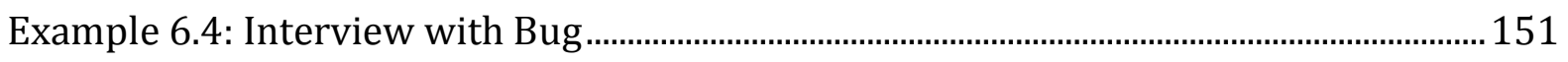

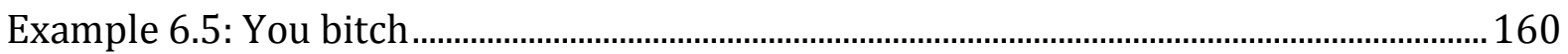

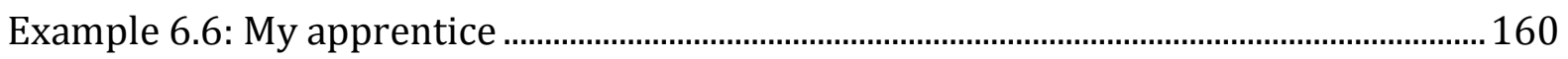

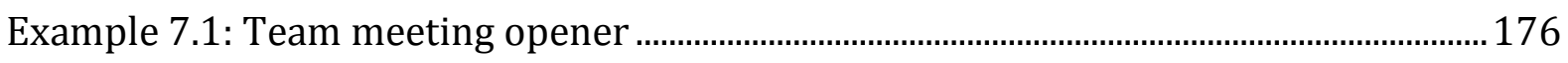

Example 7.2: Team Meeting............................................................................................... 179

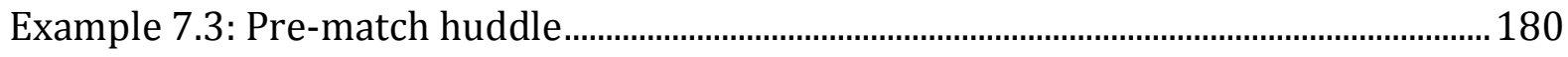

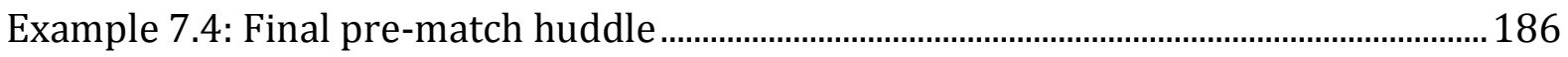

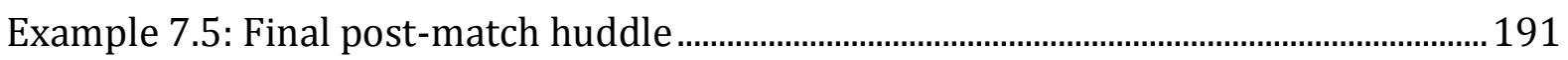




\section{Transcription Conventions}

Adapted from the transcription methods detailed by Celia Roberts at http://www.kcl.ac.uk/schools/sspp/education/research/projects/dataqual.html

\begin{tabular}{|c|c|}
\hline$/ /$ & final fall, indicating end of turn or emphasis \\
\hline / & slight fall, may end turn or suggest more to come \\
\hline$?$ & rising tone, indicating question \\
\hline , & slight rise to indicate continuation \\
\hline. & pause of half a second or less \\
\hline$\cdots$ & pause of between half and one second \\
\hline$\cdots$ & Untimed pause of longer than a second \\
\hline$<3>$ & timed pause \\
\hline$=1$ uh huh $1=$ & indicates overlap boundary, e.g.: \\
\hline \multirow[t]{3}{*}{ (number used to disambiguate overlaps) } & A: $I$ was $=1$ going $=2$ to the $1=\operatorname{shop} 2=$ \\
\hline & B: =1 uh huh $1=$ \\
\hline & C: $=2$ were you $? 2=$ \\
\hline$==$ & indicates latching onto previous utterance \\
\hline- & $\begin{array}{l}\text { Used for interruptions, self-corrections and false } \\
\text { starts. }\end{array}$ \\
\hline [coughs] & noise or action (e.g. [opens door]) \\
\hline \multirow[t]{3}{*}{$[$ laughing $]$ I don't know } & noise or action that overlays speech. Also used to \\
\hline & give extra information about speech e.g. $\{[$ louder $]$ \\
\hline & what ?\} \\
\hline
\end{tabular}




\section{Chapter 1 Introduction}

For 50 years the team's environment was coach-driven. Players didn't have to think for themselves. They were told what to do and when to do it. Not surprisingly, when they were on the field and forced to make decisions on their own, many players struggled.

[Graham] Henry decided to change the model and give the players unprecedented responsibility by creating an 11-man leadership group. He took the simple view that making strong decisions off the field would lead to better decisions being made on it. These senior players were empowered to construct their own parameters, shape the team culture and determine the codes and ethics by which they would live.

(Paul 2007)

The above quote is from a newspaper article discussing Graham Henry's successful approach to coaching the New Zealand national rugby team, the All Blacks. It illustrates the sea-change in the way that leadership is viewed in rugby. Historically, rugby leadership began and ended with the coach. However, this has changed considerably with the professionalization of rugby, with many teams now emphasising leadership as a team process. This thesis examines how this distributed model of leadership is enacted in language, and by using a grassroots team as the source of the data, shows that it has now reached non-professional teams. While this leadership model is also used in other organisations, the sports team can be viewed as a unique type of organisation because it deals with an activity that can be considered both work and play. Furthermore, sport itself represents a cultural artefact and much of the interaction that characterises sport is grounded in ritual. For these reasons, examined in greater detail below, sports teams represent a rich source of data for research on the intersection of leadership, culture, and identity. 
Leadership and communication are considered an important part of team sport, yet they are rarely skills that players are taught, the expectation being that they should acquire these skills in their non-sporting life (Paul 2007, 2008; Telfer and Ferguson 2006). However, in professional sport the lack of these skills is a growing point of criticism by coaches and sports media (e.g. BBC Sport 2009; Paul 2008). For these reasons, it is worth considering how sports players (and coaches) can be given the tools to better present themselves as leaders. This thesis takes the view that a key way to do this is through facilitating their self-analysis of the way they use language in enacting leadership. Although there have been very few studies of leadership discourse in sport, leadership communication is a topic which has been extensively studied by linguists in workplaces. One of the goals of this research is to compare the findings from research on workplace leadership practice to the discursive practices found in a rugby team.

Like other social constructionist approaches to leadership, this research locates leadership within a Community of Practice (CofP). Inevitably, this also involves identity, specifically group identity, in the analysis. Thus the linguistic construction of leadership is not examined in isolation but in a reciprocal relationship with how a CofP is formed and the group identity that is created as a result. This is further enhanced through the linkage of local practices to global ideologies that exist within rugby and sporting culture.

A further aim of this research is to build upon the existing research carried out on leadership discourse and leader emergence through the application of a CofP approach. However it is also intended to exemplify the richness of sport as a research site for sociolinguistics. To this end, this research provides an ethnography of a New Zealand rugby team, with authentic recorded interactions used to analyse the way in which players and coaches construct themselves as leaders and show how a coherent group identity is constructed. For this purpose an ethnographically-led form of discourse analysis, Interactional Sociolinguistics, is used.

The fieldwork which informs this research is an ethnographic study of the highest level senior team in an amateur rugby club in New Zealand. A year was spent working closely with the team at training sessions and on match days, allowing the collection of some 


\section{Introduction}

thirty-two hours of recorded interactions ${ }^{1}$. These were later analysed to reveal the communicative practices within the team concerned with leadership and the construction of team identity. The ethnographic observations also yielded a detailed understanding of the social and organisation structure of the team, which was used in analysing the linguistic data that had been collected. Further detail and the importance of this ethnographic data collection and analysis process are discussed in Chapter 3.

As Chapter 2 focuses on the theoretical and methodological frameworks used in this research, this chapter will first present a case for doing linguistic research on sport. It then provides some background on the language of rugby and the way in which rugby is structured in New Zealand. It should be borne in mind that as a non-New Zealander my acquisition of the latter should be considered a part of the ethnographic process that this research represents, while it has been knowledge of the former that facilitated my integration into the research site and was the catalyst for my interest in rugby as a site for sociolinguistic research.

\subsection{Why research language in sport?}

Starting with a broad view of sport in general, and narrowing to team sports in particular, there are three main reasons for researching the language of sport which are pertinent to research presented here.

Firstly, there is the anthropological view of sport; as a cultural artefact (Kuiper 2010, personal communication). Language encodes culture, and as such an examination of the different ways in which sport is 'done' through language can show how the culture of sport is interpreted and renegotiated by the participants in the sport (Meân and Halone 2010). This view can be applied to a range of linguistic domains. For instance, why research academic language? Why research media language? The anthropological view is that by being present in societies these linguistic domains should be researched because the way in which people use language to participate in these domains is dependent on the cultural norms that surround these domains and this is turn can inform a wider socio-cultural analysis of a society. It follows that researching the language of sport is research on the creation of a culture that has meaning in the lives all

\footnotetext{
${ }^{1} \mathrm{~A}$ full list of the recordings and the exact total duration of each recording is given in Appendix $\mathrm{C}$.
} 
people who participate in sport. Furthermore, the wide use of sporting idiom in many languages and the use of sport as a referent in daily interactions highlight its cultural pervasiveness. Thus we have the first reason why sport should be of interest to sociolinguists. If nothing else, it is at least as valid to research sporting language as it is to research academic language or media language as a particular domain or genre of language.

Secondly, there is the sociological (critical) view of sport as an institution that has the perpetuation of hegemonic masculinity as its raison d'être (Anderson 2009; Connell 2005; Nauright 1996). Sports sociologists suggest that sport was created in order to preserve masculinity from "going soft" in the face of a society in which men were predominantly raised by women (Anderson 2009:25). While this point is highly contentious, researching the language of sport can thus show how participants discursively construct their gender identity in the face of the hegemonic nature of sport. Although this thesis will deal with this very complex topic only briefly, it is important to acknowledge that it provides a social context in which sport is defined by many, especially those who do not engage with sport and are highly critical of its value to society. Furthermore, because sports sociology literature is replete with analyses of the many masculinities that are constructed in relation to sport (e.g. Adams, Anderson and McCormack 2010; Clayton and Humberstone 2006; Dunning 1986; MacLean 1999; Messner 1995; Nauright and Chandler 1996; Phillips 1996), this thesis treats masculinities as ideological reference points that are used in the dynamic construction of an individual's identity through stance. I do not suggest that masculinity is a fixed concept. Indeed the very notion of masculinity is a highly individual one constructed differently by every man (and women (Halberstam 1998)). Any individual is likely to construct their masculinity differently in different circumstances and for different purposes, just as they would any other aspect of their identity.

Thirdly, from the point of view of leadership studies, researching the language of team sport is useful as a sports team is a small hierarchical organisation with multiple leadership roles. Thus within a sports team one can easily analyse not just one leader, but several and the examination of the interplay between their leadership styles can inform a sociolinguistic analysis of how co-leadership can be effectively performed in small groups. Furthermore, due to the temporally situated nature of a sports team (in 


\section{Introduction}

that it is bound by the cycle of sporting seasons), sports teams provide a site in which leadership emergence can be studied. As this thesis will show, this is also bound up with the negotiation of communicative practice and the construction of group identity.

These three reasons for researching sport can all be addressed through a focus on language. This thesis draws upon all three, but it is the third of these, leadership, in conjunction with identity which provide a central focus in this research.

\subsection{Why rugby?}

To return to the anthropological point explained above, if a sport is viewed as a culture (or sub-culture) the players and coaches can be considered the "natives" of the sport. In engaging with sport, players and coaches learn the recognised cultural behaviours, or practices, with which they are identified as being a part of the sporting culture (Anderson 2007; Birrell 1981; Donnelly and Young 1985; Hargreaves 1982). Rugby players and coaches have a shared cultural knowledge about rugby and the way that they interact within the bounds of the rugby club is not just a way in which they reproduce rugby culture, but how they construct themselves as rugby players using the socio-cultural resource available to them as natives of the sport. The reason that rugby was chosen rather than another sport is that I am a native of the sport. Therefore I already carry the socio-cultural knowledge of how to 'do' rugby and am able to interpret the interactions that take place within the team with greater ease than a non-native. In saying this, I am a native of rugby, but not of New Zealand. I am thus both insider and outsider, a perfect position for an ethnographer as it eases access and acceptance but allows for objectivity to be retained (Hammersley and Atkinson 1983).

Focusing on New Zealand, rugby ${ }^{2}$ is held up by many as symbolic of New Zealand national identity (Crawford 1985; Falcous 2007; Grainger 2009; MacLean 1999; Nauright 1990; Phillips 1996). While this representation may be contested by individuals with no personal involvement with the sport or by those actively opposed to it (MacLean 2000, 2003; Thompson 1988), it is undeniable that rugby plays an influential part in New Zealand society through its connections with media, business

\footnotetext{
2 In New Zealand, unlike England or Australia, "rugby" is always taken to mean rugby union, not rugby league, which is referred to as "league". As this thesis concerns rugby in New Zealand, the New Zealand practice is adopted, although it should be noted that this is also the practice in Scotland, Ireland and Wales. A full glossary of global and local rugby terminology can be found in Appendix A.
} 
and education, not to mention the role played by rugby in the nostalgic view of New Zealand history (Nauright 1996; Ryan 2005a). While this thesis does not look at rugby overly critically, it is acknowledged that rugby, and sport in general, plays a part in the perpetuation of masculine hegemony (Adams et al. 2010; Bryson 1987; Nauright 1996; Tagg 2008; Thompson 1988) and in post-colonial subjugation of culture (MacLean 1999, 2003; Melnick and Thomson 1996; Obel 2007; Palmer 2000; Schaaf 2006; Watson 2007). This is incorporated into the analysis of how rugby ideology is indexed in interaction, presented in Chapter 6.

Another view that may be taken of a rugby team is to view it as an organisation that resembles a workplace. This approach, as well as the cultural view of sport, has been taken in this research and the organisation of the rugby team is analysed in ways consistent with research on workplace language. Furthermore, the rugby team is an organisation concerned with the improvement of individual and team performance. Thus it is unsurprising that many successful top-level rugby coaches also have extensive experience of teaching (e.g. Graham Henry, Jim Telfer), suggesting a link between rugby coaching language and classroom language. As the rugby team can be viewed as a learning environment, it is highly suitable to analyse its organisational structure through the lens of Communities of Practice (Lave and Wenger 1991; Wenger 1998a), a theory of situated learning which has been widely taken up in sociolinguistics (Eckert and Wenger 2005; Holmes and Meyerhoff 1999).

While team performance is ultimately measured in match results, there are a large number of other indicators used by coaches to measure player performance and one of these performance factors is communication. Players that are viewed by the coaches as good communicators are often seen as leadership candidates (Sullivan and Feltz 2003; Zhang and Jensen 1997). In other words, by displaying the discursive practice that coaches view as leadership practice, players position themselves as potential leaders. This means that by analysing the communicative practices of players and coaches, the emergence of player leaders in a team can be analysed.

\subsection{The language of rugby}

While, as noted above, rugby can viewed as an artefact of culture, it can also be viewed as a global sub-culture in itself, with a history and language of its own. Membership of 


\section{Introduction}

this culture is open to any person who plays, watches or simply has an interest in the game of rugby, with varying levels of integration. This is displayed in the use of rugbyspecific language. At the most obvious level this is the use of rugby jargon related to game-play by players, coaches and fans, such as ruck, maul, scrum, and line-out. In using this language individuals can construct an identity as someone conversant with the socio-cultural knowledge that underpins rugby culture, enabling them to position themselves as a player, a coach or a rugby fan. At another level there is the use of formulaic language in rugby media broadcasts, which is also a part of the genre of sportbroadcasting. This involves accepted practices regarding the way in which commentaries are performed (Kuiper 1996), the structure of post-match interviews (Emmison 1987, 1988; File forthcoming), and feeds back into the way in which coaches and players talk, sometimes indexing these styles in the "event-casts" (Heath and Langman 1994) used to describe training exercises. However, my research is concerned with language that is used within the team, not the external representation of the team in the discourse of on-lookers. Therefore, it is the discourse of the coaches and players that is the focus. The following section details the existing research carried out on the language of coaches and players. This serves to underline how little research has so far been carried out on this topic and contextualises the current research within a wider research space.

\subsubsection{The coaching register}

The register of sports coaching appears to be situated somewhere between an academic register and a workplace management register (Heath \& Langman 1994; Masterson, Davies, \& Masterson 2006). However, depending on the team, the rugby coaching register could be positioned at any point along this continuum, with school rugby teams, most commonly coached by teachers, at one end and professional teams with professional coaches and a more business-led organisational model at the other. It is ironic then - or perhaps inevitable, that many professional coaches come from a teaching background; the most prominent example being the current All Blacks Head Coach, Graham Henry. In addition, the distinction between coaches and teachers has been remarked upon by Jim Telfer, then Director of Rugby for the Scottish Rugby Union, who publicly suggested in 2002 that all coaches in Scotland who worked with players in "their formative years" be called rugby teachers and those responsible for older players 
to be called rugby coaches (Telfer and Ferguson 2006). The idea behind this was that "a teacher looks at development, a coach looks at performance" (2006:133).

While Heath and Langman (1994) acknowledge that the register of rugby coaching originates from academic registers, they argue that it serves purely as the starting point from which it has evolved into a different, more specialised register. The main reason for this appears to be the physicality of sport and the fact that "action scripts the talk" (Heath and Langman 1994:85). Looking beyond this, Heath and Langman identify two discourse level features of coaching talk that distinguish it from other registers. Firstly, there are "philosophical setups" (1994:87), which serve to instantiate the rules of expected behaviour within the team as the coach sees them. The other type of coaching discourse is labelled an "eventcast" which includes the explanation of and instruction during training drills as well as addresses made by the coach to the team that are concerned with matches such as team huddles and pre-match team talks.

Both of these features can be found in the discourse of the rugby team studied here. But it is not simply the way in which coaching (and player) discourse is constructed that is the focus of this research. It is also the link that these have with the creation of identities within the team. In creating a coaching identity, coaches adopt styles of coaching as a form of leadership. While within the field of sports science there is a wealth of literature regarding sports coaching, mostly in the form of manuals on how best to approach it (Gourley 2003; NZRU 2007a; Turman 2003), these almost invariably take the position that the coach determines the style of leadership that is used. This is not the view taken in this research, nor does it appear to reflect the current practice in many teams, as the quotation at the start of this chapter illustrates. As is explained further in the theoretical overview provided in Chapter 2, leadership is seen as a reciprocal process in which an effective leadership style and strategy are determined through the interaction of leader and followers and that a multiplicity of leadership identities are constructed in the process of enacting effective leadership.

However, as outlined in the opening of this chapter, the current sports philosophy on coaching suggests that modern coaching should follow a more inclusive model than was traditionally adopted, taking into account player input and being more adaptable to situational changes. This shows that there is some common ground between 


\section{Introduction}

approaches to leadership in sports and the study of leadership in other fields. For instance, Gourley (2003) suggests that modern rugby coaching is about more than just leading players through practices and motivation. Along with others working in the field of sports psychology (Copeland and Wida 1996; Gordon 2007) and sport management (Auld and Cuskelly 2006; Gilbertson, Blyde, Gianotti, Gilbertson, et al. 2006), Gourley makes the point that the day of the hard-line authoritarian coach is over; there is now a requirement for coaches to be more supportive of their players.

While the research presented here focuses in part on the coaches in the team and the way in which they enact leadership, it also examines how leaders emerge from amongst the players and how they construct themselves as a team. As this thesis demonstrates, coaches construct the initial leadership practice with which other members in the team align in becoming leaders, and thus it is important to examine both coach and player language in studying leadership discourse in the rugby team. To the best of my knowledge, this is the first study that examines player language in this way, although it has been examined in relation to other research topics, in particular the construction of gender identity (Anderson and McGuire 2010; Donnelly and Young 1985; Kuiper 1991; Schacht 1996).

\subsubsection{Player language}

Although there has been very little research into the language of rugby players, the language of other sports players has been researched, specifically amateur soccer players (Clayton and Humberstone 2006; Corder 2004; Harris 2007; Meân 2001). As with rugby, these studies have mostly examined the construction of gendered identities, both of women's participation in what is conceived of as a "man's game" (Ferguson 2004) and of men as an analysis of what constitutes masculinity. It is the latter that is of most relevance to this research and yet, although there is a great deal of sociological literature about gender in sport, there is very little in the way of sociolinguistic research concerning sports participants. Although there is a sub-field of sociology that focuses solely on sport, sociolinguistics has yet to capitalise on this large resource of potential research subjects. In the linguistic research that has been carried out on male sports teams, and hence the most relevant to this research, there have been some detailed analysis of locker room talk (Clayton and Humberstone 2006; Kuiper 1991), which in most cases includes the trading of insults between the players. This is not just confined 


\section{N. A. Wilson}

to sports teams, it is also noted in research on other all-male groups (Boxer 2002; Cameron 1997; Kiesling 2001a, 2001b), highlighting the relevance of this research in the study of masculinity.

However, the focus on player language in this research is on the emergence of leaders from among the players, who position themselves as leadership candidates through their interactions with other players and with coaches. This is a topic that has not been researched from a linguistic standpoint, although Robin McConnell $(1996,1998)$ carried out detailed ethnographic research on leadership amongst the All Blacks prior to the professionalization of rugby. McConnell's research focused on the structure of events within the All Blacks, but did not look at the interactions of the players in detail, taking a more macro, sociological view of the team as a social grouping. This research therefore, is the only research that takes a detailed view of the way in which language is used by leaders in a rugby team.

The following section establishes the organisation of rugby in New Zealand, thus outlining the socio-cultural background against which a New Zealand club rugby team is measured and providing information that lends depth to the description of the rugby team described in this thesis.

\subsection{Rugby in New Zealand}

Interactional Sociolinguistics regards context as being crucially important for the analysis of interaction and, using ethnography, the immediate context of the team's behaviour will be thoroughly described. However, this too must be contextualised against the wider backdrop of New Zealand rugby. Much has been written on the history of New Zealand rugby from the first club match which took place in Nelson on $14^{\text {th }}$ May 1870 (Chandler 1996; Nauright 1990) to the first touring All Blacks side (Ryan 1993), through to the controversy surrounding matches against South Africa during apartheid (MacLean 2000; Nauright 1990). While these are all important factors in the development of rugby culture in New Zealand and have helped secure a place for rugby in New Zealand's national identity, it is the move from amateur to professional sport which has had the greatest impact on the structure of the modern game and the way in which the players and coaches operate within it (Martin 2008). The organisational structure of the leagues and competitions is regularly changed and the NZRU is often 
the target for criticism regarding the way they oversee the national game (Obel 2001; Owen and Weatherston 2002). This section summarises the structure of rugby in New Zealand. This is important for this research as the team focused on is a Premier grade team, which means that it is the highest level of amateur rugby in New Zealand, with the next step for skilled players to gain a (semi) professional contract with a provincial representative side.

In keeping with the ethnographic approach taken in this research and the focus on amateur rugby, the structure of New Zealand rugby will be described from the grassroots up, rather than from the elite level down (c.f. Obel 2001; Owen and Weatherston 2002). This highlights the importance of amateur rugby to New Zealand rugby culture and views the potential career advancement of a highly-skilled player through the different levels of the New Zealand system. This approach demonstrates clearly how the team that took part in this research is a part of a larger system and that the players involved move within this system. Indeed, since the completion of this research, several of the players concerned have advanced to professional rugby.

\subsubsection{The place of club rugby in New Zealand}

Club rugby, the grassroots as it is often labelled, is where rugby culture in New Zealand traditionally resides. The NZRU states that "community rugby is integral to rugby in New Zealand" and as such funded it to the tune of \$27.6 million in 2009 (NZRU 2009). While for many children their first experience of rugby is at school, it is the rugby club that is the heart of community rugby and the site of the majority of work by the NZRU via the provincial unions to promote rugby and encourage wider participation. A rugby club is made up of many teams, from children's teams up to premier teams, the exact structure of which will be detailed in the course of describing the team that took part in this research. It is a premier team which is the focus of this research and this section serves to explain why this type of team is of interest; it lies on the edge of community rugby, with the next step for ambitious players being provincial rugby and a prospect of professionalism. It should be pointed out that in this section, unless otherwise stated, it is men's rugby that is described. This is simply because the focus of this research is a men's team and the vast majority of literature (about organisational structure of rugby) deals with men's rugby. 
As a result of the move to official professionalism in 1995 (FitzSimons 1996; Obel 2001; Owen and Weatherston 2002), amateur rugby has taken somewhat of a back seat in recent times. However, there is regular acknowledgement of the grassroots of the game both by the rugby press (NZ Herald 2010) and by the governing unions (NZRU 2007b, 2009). Club rugby is the only part of the four tier structure where rugby can be regarded as truly amateur, with the players "playing for the jersey". The layers in this structure are embedded, as shown in Figure 1, so that a player moving on to the next level of rugby retains his membership of the teams from previous levels. As can be seen, the vast majority of players (88.4\%) only ever play club rugby. Although there are 520 rugby clubs in New Zealand, only 364 of them field premier grade teams (Zinn 2004:88), giving 9464 as an estimate of the total number of premier grade players in New Zealand (assuming an average squad size of twenty-six).

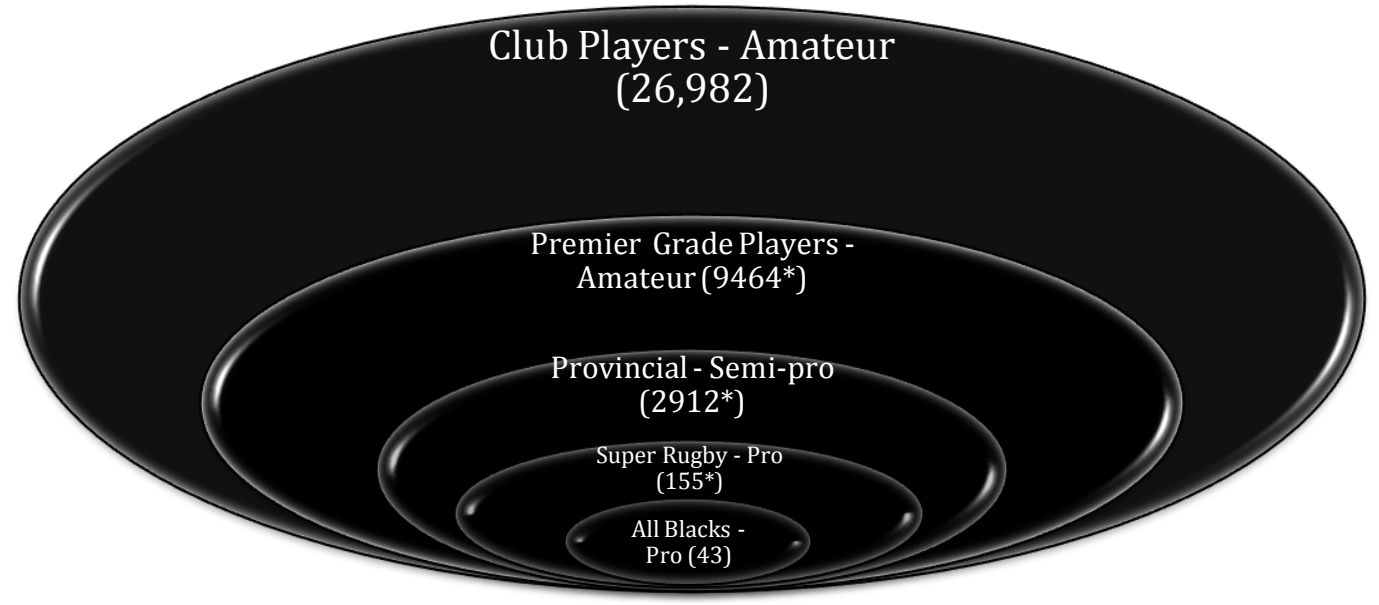

Figure 1.1: The four-tier structure of NZ (adult male) rugby with number of players

(*estimate based on average squad size)

All senior rugby players in New Zealand are affiliated with a club. They may have played for that club since they were boys or they may have switched clubs several times, perhaps as a result of geographical mobility. The club that a player plays for then determines the province for which he can be selected. For instance, a player playing for a premier team in a club within the Wellington region could be selected for one of the Wellington representative sides, of which there are several levels: Colts (under 21), Development, Māori, and the Vodafone Lions. The first three teams are semi- 


\section{Introduction}

professional in the sense that the players are paid expenses or loss of earnings but are not actually paid a wage to play rugby. The Vodafone Lions are the province's team that competes in the National Provincial Championship ${ }^{3}$ and the players within it are fully professional.

A player that is successful in being selected for one of the semi-professional representative sides may go on to play for the Lions, thus becoming a professional rugby player on a contract with the Wellington Rugby Union. If the player does well with the Lions, it is possible that he will be signed by one of the Super Rugby franchises. This puts him on a contract with the NZRU who also buy him out of his provincial contract. He may at some point then be selected to play for the All Blacks, the highest point of achievement for a New Zealand rugby player. However, at every stage, the player retains his club affiliation (as long as he remains within that province) and is obliged to play for the club when not engaged on other rugby commitments. In reality this means that an All Black might play only one match for his club in a season, but a player who plays for an NPC team may well play the first half of the club season in amateur rugby. If a player who makes it as far as an NPC team like the Wellington Lions does not progress any further up the professional rugby ladder, he finds himself in a grey area where he is not really a full-time professional rugby player as he only plays professionally for the duration of the provincial season. The NZRU would not define such a player as a professional rugby player, hence Provincial rugby should be considered semi-professional:

It is the view of the NZRU that is it only when a player is selected (or potentially in the future contracted to) a Super Rugby team that he can truly be considered a professional rugby player in New Zealand. (NZRU 2008:25)

This means that there exists within New Zealand rugby a multi-layered structure of professionalism and a clear progression which a rugby player may follow in pursuing rugby as a career. Taking the NZRU's definition further, which implies that there is a degree of ambiguity as to the professional status of NPC players, the premier grade of

\footnotetext{
3 Currently known as the ITM Cup, but the sponsor and format changes regularly and most rugby supporters continue to use the name used for many years: the NPC.
} 
club rugby is the highest level at which a player can truly be considered to be an amateur.

The premier team within a club is generally composed of the players who have the greatest skill level and desire to play rugby at the highest level they can. For many of these players club rugby is the only level of rugby that they will ever play, others may be selected for their provincial side, and some will become true professional rugby players, playing for a Super Rugby franchise and maybe the All Blacks. Thus the amateur rugby club has a dual role to play in New Zealand rugby. It is where rugby tradition is strongest, and there is a sense of continuity as past-players work as committee members, coaches, ground staff etc. (NZ Herald 2010). Yet the club is also where any player embarking on a professional rugby career must start. Every professional player in New Zealand is affiliated with a club, usually the same club that they have played for since their teenage years (or younger), as demonstrated in Figure 1.1. This provides a link between professional and amateur rugby that fosters ambition among the amateur players and serves to (perhaps) remind the professionals of their rugby background.

The detail given here on the structure of New Zealand rugby is intended to provide the context in which the ambitions of the players in this study may be understood. It positions the players in the Premier team as standing on the cusp of professional rugby, and many see this as a realistic next step for them. In addition, during my time with the team, several professional players returned to the team, either to play due to nonselection for their professional team, or simply to visit their club and give support to the players. Thus the link between the levels of rugby in New Zealand is one that I have witnessed firsthand and the sense of community among rugby "natives" is strong, a fact which helped in both my acceptance by the team and in later analysis of their recorded speech.

\subsection{Thesis structure}

Chapter 2 represents the literature review of this thesis, examining existing literature on the topics of identity, leadership and the Communities of Practice model. It also presents the research questions to be addressed and introduces the methodological frameworks that have been used. These frameworks are further described in Chapter 3, which details the ethnographic development of the methods used to gain access and 


\section{Introduction}

acceptance in the rugby team and collect authentic interactions, and how decisions were taken regarding the selection of data. Chapter 4 is the first of three analysis chapters. It also provides details of the team organisation built upon in subsequent chapters by showing how the rugby team can be viewed as a collection of overlapping communities of practice. Chapter 5 expands upon the idea of leadership as a communicative social practice taking it from a negotiated practice between leaders and followers to a performance of team identity, a topic which is then taken up in Chapter 6. It looks at the complex topic of identity, focusing on how the team combines rugby ideology and local social practice to create a unique identity that furthers their goals as a team. Chapter 7 aims to unify the preceding three chapters through the theme of time, looking at formulaic language, repetition and ritual, as well as their relation to leadership and team identity. Finally, Chapter 8 summarises the conclusions that can be drawn from this research and potential avenues for future research.

\subsection{Summary}

This chapter has outlined the motivation for this research, my suitability as a researcher for this topic and the reasons why rugby is a legitimate area for linguistic research. It might be added that not only is sport a fruitful area for linguistic research, but that in other disciplines, notably psychology and sociology, there is an extensive tradition of research on sport. Given the increasingly interdisciplinary nature of the social sciences and humanities, linguistic research on sport is well positioned to both benefit from and contribute to the existing inter-disciplinary research on sport.

As specified in the opening segment of this introduction, the following chapter outlines the theoretical and methodological frameworks espoused by this research, as well as detailing the research questions that it addresses. 


\section{Chapter 2 Literature Review}

As the introductory chapter of this thesis has discussed, two functions of language as used in a New Zealand rugby team provide the foci of this research: the enactment of leadership and the creation of team identity. These are closely related as both are concerned with the discursive construction of identity. This chapter details the theoretical stance I take on identity and leadership with reference to existing research and theory. These two concepts are seen as linked because leadership can also be viewed as an aspect of identity. However, leadership can also be seen as a social practice, and explained with recourse to the Communities of Practice model (Wenger 1998a). This in turn incorporates the construction of identity as it relates to group membership. Each of these conceptual frameworks is linked to the other two in some way, and hence there is some degree of crossover in terms of the literature that is pertinent to each. Theories of linguistic identity construction and collaborative social practice are thus drawn upon in examining how leaders in a rugby team "do" leadership. These theoretical approaches are united in my chosen methodology of Interactional Sociolinguistics, in which ethnography plays an important role.

This chapter is therefore organised as follows. Section 2.1 opens the discussion on the linguistic construction of identity, and is structured around Bucholtz and Hall's (2005) principles of identity and interaction, which are influential in the analyses presented in this thesis. Section 2.2 introduces the existing linguistic research on leadership, starting with leadership as an identity and moving on by way of leadership styles and stereotypes to leadership as a form of social practice. This provides the starting point for Section 2.3 in which the communities of practice model is detailed as it relates to language, identity, and leadership. It is in this section that detail is given of the rugby team that is the focus of this research. From this point on the chapter changes focus from theoretical background to the application of theory through research methodology. In order to direct this, Section 2.4 outlines the research questions that are addressed in this thesis. Sections 2.5 and 2.6 discuss Interactional Sociolinguistics as a methodological framework, showing how this approach can be used to carry out 


\section{N. A. Wilson}

research on leadership and identity, and emphasises the importance of ethnography to this research and to the analysis of interaction in general.

\subsection{Identity}

Identity is a relational and sociocultural phenomenon that emerges and circulates in local discourse contexts of interaction rather than as a stable structure located primarily in the individual psyche or in fixed social categories. (Bucholtz and Hall 2005:585-6)

Following Bucholtz and Hall I treat identity as a socio-cultural phenomenon that is created through language, but which also influences language. Identity is viewed not as being internal to a person but a resultant product of interaction. However, once created, identities can be further referenced in later discourse; thus identity and discourse can be viewed as existing in a circular relationship.

A good starting point for the discussion of identity as it is currently viewed in the majority of contemporary sociolinguistic research is Bucholtz and Hall's (2005) framework of identity and interaction. This specifies five principles of identity: emergence, positionality, indexicality, relationality, and partialness, which are discussed below in relation to other important sociolinguistic work on identity construction. As Bucholtz and Hall note in later work (2008b), their framework for socio-cultural ${ }^{4}$ linguistic research on identity has been widely taken up, clearly reflecting its applicability to a wide range of approaches to the study of language in society. Bucholtz and Hall locate their theory of identity firmly within the social-constructionist paradigm; identity is viewed as an emergent product of interaction rather than a preexisting condition that is constructed not individually, but in concert with all participants in an interaction. In this regard it is similar to other frameworks that regard the role of the audience and participants in an interaction as influential in the styling of discourse (e.g. Bell 1984). Furthermore, identity can be constructed from the position that individuals take up in relation to other people, and to the subjects

\footnotetext{
${ }^{4}$ This is the term with which Bucholtz and Hall refer to the field of sociolinguistics in the sense of encompassing both anthropological and variationist approaches to linguistic research. (see Bucholtz and Hall 2008b).
} 
referenced in their discourse. Thus Bucholtz and Hall draw upon (and extend) two related concepts within the field of linguistic identity construction: stance and indexicality.

\subsubsection{Stance and indexicality}

Stance may be regarded as the position that an individual takes towards a subject in discourse, such as a topic, a person or a relationship (Bucholtz and Hall 2010; DuBois 2007; Johnstone 2009). For instance, in using the discourse marker oh in reported speech, a speaker may take a stance that indicates a negatively evaluative stance towards the person or subject being reported (Bolden 2006; Holt 2000; Trester 2009). Furthermore this discourse feature may index membership of local or macro identity categories that contain this feature as part of their stylistic repertoire. Indexicality is precisely this; the verbal invocation of shared socio-cultural knowledge aligns the speaker with local components of identity such as group membership or more macro identity categories such as age or ethnicity (Ochs 1990). Taking a particular stance is one way in which this can be achieved.

However, indexicality can be analysed throughout many layers of discourse. Ochs (1990) categorises two forms of indexicality, direct and indirect. Direct indexicality is the process by which a particular feature of discourse displays an alignment with some aspect of identity that the speaker claims for themselves. These may be correlated with stance. In doing so, the speaker takes an affiliative stance (Heritage and Raymond 2005) towards that particular aspect of identity, for instance the membership of a social group (whether real or conceptual). This is the case when a linguistic feature such as the realisation of a particular vowel indexes membership of a local group (e.g. Eckert 2000; Johnstone and Kiesling 2008). However the membership of said group may index a further, macro level identity category such as age or social class, and over time the linguistic feature that signalled group identity may come to index this wider social category (Silverstein 1992). For example, using the sentence final tag eh? in New Zealand may index a number of assumptions that could be made about the speaker (all or none of which might actually be true), it may index a claim to Māori identity, an alignment with Māori communicative norms, youth or that they are simply expressing solidarity with their interlocutor (Bell 2000, 2007; Holmes 2005a; Meyerhoff 1994; Stubbe and Holmes 1995, 2000). The indexicality that is formed in the mind of the 
hearer of this utterance is dependent on their socio-cultural knowledge, their evaluation of the situation and their familiarity with the speaker. In other words, there is a wide field of possible indexicalities that rely on both local and global context for their interpretation.

\subsubsection{Ordered indexicalities}

Although indexicality is intersubjective, i.e. it is subject to potential differences of interpretation by speaker and hearer, it is possible to analyse one path of the possible interpretations of an indexicality using the concept of ordered indexicality. Silverstein (2003) categorises indexicality into not just direct and indirect indexicalities, but into an ordered series, with the first order equating to direct indexicality and the subsequent indexicalities each indexing the one previous, to the degree $n+1$. Thus a linguistic feature can index (or signal) a stance which in turn can index a membership, which can index an ideology, and so on. For example, using bro as a term of address in the rugby team indexes solidarity with the addressee, through its link to the kinship term brother. On another level of indexicality, it can index Māori ethnicity ${ }^{5}$. This may further index an ideology of "coolness" that using non-standard English confers on the speaker, especially if they are male (Kiesling 2004).

Thus Silverstein's description of ordered indexicality is the semiotic analysis of one possible framing of an indexical feature and the micro-processes that a person goes through in making the link between a linguistic phenomenon and an ideology. It does not describe the negotiation of meaning in a situation, but rather the route of a particular interpretation of a linguistic feature (Silverstein 1992). However, ordered indexicality is useful in analysing in detail the link between a linguistic feature and ideology. The analysis of indexicality in the rugby team which will be presented in Chapter 6 uses the concept of ordered indexicalities to show the link between locally negotiated communicative practices (realised as stances) and ideologies of rugby and masculinity.

The local meanings of indexicalities and stances rely on the addressee having the relevant socio-cultural knowledge to interpret a linguistic feature forming the

\footnotetext{
${ }^{5}$ Note I am not saying that it necessarily does, rather that it can.
} 
indexicality intended by the speaker. Therefore there are always multiple possible interpretations of any linguistic feature. This condition is embodied in Bucholtz and Hall's principles of relationality and partialness. These rely upon identity and stance being viewed as intersubjective, in other words interpreted differently by each person. This also holds for Blommaert's (2005) concept of layered simultanaeity in which every discursive move can be interpreted in a multiplicity of ways simultaneously.

In addition to the concept of stance as the position of a speaker towards the object 6 of their speech, stance can be viewed as the first move in the intersubjective negotiation of identity (Jaffe 2009). In other words a speaker may take a particular stance, but it is the acceptance of this stance by the addressee, signalled by the addressee's stance, that constructs an identity acceptable to both parties. Thus stance can be described as the "primary means of organising interaction" (Kiesling 2009:172). This is expressed by DuBois's (2007) notion of a "stance triangle" in which two subjects each evaluate the object of conversation and in so doing position themselves in relation to it. Their relative positions are regarded as their alignment. DuBois describes stance as "the smallest unit of social action" (2007:173); if we understand identity as being constructed in interaction (i.e. social action), then stance can be viewed as a building block of identity.

This is reinforced by the idea that the accretion of stances over time creates a particular style (Johnstone 2009). Returning to indexicality, we could say that the adoption of a particular stance indexes a style which in turn aligns the speaker with (indexes) a social group or an ideology. Therefore, the concepts of stance, style, and identity could be viewed as existing on three levels with varying distance between interaction and ideology, i.e. stance takes place in interaction, style may be expressed as an habitual stance taken in certain situations that can be referenced, while an identity is a more abstract, ideological concept. This may be compared with Coupland's (2007:113) description of three different levels of frame that can be applied to a social situation: interpersonal framing, genre framing, and socio-cultural framing. Each of these refers to an increasingly macro view of a situation: from the relationship between the speakers,

\footnotetext{
${ }^{6}$ In Dubois' terminology, the object refers to the thing on which a stance is taken. It could be a person, an idea, another stance. This can be confusing due to the other meanings of object in linguistic terminology, so other than in the explanation contained in this chapter, the term is avoided.
} 
to the norms imposed by the situation, and finally to the socio-cultural context within which any interaction takes place. As it has been established that stance is intersubjective, it may be apparent that stance is most useful in analysing authentic interaction if an interlocutor's framing of an event is known. Thus Section 2.1.3 describes framing, the process by which stance and indexicality are interpreted.

\subsubsection{Framing}

It is possible for ideologies to become associated with particular indexical features as a result of influence from cultural authorities such as the media (Bucholtz and Hall 2010). However, what should be understood is that indexicality, and the evaluation of a speaker's stance, relies entirely on their interlocutor's understanding of the situation. This is embodied in Goffman's (1974) concept of framing, which has been taken up by numerous scholars of interaction (e.g. Gumperz 1982a; Holmes and Schnurr 2005; Kendall 2004; Kiesling 2004; Morgan 2010). Goffman's concept that every participant in an interaction evaluates that interaction through the frame they impose using their socio-cultural knowledge of the participants, the setting, the function of the interaction and the past interactions of the participants is also one that is fundamental to the theoretical frameworks of the Ethnography of Communication (Gumperz and Hymes 1972) and Interactional Sociolinguistics (Gumperz 1982a).

In viewing identity (and stance) as intersubjectively constructed, stance may be described as the verbal realisation of frame, although Goffman and others (e.g. Agha 2005; Ensink 2003) use the term footing ${ }^{7}$. In signalling the way in which they interpret an interaction, a person performs an act of evaluation (in DuBois's terms) which positions them in relation to their interlocutor. Thus stance could be viewed as the meshing of frames, or the negotiation of situational understanding. However, framing can also be examined from the point of view of interpreting stances and indexicalities through the application of interpersonal, situational and socio-cultural knowledge (Coupland 2007).

Identity can therefore be conceptualised as a speaker aligning their framing of an aspect of discourse with or away from their interlocutor(s), as shown by DuBois's stance

\footnotetext{
${ }^{7}$ It is a matter of some uncertainty as to whether stance, footing, positioning, and voice are coterminous, although Ribeiro (2006) notes that all of these terms are used interchangeably by Goffman.
} 
triangle (2007). This could be seen as a possible outcome of convergent and divergent accommodation (Giles, Coupland, and Coupland 1991), where accommodation towards another person's speech style creates a stance that is aligned with that person. However, relationality is not just about aligning speaking styles, but signalling a commonality or distinction with another person. This may take the form of mimetic discourse features, but a clearer example is provided by the use of inclusive and exclusive personal pronouns (Bucholtz and Hall 2005), whereby a speaker can take a relational stance that indicates they share, or do not share, a group membership with the addressee. Research on relational stance-taking through pronoun usage has typically focused on leadership, speech-making and political positioning (e.g. Dekker 2002; Fasulo and Zucchermaglio 2002; Fortanet 2004; Wieczorek 2009). Following in this tradition, it is one of the features examined in the current research.

Like relationality, partialness can be viewed in terms of framing and it is this principle that deals with agency in identity construction. Agency is seen as the "accomplishment of social action" (Bucholtz and Hall 2005:606). It is not necessarily intentional, but rather the product of intentional discursive action and social-cultural framing. Thus every description of identity is dependent upon the context in which that identity is created; as identity (via stance) is reliant upon context so it is that unless we could describe every possible situation in which an actor may exercise the same intention, it is not possible to give a full account of identity. Therefore, as with existing research on socio-cultural linguistic identity, this research examines identity in a specific set of situations where two types of identity are constructed: leadership and group identity.

Before discussing research pertaining to these forms of identity, it is necessary to outline a further concept that is related to the notion of agency in identity construction. This is the metaphor coined by Goffman (1959) of the social situation as a stage, wherein the actors in the situation move between two areas, front-stage and back-stage.

\subsubsection{Drama and the metaphor of stage}

The metaphorical analogy of social interaction as drama is one that has been used by numerous theorists (Alexander 2004; Bauman 2000; Birrell 1981). Furthermore, identity has been described as a performance (e.g. Butler 1997; Cameron 1997; Goodwin 2000; Kotthoff 2006), and the extent to which an audience believe the 


\section{N. A. Wilson}

sincerity of the performance may be judged as to their acceptance of the identity that is performed (Goffman 1959). However, the idea of performance should not be taken literally, neither to the extent that an actor in a situation is fully in control of the part they play nor to the other extreme, that they are taking part in a fully scripted (i.e. predetermined) encounter. Rather, following the principle of partialness as outlined above, any performance of identity in a given situation is conditioned in part by a person's intentions or goals in a situation and in part by the situational norms of interaction that dictate how one should behave in such a situation (Bucholtz and Hall 2005).

Rugby is embedded within a socio-cultural ideology of performance, as are all team sports. The purpose of sport is in part to entertain, and in part to perform a ritual around which people can congregate (Birrell 1981; Donnelly and Young 1985; Goodger 1986). This performative ideology is such that it can be identified in all aspects of team culture. Every communicative event in the team can be categorised as either being performance or a preparation for performance. To distinguish these two categories Goffman's (1959) terms of front-stage and back-stage are applied. These terms are used to describe regions pertaining to performance. Front-stage is the region in which a performance takes place, back-stage on the other hand, is where the non-performance interaction takes place. This has been successfully applied to academic discourse, with the classroom as the front-stage and the staffroom as the back-stage (Richards 2010; Vaughan 2007), and to the medical encounter (Sarangi and Roberts 1999). In other words, front-stage can be seen as public or on-record and back-stage as private or offrecord. Furthermore, front-stage is used to refer to "the natural domain of the professional" (Vaughan 2007:175) which is "constructed back-stage and represented front-stage" (Richards 2006:11).

Using this definition as a starting point, this research applies the concept to the communicative events of a rugby team. However, rather than separate regions of space constituting back-stage and front-stage, it will be shown how the same space can be dynamically used as both, thus highlighting that it is the discursive activity within this space that constructs it as either front-stage or back-stage. This departs slightly from Richards' (2006) use of the terms, as he makes a distinction between front/back-stage and front/back region, with the former describing the actual space of the interaction while the latter refers to the behaviour itself. Due to the fluidity of space in the rugby 
club, in which the same space can be used for multiple different activities, specific spaces cannot be ascribed permanent status as front-stage or back-stage. Rather, they take on this aspect in light of the action that takes place and the audience that is present. In other words, the space is defined by the discourse that takes place in it (Mondada 2009). Furthermore, the term stage emphasises the idea of performance and preparation inherent in rugby ideology and thus a part of the socio-cultural framework of rugby. While Richards (2010:147) points out that there has been some criticism of Goffman's use of the stage as an analogy for performance of everyday interactions, in the case of the rugby club it is entirely apt, due to the degree of performativity inherent in a sports team.

As noted above, following the principle of partialness, it would be impossible to give a full account of identity in any social group as one would have to consider every possible context in which interaction could occur. Therefore, this research does not attempt to fully describe every aspect of identity in the rugby team, but focuses on two different types of identity construction: the construction of a person as a leader and the construction of a group identity that defines the team as unique. As will be discussed here, and then shown through the analyses presented in this thesis, these are both accomplished through social (linguistic) practice. The following section describes existing research on the first of these, leadership.

\subsection{Leadership}

Leadership is a topic that has been studied across a myriad of disciplines, each with their own definition of what leadership is and how it is done. However, following the theory of identity in interaction outlined above, the approach taken here is that leadership identity (i.e. an identity as a leader) can be viewed as a sustained repetition of stances that can be interpreted as leader-like. The problem lies in defining what is meant by leader-like discourse. It is this problem that the study of leadership discourse addresses. Using authentic interaction as a basis, much research has been carried out not only on what constitutes leadership discourse, but the different discourse strategies that people use in performing effectively as leaders (e.g. Agho 2009; Holmes 2005b; Holmes and Marra 2004a). Unlike leadership psychology (e.g. Bono and Judge 2004; Gordon 2007; Vroom and Jago 2007) and management studies (e.g. Goleman 2000; Groves 2005; Mandell and Pherwani 2003), leadership discourse research does not 
internalise leadership as a collection of character traits, or indeed make any assumptions about whether some people are more likely that others to emerge as leaders. Leadership discourse research essentially sees leadership as a practice, a view that is also espoused in several studies arising from organisational studies (e.g. Busher, Hammersley-Fletcher and Turner 2007; Crevani, Lindgren and Packendorff 2010; Drath and Palus 1994; Raelin 2011).

Until now, the only research carried out on the discourse of sports leaders has focused on the description of coaching as a register (Heath and Langman 1994). While the introduction to this thesis underlined why I consider sport a valid area for sociolinguistic research, it should also be understood that sport, in particular professional sport raises questions about what constitutes work and what constitutes leisure. A professional sports team is undoubtedly a workplace if work is defined as the occupation by which one earns a wage (Rigauer 1981). However a more inclusive approach might look at a sports team in organisational terms in that there is often a rigid hierarchy and set roles for individuals within the team. In this regard it is comparable with many forms of workplace and thus the lack of research on leadership in sports teams may be made up for by the existing research on leadership that has taken place in workplaces.

A good deal of contemporary research on leadership discourse arises from the study of workplace communication, in particular Victoria University's Language in the Workplace Project (LWP 2010). Other research that focuses on workplace leaders has been undertaken in the UK (Mullany 2007), Hong Kong (Chan 2007; Schnurr and Chan 2009), Japan (Saito 2009; Takano 2005), and Denmark (Ladegaard 2011b), showing that this is an area of research of international and cross-cultural relevance. Under the aegis of workplace research is included research on academic institutions (Chiles 2007; Richards 2006; Vaughan 2007), medical institutions (Harres 1998; Sarangi and Roberts 1999; Waitzkin 1990), government departments (Holmes, Stubbe and Vine 1999), as well as commercial organisations (e.g. Chan 2007; Holmes and Marra 2004b; Ladegaard 2011a; Mirivel and Tracy 2005; Mullany 2007; Murata 2009; Vine et al. 2008). Using the rationale above, sports teams could be added to this. 
In addition to the view that a sports team can be considered an organisation that can be compared with workplaces, there is a link between amateurism and professionalism in sport in that many amateur sports players aspire to become professionals. For some this is a realistic goal. The rugby team studied here contains such players. Example 2.1 is an extract from an interview with Mozza, a member of the player leadership structure, and shows that while this ambition is present among many of the players, it is not something that is talked about openly.

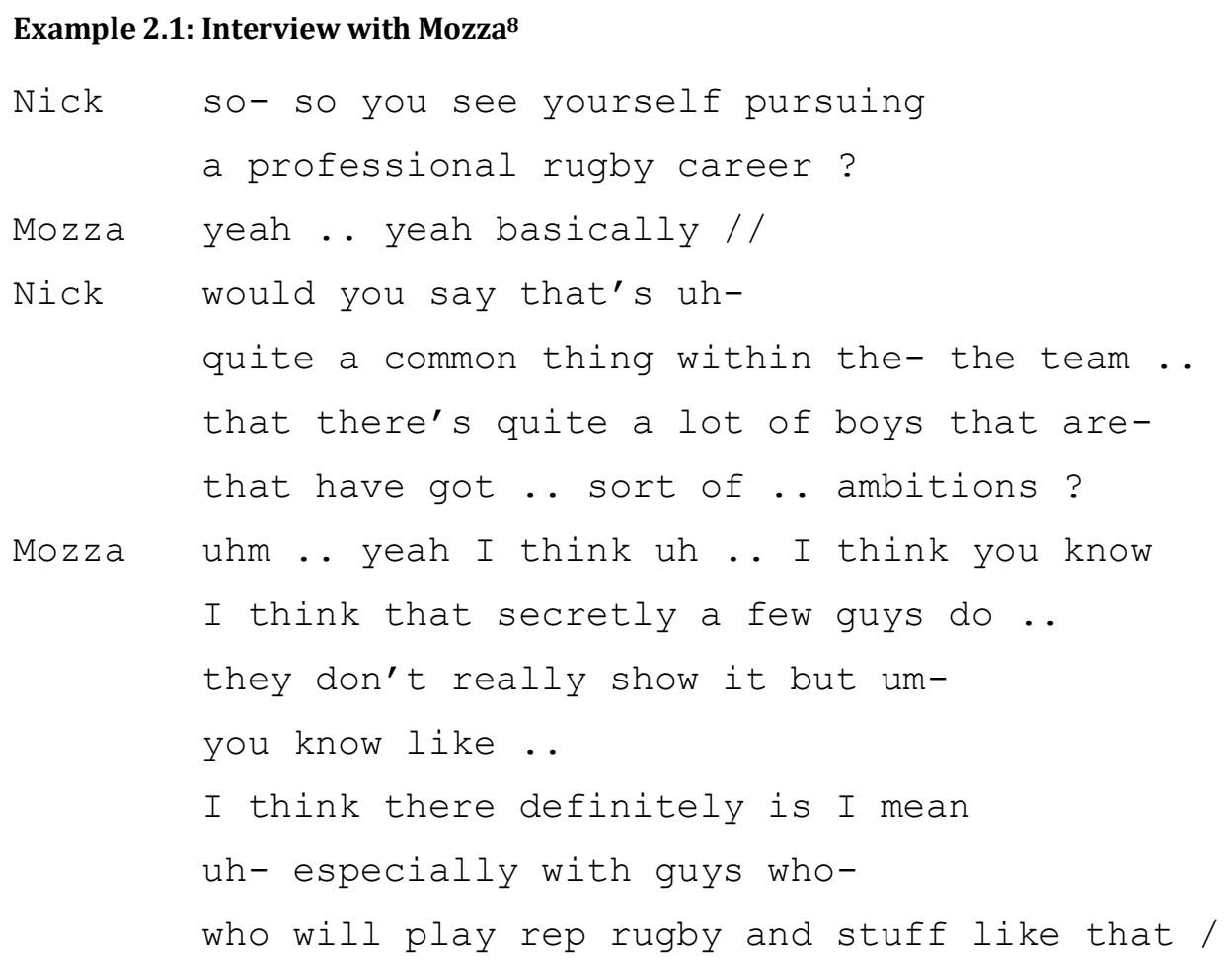

The reticence about discussing one's ambitions is consistent with the socio-cultural background to workplace and leadership norms in New Zealand. The "tall poppy syndrome" is referred to in a number of studies of Antipodean cultural norms (Peeters 2004), indicating an unwillingness to admit to aiming high for fear of being criticised as undeserving of success. This discursive norm can be compared with Māori cultural norms, which although being more concerned with knowing ones place in a social order and showing appropriate deference, results in the same reluctance to reveal any ambition to achieve lofty goals (Holmes, Vine and Marra 2009; Schnurr, Marra and Holmes 2007; Watson 2007). As is discussed at several points in the following chapters, 


\section{N. A. Wilson}

New Zealand and Māori socio-cultural norms have an influence on the way in which leadership is performed in the rugby team under investigation in that they provide a cultural context within which local identity can be constructed. In an international context, the differences in leadership styles between cultures, for instance New Zealand and Hong-Kong (Schnurr and Chan 2009) or the UK and China (Spencer-Oatey 2005), and the intercultural miscommunication that can result in the differing interpretations of leadership behaviour have also been an area in which leadership discourse has been examined.

It is however the area of gender on which the vast majority of research on leadership discourse focuses (e.g. Baxter 2010; Holmes 2005b; Mullany 2010; Vine 2004), often in combination with ethnicity (e.g. Holmes 2009; Holmes, Marra and Vine 2011; Holmes et al. 2009; Schnurr et al. 2007). It may seem that the examination of external factors on leadership discourse is at odds with the social constructionist paradigm in which the conceptualisation of identity described in the previous section fits. However, rather than seeing the particular way in which leadership is enacted as being dependent on these external factors, contemporary research in this area takes the view that the way in which leadership is performed indexes higher level ideologies that are influential in creating an identity. Thus by employing particular discourse strategies to perform leadership, one constructs oneself not only as a leader, but a leader with a particular cultural background and gender. For example, using an unmitigated directive to a subordinate may index a stereotypically masculine leadership stance (Vine 2004), regardless of whether it is used by a woman or a man. The impact that this has on the creation of a leadership identity is that a female leader who frequently employs this stance may be regarded as having a leadership identity that is seen as less feminine (Baxter 2010; Holmes and Schnurr 2006). Holmes (2006, 2009) gives several examples of the leadership identities that can be created through the use of gender-linked leadership stances. For women gendered identities that can be indexed in performing leadership are roles such as queen or mother (Holmes 2006; c.f. Mullany 2010), while for men some archetypal identities are the hero, father and good mate (Holmes 2009). It should be remembered however that these are locally constructed identities and have been created in concert with the other members of the leader's workplace. Nonetheless approximations to these styles can be found in many workplaces and thus they are a 
useful point of comparison when analysing the way in which leaders create their identity in discourse.

Research in the area of gendered leadership discourse has successfully debunked the myth that female leaders always do leadership in one way and male leaders in another (Holmes and Marra 2004b). However, stereotypes still exist at an ideological level and can be indexed in the course of enacting leadership. One such ideology is that female leaders pay more attention to the relational needs of their subordinates, while male leaders are rather more blunt and adhere to discourse that focuses on transactional (task-oriented) interactions (Ladegaard 2011b; Mullany 2007). Relational Practice (RP), the maintenance of relationship within an organisation pursuant to organisational objectives (Holmes and Marra 2004b), is a strategy that has come under scrutiny in research on gender in leadership discourse. While Fletcher (1999) describes it as being "disappeared", positioning RP as a stereotypically female discourse strategy, other research (Holmes 2005a; Holmes and Marra 2002a; Holmes and Schnurr 2006) shows that in fact men and women are just as likely to use RP as each other. Indeed Holmes and Marra (2004b) use gender neutral pseudonyms in the presentation of their workplace data to show that, counter to the stereotype, male leaders can use RP and female leaders can use direct, authoritative styles of leadership discourse. This does not mean that a leader always uses a particular style of leadership. Just as the stance that a person takes in an interaction is highly dependent on context, so is the leadership stance (or style - there is some conflation of the terms in the literature) dependent on the situational context, the leader's objectives and the social norms of the organisation.

As noted above, current thinking in this field suggests that although leaders may index gender stereotypes as a resource for constructing a leadership identity, effective leaders of either sex tend to use a balance of relational and transactional styles of leadership discourse (Holmes 2005b; Holmes and Marra 2004b). This leadership strategy may be expanded beyond the individual leader, with leadership as a co-operative or joint activity. This is an idea with growing currency in the field of leadership and management studies, as well as in leadership discourse. Just as identity is constructed by all participants in an interaction, leadership is jointly constructed by leaders and followers (Crevani et al. 2010; Morgeson, DeRue and Karam 2010; Raelin 2011); furthermore, leadership activities can be shared among a number of co-leaders (Heenan 
and Bennis 1999; 0’Toole, Galbraith and Lawler 2002; Vine et al. 2008; Wilson 2009a). Whether this takes the form of co-leadership, which incorporates a hierarchy of leaders and deputy leaders, or shared leadership which is a flatter model of leadership, the idea is collectively referred to as distributed leadership (Bolden 2011). When an organisation contains a distributed leadership structure, different leadership styles may be used by the leaders, perhaps with one leader focusing on transactional needs while the other mitigates this with attention to relationship within the organisation (e.g. Wilson 2009a).

Having multiple leaders in an organisation is of interest from an analytical point of view because in performing leadership, leaders must not only interact with followers but also negotiate how they practice leadership with their fellow leaders. This aspect of leadership is examined most closely in this thesis; i.e. the idea of leadership as a negotiated discursive practice that is not only negotiated in its front-stage enactment to followers, but in back-stage encounters between leaders. This thesis thus aims to contribute to the field of leadership research by giving an in-depth description and analysis of a distributed leadership structure and the way in which its members negotiate their leadership roles and define through discourse what constitutes leadership practice in the rugby team. Viewing leadership as a practice is an idea that originates from the Communities of Practice model in which a group can be categorised by the activity around which it is centred rather than the characteristics of its members (Wenger 1998a). This model is described in detail in the following section in which the link between group membership, identity and language is established.

\subsection{Communities of Practice}

First posited by Lave and Wenger (1991) in describing a theory of situated learning, and more fully developed in Wenger's later work (1998a), the concept of Communities of Practice (CofP) has been adopted in many disciplines, not only as a theory of description in the social sciences, but as an organisational tool in business. Sociolinguistics could be said to have been an early adopter of the CofP framework ${ }^{9}$, when Eckert and McConnell-

\footnotetext{
9 The terms "theory" (Holmes and Meyerhoff 1999; Wenger 1998a), "framework" (Eckert and Wenger 2005), "model" (Holmes and Meyerhoff 1999; Moore 2010a), "concept" (Moore 2010a; Wenger 1998a), "construct" (Eckert 2000; Moore 2006) and "approach" (Moore 2010a) are all extant in its description in the CofP literature, and many scholars use some of these interchangeably as the references to existing
} 
Ginet (1992) used the theory to extend their analysis of language and gender. Since then it has been utilised by many researchers to analyse the linguistic practices of social groups (e.g. Daly, Holmes, Newton and Stubbe 2004; Eckert 2000; Holmes and Schnurr 2005; Rock 2005; Schnurr 2008a; Tusting 2005).

The CofP model is posited as an alternative to at least two other ways of analysing groups of speakers: i.e. as a social network and as a speech community (Holmes and Meyerhoff 1999). Where the CofP theory differs from these approaches is that rather than grouping individuals purely by their sustained interaction with others, as in social network theory (Milroy 1987), or only by a shared set of norms and/or linguistic variables, as with speech communities, it uses a combination of these, respectively labelled mutual engagement and shared repertoire by Wenger (1998a), along with a third, joint enterprise. Mutual engagement refers to the interaction between members of the CofP; shared repertoire is the set of norms, linguistic behaviours, fashions etc. that are held in common by the community; and joint enterprise refers to the goal or purpose around which they form. Thus each of these dimensions is related to the other two: the group are engaged around some enterprise and in the course of this engagement with each other shared practices, or ways of doing things, emerge. As Eckert and Wenger (2005) are at pains to point out, in response to a commentary on the sociolinguistic use of the theory by Davies (2005), the CofP is an analytical tool, not a social construct and it is highly unlikely that its members would define their membership of the group (if they see a group as existing) using these criteria.

The three dimensions listed above tend to be considered the basic tenets of CofP theory and, as used by some, the theory begins and ends here. However, integral to CofP theory is the notion of identity and in this respect there are similarities to social identity theory (Holmes and Meyerhoff 1999). Unlike social identity theory, CofP theory not only builds in a goal (i.e. the joint enterprise), but also presents individual identity and group identity as being mutually dependent. In other words, the identity that each member

research show. My use of these terms reflects this. It may be noted that many recent scholars avoid using any term at all, referring just to the CofP, perhaps reflecting the disagreement about what the CofP is. Arguably, the CofP approach, to use a relatively neutral term, is both theory and model, depending on how it is used. The details of how it can function and account for social practice may be regarded as theory, while the description of what constitutes a CofP is a model. There is however some degree of overlap between these usages. 


\section{N. A. Wilson}

brings to the group helps to shape the group's identity and in turn the identity that is jointly constructed by the members impacts upon identities that they present to others.

Eckert's (2000) seminal study of student CofPs at Belten High School is often cited as the prime example of the CofP theory as used in sociolinguistics. It combines an ethnographic method of discovery with linguistic analysis of the shared practices within the CofPs identified at the school. This is then related to wider patterns of sociolinguistic variation in the Northern United States and, most importantly, to the way in which the shared practices co-construct individual and community identity for the CofP. It is the link with community identity which makes the CofP model a useful one for the purposes of analysis of the rugby club, as one of the goals of this research is to determine how discursive practices construct an identity for the team.

It is perhaps the notion of a shared construction and continual re-negotiation of practices that has not only made CofP theory attractive to sociolinguists (Bucholtz 1999; Eckert and McConnell-Ginet 1992; Holmes and Meyerhoff 1999), but for organisational management scholars as well (e.g. Brown and Duguid 1991; Drath and Palus 1994; Garrot-Lavoué 2009), because the process of change as a positive aspect is built into the theory. The fact that a CofP can change its practices through the introduction of new ways of doing things by members is both a strength and a weakness. While this changeability allows for many different types of CofPs to be defined and for them to remain CofPs even when their make-up and practices alter, it also lays the CofP model open to criticism about how members decide to adopt certain practices (e.g. Davies 2005).

In her critical analysis of the use of CofP theory in sociolinguistics, Davies (2005) questions the degree of agency CofP members have in adopting features that signify membership of a CofP. This highlights what Davies sees as a shortcoming of the theory, i.e. it does not address the question of whether a person purposefully adopts a certain linguistic feature in order to join a group. However, in response, Eckert and Wenger (2005) stress that CofPs are a method of description not discovery, and hence the idea that an individual displays some form of agency in becoming a member of a CofP misses the point somewhat. While an individual may intentionally join, for example, a social group in a high school through sustained mutual engagement (e.g. Bucholtz 1999; 
Eckert 2000; Moore 2010a), they can only be said to have become a member of the group in its conceptualisation as a CofP once their social behaviour conforms to the group. They themselves may class themselves as a member prior to this and the other members of the group may or may not think of them as members of their group. At this level what is being described is a social network (Eckert 1988; Milroy 1987). While there may indeed be a degree of intentionality to adopting the group's practices, this is not done with the purpose of joining a CofP but to display a stance of solidarity with people with whom they have a desire to associate (Bucholtz and Hall 2005). As analysts of sociolinguistic behaviour we can categorise such a group as a CofP, but with the proviso that members and practices may change, altering the way in which we describe it as such (Moore 2010b). Having said this, there clearly exists awareness on the part of the members of a CofP that they belong to some form of social group, and that they share values and practices. Therefore, although the CofP model is indeed an analytical construct, it is one that reflects a social fact. Members are aware that they must maintain their engagement in the community in order to prevent marginalisation (see Example 6.4), but would probably not explain this in such terms.

The CofP theory allows for the description of internal movement and different degrees of alignment in a group defined as a CofP. By analysing an individual's membership of a CofP as showing greater or lesser alignment to the practices that define the CofP, they can be categorised as closer or further from the core of the CofP (Wenger 1998a). A member's alignment can change over time, either as they adopt more of the core practices, or the defining practice of the CofP changes. This can be described using the concept of a trajectory. A member may thus be described, for example, as peripheral but on an inbound trajectory, meaning that they do not currently exhibit all of the core practices that are part of the CofP's repertoire, but have adopted more of them over time and thus, if this continues, may in time be described as a core member.

Thus a CofP is not a static entity; it has a definable beginning and the criteria for membership, the practices that take place within the community, are constantly negotiated and reified. However, that is not to say that all members of a CofP have equal negotiation or "meaning making" rights. The theory allows for a hierarchy to exist within a CofP, with higher status individuals having greater influence over what is accepted as shared practice (Eckert 2000; Eckert and Wenger 2005). This is a point that 
Davies (2005) makes, suggesting that further investigation is needed to examine the nature of hierarchy and leadership in the legitimisation of CofP membership. Following Davies, my research questions the role of the leader in a CofP, which Wenger (1998a) links to that of a peripheral member and broker between CofPs. As is shown in Chapter 4, my analysis of the CofP model as it is applied to the rugby club is that leaders are in fact core members of CofPs rather than peripheral.

It should be remembered that any hierarchy is relative to the CofP; a person whose communicative practice constructs them as a leader in one CofP may not be evaluated in the same way by another CofP of which they are a member (c.f. Section 2.3). This is crucial to my research as the rugby club is not only rigidly hierarchical as an organisation, but contains multiple, overlapping CofPs, each with their own power structure. This research is unusual in that it examines a CofP from its inception, describing how it forms and changes over time. A CofP begins with "[a]n aggregate of people who come together around mutual engagement in an endeavor" (Eckert and McConnell-Ginet 1992:464). In the case of the rugby team, this endeavour is to play rugby competitively ${ }^{10}$ and the players come together for this purpose at the beginning of the season. This is the formation of a team CofP, in which the coaches are the core members, and of which the players gradually become members through their conformity to the practices established by the coaches, which are:

- A commitment to attend every training session.

- Displaying positionally appropriate rugby skills during these sessions.

From the squad of players that has come together, those that display the greatest level of conformity to these two practices will be selected by the coaches to form the Premier Team, which is the team that is studied in depth in this research. The players not selected by the Premier Team coaches, will become the lower level team, the Senior Ones, or if young enough, the under twenty-one team, the Colts. Throughout the course of the season, players from either of these two teams may be called up to the Premier Team (henceforth the Prems, their own name for themselves) to replace players who

\footnotetext{
10 One might think that rugby is by definition competitive. However a competitive team, in which results really matter, must be considered differently to a social team, in which the focus is more on enjoyment, exercise and getting to know others.
} 
have been injured. Thus there is a continual introduction of new peripheral members to the team and their interactions with other team members may serve to establish them as regular members of the Prems and thus integrate them more fully into the CofP.

The formation of the team CofP is not the end of the process however, nor is the team's evolution limited to a negotiation of the practices that define it as a CofP. As the first competitive fixture of the season approaches, new, smaller CofPs develop based around the way that training sessions change to focus on positional aspects of play. The players split into two separate groups determined by their field position, Forwards and Backs. These groups train separately, the Forwards with the head coach, who was a Forward himself in his playing days, and the Backs with the assistant coach, who was likewise a Back. Due to the separation of the groups in the training session, and different forms of engagement, each group develops practices separate from one another, thus becoming CofPs in their own right. As the season progresses further, smaller CofPs emerge, again through additional practices that are shared by a different configuration of players and coaches. In Chapter 4, the composition of these CofPs, their practices and their realisation in discourse is examined in detail. However, it should be noted at this point that the way in which CofPs can be seen to develop within a bounded temporal continuum, i.e. the rugby season, is not necessarily reflective of the way in which they might operate in other social contexts. For instance, many CofPs, such as those found in workplaces, are ongoing, and could theoretically last indefinitely. They might have a gradual influx of new members who learn the social and communicative practices necessary to align with the group and although practices may change over time, the CofP in effect remains the same as there is a continuation of practice negotiation. In the Prems, by contrast, the end of the season (described in detail in Chapter 7) marks the dissolution of the team and due to the break in engagement and an often drastic change in personnel between seasons, the following season's team begins the process of CofP formation anew.

As multiple CofPs develop within the Prems, the same is happening in other teams in the rugby club. The club as a whole can therefore be considered a Constellation of Practice (Wenger 1998a), that is, an organisation containing multiple CofPs which may or may not overlap just like Eckert's Belten High School (2000). This concept has been further developed by others such as Rock (2005) who uses it to describe a police force 


\section{N. A. Wilson}

with the CofPs that occur through the practices that take place in and around interviews with suspects, and Brannan (2007) who described the way that sexuality is constructed in a call centre. This concept can be used as a way of bounding a group of CofPs that may not fulfil all the dimensions of a CofP, for instance mutual engagement. While all of the members of the Prems are mutually engaged, a player in the Prems is highly unlikely to have any contact with a member of the J8s, the social team, or the coach of one of the children's teams, despite all of these individuals being members of the club. A key ingredient of the Prems' identity is the club that they represent, just as the school they go to is a key ingredient in the Jocks of Belten High's identity. This contextual information must be incorporated into the CofP. It is through the Constellation of Practice that this is accomplished.

A key part of the CofP framework is that it must be informed by ethnography (Eckert and Wenger 2005; Gee 2005; Meyerhoff 2005). Thus, the CofP model is a useful analytical partner to an ethnographic approach such as Interactional Sociolinguistics, demonstrated by the numerous studies that have used the two in concert (e.g. Bucholtz 1999; Chan 2007; Daly et al. 2004; Holmes and Stubbe 2003a; Kendall 2004; Vine et al. 2008). While some scholars (e.g. Creese 2005; Davies 2005) have pointed out the limitations of the CofP model in accounting for detailed negotiation of linguistic practice, others (Eckert and Wenger 2005; Tusting 2005) have suggested that there is scope for building on the CofP model using Linguistic Ethnographic approaches. The research detailed in this thesis aims to do just this, by expanding upon the existing conceptualisation of how CofPs form from social or institutional groups.

The next section (2.4) builds on the exposition of terminology and theoretical frameworks to articulate the research questions that are addressed in this thesis. As noted above, ethnography is vital when using a CofP approach and when examining the situated interpretation of events. Section 2.5 explains how an Interactional Sociolinguistic method can be used to research the topics outlined above and further, why it is an appropriate methodology to use in answering the research questions outlined in Section 2.4. 


\subsection{Research questions}

As the title of this thesis suggests, the focus of this research is leadership and team identity, analysed within a CofP model. These are closely linked and each draws upon the others, as exemplified in later analysis chapters. However, for clarity's sake, the research questions have been divided into three separate questions as follows:

1. How do members of a rugby team construct themselves as leaders through discourse?

2. What discourse strategies are used by leaders to perform leadership?

3. How does the team construct a group identity and what role do leaders play in this?

Question one is concerned with the emergence of leaders and the role of the individual in performing a stance aligned with leadership practice. The second question asks what these leadership practices are, while the third question expands the focus from leaders to the whole team and the way in which a group identity is constructed. Each is thus concerned with identity and leadership, but in different ways. Furthermore, each can be addressed by examining the communicative practices that exist within the team. To this end, and following similar approaches to research on socio-cultural identity and leadership discourse, I use Interactional Sociolinguistics (IS) as my main methodological framework. The background and principles of this framework are specified in the next section, highlighting the importance that is placed on ethnography as a research tool.

\subsection{Interactional Sociolinguistics as a methodological framework}

IS evolved from the Ethnography of Communication/Speaking (Gumperz and Hymes 1972) and ultimately from the linguistic approaches of mid twentieth century North American anthropology (Blommaert 2005; Bucholtz and Hall 2008b; Gumperz and Cook-Gumperz 2008). It draws upon anthropological tradition in that cultural as well as situational context is seen as crucial in interpreting communication (Gumperz 1982a). This interpretive approach to analysing discourse is enabled by ethnography, another way in which IS draws on anthropological methodology. Furthermore, due to the cultural-relativist origins of linguistic anthropology, IS and other related approaches analyse language in terms of its local communicative efficacy (Blommaert 2005). In other words, IS examines how a communicative system does the work it needs to do 
within the linguistic ecology in which it operates (Gumperz 2005). It is when different systems of communication come into contact that miscommunication arises, as shown by the many IS studies on intercultural miscommunication (e.g. Gumperz 1982a, 1982b; Holmes et al. 2009; Spencer-Oatey 2005; Tannen 2005); what works for one system does not necessarily function in the same way in other systems. Thus participants in a communicative event interpret what is going on based on their past experience of similar events. They use contextualisation cues such as prosody, intonation and reference to other events as well as the spatial and temporal situation of the event to determine what the function of the communicative event is. This is the process of framing (Goffman 1959, 1974; Gumperz 1982a) which has already been briefly mentioned in regard to identity and the interpretation of stance. Individuals from different cultural backgrounds are likely to frame the same communicative event in different ways based on their past experience of similar events, creating different expectations of what specific linguistic behaviour means in the context of this interaction. Thus a feature of language such as placing a final falling tone on the word please in the utterance exact change please, may be interpreted by the British hearer as impolite, while for the West Indian speaker it simply follows the prosodic norms of their cultural background (Gumperz 1982a:168-170). It is the analysis of these contextualisation cues, informed by ethnographically acquired insight that is the focus of IS.

IS has been described as having an "eclectic toolbox" for analysis (Bailey 2008:2317). This is what makes it such a useful approach. Rather than taking linguistic form as the starting point for analysis, IS follows its anthropological heritage and addresses linguistic function by paying close attention to the context of language (Gumperz 2005). In other words, IS is an approach that can be used to examine how a particular function of discourse, such as leadership construction, is achieved. In doing so, it examines different discourse strategies that achieve this function and how various linguistic forms are used in their creation. For example, in workplace research IS has proved a fruitful methodology for analysing leadership in communities of practice (e.g. Holmes 2005a; Holmes et al. 1999; Mullany 2010; Richards 2006; Schnurr 2008a). By conducting close ethnographic study of a specific workplace, contextual factors such as socio-cultural norms, membership of local CofPs and established repertoires of 
appropriate behaviour can be taken into account. Thus it is possible to analyse how the discursive enactment of leadership is shaped by contextual factors. IS utilises analytical methods from conversation analysis, but rather than viewing micro-aspects of discourse such as emphatic stress or overlapping speech as constitutive of meaning in themselves, it looks upon them as indexing contextual information, thus creating a local practice in which they signal the speaker's stance in interaction (Gumperz 1999). Such indexical features are known in IS terminology as contextualisation cues and can be seen as the way in which speakers signal stance and the information that hearers use to determine frame. However, contextualisation cues are not limited to prosody and turn-taking; they can also be lexical or phrasal, such as with discourse markers (Bolden 2006; e.g. Kiesling 2004; Schiffrin 1987).

In signalling a relational leadership stance that emphasises solidarity, for example, a speaker could use in-group jargon, familiar terms of address to other group members and references to shared history while the content of their speech may perform tasks related to the goals of the organisation. In the rugby team this can be seen in the way in which the coaches issue instructions to the players in training sessions (see Chapter 5). Furthermore the use of discourse markers, prosodic and intonational features can signal evaluative stances to each participant's discourse, providing a clue as to the power relationships that are enacted in an event like this (Bucholtz and Hall 2005; Heritage and Raymond 2005; Trester 2009).

An IS approach to analysing leadership discourse can thus focus on a particular function of speech acts that are frequently employed in enacting leadership, such as control acts (Vine 2004), or humour (Schnurr 2008b). In the latter case, it would be impossible to successfully analyse humour as a strategy used in performing leadership without establishing what constitutes humour in a particular workplace. It is for this reason that ethnography is an essential component used in IS research. As interpretations of linguistic behaviour (i.e. frames) are formed through experience of similar interactions in similar (or the same) context, IS analysis begins by finding repeated situations such as these through ethnography (Gumperz 1999). Such situations are referred to in IS as communicative (or speech) events. Communicative events are bounded in space and time, governed by social norms and participant roles which constrain what speakers can and cannot do in that event (Gumperz 1982a:165). Often there are names for these 


\section{N. A. Wilson}

events in the linguistic repertoire of the culture in which they exist. In the rugby team this is manifest in the naming of specific communicative events, which have ritual significance (described in Chapter 3), as well as communicative events that can more accurately be described as occurrences of a particular speech activity, such as planning the execution of a training drill (see Chapter 7). The identification of these events and the acquisition of the necessary knowledge of local practice to interpret them in as close a way as possible to the participants is the crucial role that ethnography plays in IS. The following section situates IS within the wider fields of Linguistic Anthropology and Linguistic Ethnography and begins the discussion of the importance of ethnography to sociolinguistics, which provides the starting point for the description of methodology in Chapter 3.

\subsection{Linguistic Anthropology and Linguistic Ethnography}

Ethnographic insight gained through long-term, first-hand immersion in strategically selected fieldwork situations is applied to the interpretation of what transpires in longer sequences and yields hypotheses on how native speakers think in everyday interaction. IS is one of several traditions concerned with these issues." (Gumperz 2005:215)

IS is but one of a number of analytical frameworks which make up the "loose alliance of work" referred to as Linguistic Ethnography (Bucholtz and Hall 2008b:424). Linguistic Ethnography is for the most part synonymous with Linguistic Anthropology, in that the former derives from the latter and espouses the same methodological approach. The difference in label is reflective therefore not of a change in theoretical or methodological stance, but in situating Linguistic Ethnography as the form of Linguistic Anthropology practiced in "late-modernity" (Rampton 2006; Tusting 2005). Regardless of label, what is crucial in anthropological approaches to linguistics is the use of ethnography as a means not only of collecting authentic spoken data, but also acquiring the relevant local socio-cultural knowledge with which to interpret it (Bucholtz and Hall 2008a; Gumperz 2005; Hammersley 2007).

Considering ethnography as a useful, even vital, tool for sociolinguists provides a good starting point from which to examine how ethnography can be used effectively 
(Rampton 2007a). However, as ethnography is an anthropological methodology, it is anthropological literature that provides the most useful discussion of how ethnography is best carried out. In anthropology the practice of ethnography is important in itself and the ethnography is seen as the product of research rather than as simply a tool for acquiring information that can be used to inform a more micro-level analysis (Atkinson and Hammersley 1994). However, the adoption of ethnographic method by linguists utilises many of the same fieldwork techniques. Linguistic Ethnography may go further in the recording of spoken interactions and the subsequent linguistic analysis of these recordings, but the methodological issues surrounding the data collection and fieldwork are very similar and so it is important to address these issues in describing the data collection methodology used in this research.

When conducting ethnographic research it is possible to become overly immersed in the research site and lose the detachment that is crucial in analysing social groups. This problem is extensively addressed in the ethnographic practice literature (Agar 1996; Atkinson and Hammersley 1994; Hammersley and Atkinson 1983; Walsh 2004) where a four-fold typology of participant-observation is often used to describe the options available to the researcher: complete observer, complete participant, participant as observer and observer as participant. There appears to be common agreement that the ideal position for an ethnographer is somewhere between participant as observer and observer as participant. In practice this means starting as an observer and getting more involved with the social group until one reaches this desired in-between status.

However, there comes a point at which the researcher must take a step back or risk losing the ability to look at the research as a researcher rather than as a member of the community (Hammersley and Atkinson 1983). The rapport that is built up between the researcher and the participants, while necessary, can hamper the researcher in analysing interactions from an outsider's point of view. In other words, conducting ethnography necessitates a duality of identities on the part of the researcher; they must be both insider and outsider, not a participant observer but both participant and observer (Agar 1996).

An alternative to a researcher becoming a participant is to have an existing insider become the researcher. This approach can be useful as research may proceed more 
quickly because entry to the research site and integration with the participants are, in effect, already complete (Holmes and Bell 1988). Although these two approaches start from opposing positions - the outsider acquiring the view of an insider and the insider acquiring the view of an outsider - they share the problem of striking a balance between one's responsibilities as a researcher and one's affinity with the participants (Hammersley and Atkinson 1983). It is thus vital for an ethnographer to maintain some distance between themselves and their participants. Even if already familiar with the community being researched, the ethnographer's objective should be to look at situations with fresh eyes, to make the familiar strange (Agar 1986).

There is a distinction between Linguistic Ethnography and linguistic studies that briefly utilise participant observation. Using participant-observation as a data collection tool is not ethnography (Schensul, Schensul, and LeCompte 1999; Wolcott 2002; c.f. Feagin 2002). Linguistic Ethnography is as much about acquiring the socio-cultural knowledge necessary for "native-like" interpretation as it is about collecting authentic data (Edge and Richards 1998; Sarangi 2007; Wolcott 2002). Just as important in defining Linguistic Ethnography as a research framework is Cameron et al.'s (1992) strong case for researching with, not on, the participants and this is an important feature of my research. In other words, I considered it important to let the participants lead the way not just in being fully aware of the objectives of the research, but to actively participate in shaping its outcome in terms of goals and analysis, in addition to the development of the recording methodology (see Chapter 3). This consultative principle is one that I have wholeheartedly embraced in my methodology and, I feel, adds extra value to my research.

In addition to including participants input in research design and objectives, ethnography also allows the researcher to put aside their preconceptions about what might be found in a community. While they may be looking for certain behaviour, perhaps based on some prior knowledge of the community, ethnographic methodology encourages researchers to build theory from the data they collect. Thus ethnography can be viewed as a Grounded Theory approach (Charmaz and Mitchell 2001). Grounded Theory is the discovery of theory from data, rather than the verification of theory using data. This approach was initially developed by Glaser and Strauss (1967) for use in sociology but has subsequently been widely adopted throughout the social sciences 
(Boeije 2010; Charmaz 2001), including Linguistic Ethnography (Creese 2010; Johnstone 2000). Charmaz and Mitchell (2001) suggest that ethnography can gain structure and discipline using a grounded theory approach, one of the main tenets of grounded theory being the early analysis of data while data collection is still underway. This allows the researcher to shape the ethnographic approach to account for insights gained from this early analysis, giving the data collection a focus. The movement between data collection and analysis can re-occur many times and forms the basis of the spiral of analysis (Boeije 2010), an important principle in qualitative analysis. In IS, this is reflected in the way in which ethnographic work informs the selection of the communicative events that will be analysed. By analysing the data that is collected as the research is carried out, further targeted recordings can be made. In the current research, this approach led to the targeted recording of specific events on match days following the early analysis of observations and recordings of a whole match day. This allowed much more efficient time-management during fieldwork, when more time could be spent on making detailed observations and notes, as well as during transcription of the match data when the laborious process of searching the recordings for transcribable events had in effect already been carried out. The details of this however, are explained in the next chapter in which my methods of ethnography, data collection and analysis are fully described.

\subsection{Summary}

This chapter has introduced the concepts that are used in undertaking the analyses of communicative events in the rugby team that form the body of this thesis. I have discussed how leadership can be analysed both in terms of identity and in terms of social communicative practice. These two views of leadership complement each other as both can be used to explore the way in which leaders communicatively perform as leaders and how they emerge as leaders. Furthermore, since identity can be said to emerge from practice, the three concepts of leadership, identity, and practice are closely intertwined and it is only by analysing the way in which they are all enacted and constructed through language that a fuller picture of leadership in the rugby team can be put together.

While this chapter has addressed the main concepts that inform this research, these will be developed further in the course of the later analysis chapters, using more specific 


\section{N. A. Wilson}

research to support the conclusions that are drawn. Thus, not all of the literature that will be referred to in the course of this thesis has yet been discussed. This has been done to underscore the ethnographic nature of this research, and allows for this thesis not only to provide an analysis of how leadership is enacted in a rugby team, but to tell the story of the research that has been conducted and the journey taken over the course of the season spent with the team.

Chapter 3 begins this story, showing how an ethnographic approach was developed and also the methodological decisions that were taken in the course of gathering the data and acquiring the knowledge that could be used to interpret this data. 


\section{Chapter 3 Methodology}

As outlined in the introduction to this thesis, while the analytical framework of this research is Interactional Sociolinguistics, the methodology for data collection is rooted in ethnography. As described in the review of the literature supporting my theoretical framework, Interactional Sociolinguistics is embedded within Linguistic Ethnography (Creese 2010; Rampton 2007b) and the process of ethnography whereby a researcher gains close access to research participants is not only a way to record authentic linguistic data, but is also a means of developing the researcher's own understanding of the Community of Practice with which they are working. This is because the process of carrying out ethnography reflects the path followed by a new member of the CofP. As this research shows, through sustained interaction with the CofP the researcher can become a peripheral member. This chapter explains how this was achieved and the data collection and methodology that made it possible.

\subsection{The Role of ethnography}

Ethnography is not just about gaining information, but about changing understanding (Agar 1996; Hammersley and Atkinson 1983; Walsh 2004). Ethnographic research does not see the observer's paradox as a problem (c.f. Feagin 2002); in fact it is embraced (Eckert 2000; Wolfson 1976). Not only does ethnography change the participants of the research, it also changes the researcher. In other words, ethnography is crucial to both data collection and analysis as it is through ethnographic contact that one experiences events with the participants and can ask them questions with an insider's status about what meaning those events have for them. This builds understanding and allows the researcher to find meaning in interaction in as participant-like a way as possible. When later analysing the recorded discourse the researcher can draw upon the insights gained through ethnographic participation to interpret events as a member of the community of practice. This approach (i.e. gaining the perspective of a CofP member) is an attempt to overcome what Sarangi (2004) terms the analyst's paradox, where the researcher cannot attain a participant-like interpretation of events without being a participant. As this chapter demonstrates, an important part of this was my own identity as a former rugby player, as I share similar lived experiences with the participants in this research. 


\section{N. A. Wilson}

Through Linguistic Ethnography, both participants and researcher gain knowledge about the way a community works, and may discover new communicative practices that become part of the community's discursive repertoire. As noted in the previous chapter, both Linguistic Ethnography and Interactional Sociolinguistics are often used to analyse Communities of Practice as they can provide the understanding of social context that is the basis for the interpretation of interactions that take place within a CofP (Bucholtz 1999; Geyer 2009). Linguistic Ethnography espouses an approach of research with, not just research on (Cameron et al. 1992; Rampton 2007a). Not only does it allow the research participants to influence the course of the research through feedback, but by being aware that they are participating in research on communicative practices, the participants may become more self-aware of their communication and (in the case of this research) leadership practices. This may lead to both individuals and the CofP as a whole creating new practices, or re-negotiating existing ones, in the light of the knowledge they acquire. This idea is explored in detail in later sections of this chapter.

As is often the case with ethnographic research, I use a grounded theory approach (Allan 2003; Charmaz and Mitchell 2001; Komives et al. 2006). Data collection and analysis were not discrete events; a preliminary analysis and description of recordings was carried out as soon as possible following sessions, as was the composition of my ethnographic field notes. Analysis and fieldwork were therefore integrated for a large part of the research process. This is described in grounded theory literature as the spiral of analysis (Boeije 2010). Thus, through constant analysis of new information, an ethnographic approach was developed and adapted in response to the situations in which I found myself. As Hammersley and Atkinson state, "research design is crucial to ethnography, but it is a reflexive process that operates throughout every stage of a project" (1983:21).

This chapter details the chronological development of the research methodology, showing firstly how this research grew out of my previous research on a rugby team and how this, combined with reference to the existing literature on ethnographic practice, informed not only the design of my data collection methodology but also my role as an ethnographer. Following this is a description of how access was gained to the rugby team, how recordings were gathered and how events were selected for recording. This section also includes the design of feedback sessions that were conducted at two 
stages of the research: with the coaches at the end of the season, and the following season with the players. Finally the relationship between analysis and fieldwork is discussed to illustrate that when doing Linguistic Ethnography and Interactional Sociolinguistics, there is little division between the two.

\subsection{Research design}

\subsubsection{Pilot study}

Much of the design of this research grew out of prior research I had carried out for my Master's thesis, which investigated leadership discourse in a Scottish rugby team (Wilson 2007). In that research I practised what might be called participant immersion (Agar 1996) in that I actually played for the team that I was researching. The lessons learnt from that research greatly informed the design of the current research both in terms of what worked and what did not. As such my Master's research acted very much as a pilot study for the purposes of the methodological design of the current project, at least as regards data collection. However, there are notable differences. First and foremost, the current research has a wider scope in that it investigates not just leadership but team identity. In addition, the current research involved recording not just training sessions (here with multiple simultaneous recordings) but also match days.

It was not possible to play for the team in New Zealand as I aimed to research a higher level of rugby and unfortunately, although having played rugby to district level in Scotland when younger, I have neither the skill-level nor the physical presence to successfully achieve this in New Zealand. Furthermore, from a research point of view, I found that while conducting research as a player, I was unable to gain access to as many aspects of team behaviour as I would have liked. This was because the same norms of interaction that constrained other new players applied to me, and I did not have the freedom to question the coaches as fully as I would have liked.

\subsubsection{Constructing my role as an ethnographer}

The problem of how to gain entry to a rugby club in New Zealand without being a player or a New Zealander was resolved by talking to club officials and coaches, and being overt about the fact that I wanted to research their linguistic behaviour, but making sure that it was understood that I was also a former player and thus an insider in terms of rugby culture. This was useful not only as it allowed me access to a team more easily 
than would have been possible for a non-rugby person, but also because I was already privy to many of the key understandings that are used in interpreting rugby team interactions. However, at the same time I was an outsider, being a non-New Zealander, and this made it acceptable for me to question the coaches and players about what they said and did without losing face. Over time, I also took part in team activities more and more, such as by carrying training equipment or filling water bottles. I thus occupied the ideal ethnographic position of both insider and outsider, participant and observer (Agar 1996; Atkinson and Hammersley 1994). In short, I became a peripheral member of the community of practice.

This is central to the development and understanding of this thesis, for without my intensive commitment to ethnographic research I would have neither data to analyse, nor the deep understanding of the workings of the team that underpins the analysis of the interactions that I recorded. In fact I would argue that in order to fully understand the inner workings of any CofP one must follow the same path that any new member follows. The CofP is a model concerned with situated learning and the development of meaning, and just as a person gains membership through learning the social practices necessary to display membership, so must the researcher learn these practices (Gee 2005; Lave and Wenger 1991; Wenger 1998a). As Penelope Eckert most notably demonstrated in her seminal research on high school students in Detroit (Eckert 2000), it is vitally important that a researcher hoping to gain an authentic perspective on what interactions mean to CofP members first gains the trust of their participants. This is achieved most easily by conforming to the norms of interaction that are part of a CofPs repertoire, and define its identity. For instance, Eckert aligned herself with the students at Belten High principally by not aligning herself with the teachers. By not maintaining a classroom presence, she was able to create an identity on the fringes of the CofPs that existed in the school, trying to be accepted by both but not being able to "win acceptance within one group at the expense of another" (Eckert 2000:77). In essence, she gained the trust of the students because she was in the same position as them: "in the school on the teachers' and administrators' sufferance, and had to be careful not to annoy teachers, not to be in the wrong place at the wrong time, [and] not to cause trouble" (2000:73). 
I approached the issue in a somewhat different manner, due to the less rigid power asymmetry that exists in a rugby team compared with a school. Rugby players are there by choice, they play because they want to and while the coaches may exercise power by not allowing them to play rugby, the balance of power between coaches and players is much more delicate than the teacher-student asymmetry noted in Eckert's research. Furthermore I was dealing with an adult CofP. I indexed my identity as a former rugby player in many of the early interactions with players and coaches, using a common discursive strategy found in rugby teams, the narrative of injury (Fenton and Pitter 2010; Howe 2001). I established common ground with players and coaches and explained my own non-participation in playing rugby by recounting an ankle injury I sustained in university rugby, which effectively ended my participation in rugby ${ }^{11}$. Not only did this convey the message that I was a rugby insider, it also showed me performing a discursive practice that is part of a rugby player's repertoire, in this team as much as any other. This was just one of the ways in which I aligned with local and global rugby practices in order to construct an identity as a rugby insider.

Although the focus of this thesis is on the discursive practices of the team, also of use to me in constructing an identity as an ethnographer was the way in which I dressed, and the props I used. For instance, it soon became clear that I should wear a tracksuit when with the team at training sessions, but smart clothes (number ones) on match days, as this is what the team did (c.f. Eckert 2000). This was also quite practical because my close proximity to the players at training sessions often meant that I would be standing in what was essentially a large patch of mud. However I wanted the players to remember that I had a non team role, as a researcher and to this end I constantly wore a recording device around my neck, whether I was using it or not. In the early days of my fieldwork I often stood apart from the players (just out of earshot) and made notes using the recorder; often it was not that I needed to make the notes, but that I wanted the players to see me doing something that they might think of as "research". The other reason for doing this was that the players and coaches would become used to the idea that there would often be a microphone present, even though at this stage they were not being recorded.

\footnotetext{
11Until my return to playing rugby for the purposes of my Masters research which was at a lower league level than previously.
} 
The difference between the new member and the ethnographer is that the ethnographer must also maintain some analytical distance, otherwise they risk "going native" (Agar 1996). In order to ensure this, I took a break of three weeks from the team during the mid-point of the season. Not only did this allow me the space to maintain some distance, but it also gave me an opportunity to transcribe much of the recorded data I had gathered by that stage and perform the analysis that was necessary in order to more precisely target which events to record. In practice what this meant was that the more time I spent with the team, the less I needed to record, because I was able to precisely identify not only what I needed to record, but also when it would happen, knowing from experience what was a ritual event and what might be a one-off (Rampton 2006:392). This was accomplished through a preliminary analysis of the initial sessions I recorded, comparing them in terms of event structure. From this it could be established which events would be most likely to provide useable data.

I found that not only were the structures of the match days and the training sessions highly formulaic, but so were the events that took place within these sessions. The formulaic nature of both the different types of sessions and of the events that were recorded is described in Section 3.4. The next section describes how I gained access to the club and the process of beginning the ethnography.

\subsubsection{Gaining access}

As mentioned previously, the reason that I chose rugby teams as a research site stems from my own past experience as a rugby player. First however, I had to gain access to a rugby team. Following the example set by other practitioners of participant observation (e.g. Agar 1996; Holmes 2000; Kiesling 2006; Milroy 1987) I sought a personal connection as an entry point to a rugby team.

This turned out to be through friend of a friend, Barry ${ }^{12}$, whom I met soon after my arrival in New Zealand. When Barry first asked how I came to be in New Zealand, I told him about my research - then in its very early stages of planning. It turned out that he coached a children's team at a local rugby club and he promised to help me make contact with relevant people. Three months later, once my research proposal and ethics

12 All names have been replaced by pseudonyms. 
application had been approved, Barry spoke to the club president on my behalf. This led to a meeting between me and Tommo, the head-coach, who was enthusiastic about the research and allowed me as much access as I wanted to the team. Having negotiated timescales with Tommo I began fieldwork in March 2009, when the three teams that make up the senior club were still in pre-season training. This allowed me to see the development of the Prems, the team I would most closely work with, from its very beginning.

\subsubsection{Initial observations and interviews}

Before commencing any situational recording, I followed a more traditional (observational) ethnographic approach. This involved attending as many training sessions and matches as I could, talking to coaches and players and generally getting a feel for the rugby club. It was through finding out how the team functioned as a Community of Practice and the routine social interactions that took place within this that not only did I develop my recording strategies, but also laid the groundwork for my acceptance as a peripheral member of the group. In addition, it provided a means for me to seek the views of the participants about my research, giving them a very early opportunity to influence its focus, thus fulfilling the goal of doing research with participants, rather than on them (Cameron et al. 1992).

Just as Eckert (2000) did not approach students at Belten High until her presence was ultimately accepted, making instead some observational notes about their immediately apparent social practices, I wanted to familiarise myself with the sociolinguistic ecology (van Dijk 2008) of my research environment before attempting to gain the trust of the players. Therefore, for my first few weeks with the team I restricted my informants to the coaching staff and the squad managers as they had been my first points of contact and acted as gatekeepers (Anderson 2007) for my entry into the team as a researcher. By gaining the coaches' trust, I demonstrated to the players that I was an "official" observer, even if at the early stage they were a little unclear as to my precise role. Therefore, at the first training session that I attended, although I gave a brief introduction to the assembled players about who I was and what I was doing there, I concentrated on talking to coaches individually. I let the players see this, hoping that this would help them relax and accept my presence at training sessions. 
In addition, as one of the foci of this research is leadership, it made sense to concentrate on the relationship between the coaches before investigating the players. In order to do this I conducted an informal interview with each coach when they were away from the players and other coaches. Nevertheless, targeting the coaches in my interviews did not prevent the players approaching me to find out about what I was doing. The players that approached me in this fashion early on in the fieldwork remained interested in the research throughout and were very useful at various stages, not least when testing the recording equipment, as detailed in Section 3.3.2. This contributed to the players' roles as co-researchers, consistent with Sarangi's definition of "consultative research", as "a collaborative exploration of the nuances of professional practice" between the researcher and "professional practitioner" (2005:373-4)

From the initial period of ethnographic study I was able to build a clear picture of the hierarchy of the club and the relationship between the three teams which comprise the senior club, before concentrating on one team, the Prems. The Prems were chosen as the focus of the ethnography because they represented the level of rugby that while still amateur, was a step for some players on the path to professional rugby; indeed during my time with the Prems, two of the players made the step up to professional status with provincial teams. Thus the players in the Prems could be viewed as taking their rugby seriously, and having aspirations to make a career from playing rugby. This perception was confirmed in interviews with players.

Over the course of my ethnographic research on this team I conducted several interviews with the Premier team coaches and, as I became more and more integrated into the team, I held short interviews with the players who were part of the leadership structure. Although these interviews were initially intended only as a guide for me in determining the structure of the team, they later provided valuable data contributing to the analysis of the way in which leadership identity was constructed, as well as the attitudes that the various leaders within the team had towards the process of leadership itself. The design of these interviews is described in Section 3.2.4. Starting with this broad view of the club as a whole allowed me to make sense of the wider context of interactions between players and coaches. As knowledge of the club structure forms a part of the contextual information that players use to frame communicative events 
within the team, it was crucial that I too gain as much of this knowledge as possible (Hymes 2003).

Following the initial interviews and after attending training sessions and matches for eight weeks, I judged the players to be ready to be recorded. This was indicated by their relaxed manner when talking to me and the interest in the purpose of my research that had been shown by a few of the players. However the most striking marker of my acceptance into the team was that the players asked me to join the huddle. As will be shown later in this thesis, the huddle is an important site for performing and negotiating leadership identity, and membership of the team. Therefore, to be included was a strong indication of how the players saw my place in the membership of the team and this acceptance was what facilitated the recording of authentic spoken data within the Prems. The next section details with the actual process of recording, including the equipment used, the events selected and the ethical and logistical considerations that had to be taken into account.

\subsection{Recording}

\subsubsection{Ethics}

Prior to any fieldwork taking place, ethics approval was sought from the VUW Human Ethics Committee. Information sheets and consent forms were designed and the ethics of the research were approved. It was made clear in both the consent form and the information sheet that any participants reserved the right to withdraw from the study at any point and any recording of them would be deleted. Participants were also given the opportunity to listen to their recordings if they wished. None of the participants either withdrew or asked to listen to the recordings, although several were interested in being informed of the findings, as detailed in Section 3.3.2.

Aside from consent, the key considerations regarding ethics were safety, anonymity and confidentiality. The question of safety arose due to the need to attach recording devices to players whilst they were training. Initially I also hoped to record them during matches, but it was decided at an early stage that this was unfeasible, because it carried too great a risk of injury both to the player carrying the recording device and to the opposition. Furthermore, there was the problem that the opposition teams might see the recording of on-pitch communication as an attempt to record the codes they use for 
communicating pre-arranged moves which could be considered cheating on the part of the Prems. It was however decided that with enough padding, and with thorough player consultation, a safe method of fitting the players with recorders could be devised, and thus the recording of players during training sessions could be achieved.

As regards anonymity, pseudonyms were adopted from the outset in any documentation regarding the team that was intended for anyone outside the team and this was made clear on both information sheets and consent forms. In terms of confidentiality, it was a matter of trust between myself and the team that I would not reveal strategically important pieces of information that are kept secret, such as codes for lineouts, names of moves etc. Again, it was stressed that any such information would be scrambled where it appeared in any documentation. Outlining how these issues would be dealt with at the stage of gaining consent for participation allowed me to build a bond of trust with the team.

At every match or training session that was recorded I made it clear that recording was taking place. When events were being recorded in the Team Room there was a recorder hanging from the ceiling in clear view of all the players and when I recorded using a handheld recorder I held it out in front of me, despite the recorder being quite capable of picking up the players' voices when simply hanging round my neck. During the training sessions however, it became apparent that the players sometimes forgot that they were wearing a microphone. When able to, I checked with the players, giving them the opportunity to review and request any deletions, and they were happy to let these segments remain. However, more detailed analysis of the recordings uncovered parts of the players' conversations that I judged to contain sensitive information. These were marked by a player making some statement that conveyed that they had forgotten about the recorder such as don't say that, I'm being recorded. I took these as clear signs that the conversation surrounding this should be treated as confidential and so these segments of the recordings were replaced with white noise (so as to keep timings consistent).

Conversely, on some occasions it was obvious that the players and coaches were fully aware of the recorder, speaking directly to it; using the recorder as a way of putting their achievements "on the record" as it were. This is illustrated in Example 3.1. 
Context: Training has just finished, Bug and Will are having a kicking competition.

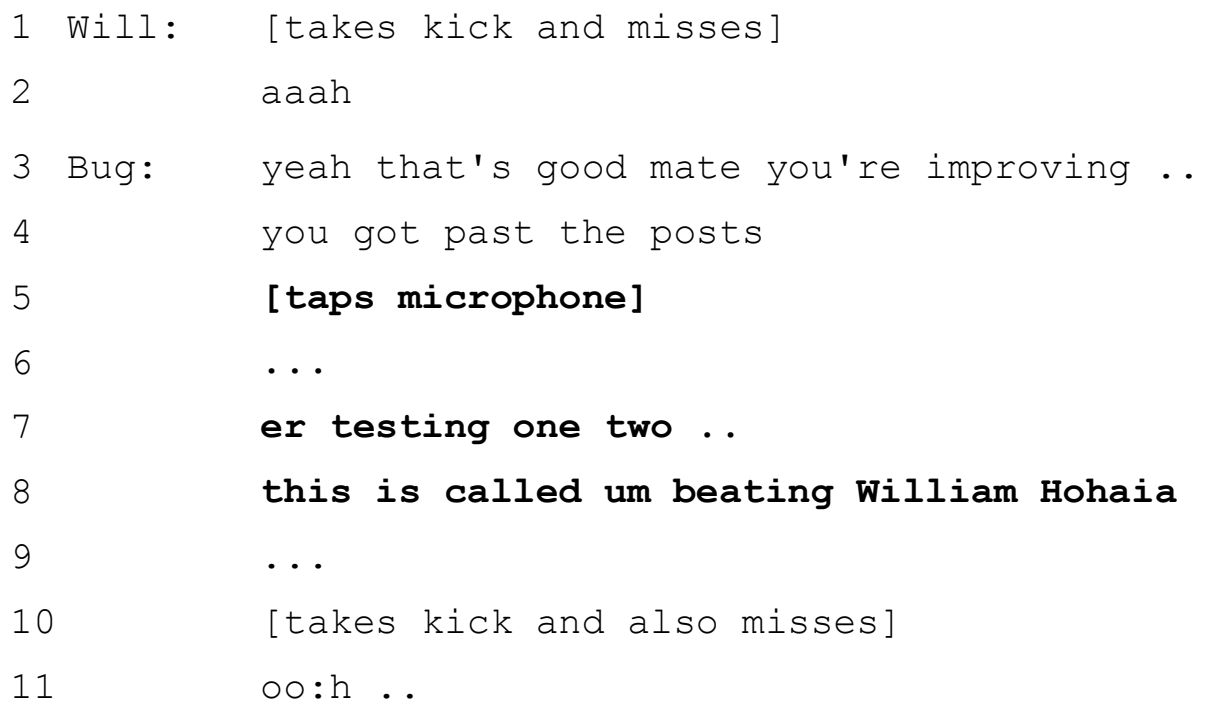

It is interesting that the players used the recorder in this way, as a discursive prop to aid in what is in this case a display of one-upmanship. This is a phenomenon that has been noted by Wolfson (1976) who sees the recorder as functioning as a participant in the conversation. Alternatively, following Bell (1984), one could ascribe the recorder auditor or overhearer status, in that the speaker is aware of it and modifies the way they speak in order to accommodate to a wider audience. I take the view that it is in fact a marker of the adaptability of speakers to use any available resource to help them create a social identity through discourse, and that not only is Bug addressing an external audience to put his speech "on record", but he also uses the microphone (along with a nasal quality to his voice) to index a style that is reminiscent of sports commentators. Thus, awareness of the recording equipment gives the players an extra dimension through which they can display their stance in an interaction, by treating it as a non-participating audience. The extract above is analysed in more detail in Chapter 6.

On occasion it was unavoidable that non-participants were recorded. For instance, during a training session it was not uncommon for various people affiliated with the club to approach Tommo while he was wearing the recorder and hold a conversation with him. I developed three strategies to cope with this. Firstly, I had shown both 


\section{N. A. Wilson}

Tommo and Parky how to stop recording if they wished. If they felt that a conversation was truly confidential, they could prevent it from being recorded. Secondly, if the person that was recorded was a member of the club staff from whom I did not already have recording consent, I approached them afterwards and gave them the option to either sign a consent form or for the part of the recording that featured them to be deleted. All those approached in this manner gave their consent. The third option was to delete the person from the recording and this was done for people to whom I was unable to speak subsequently in order to gain their consent.

\subsubsection{Recording equipment}

Prior to engaging in fieldwork I aimed to record full training sessions, pre-match lockerroom interactions and matches using multiple microphones on players. As noted above, it soon became apparent that recording players' on-field speech during matches was not feasible from an ethical or a logistical standpoint. Firstly, there was the potential for an injury to occur to the player wearing the device or (more likely) to an opposition player that might hit the device in the course of the game. This was less likely in training sessions, and furthermore I would be able to monitor the equipment more closely and remove it easily if it became a hazard. Secondly, it seemed likely that the microphone would record not only the players on the participant team, but also whoever they were playing. In addition to these ethical obstacles was the question of whether it was worth expending the time and resources to record a situation that might not yield much in the way of data that could not be gathered in other ways. This decision proved to be wellfounded given that in some of the training sessions large parts of the recording were untranscribable due to the combination of multiple simultaneous speakers, wind, and other background noises. I observed that on-field interaction during matches was very similar to this. I had also intended to record the coaches during matches and training sessions, and decided to go ahead with this using the tried and tested method of a digital voice recorder with a lapel microphone attached (Holmes 2000; Wilson 2007). As will be explained, once the development of the player recording equipment is described, I used my growing understanding of the team to identify precisely which events involving the coaches would yield the most coherent source of spoken data.

Although the idea of recording players during matches had been discarded, I still planned to record the players during training sessions. However, recording equipment 
had to be found that would enable the best compromise between player safety and recording quality, with the first of paramount importance. To devise a solution I compiled the following requirements with which a potential recording solution must comply:

1. The recording device must not have the potential to injure the player wearing it or any other player.

2. The device must not interfere with the player's movement or ability to play rugby.

3. The device must not get damaged from physical contact with other players or the ground.

4. There should be no wires which could be pulled loose during movement and contact with other players, as this could present a hazard to the player (or damage the recorder).

5. The microphone must be sensitive enough to pick up the player's voice as well as surrounding players yet shielded from wind noise as much as possible.

6. The device must be secure, i.e. the recordings must not be openly transmitted as this would contravene my ethical requirement of confidentiality.

7. The device should not be too expensive as there was always a possibility that it could be damaged.

Initially, radio microphones were considered the best option, but these did not satisfy requirements 3 and 6 . A radio microphone system that would satisfy requirements 1 to 6 , would have involved a prohibitively large amount of money (I was quoted NZ\$20,000 for ten days hire of the equipment). To get a microphone which was small enough without being too expensive I had to build it myself from components available in kit form. This worked well. However by the time I had encased it in plastic and neoprene it was the same size as my voice recorder. Furthermore, upon more rigorous testing I identified a major problem that made it unusable. It did not work well under impact because the broadcast frequency jumped each time it was hit.

Having rejected the radio microphone as a viable means of recording the players, I began experimenting with a self-contained device in the form of a digital recorder attached to the player. The only problems that this presented were satisfying 
requirements 1 to 3 and these were resolved with the aid of some neoprene padding and an mp3 holder that fitted round the arm. This device was further developed as I acquired a much smaller recorder which could be worn on an arm support. All of this was done before I began the fieldwork, during which consultation with the players led me to refine further the way that it was attached to the players.

I began working with participants by talking to one of the injured players who was standing at the side of the pitch watching the training. It transpired that he had badly injured his wrist in a hunting accident and would not be able to play for eight weeks. Nonetheless he appeared at training each night. I mentioned to him that I would be attaching microphones to players and asked for his opinion on where best to wear such a device. He gave me some useful feedback which I used to further modify my designs for constructing a harness for the device. He suggested that rather than wearing it on the arm, the device be attached across the chest or the small of the back. I also asked some other players what they thought of these options and they agreed that these would both be good positions as they would not hamper players' movement.

Using the players to help develop my recording equipment is one indicator of how I adopted a consultative, participant-led approach to my research from the start and I made clear to them their importance in helping shape my methodology (Sarangi 2005). Using the players' input to my recording device design, I constructed a harness that would securely attach a small $\mathrm{mp} 3$ recorder to the torso, incorporating plenty of protective padding. Satisfied that this would fulfil safety and comfort requirements, all that was left was to test the quality of the recordings.

\subsubsection{Testing}

In order to test the recording device and harness I sought the aid of the team captain, Jon $^{13}$, with whom, by this time, I had established rapport. We decided that the device would work best underneath his jersey and this would not only be most comfortable for him, it also effectively eliminated any wind noise.

\footnotetext{
${ }^{13} \mathrm{~A}$ full list of the participants and their respective roles in the team can be found in Appendix B. All names are pseudonyms.
} 
We started with the device on Jon's chest and halfway through training moved it round to his back. Jon said that he found both comfortable but if he had to choose he'd opt for the back position. However, on listening to the quality of the recordings, it turned out that while the back position recorded the surrounding players well, the chest position was much more effective at picking up Jon's voice. As it was important to capture the voice of the wearer, I decided to opt for the chest recorder position. One problem that we discovered during this test was that Jon did not feel comfortable wearing the harness during scrum practice as it interfered with the way the props and he bound together as the front row. This was solved by attaching the recorder to the scrum machine ${ }^{14}$ while scrum practice took place. Another problem that was not immediately apparent was that the recorders were not waterproof. Thus, when it rained I had to remove the recorders from the players. Eventually one of the player recorders did in fact break due to water damage, although I suspect that this was not from rain but from sweat, another factor I had not considered previously.

By now I had obtained four recording devices thus allowing me to record training sessions with a device on the head coach, Tommo, and the assistant coach, Parky, as well as two player devices, which I decided would be fitted to a different player in each session in order to capture the interactions of as many players as possible. For the coach devices however, things were simpler. They simply put the device in their pocket and attached a lapel microphone. The next section explains in detail how the recording devices were deployed and the rationale behind the decision regarding which events to record.

\subsection{Situational recording}

\subsubsection{Match days}

As mentioned above, although the initial plan was to record players during the matches, this was not possible. I thus concentrated my efforts on the coaches on match days. However, in doing this I discovered that it was not what happened during the match that was important from the point of view of leadership identity, but what happened at

\footnotetext{
${ }^{14}$ A scrum machine is a semi-portable construction made up of pads and metal or wooden supports weighed down with some form of ballast, in this case several non-participating players and one researcher. It is used for the forwards to practice the timing and binding they need win in a scrum. See the glossary in Appendix A for further detail and pictures.
} 
certain key points throughout the match day. These were all identifiable as discrete communicative events with a function, a start, an end, and as explained below, a label (Hymes 2003; Wolfson 1976). Although these communicative events had been noted during the observation period of the fieldwork, their significance was only discovered upon recording Tommo and Parky for one whole match day. From listening to (and writing a description of) the events that were recorded, I discovered that a large amount of what was captured during the three hours of recording was the coaches' interactions with people outside of the team, such as fans, coaches of other teams and referees. While this was very interesting from the point of view of how the coaches constructed their identity as coaches and displayed their membership of the team to the outside world, it was not usable data for this research as I could not obtain consent from the people to whom the coaches spoke; by the time I was aware of who had been recorded, it was too late. Additionally, my focus from the start was how leadership and team identity are constructed within the team, not how the coaches represent themselves to the outside world.

Despite the large volume of unusable material that this first trial recording session produced, it did allow me to confirm two earlier observations. One, that the interactions within the team that could reliably be defined as speech events on a match day, and two, that they could be transcribed (i.e. not indecipherable, fragmented, overlapping snatches of speech, which typified most locker-room small talk when it was recorded). These events were highly ritualistic; not only did the same events occur at the same time, in the same place on every match day, but internal components of these events were also formulaic. This important point is discussed in more detail in Chapter 7. The communicative events that were identified are as follows, and are listed in the order they would occur on a match day:

- $\quad$ Team Meeting

- Pre-match Huddle

- Half-time Huddle

- Post-match Huddle

The ritual and formulaic nature of these communicative events plays an important role in the way in which team identity is constructed within the team, as well as providing a 
platform for the team leaders to address the players. Consequently, the content and linguistic structure of these speech events provide a large part of the data upon which the discussion contained within this thesis rests, and as it will provide the focus of subsequent chapters will not be discussed here other than to say that it is the repetition of these events, week on week, that made them stand out as important events to record. Furthermore, from a practical point of view, it required only one microphone to record these events as they were the few times that the players and coaches all stood (or sat) in the same place and talked for any length of time. The Team Meeting and Pre-match Huddle took place in the team room, a multi-purpose room which served as changing room, meeting room and after-match socialising space. This allowed me to attach a microphone to the ceiling directly above where the Tommo stood when addressing the players for the team meeting and where the pre-match huddle took place. The fact that these events always took place in the same spot in the team room is further evidence of their ritual nature. Recording in this way was also necessary as the Pre-match Huddle was the only huddle I was never invited to join, and it would not have been appropriate for me to do so, as it was made up only of the players who would take the pitch.

During matches I sat on the reserves bench, making notes about how the game was progressing in order to contextualise what was said later (and to give me something with which to start conversations with players after the match). As the season progressed I also carried water to players on the field when requested, or re-filled water bottles and ice-packs at the side of the pitch. At half-time, everybody on the bench, including me, ran over to wherever the players who had been on-field at the end of the first-half had congregated, and a huddle formed. I held a voice recorder in the middle of the huddle, while being part of it, which successfully captured all that was said. The same process was repeated for the full-time huddle.

However, this does not mean that I focused purely on these events. I was aware that concentrating solely on ritualised events would not give a full picture of the sociolinguistic practices of team (although as discussed in Chapter 7, these are highly important events for both leadership and team identity). As an ethnographer I was aware of the need to be adaptable (Hammersley 2007; Sarangi and Candlin 2003). Furthermore, the recording of events such as huddles would only show the front-stage (Goffman 1959; Richards 2006), where practices were performed, while the negotiation 
of practices frequently took place back-stage. Therefore, when it came to recording unpredictable events that I judged relevant to leadership or team identity, I had to be somewhat opportunistic. Often I had observed such events several times, but been unable to record them on other occasions. For example, during the last match of the season, I was standing next to Tommo when he asked an injured player to relay a message to the team when he ran on with water for them. I quickly took off the recorder that I habitually wore around my neck, started it recording and asked him to wear it when he ran on. By this stage I was so much a part of the team that he did so without pause. I was thus able to capture a speech event that I had hitherto only observed: the relayed instructions of the coaches to the players. I was interested in how the player might re-interpret them and as he was a leader within the team himself, what leadership style he might use to do this. These opportunistic recordings however, were the exception rather than the rule and were only possible once I was familiar enough with the team and the structure of the match day to predict what might happen next. The training sessions, however, while following a pattern to a certain degree, were nowhere near as ritualistic or formulaic as the match days, and for this reason I attempted to record as much as I possibly could, as detailed below. This is where I gathered the majority of what I have categorised as back-stage interactions.

\subsubsection{Training sessions}

Whilst in the Match Day interactions I identified a set of formulaic communicative events that included the whole team (with relatively little interference from background noise) in the Training Sessions, I found that these sessions had fewer formulaic structures in their communicative events. Additionally, the team split up into small groups and then recombined at various stages throughout the session. As I became more familiar with the training, and indeed as a routine was established by the team, I saw that although splitting into groups was predictable, the training activity (and thus the speech events that these entailed) would be much more varied than on match days. Although I had already developed a method for recording the players during these sessions, it was only through the careful observation of how the sessions were run that I could work out who should wear a microphone.

As detailed extensively in the next chapter, which describes the way in which the team is composed of multiple overlapping CofPs, the players and coaches regularly split into 
two of the positionally defined CofPs, Forwards and Backs, for training purposes. As shown in later chapters, this entailed a difference in the shared negotiation of practices in each CofP and thus I knew that it was crucial to record what happened in both. Therefore, I opted to place one player recorder in the Forwards and one in the Backs as well as having recorders on both Tommo and Parky. To decide which players within each CofP would wear a recorder I targeted different aspects of my research question on each session I recorded. For the first recorded session I wanted to record officially sanctioned leaders among the players, and therefore positioned the recorders on Jon, the senior captain (in the Forwards) and Bug, the vice-captain (in the Backs). For the second recorded training session I had a recorder on Mozza, the co-captain (in the Forwards) as he was the remaining official player leader that I had not recorded and in the Backs I recorded a relatively new player, Afi, in order to see how he conformed or not to the established norms of the team. On the third and final training session that I recorded I placed the recorders on two players who I had observed performing leadership behaviour but were not officially recognised as leaders within the team hierarchy. In the Forwards this was Mason and in the Backs, Smithy.

Although the player recorders captured a huge amount of spoken data, much of it was untranscribable due to wind noise, the noise of players running, or differences in volume between speaker and addressee (often only one side of a conversation could be heard). Much more reliable were the recorders that the coaches wore, and for every session but one both Tommo and Parky wore the recorders for the whole session. For one session Parky arrived late and I decided to take this opportunity to put the recorder on one of the squad managers, Duncan. I had hoped that he would behave as he usually did and spend much of the time engaging in small talk with injured players, a task I had noticed him do on several occasions. Unfortunately, he spent most of the time in the clubhouse talking to the managers of the other senior teams about club administrative matters instead and when Parky did eventually arrive, I could not find Duncan to reclaim the recorder. This however, is one of the risks of ethnography; sometimes things do not go according to plan. It is one of the key principles of ethnography to improvise and seek out any data sources that may seem like a good lead and sometimes this results in valuable data, other times not (Hammersley and Atkinson 1983). The extended timeframe within which most ethnographic studies take place allows 
ethnographers to take these risks, as there are often many such opportunities. Linguistic Ethnography faces an additional challenge in that the data must be clearly recorded and this was a challenge I faced regularly, balancing the opportunity to record new situations with the quality of the recording that would result. It is perhaps this last factor more than any other which led me to depend primarily on huddle-based interactions as these produced the clearest recordings.

As the coaches spent much of the time in the midst of the players, their microphones picked up the majority of interactions and the player recordings were used to fill in gaps. The total duration of training session recordings that this multi-recorder approach resulted in was around twenty-two hours, and after each session I listened to the recordings and wrote a description of the events captured on each recorder. I then synchronised the four audio files from each session into multi-track format so that a session could be played and the sound from each recorder turned on or off as the recording of the session was replayed. This provided several microphone feeds on many conversations, with speech that was missed by one microphone often picked up on another. By combining the descriptions that had been made of the recording from each microphone and my field notes, I was able to identify what could be regarded as discrete communicative events. These were events that involved more than one speaker, spanned more than one turn and, from the early analysis of ethnographic observations, seemed to function in creating leadership and team identity. These events were then searched for in the multi-tracked version of the session and if the recording was decipherable, relatively free from background noise and complete (i.e. not missing any turns because of distance from microphone), then it was transcribed. At the end of this process, from the three training sessions, lasting approximately four and a half hours (real time) in total, one hour's worth was transcribed giving 10578 words of transcription. The match data, by comparison had yielded one hour and ten minutes of transcribed data amounting to 13558 words. It should be noted however, that training session recordings frequently contained many short interactions, with long periods of noise caused by running. In addition, much of the speech captured on the player microphones consisted of short attention-getters (Ervin-Tripp 1976), used to signal readiness to receive a pass, such as yep, or here bro. Much of the time, this was all that featured on a recording while a training drill took place, accounting for a large part of 
the training recordings. Conversely, the targeted match day recordings were almost entirely transcribed, with only a few events containing too much background noise to decipher the full content of the interaction. A list of the events recorded can be found in Appendix C.

While not being as formulaic in structure as the match days, the training sessions also tended to follow a routine with some events that always happened and some that varied. Those that routinely occurred included the warm-up, during which the players performed simple passing routines supervised by Parky, who provided criticism and encouragement throughout. Another routine event was the team-run, which consisted of the players simulating a match without opposition and reacting to situations that were shouted at them by Tommo. During this Tommo and Parky watched and decided upon their selection of players to start that week's match. The last of the routine events was the de-brief, which took place only on the first of the week's two training sessions and was a meeting in the team room during which the team discussed what they could learn (usually what had gone wrong) from the previous match.

Van Dijk's (2008:115) definition of communicative events excludes spatial positioning as a part of discourse, categorising it as part of the context of an event. However I see the physical actions of the players and coaches, including features such as where they stood in relation to each other, as being a part of a communicative event itself. The spatial action of the players in forming a huddle, for example, plays as large a part in framing an event as belonging to the team and hence strengthens any identity work that is carried out in this space (a discussion of this is provided in Chapter 6). Additionally, the actions of the players in training session events are often a response to what the coaches say to them and what the coaches say is dependent on what the players do. Thus players' physical actions might be considered as responses to the spoken discourse of the coaches, which may then prompt further speech from a coach. Hence they are as much a part of the discourse of a communicative event as the speech because they qualify as non-verbal responses. In other words, rugby team discourse is not purely based on speech, it is constructed multimodally through the nexus of speech and action (Scollon and Scollon 2007). Aside from the repetition of these events week after week, what marked them out as discrete events was that they had names such as $D$-drill that had been assigned by the team (albeit derived from wider rugby 
terminology). Thus they were referred to by coaches when telling the players what they would be doing next, framing the activity as a specific discourse event and thus setting up the norm of behaviour for that event. By looking for events that had labels already assigned to them by the team, I was essentially following one of the fundamental tenets of a grounded theory approach of letting the data lead the analysis (Boeije 2010).

The less frequent, but nonetheless identifiable and internally named events, were scrum practice, lineout practice (which should both be self-explanatory) and shit-work, which was the team's name for fitness training. Notably the first two of these were discourse events that took place only within the Forwards while the Backs did not appear to have names for their activities, beyond code names for different plays that they would practice. A training session therefore would proceed according to the following pattern:

- Warm-up

- Shit-work (whole team) or split into Backs and Forwards

- Team Run

- De-brief (Tuesdays only)

One feature of every training session was that, as noted above, the team always spilt into the groups of Forwards and Backs for position specific training, with Tommo always training the Forwards and Parky always training the Backs. It was often not possible to stand close enough to the Backs to understand precisely what was going on for a lot of the time, so when the two groups split, I spent much more time observing the Forwards and thus have a much fuller picture of how they interact. However, the recordings of the Backs suggest that they do not interact as frequently as the Forwards, most likely due to the physical and spatial requirements of their positions. This is a topic that is explored in more detail in Chapter 6. In addition to the spatial differences in position, it was far easier for me to integrate with the Forwards than the Backs, because when I played rugby I was a forward ${ }^{15}$ and am thus much more familiar with the types of training they did than that of the Backs. Moreover, both the head coach and the senior captain were members of the Forwards and as leadership is one of the foci of this research I tried to observe them as often as possible. This is not to say that I avoided

\footnotetext{
15 Here, and elsewhere, I use "forward(s)" to refer to the generic field position throughout rugby, and "Forward(s)" to indicate the group of Forwards discussed here.
} 
the Backs, simply that there was far greater opportunity for observing and interacting with the Forwards and I thus gained greater insight into their practices than I did with the Backs. I attempted to fill this gap by questioning Parky, who coached the Backs, on anything that I did not understand about what the Backs did.

Despite the emphasis being on a physical activity, all these events involved a great deal of speech, mostly on the part of the coaches. This has been analysed across other sports as a specific coaching register (Heath and Langman 1994) and while the discourse features appear similar in form, for instance use of unmitigated directives mixed with compliments and criticism, previous research has not taken into account the detailed social context of the team culture which is analysable through ethnography, and which is key to understanding the function of coach discourse, as well as player interpretation.

In order to further understand the player interpretation of events, and to provide triangulation to the analysis of the focus discourse events, at several stages of the research feedback was given to the players and coaches. Their assessment of my analysis was used to further develop my understanding of the team and create new directions for the research. This is described in the following section.

\subsection{Interviews, feedback and follow-ups}

As stipulated earlier, one of the goals of this research, and of Linguistic Ethnography in general, is that the research participants are included in as many stages of the research process as possible (Cameron et al. 1992). In order to achieve this, the feedback of findings from the research to the participants took three forms: a written report for the coaches, interviews to discuss the contents of the report, and a workshop in which players were given a chance to analyse their own discourse and comment on it. Therefore it was not only one way transmission of information to the participants that was important, but also the feedback received from the participants about the results as this provided triangulation of my analysis. The research participants were thus able to influence the direction of the research at various stages. This valuable application of a grounded theory approach (Charmaz and Mitchell 2001) used the feedback of the participants as an early part of data analysis and thus determined not only what areas of the data to analyse, but also any gaps where further data collection was necessary. In this section the feedback methods and their contribution to the research are explained. 


\section{N. A. Wilson}

\subsubsection{Interviews}

Recorded interviews took place throughout the fieldwork, with varying degrees of formality. For the most part, however, they were informal and open-ended in their design, a fairly standard method of conducting ethnographic interviews (Schensul et al. 1999). They were what Wolfson (1976) describes as spontaneous interviews, in that I prepared some questions that would get the conversation started and then let the interviewee dictate the course of the interview, supplemented by questions that encouraged them to provide more detail if necessary. As I had the luxury of many potential informants and a relatively long period of time in which to do this I could afford to allow the interviewees complete control of the information they gave. In fact one of the coaches of the Colts started expounding his views on coaching philosophies to me at one session before I had even asked a question and I had to ask him to wait while I switched the recorder on. While this was useful in the initial stages of the fieldwork, more targeted, short interviews with quite specific goals became more useful for warranting events as good targets for recording, as the fieldwork progressed (Schensul et al. 1999; Silverman 2006).

The interviews also fulfilled the function of displaying my own understanding of the team to the interviewees, thus gaining further acceptance as well as triangulating my analysis of events. As described at the start of this chapter, the construction of the ethnographer's identity is a delicate operation and this was one of the ways in which this was achieved (Schensul et al. 1999). Although at first the interviews were a tool for gathering general information about the club and familiarising the team with the presence of a microphone, as well as a way for me to construct my own identity as an insider, as the season progressed, so did the role of the interview. More and more, the interviews became useful tools for triangulating my analysis of the data with the way the players and coaches might interpret it. It also allowed me to address questions regarding agency. This enabled a constant reappraisal not only of the way I interpreted the data, but also provided a warrant for where to focus my targeted recordings on match days and which players should wear microphones during training sessions. This is an example of how grounded theory methodology was present throughout this research. By always being open to new information and having a flexible approach to the way in which I carried out my fieldwork, the participants' feedback on my initial 
analyses led me to look at specific areas of discourse in the team that I may otherwise not have found.

The final way in which interviews were used was as a method of gaining feedback on the research experience from the coaches, Tommo in person, and Parky by email. These interviews were more formal in structure as I had specific questions to be addressed based on the report that had been provided for the coaches (see Section 3.5.2).

However, the formality of the interview structure was counter-acted by the relationship I had built up with the two coaches over the course of the fieldwork and thus I was able to ask very direct questions and receive detailed answers. It also gave the two coaches the opportunity to ask questions of me and to influence the direction of the next stage of data analysis.

\subsubsection{Reports}

After the data had been collected from three training sessions and four matches, but while the season was still in progress, a short break was taken from the fieldwork in order to take stock of what had been gathered so far and to run some preliminary analysis that went further than simply describing or transcribing the recordings. From this early analysis a report was written for the coaches to read which described some of the findings. This report exemplified how personal pronouns were used differently by the coaches and captains, and that there were four different leadership styles evident in the leadership discourse within the team.

The report was given to the coaches prior to the last match of the season and they were each interviewed about their responses to it. Tommo was interviewed in person, after the season finished, while Parky replied to my questions by email. Both provided some useful feedback about what aspects of team and leadership discourse they thought I should analyse. Both said that they would be interested in whether they used a more "directive or questioning approach", as Parky put it, and which was more effective. After reading the reports the coaches and I had an informal discussion on the team bus about who might be potential candidates for captain for the next season. Before my input, the coaches suggested all but one of the candidates that I identified as performing leadership behaviour. This provided some justification for my analysis of leadership behaviour within the team (Edge and Richards 1998; Nixon and Power 2007) and gave 


\section{N. A. Wilson}

me confidence that I had developed an accurate understanding of how the coaches interpreted player behaviour.

Thus the participants in this research not only assisted in the design of the data collection methodology, but were consulted in the analysis of the resulting data and were informed of results which they could use. In addition to doing research with the team, rather than research on the team, the approach taken here may be regarded as doing research with and for them, in that the results from the analysis were later fed back to the players by means of a workshop. In taking part in this final aspect of feedback they learnt about how they might use the analysis of their own discourse to develop their leadership skills.

\subsubsection{Workshops}

One season on from the fieldwork, and once the match data had been entirely transcribed and coded, I sought the players' perspective on some of the data. One of the goals of the research was that the players learn something from the season. I therefore gave them an opportunity to listen to and discuss an extract from one of the pre-match huddles and following this presented them with some of my own analyses.

Just as in the season in which the fieldwork took place, the players were split into groups during the weekly de-brief in order to work on a group discussion exercise set by the coaches. I used this to my advantage, not only by running the workshop during a de-brief, but by using the pre-existing groups to perform the discourse analysis task that I set them. However, these groups were not the same as the previous season, which split along positional lines. Instead the coaches had created the groups so that there was an equal spread of leaders among the groups. When asked why they had taken this approach, the coaches said that it was a decision influenced by the reports I had written during the previous season, in which I had identified that there appeared to be more examples of leadership among the Forwards than the Backs. That my research had been endorsed by the coaches in the way in which they organised the team, illustrates the potential benefit of the consultative approach taken in this research, as does the player reaction to the data, described here. 
Each player had a copy of the transcript of the chosen extract while they listened to the recording and each group was provided with a question sheet, with space for their answers. The questions were:
a. Who is the leader(s) in this recording? Why?
b. What do you think of this approach to leadership?
c. Could it have been improved? How?
d. Would it work for everyone? Why? Why not?
e. How could you use this?

The players reactions are detailed in Chapter 6, along with my own analysis of the extract they were played, but it proved to be a very useful method of triangulation (Charmaz and Mitchell 2001; Edge and Richards 1998; Lazaraton 2003; Silverman 2006). The main points that arose were that the players were aware of the need for a range of leadership styles, but were less aware of the role that context would play. I explained how they might adapt the leadership style they use depending on the context of the situation and they all agreed that would be an effective strategy. Overall I considered the player workshop to be a success and would have been willing to run more, however by this stage of the research I began to feel that, although the vast majority of players and staff at the club were still very welcoming, there were a few that were less happy about my return. They perhaps thought that I was taking up too much of their time, or that while I had earned membership of the previous season's team through my continual involvement, this was a different season and a different team. I therefore decided not to outstay my welcome and from then maintained looser links with the team. This of course raises some interesting questions about the temporally bounded nature of the team identity, which will be discussed in Chapters 6 and 7.

\subsection{Analysis}

Throughout this chapter reference has been made to doing "analysis" without specifying what this entails. When using Linguistic Ethnography as a methodology, although the process of conducting the ethnography is analysis, and data is examined in order to adapt the fieldwork approach, there is also a later more intensive stage of analysis. It is here that all of the knowledge gained through the process of ethnography, and recorded as field notes, can be used to interpret meaning in the interactions that have been 


\section{N. A. Wilson}

recorded. It is up to the researcher which form of discourse analysis to use at this stage (Rampton 2007a), or indeed to follow a different analytical framework entirely if appropriate. As my research questions are focused on leadership and team identity, I chose to use Interactional Sociolinguistics, an analytical framework that has been used extensively in analysing leadership discourse, particularly by the Language in the Workplace Project (Holmes 2005b; e.g. Holmes and Marra 2004a; Holmes et al. 2009; Vine et al. 2008). The strength of using Interactional Sociolinguistics is that, as described in the previous chapter, it focuses on a wide range of discourse features such as turn-taking, pronoun choice, swearing and humour and links these to stance and the construction of identity, as well as the pragmatic function of interactions. Interactional Sociolinguistics can only be used with detailed knowledge of the context of an interaction and hence it is often used in conjunction with ethnography as this is a productive way of gaining a deep understanding of the situation and social context of an interaction (Blommaert 2007; Hammersley 2007; Rampton 2007b).

In practical terms, what this meant for this research was tagging each transcript as I transcribed it with keywords relating to the functions, strategies and forms that were present in each. Essentially analysis comprised of matching up transcripts where there appeared to be a pattern in these keywords and looking at them line by line, and noting how each utterance could be interpreted in the light of my ethnographic knowledge. The keywords were also used to compare my data with other sociolinguistic research on leadership and team identity. The main tool that I used in doing this was a transcription and annotation package called Exmaralda, which allows transcriptions to be linked to sound files and multiple levels of annotation to be attached. It also provides a powerful search facility, so that when I found a particular form, I could search the entire corpus for it in order to see if it was used frequently for the same function. Although this is not a corpus-based study, I have used techniques from corpus linguistics such as concordancing and word frequency lists (Baker 2006) to support my interpretations of general discourse features within the team. In particular this was useful when investigating swearing as I was able to see who used swear words most, during which events and to what degree of intensity (Wilson 2009b). I was then able to analyse individual transcripts in detail with the knowledge that this discourse feature 
followed certain patterns in different events, thus enhancing the pragmatic interpretations I could draw from these events.

For the most part however, the process of analysis has predominantly relied on my understanding of the events that took place, using my field notes, to interpret the meaning of interactions and how these relate to leadership and identity through comparison with existing literature. These analyses have been warranted through the knowledge gained from early interviews of player and coach interpretations of events and triangulated using the feedback sessions and interviews described above.

\subsection{Summary}

This chapter has shown the way that reflexivity of ethnography and the adaptability of grounded theory underpins this research. Analysis and data collection are not truly separable using these approaches as the ethnographer performs analysis during data collection. In fact to call ethnographic fieldwork simply "data collection" falls far short of embodying the concept of Linguistic Ethnography. Although Linguistic Ethnography and grounded theory have been mentioned separately, they are to a certain degree coterminous as an ethnographic approach entails a grounded theory approach (grounded theory is the name for a methodology that is data rather than hypothesis driven). The methodology of this research is Linguistic Ethnography. What sets Linguistic Ethnography apart from other forms of ethnography is that it involves an analysis of language as its focus and thus necessitates recording authentic linguistic data. It is not however simply a way of gathering authentic data, but as Eckert points out it is "a process of mutual sense-making among all participants in the ethnography" (2000:76). Not only does the researcher gain a deep understanding of the social practices of a community, but the members of that community also learn about and perhaps change their practices through the self-examination brought on by ethnography.

As highlighted in the literature review, Linguistic Ethnography and the CofP model are closely linked as it is difficult to build a complete picture of the inner workings of a CofP without doing ethnography. Linguistic Ethnography is a methodology that allows the researcher to record authentic communicative events, and the strategies that form part of the repertoire of a CofP's negotiated practices. A detailed description of the CofPs that 


\section{N. A. Wilson}

were identified within the Prems is given in the next chapter, and the analysis of interaction in communicative events that is enabled by Linguistic Ethnography, provides a discussion of how CofPs form and are renegotiated by their members. 


\section{Chapter 4 Communities of Practice in the Rugby Club}

The concept of Communities of Practice, now widely used in sociolinguistics (Eckert and Wenger 2005; Holmes and Meyerhoff 1999; Meyerhoff 2001), is at its heart a model of situated learning through shared experience (Lave and Wenger 1991; Wenger 1998a). One of the reasons why it can be successfully applied to this rugby team is that the Prems, as a team occupying the level of rugby directly below professional rugby, may be considered a site for amateur rugby players to learn the practices that will make them suitable candidates for moving on to professional rugby. From interviewing players, I found that the majority of players in the Prems had a desire to play professional rugby. If the whole of New Zealand rugby is thus conceptualised as one CofP (disregarding for the moment the definitional requirement of mutual engagement), with the All Blacks as the core, embodying the legitimated practice that defines New Zealand rugby norms, then the players in the Prems can be seen as peripheral members on an inbound trajectory to professionalism. As they negotiate how one "does" being a rugby player in their local CofP (of the Prems), they develop their own practices and identity as a potential professional rugby player, which in turn keeps them on an inbound trajectory in the wider CofP of New Zealand rugby. In fact it is by becoming a core member of the Prems that a player moves inward on their trajectory towards professionalism. The practices that define the CofPs include the way they train, the way they play, but most importantly for this thesis, the discourse strategies that they employ in doing these.

The purpose of this chapter is to provide a description of the overlapping Communities of Practice that are embedded within the Prems, why they can be categorised as CofPs and how they develop as CofPs. The team itself is also analysed as a CofP, but one which draws its repertoire of interactional norms through a negotiation of the norms that define the embedded CofPs. Furthermore, I discuss how the team CofP forms first, before the other CofPs, because the composition of the team is under the control of the coaches. The analysis presented in this chapter builds on Wenger's concept of CofP boundaries and the nexus of multimembership (1998a:158) using the idea of layered simultaneity (Blommaert 2005) to show that leaders within the Prems do not simply 


\section{N. A. Wilson}

construct membership of different CofPs in different contexts, but simultaneously construct multimembership of CofPs in the team within the same interaction. Membership of the groups described here is both externally defined and internally negotiated, and although there is scope for members to redefine the boundaries of the CofPs, there are structural limitations to this that are inherent in the positional and hierarchical nature of the game of rugby (Melnick and Loy 1996).

The previous chapter discussed at some length the approach taken to fully engage with the Prems through ethnography and how this entailed becoming a peripheral member of the team. In addition to enabling the recording of authentic interactions within the rugby team, this has also given me a deeper understanding of the social practices that are used within the team to construct identity and leadership. While each of these will be examined in later chapters, this chapter examines how the team and the sub-groups within it can be analysed as Communities of Practice, thus providing a basis for a later discussion of the way in which team identity is constructed through practice. What defines the team CofP and the embedded CofPs are the repertoires of social and linguistic practices that they jointly negotiate over the course of the season (Wenger 1998a). Thus the negotiation of social and communicative practices results in a salient group identity for each CofP.

As discussed, the CofP is an analytical tool, not a social construct (Eckert and Wenger 2005). However it does reflect social reality in that the members appear to be aware of the fact they are members of a social group and that there are ways of signalling so, although how aware they are of this is not always clear. It is through close ethnographic study of the team in question that it has been possible to identify the groups in the team as CofPs and only in retrospect that the process of their development can be analysed. This should be borne in mind particularly in the section of this chapter that deals with how a CofP forms and evolves; it is not that a group has to go through a developmental process before becoming a CofP, but that having developed, CofPs can be analysed as having gone through this process.

As might be expected Wenger's three dimensions of Joint Enterprise, Mutual Engagement and Shared Repertoire (1998a) are vital in identifying the groups within the Prems as CofPs, but it is the last of these that is the most important for the discussion 
here, as Shared Repertoire is the observable (or recordable) linguistic product of CofPs. In this chapter, I demonstrate that each CofP develops a repertoire that is distinct from the other CofPs and it is this that defines it and gives it an identity that the members use in turn to define their own identities. The linguistic aspect of the repertoire that appears to develop differently in each CofP is the norms of politic behaviour (Holmes and Schnurr 2005; Locher and Watts 2005; Watts 2003). This will be the focus in this chapter and will be exemplified through a combination of ethnographic observations and extracts from the transcribed recordings made during fieldwork. There are of course other components of each CofPs shared repertoire that are identifiable in the members' discourse. However, it is often difficult to say whether these are practices that are part of the much wider community of imagination (Wenger 1998a:184) of rugby players. For instance, many of the players swear profusely, yet it is unlikely that this is a discursive strategy used in creating a unique identity as, for example, a Back playing in the Prems at this club. Rather it is a way of embodying the stereotype of hypermasculinity that exists within the imagined community of rugby players. That is not to say that the high incidence of swearing in the team does not also serve some other function; on the contrary it is a strategy used to engender solidarity as will be apparent in the discussion of how leadership is enacted within the team in the next chapter.

The first section of this chapter describes the organisation of the Prems and the four overlapping groups of members that can be classified as CofPs. The justification for this classification is provided through analysis of the interactions that take place in each CofP. The next section shows how one of these CofPs, the Forwards, follows Wenger's (1998b) stages of development in evolving from a community of imagination to a community of practice. Finally, the relationship between the leaders of the team and the CofPs is examined and this leads to the next chapter which deals with the leadership strategies that are evident in their discourse. This chapter however, is the opening of the discussion of the CofP framework as it relates to the Prems, not the whole. As discussed in Chapters 2 and 3, the CofP is what underpins this research and as such, communicative practice and the CofP construct are woven into each and every chapter of this thesis, and discussed in depth in Chapter 7. 


\section{N. A. Wilson}

\subsection{CofPs within CofPs}

The first groups of individuals that coalesce within the club are the three teams that comprise the senior section of the rugby club. These are the Colts (under 21s), the Senior Ones and the Prems. These three teams operate as a ranked hierarchy with the Prems (the focus of this research) representing the elite level of rugby at the club. The Prems, like the other teams, is a CofP on the basis that it fulfils Wenger's three dimensions of CofP categorisation, but also because it is clear that the Prems develop an identity as a group, and they do this through negotiated social practice.

As it contains multiple embedded CofPs, one might also analyse the Prems as a Constellation of Practice (Wenger 1998a). However, unlike a high school (Eckert 2000), a police force (Rock 2005), a call-centre (Brannan 2007), or an insurance company (Wenger 1998a), a rugby team is not merely an organisational context within which CofPs form. In rugby terms a better candidate for this would be the rugby club, within which there exist several teams, a committee (which one may view as a corporate structure) and a fan base, as well as all of the support staff that carry out the day to day business of cleaning the clubhouse, staffing the bar, cutting the grass and so on. Each of these can be analysed as a CofP, as can the teams, because they are groups of individuals who develop an identity based on what they do rather than who they are. They share a common understanding of what it means to be a member of their CofP, which is negotiated through their shared interaction. They also work together towards a shared goal and their membership of the CofP is constructed through their discourse. Within each of these CofPs there may be other CofPs, but just as discourse creates and indexes identity on many levels simultaneously (Blommaert 2005) a member of one may construct membership of multiple embedded CofPs simultaneously. As shown elsewhere in this chapter, this can be done through the same interaction, when the members of a CofP are all also members of a higher order CofP.

Unlike Eckert's (2000) Jocks and Burnouts, in the case of the rugby club, membership of these groups is not optional; among the players there is no equivalent of an inbetweener. This mode of belonging fits with Wenger's concept of a community of imagination (1998a:184) in that it reaches beyond direct engagement. For instance, in the rugby club there are groups of players that are determined by field position in the form of Forwards and Backs, and by club hierarchy in the form of coaches and support 
staff. However this is true of all rugby clubs and as a result stereotypical identities exist for each of these positional groupings. The same is true for the coaches and support staff. Thus there is a basis for group identity that pre-exists any that is created through the interactions of the members. What defines these groups as CofPs is the way in which they "do" being a forward or a back (or a coach) and the salience that this identity has within the team.

Also at work here is Wenger's other mode of belonging, alignment, which is important in analysing the identity of a CofP (1998a:186). This (as well as engagement) is what separates the Forwards in one team from the Forwards in another. For example, in this club there are three senior teams. However the Forwards CofP in the focus team, the Prems, has a distinct identity from the Forwards CofPs in the Senior Ones or the Colts because they are aligned with the other members of the Prems in terms of having shared goals created by the leaders of the team. At the same time all members of the club share an alignment in representing their locality and this is another factor that the Prems draw upon when constructing an identity for themselves.

The CofPs however, are not apparent from the beginning; they develop over time, as the players and coaches build up a shared history of interaction. At the outset of the season, there is only the team as a group of individuals who congregate around a mutual endeavour, and who only share practice insofar as it is generic practice held in common by all rugby players. Over the course of the season CofPs develop within the Prems through the practices that the players and coaches create in training sessions and matches. This is very much the lynchpin of the CofP model in that a CofP is something that develops over time, through interaction and negotiation (Wenger 1998a). Wenger (1998b) describes the evolution of a CofP in terms of five stages: Potential, Coalescing, Active, Dispersed and Memorable and his definitions are given in Table 4.1 below.

Table 4.1: Wenger's five stages of CofP development (reproduced from Wenger 1998a: 3)

Development Description

Stage

Potential
People face similar situations without the benefit of a shared
Typical Activities

Finding each other, discovering commonalities 


\section{N. A. Wilson}

practice

Coalescing

Active

Dispersed

Memorable
Members come together and recognize their potential
Exploring connectedness, defining joint enterprise, negotiating community

Engaging in joint activities, creating artefacts, adapting to changing circumstances, renewing interest, commitment, and relationships Staying in touch, communicating, holding reunions, calling for advice very intensely, but the community is still alive as a force and a centre of knowledge

The community is no longer Telling stories, preserving central, but people still artifacts, collecting memorabilia remember it as a significant part of their identities

The middle three of these stages (coalescing, active and dispersed) can be observed easily in the development of the Prems and the positional groupings within the team, with the coalescing stage occurring during the first few weeks of the season, when the Prems squad has been selected for the season and the players begin to train in their groups. The CofPs can be said to be active when they have developed practices through mutual negotiation that distinguish them as a group and hence construct their identity. This lasts until the end of the season, at which point due to the cessation of mutual engagement which occurs without training sessions or matches to attend, the CofPs become dispersed, still in contact through various off-season activities that the club operates but with a different focus and intensity of engagement.

The potential stage, arguably never exists for a rugby team. At this level of senior rugby all players have had experience of playing in several teams and as such have developed 
a shared (if not mutually negotiated) practice for joining a rugby team. This is aided by the generic practices that underpin rugby culture, such as a shared repertoire of rugby jargon, and discourses that are present in all rugby teams, such as the narrative of injury (Howe 2001). As mentioned in the previous chapter these generic practices aided my integration into the team just as they would a new player, and as such, the need to discover commonalities is, to a certain extent, overcome.

The memorable stage of the CofP is hard to analyse for a rugby team, as it requires some distance from the existence of the CofP in terms of time and contact with other members. Furthermore, the memorable stage can exist on a personal level as well as a group level, in the form of the impact that membership of the CofP has on its members' identities once the CofP comes to an end. They may use the practices developed though membership of the CofP either in constructing their own identity, for instance as exrugby players, or as input to a new CofP, such as the team that comes together the following year. Alternatively, for individuals like Jon, who left the club at the end of the season to play professional rugby, the Prems is a CofP in the memorable stage as he is no longer engaged with it, yet his past membership has an influence on his present rugby team practices and thus his identity as a rugby player. The memorable stage of a rugby team CofP may in fact become embodied by the clubhouse through the stories told there of past teams, the pictures on the walls and the trophies in the display cabinets.

Therefore, Wenger's model of CofP development is not fully compatible with the way in which a rugby team is reconstructed each year. Due to the cyclical nature of the seasons of rugby and the fact that many players will play for the Prems season after season, it can be conceptualised that rather than the linear model of CofP development that Wenger proposes, for CofPs like the rugby team, which are institutionally reified, there is a continuous model allowing one iteration of a CofP to build on the practices of the previous iteration. Thus the end of one CofP provides input, in terms of identity and negotiated practice, to the beginning of another CofP, showing the temporally dynamic nature of the CofP model. This argument is further taken up in Chapter 7.

As CofPs develop through interaction, the study of discourse within a group of developing CofPs is highly appropriate, and an ideal application of Linguistic 
Ethnography. Unlike previous ethnographic studies of CofPs (e.g. Bucholtz 1999; Eckert 2000; Owen-Pugh 2007; Vaughan 2007; Wenger 1998a; but c.f. Moore 2010a; King 2011), this research has followed the development of a configuration of nested, overlapping CofPs from start to finish, recording how they evolve from groups into CofPs and how this relates to the identity that they create for themselves in the process. Although the groups are defined at first by the structural and hierarchical nature of the game of rugby, it is the development of shared practices that defines these groups as CofPs, and this, as well as a description of the differences between the positional CofPs, is the focus of the next section.

\subsubsection{Field position as the basis of CofPs}

Once the three teams within the club have been selected, they train separately from one another, thus beginning the process of developing different practices. It is the coaches that determine these initial practices as they direct the players in their training. However, the players soon add to the shared practices of the group, introducing physical practices in terms of how they train, as well as linguistic practices in terms of how they communicate during training and matches. Although the focus of this thesis is linguistic practices, in the intensely physical and action oriented context of the rugby team one cannot analyse linguistic practice in isolation from physical practice as the two are mutually dependent (Goodwin 2000; Mondada 2009; Sobociński 2010).

Different ways of training begin to emerge as two distinct groups emerge: the Forwards and the Backs. The ways of communicating are influenced by differences in training such as player proximity and physical contact. Although these groups are defined by the positions that the players take up on the field of play (see Figure 4.1) and are thus not initially defined by practice, the way that each group trains is very different and this impacts upon the shared linguistic repertoire which they develop as they form a CofP. This can be compared with the way in which the claims processors in Wenger's (1998a) research are given tasks and goals and arranged in groups by the company for which they work, but develop their own practices. Doing this not only fulfils their work related goals, but develops a community identity, centred on the repertoire of practices they create.

One could therefore view the team and the groups within it as communities of purpose (Carotenuto et al. 1999; Schlager and Fusco 2004) which evolve into CofPs through the 
development of shared practice. In particular, the difference in the way in which the Forwards and Backs train is a reflection of the specific purpose of each group, and the different training practice both construct and are constructed by different discourse strategies. Once the Prems form out of the pool of players that make up the senior squad, their purpose moves beyond simply playing competitive rugby and can be looked at more specifically in terms of winning matches. The job of the Forwards is, put simply, to win the ball from the opposition. There are many tasks that comprise this enterprise but in the most basic terms this may be regarded as their prime function within the team. The Backs on the other hand, have the task of scoring, once the Forwards have won them the ball. Of course, how these tasks are carried out is another matter, but essentially if both the Forwards and Backs do their respective jobs more effectively than the opposing team, then (in theory) the team should win. The enterprises of the two CofPs are often explicitly specified in the pre-match team meetings as shown in Example 4.1, below.

Example 4.1: Team meeting

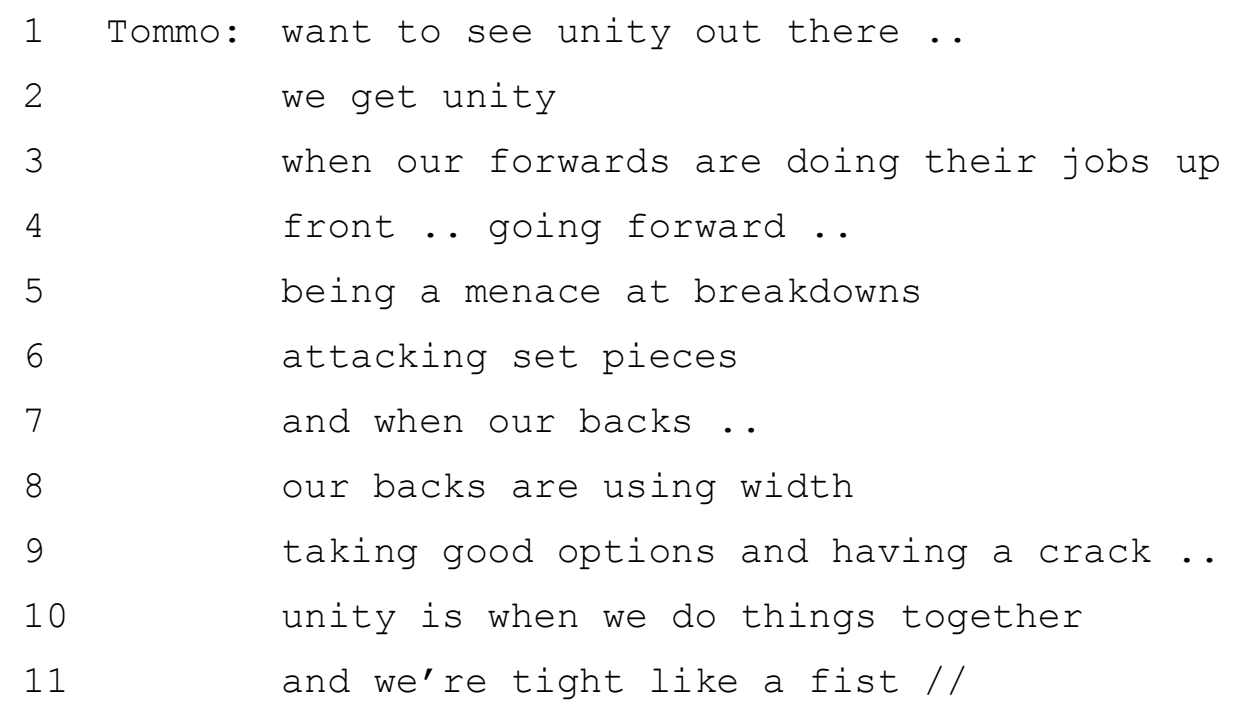

Tommo emphasises the difference between the Forwards' and Backs' jobs (lines 3-9) but also asserts the need for them to work together (line 10), thus locating each group within the team. By highlighting a difference in function between the CofPs, Tommo creates a differential aspect of identity which is incorporated into the way the two CofPs define themselves. However, it is position that is the starting point for the development of these two CofPs and it is important to understand how this is conceptualised in terms of the way that the players use the space of the rugby field. This is shown in Figure 4.1, 
which is a diagram of how the different positions are arranged during a scrum.

However, this pattern is also repeated at many other times in a match, such as at rucks and mauls. As discussed here and in Chapter 6, the use of space and the relative distance between players during training can be interpreted as having a major influence not just on the discourse strategies that are employed, but also the communicative norms that define each group (Mondada 2009; Sobociński 2010; Sullivan and Feltz 2003). This is because, simply put, the Forwards spend more time standing close enough to talk, and thus do so constantly, while the Backs are further away from each other and only talk in the intervals between moves (Melnick and Loy 1996). This is not to say that these physical and spatial factors are solely responsible for the different communicative norms which are negotiated in each group, but they provide a context within which some discursive strategies work better than others in fulfilling the transactional goals of each CofP. Thus the Forwards have far more opportunity to participate in phatic communication and solidarity building discourse than the Backs and furthermore, their constant physical contact with each other provides a degree of closeness and solidarity that it is less obvious in the Backs. The Backs also create solidarity; however they simply employ different strategies to do so and these may be seen as being determined in part by their on-field position (c.f. Holmes and Marra 2004b). These positions are demonstrated in graphical form in Figure 4.1.

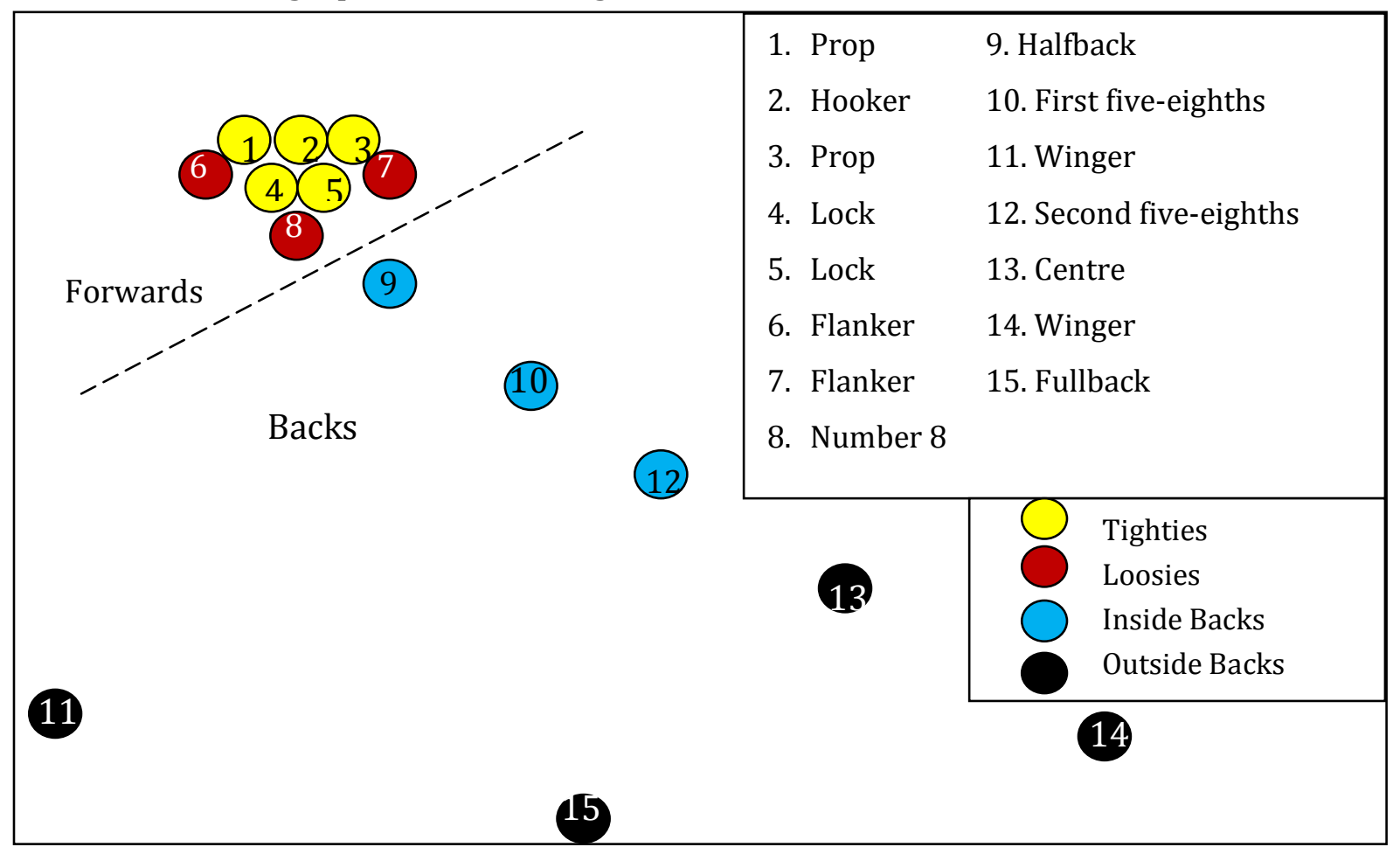

Figure 4.1: On-field positions and groupings 
As Figure 4.1 illustrates, other positional groupings exist within the two positional CofPs: Tighties, Loosies, Inside Backs and Outside Backs. Although these groups exist on a positional and organisational level, they do not appear to negotiate meaning or identity on a level that can be identified with membership of these small groups as opposed to membership of the Forwards or Backs. That the wider positional groupings of Forwards and Backs are renegotiated by the players as CofPs, while these are not, is perhaps down to the homogeneity of these groups within the larger positional CofPs. For instance, it is hard to see where the practices of Tighties and Loosies differ from each other in the way that differences exist between Forwards and Backs. However, while they may not be discrete CofPs from the Forwards or Backs, these are groups that are referred to in match day and training session discourse and each group carries with it a stereotypical identity that derives from the global repertoire of rugby ideology and from the in-game function of each position. These stereotypical positional functions are listed below.

- Tighties are the players that win the ball in scrums and lineouts; they are often described by Forwards as the players that do a lot of work in exchange for little glory. Phrases used by the Tighties and the coaches to describe the Tighties' role and thus construct their identity are: engine-room, grafters, hard yakka, it starts with us.

- Loosies are the opportunists of the forwards, they are faster than Tighties, and are the first line of defence as well as the first to react to any change in play. The phrases used by them and the coaches to describe their job are: first to the breakdown, steal the ball, no-one else can see the pattern, into everything that moves, shut them down.

- Inside Backs dictate the way the team play when they have possession, they can choose to run, pass or kick and this determines what the forwards have to do and what the outside backs do. Thus, they are in a powerful position. The phrases that are used to describe their role, by themselves and by the coaches, are: attack the ball, options, put them under pressure, dictate the patterns, we make the plays, run these guys off their feet, do the magic.

- Outside Backs are typically the finishers, the players who are fast and score the most tries. However, if the other groups are not performing their jobs, the 
Outside Backs will not get the ball. They are thus dependent on the Forwards and the Inside Backs to allow them to shine. They are also the last line of defence and thus can be blamed for tries being scored by the opposition. They seem to be referred to less frequently in the team discourse, but phrases used in reference to them are: using width, come into the line, sit back on the line, wingers scoring tries.

Although my initial knowledge of these stereotypes comes from my own rugby background, as shown by the phrases listed they are identities that are frequently indexed, and thus reconstructed in the discourse of the Prems (c.f. Ladegaard 2011a), often in the course of the pre-match build-up when stressing the importance of every player to the team. As such, the reference to these identities is a discourse strategy that enhances motivation, while also providing a way of addressing a specific group of players through defining group boundaries. It is thus a way of performing inspirational leadership, which will be discussed further in Chapter 5.

While they are groupings that are inherent in the positional organisation of rugby (Melnick and Loy 1996; Melnick and Thomson 1996), the four groups mentioned above are also used as an organisational structure in the Prems for cascading information. This however, does not appear to be enough to negotiate practices that construct local identity for these groups; rather they are sub-sections of the Forwards or Backs each contributing to the overall practices of those CofPs. However, one might ask how this is different to the way in which the Forwards and Backs contribute to the overall repertoire of practice that shapes the identity of the team. It is primarily because the communicative norms of these groups do not diverge from each other that I do not analyse them as CofPs.

While the Tighties, Loosies, Inside Backs and Outside Backs have been categorised as groups that are referred to through discourse, and have an identity created by description and indexicality rather than jointly negotiated practice, it is clear that the Forwards and Backs constitute different CofPs. They have fundamentally different repertoires in terms of both their discursive and physical practices. This is shown most clearly in the way that they train and the discursive norms that are constructed through this. The Forwards spend much of the time in close contact with each other, lifting and 
pushing each other on. Their norms of interaction are supportive and co-operative, using many compliments and encouraging phrases. The Backs on the other hand train (with Parky) to run pre-planned moves designed to penetrate the opposition defence. This means they must stand relatively far apart and when a move goes wrong it is easy to apportion blame to the player that made the mistake. On the other hand, in a match, each back has a far greater opportunity to achieve individual glory in the scoring of a try. However, rather than seeing the physical requirements of each CofP as determining their discursive strategies, I suggest that these are strategies that are negotiated in order to be effective in their position. It is essential for Forwards to co-operate, because one forward working alone cannot win the ball from the opposition (the Forwards' main job). However, there is less gain for the Backs in being as supportive as the Forwards, and thus more competitive norms may exist for them. As Example 4.2 illustrates, even in training the Backs spend time negotiating how to arrange positions for a practice run of a play and there is little agreement reached. At the beginning of the extract a discussion has been going on for some time about what they should be doing, with Will being the main speaker suggesting what to do. At this point however, we can see that the discussion is overwhelmed by jocular insults as well as disagreements. Will builds this into his attempt to steer the group into deciding what to do.

\section{Example 4.2: Backs at training}

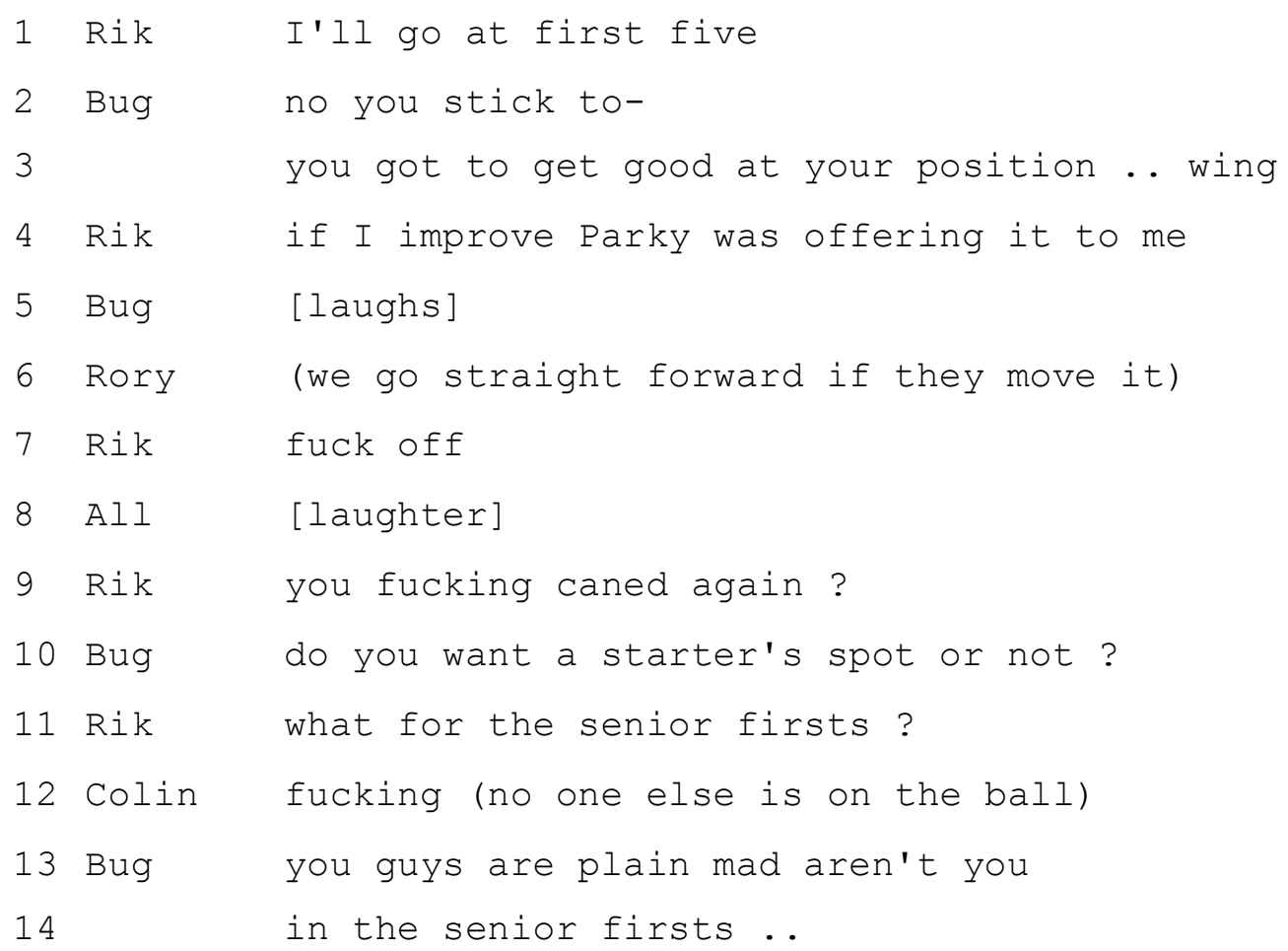




\section{N. A. Wilson}

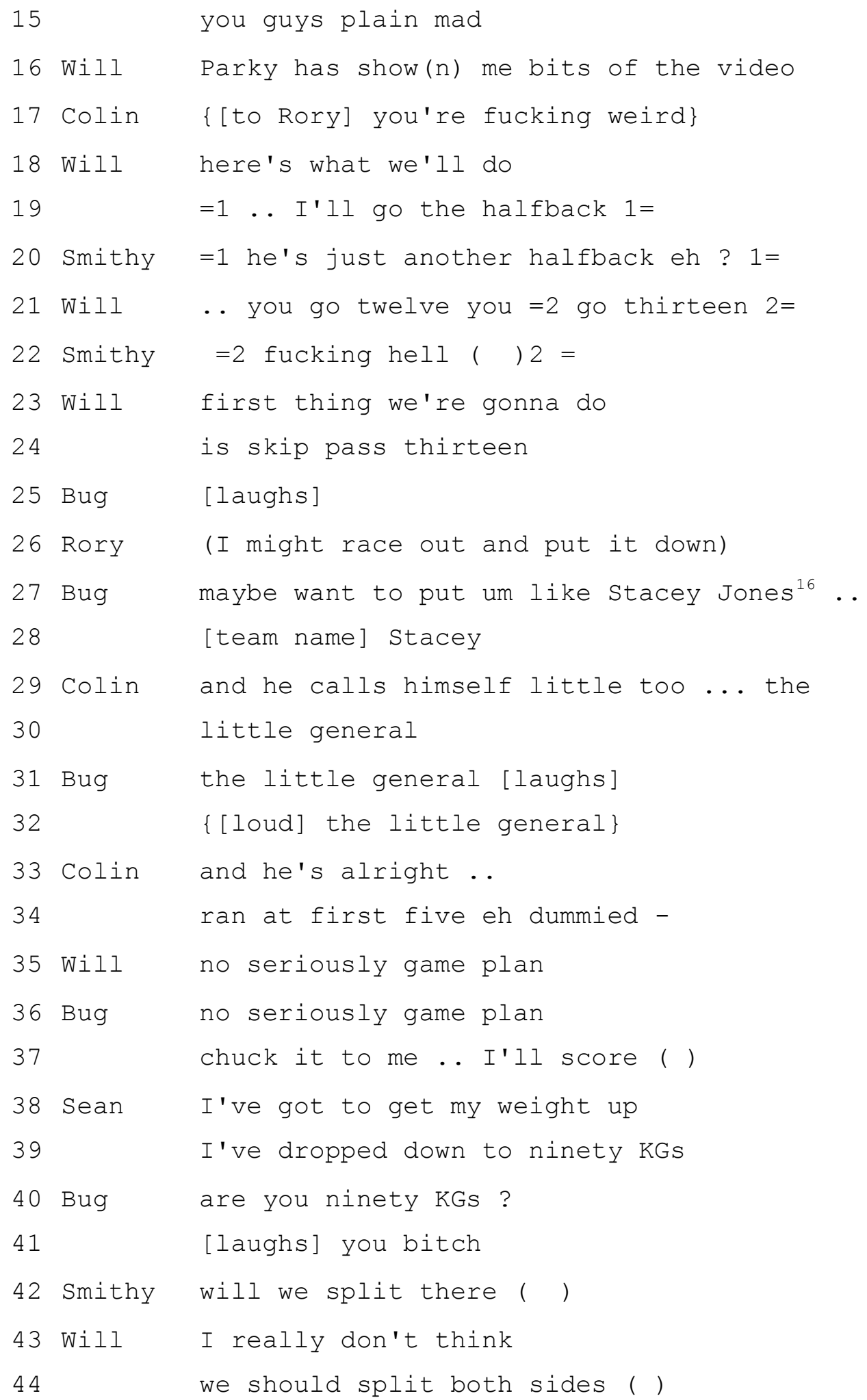

16 Stacey Jones is a former New Zealand rugby league player renowned for his small stature and his abilities as a playmaker, this has earned him the nickname "the little general". 
In this extract there are insults traded between many of the Backs that are involved (lines 7-15, 20, 29-32, 41). As demonstrated by the frequent laughter, the insults are jocular abuse (Hay 1994) and as such can serve to enhance solidarity (Daly et al. 2004; Kuiper 1991). This is a feature of the Backs' discourse that appears to have been negotiated as a shared practice, used to establish solidarity while also creating a competitive discourse structure. Furthermore, although the Backs seem to disagree about who should be doing what in this part of training, prior to this Will had been trying to take charge and lead them through a decision making process. He further attempts this in the extract by suggesting he has inside knowledge on tactics from watching the video analysis of the previous match with Parky (line 16), but he is interrupted by the end of the exchange between Colin, Bug and Rik who are jointly contesting Rory's suggestion the they go straight forward (line 6). Rory is a marginal member of the Backs' CofP as he has been playing for the Senior Ones until the previous match and although he knows the other Backs well enough through their mutual interaction in pre-season training, he has not been present for the negotiation of meaning in this CofP. As such, the other Backs seem to close ranks against him, putting down any suggestion he makes by suggesting that his membership of the Senior Ones means that he is on drugs (line 9), or mad (lines 13-15). This shows how insults can be used to define the boundaries of a CofP as well as to engender solidarity within.

Will's first comment on the game plan (lines 18-24) could also be interpreted as a continuation of the insults to Rory (who plays at number thirteen), suggesting that the first thing they do is miss him out (lines 23-24). Smithy however, sends an insult Will's way during his build up to this, referring to him as just another half-back (line20). This may be a challenge to Will's legitimacy in directing the play, or a reference to the fact that he has changed position during the course of the season, from centre to half-back. It seems that each of the Backs is attempting to contribute an insult, demonstrating that they are fully conversant with this discursive practice and thus can claim full membership of the group. Rory makes a further attempt to suggest a plan (line 26), which is this time met with a statement that could be interpreted as a sarcastic suggestion that Rory compares his skill level to that of rugby league player Stacey Jones and should thus be called [team name] Stacey (lines 27-34). Will attempts to stop the jovial nature of the discussion at this point and return to the formulation of a game plan. 


\section{N. A. Wilson}

However, Bug carries on with the humour suggesting that they should just give the ball to him and he will score (line 37). Notably, no-one challenges Bug in this assertion of his skill, unlike when Rory made a similar suggestion. This is likely to be because of Bug's status both as a core member of the Backs and as their official leader through his status as vice-captain of the Prems.

Despite the solidarity work that the jocular insults and humour create in this interaction, it is essentially a disagreement, and one in which no resolution is reached. Again, this is a typical pattern of interaction among the Backs when Parky is not present and Bug is. In other words, the presence of a particular leadership figure appears to have a significant impact on the contextual norms that are in operation. As Bug often adopts an authoritative, abrasive leadership style (as shown further in Chapter 5), he engenders competition and confrontation among the other Backs. However, when Parky is present he controls the structure of the discussion so that the different viewpoints reach an outcome that is mutually acceptable to all the Backs, thus creating a more conciliatory environment. Although few recordings took place in the Backs when neither was present, where it was captured, the Backs appear to adopt a more cooperative style of interaction, although the jocular insults are ever-present.

At this point, then, three features of the Backs discourse have been identified that can be considered part of their discursive repertoire: jocular insults (used to solidify boundaries and promote solidarity), a competitive style of interaction based on disagreement, and at times a more co-operative style. It seems however, that Bug, as the official leader of the Backs, has the most influence in creating the competitive discourse style. If we compare the discourse of the Backs with the Forwards, however, it is the competitive style that stands out as unusual and it may be that the overall team norm is more co-operative. Other research (Daly et al. 2004; Holmes and Marra 2002a) has shown that a contestive style of humour can serve to create solidarity in some groups, and this may be what is happening in the Backs. Moreover, in the unification of the core members of the Backs against Rory, the marginal member, humour is used as a means of marking a group boundary, highlighting Rory's lesser integration into the CofP (c.f. Holmes and Marra 2002b). 
Example 4.3, below, illustrates the differences between the communicative norms created by Backs and Forwards during training. While the Backs demonstrated competitive discursive norms, even when doing solidarity work through insults, the Forwards seem more overtly supportive of one another, with frequent compliments and encouragement rather than insults and one-upmanship. Example 4.3 took place during lineout practice, a key area for Forwards to practice and one which makes them stand close together and practice lifting one player (the jumper) while he attempts to catch the ball that has been thrown in by the hooker (Jon). Jon is in a key position in two respects. He takes the lead not only on the basis of his team captaincy, but also the leadership of this situation in which he starts with the ball and hence determines the play. This extract illustrates how supportive of each other the Forwards are and how directives are not questioned. Many of the Forwards contribute not only in terms of encouragement, but also in directing each other.

\section{Example 4.3: Lineout training}

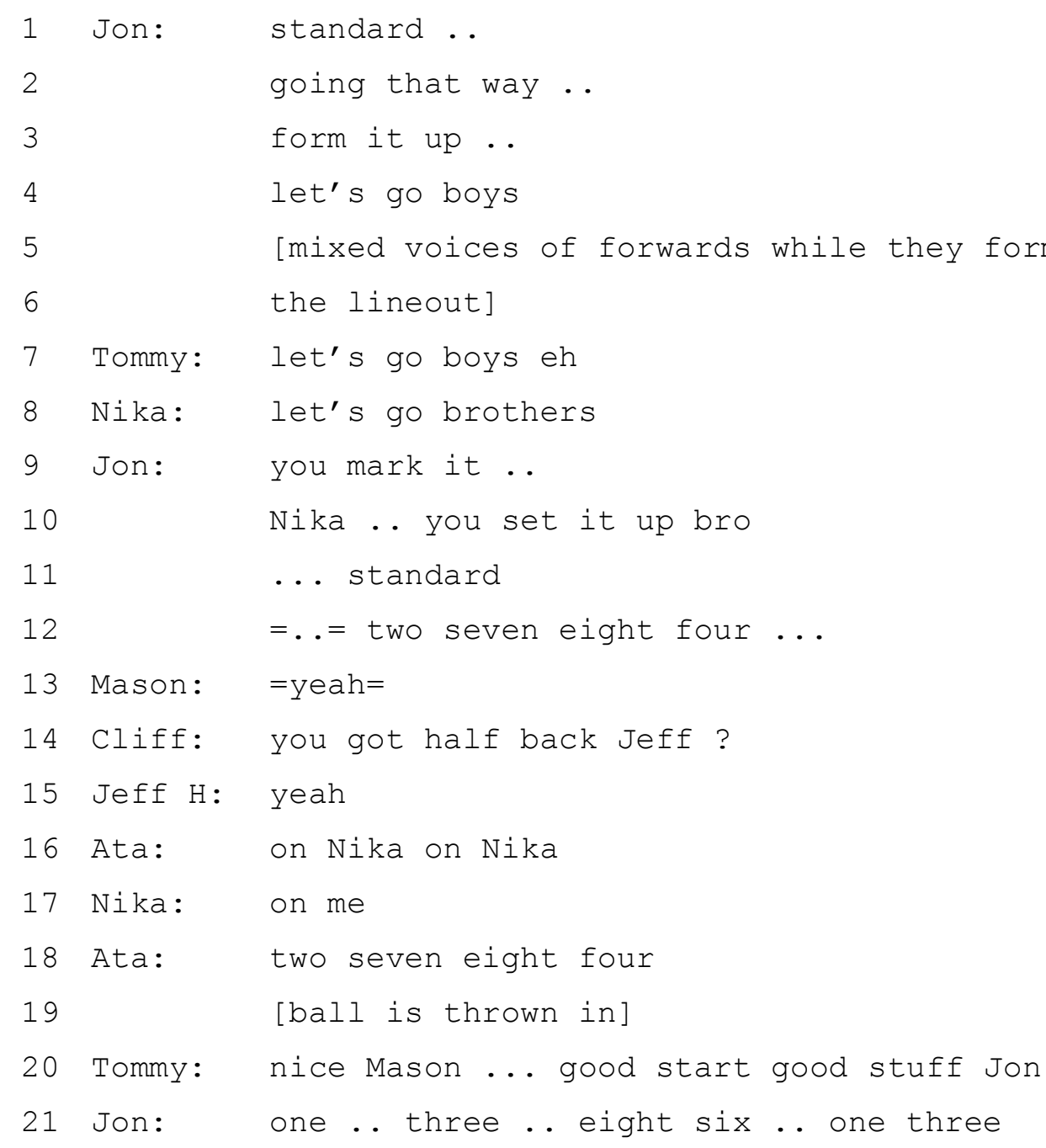$$
2
$$$$
3
$$$$
4
$$$$
5
$$$$
6
$$

13 Mason: =yeah=

14 Cliff: you got half back Jeff ?

15 Jeff $\mathrm{H}:$ yeah

16 Ata: on Nika on Nika

17 Nika: on me

18 Ata: two seven eight four

19 [ball is thrown in]

20 Tommy: nice Mason ... good start good stuff Jon

21 Jon: one .. three .. eight six .. one three$$
8
$$

\section{9}




\section{N. A. Wilson}

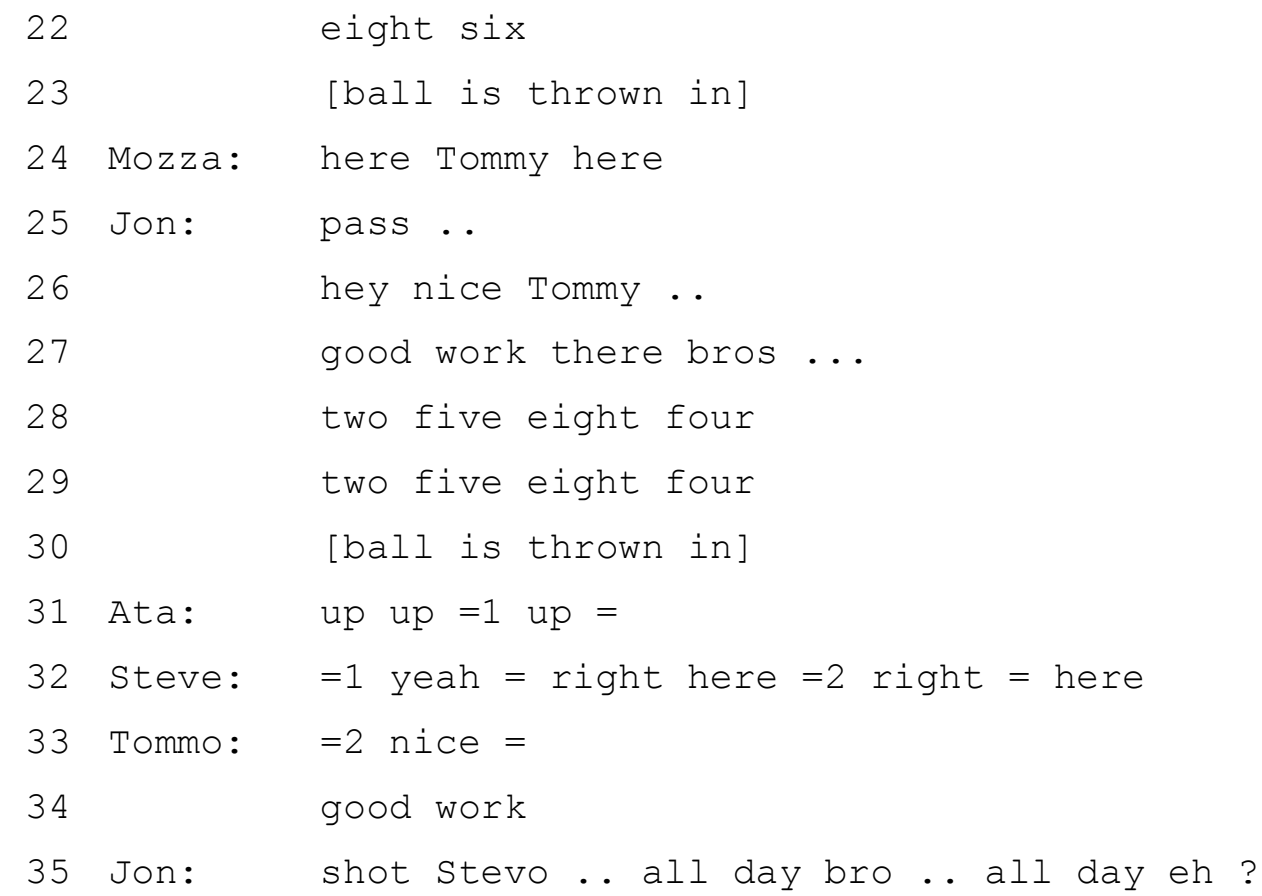

The constant communication between the Forwards seems to be important not only because it enables them to perform their roles effectively, but because it enhances their solidarity. What stands out in this regard is the repetition that occurs when a player makes a comment (lines $4,7 \& 8,16 \& 17$ ). Interestingly, on both occasions in this extract the player who repeats the comment changes the phrasing slightly. There is also however, a transactional reason for the repetition that occurs within the Forwards, not just when one speaker repeats another but when one repeats himself. Repetition could occur because the interaction takes place over a very short space of time, the players are moving about and there is often wind and other background noise. Therefore, important messages such as lineout codes, which communicate which player is to receive the ball (lines $12,18,2122,28,29)$ are always repeated in order to ensure that all have heard the call. This discursive practice may have become such an integral part of the Forwards' repertoire, for transactional reasons, that it is applied to other utterances, with a relational effect. For example when Tommy says let's go boys eh? (line 7) he is repeating Jon (line 4) but adding the pragmatic particle eh? This not only endorses Jon's original utterance which signalled to the Forwards that they were about to start, but this utterance can also be interpreted as encouragement (equivalent to come on), and the repetition helps to give it this meaning as well as Jon's original start signal. Tommy thus endorses Jon's leadership by repeating his instructions and builds on their function through his repetition. The plural address term, boys, is then modified 
in Nika's repetition to brothers (line 8). Not only does Nika's repetition of Tommy's words of encouragement endorse them and strengthen their illocutionary force, but his change from the already high-solidarity address term, boys, to the even more inclusive brothers, further enhances the message of solidarity and encouragement that Jon's original utterance conveyed. Thus, by repeating and strengthening encouragement, Nika and Tommy not only build solidarity among the Forwards, but between themselves and Jon as they all align to a common message. The use of repetition is an example of how the constraints, such as background noise, on a communicative event can lead to a practice being developed that not only overcomes the initial constraint but leads to the development of a relational practice that enhances solidarity through a similar strategy.

Nika again uses repetition in line 17, when he acknowledges Ata's advice to the other Forwards to bind round Nika (line 16). By repeating and rephrasing, Nika acknowledges this and reaffirms Ata's advice giving it validity and encouraging the other Forwards to follow it. As well as effectively endorsing Ata's position to issue advice, Nika's utterance also fulfils the function of an attention-getter (Ervin-Tripp 1976), by alerting the Forwards to his exact physical location so that they can be where they are supposed to be in the lineout. Thus, what appears at first as a simple piece of repetition functions on both a solidarity level and a transactional level.

Aside from repetition, there are also several examples of compliments in this extract (lines 20, 26, 27, 34, 35). These serve to construct the Forwards CofP as a highsolidarity, supportive community in which each player supports the other's efforts and applauds them for their successes. This was noted not only in lineout training but in every training drill involving the Forwards and it comes through in their pre-match huddles as well. In this regard the Forwards form a community of practice that has more overtly supportive discursive norms than the Backs, and is one of the communicative practices that distinguishes them as a CofP. Whether or not it is Jon's leadership that enables this is uncertain, but the frequent use of the address terms brothers and bros serves to emphasise a degree of collegiality that does not seem to be expressed among the Backs.

The Forwards further define their identity as a separate CofP to the Backs through friendly rivalry, thus showing how the competitive norm of the Backs is incorporated 


\section{N. A. Wilson}

into the communicative repertoire of the team CofP. This is played out in Example 4.4, which has been taken from a de-brief session that takes place at the end of the first training session of each week in the team room. This event serves to analyse what happened at the match on the Saturday and how the team can learn from this. The debrief involves Tommo asking the players to split into their positional sub-groups and come up with positives and work-ons from the game. Tommo then goes round the groups asking for what they have noted and writes the suggestions up on the whiteboard. They have already gone through the positives when the extract begins.

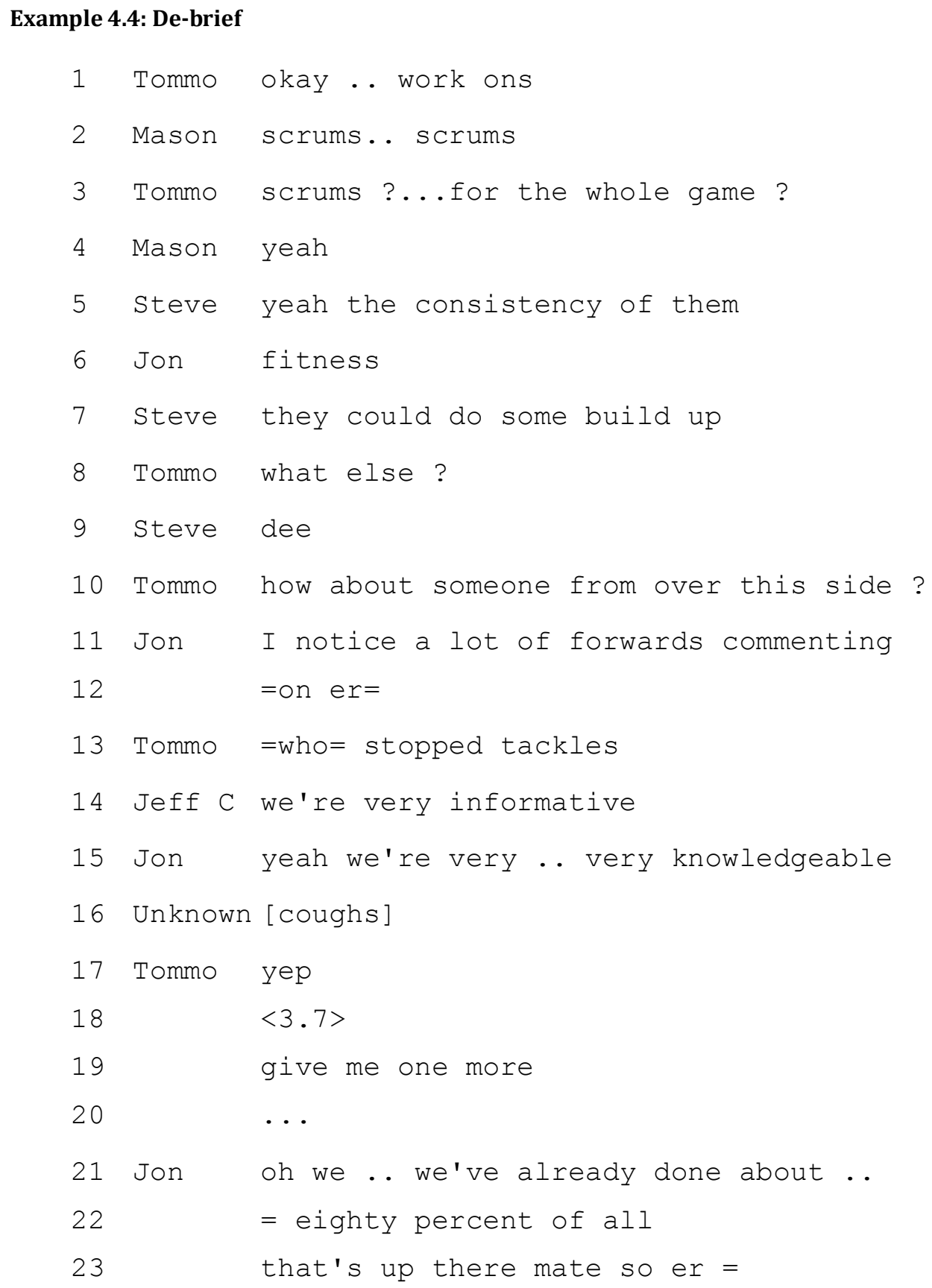




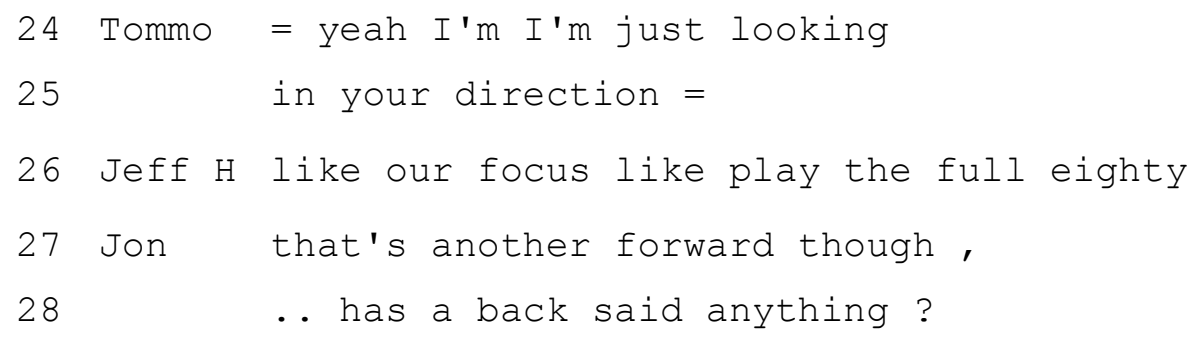

Jon jokingly protests that most, if not all, of the suggestions so far have come from Forwards (line 11)and uses this point to create an identity of the Forwards as hardworking and engaged when compared to the Backs (lines 15-28). That the Backs do not respond to this may provide an indication of Jon's unchallenged authority over all the other players. Not only does Jon lead in this jocular criticism of the Backs but he is supported by another forward, Jeff $\mathrm{C}$, who is a young member of the team and who is normally quite quiet. Jeff's support of Jon's stance is consistent with the supportive discourse strategy that is part of the Forwards' repertoire and indexes their identity as a CofP within the team. The Backs do not respond, either individually or as a group. This could also be analysed in terms of power in that Jon, as captain, holds institutional power, as does Tommo. Jon can thus challenge Tommo (and the Backs) with his complaint that the Forwards have already contributed enough (lines 21-22). If the hierarchy of the team is relevant, as would appear to be the case if analysing in terms of institutional power, then only Bug, as the vice-captain and self-titled captain of the backline may take a stand in opposition to Jon's assertion. That he does not perhaps demonstrates not only that Jon has authority over the whole team, as captain, but that given the supportive norms of the Forwards, he is always backed up by the other Forwards, whereas the Backs, given their more competitive discourse norms, may not present such a cohesive front. Tommo does not refute Jon's claim, in fact by explaining that he was not seeking further contributions from the Forwards (lines 24-25) he lends further support to Jon's point.

The one person in the team who is both a member of the Backs and in a position of greater institutional power than Jon is Parky. However in this situation, Tommo and Parky do not index their positional affiliations, but stand together as members of a separate CofP, the Leadership CofP. Through his shared leadership practices and mutual engagement with coaches in running the team, Jon is also a core member of this CofP, 
perhaps the reason that he is legitimised in challenging Tommo's authority in this situation. His complaint (lines 21-23) can thus be interpreted not as a player challenging the coach, but as a fellow member of the leadership team negotiating what is expected of the players. In his (tongue in cheek) complaint, he demonstrates his membership of the Forwards CofP, by making what could be interpreted as a challenge to the coaches on their behalf, while simultaneously indexing solidarity with Tommo by using the familiariser mate (Leech 1999; Rendle-Short 2009). Furthermore, in engaging in competition with the Backs in the first place, Jon is in fact subscribing to their norms of interaction, thus negotiating competitive discourse as a part of the team repertoire.

This is a demonstration of how multiple CofP memberships can be constructed in the same interaction and that members of each CofP could interpret the same interaction as following their (different) norms of interaction. This goes beyond the nexus of multimembership (Wenger 1998a:158), in that it shows the indexing of multiple identities through CofP membership to be not simply dynamic, but simultaneous and layered (Blommaert 2005:130). In other words, Jon is showing support for the Forwards (which they reciprocate), competiveness with the Backs and solidarity with the leaders. This raises the question of whether Jon, as a team leader, is a broker between the Forwards and Backs because he negotiates practice between the two. Wenger specifies that brokers exist on the periphery (1998a:108), but as may be apparent, Jon contributes so much communicative practice to the Forwards that he cannot be considered anything but a core member. Thus, rather than viewing leaders in the Prems as being brokers between CofPs, I analyse them as forming a CofP of their own, which overlaps the positional CofPs. Following other researchers of leadership (e.g. Crevani et al. 2010; Drath and Palus 1994; Drath et al. 2008; Raelin 2011), I analyse leadership within the Prems as a negotiable practice that creates meaning for the CofPs within the team out of which a team identity can be negotiated, rather than the imposition of a leader's wishes on a group of followers. This is not to say that the leaders of the team determine the discursive practices of the Team CofP, but that their legitimacy in representing the discursive identity of their CofPs is based on their core membership of these CofPs. Thus it may be seen that in the Prems, one has to be a core member of a CofP in order to legitimately "do leadership". 
Having introduced the idea in this section that leadership can be considered a practice around which a CofP can be identified, the next section will examine how the members of this CofP negotiate leadership discourse practices through mutual engagement and how the membership of the Leadership CofP can be described in terms of a member's trajectory towards the core of the Leadership CofP.

\subsection{The Leadership CofP}

Although the hierarchical structure of the rugby club and the teams within it position some individuals in official leadership positions (such as coach or captain), this is not the basis of the Leadership CofP that I have identified through analysis of the Prems' discourse. Rather, the Leadership CofP is a grouping of the individuals within the Prems who perform leader-like behaviour.

Typically, leaders in rugby teams have been players at some point, and through learning the practice of rugby leadership attain hierarchically recognised positions of authority, such as captain or coach. Thus their alignment towards the practice of leadership becomes institutionally reified (Wenger 1998a:261). Furthermore, the player leaders have to fulfil the same duties as the other players in addition to the work they perform in their leadership role.

Leadership can be regarded as a discourse practice in that it is constructed through discourse between leaders and followers (Bolden 2011; Uhl-Bien 2006; Vine et al. 2008). Furthermore, the members of the Leadership CofP in the Prems also share mutual engagement around a common enterprise and discuss strategies for achieving this, thus negotiating leadership practice and building a repertoire of leadership styles. The form of these leadership styles, and how they are used in complementary fashion is the focus of the next chapter; this section examines the membership of the Leadership CofP showing that leadership is a negotiable practice, and presenting the way the player leadership has been planned by the coaches, how this is interpreted by the players and what happens in practice.

\subsubsection{From plan to practice: The co-captaincy}

The coaches may be regarded as the founding members of the Leadership CofP, as it is they who initially determine leadership practice within the team. It is also up to them to choose the players who will be assigned leadership status within the official hierarchy 


\section{N. A. Wilson}

of the team (and club). As this section will show, however, selection as a leader is not the sole criterion for being a member of the Leadership CofP. Instead, it is performing leadership according to the negotiated shared practice of leadership determined by the CofP that defines a leader. Not all of the players selected as leaders are successful in enacting leadership and some players who are not selected as leaders perform as leaders, regardless of whether this status has been institutionally reified (Wenger 1998a). Example 4.5 shows the justification given by Parky as to the selection of the players who make up the team captaincy and how Parky envisages that it will work. The excerpt is taken from an interview conducted with Parky during pre-season training.

\section{Example 4.5: Interview with Parky}

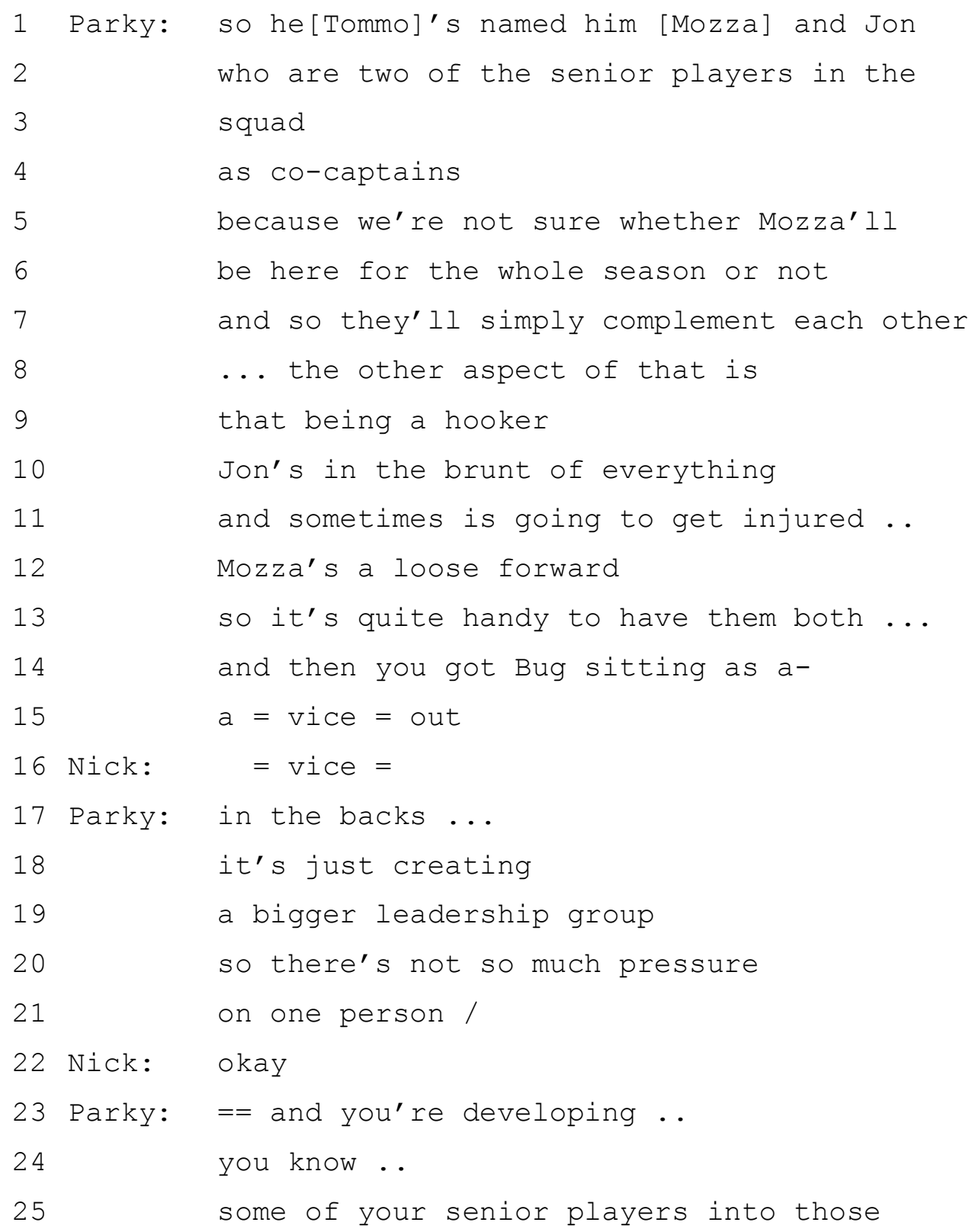




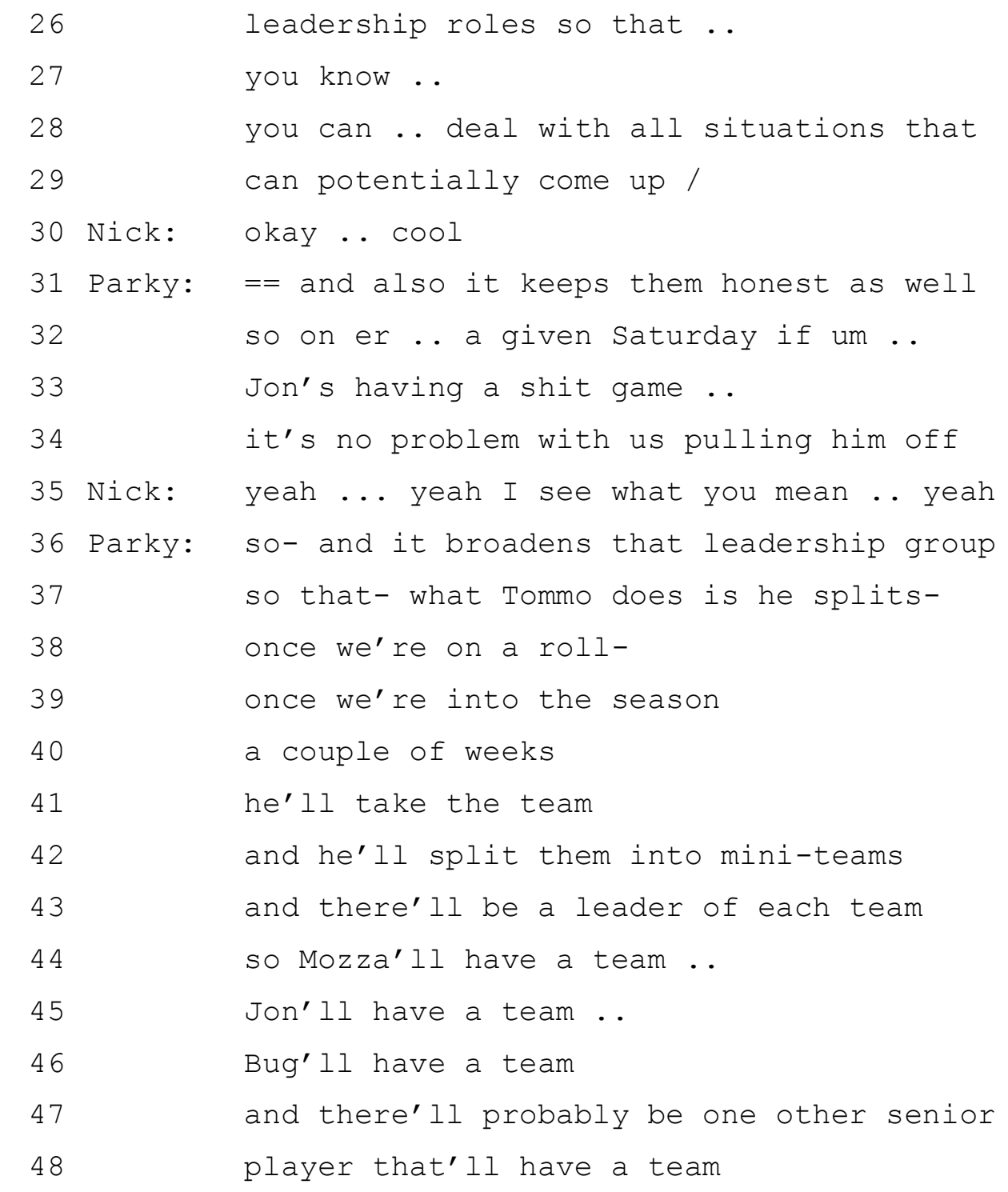

This indicates that there is a plan that the player leadership should function as a form of distributed leadership (Bolden 2011): a configuration of multiple leaders that encompasses co-leadership and shared leadership (Vine et al. 2008). This concept is explained further in Chapter 5. In outlining this plan, Parky names three players, Jon, Mozza and Bug, as leaders. Due to the hierarchical norms of the rugby club, it is an institutional necessity to name a captain (or captains) at the beginning of a season. The potential problem inherent in this is that prior to being made captain, the player has no opportunity to behave as a captain in a match situation, unless of course he was captain the previous season (which was not the case in the Prems). While they may have aligned their practices in such a way as to position them as potential leaders, it is up to the coaches to give their leadership institutional reification (Avolio, Walumbwa and Weber 2009; Davies 2005; Wenger 1998a). In other words, it is by speaking up at training 


\section{N. A. Wilson}

sessions, giving advice to other players and making themselves noticed that players negotiate the discursive practices that are used by the leaders. However, a player may be selected as an "official" leader, yet not successfully adopt the practices modelled by the core members of the Leadership CofP, the coaches, and hence never achieve full membership of the Leadership CofP, despite their "official" status as captains. By selecting two captains, therefore, it may be that not only are the coaches hedging against the possible injury of one of their captains (line 11), but against the possibility that one will not conform to the practice of leadership as it is constructed in the Prems. At this stage, Parky suggests an equal leadership status between Jon and Mozza. However, as is shown in the analysis of Jon and Mozza's leadership practice, these two players negotiate a somewhat different leadership role for themselves than intended by Parky, with Mozza enacting a role as Jon's deputy.

As the most senior figure in the player leadership, through the institutional reification granted him by the coaches and Mozza's complicity, Jon has a strong claim on core membership of the Leadership CofP. Through his alignment to the leadership practices determined by the coaches he achieves this and is thus in a position to renegotiate leadership practice, as discussed later in this section. The Leadership CofP not only contains institutionally reified leaders such as the coaches and captains, however. Other members of the team may align their communicative practices with the core members of this CofP in such a way that they may be considered leaders too. Figure 4.2 shows the players who I analyse as demonstrating leadership behaviour in the Prems and their positions relative to the core of the CofP. 


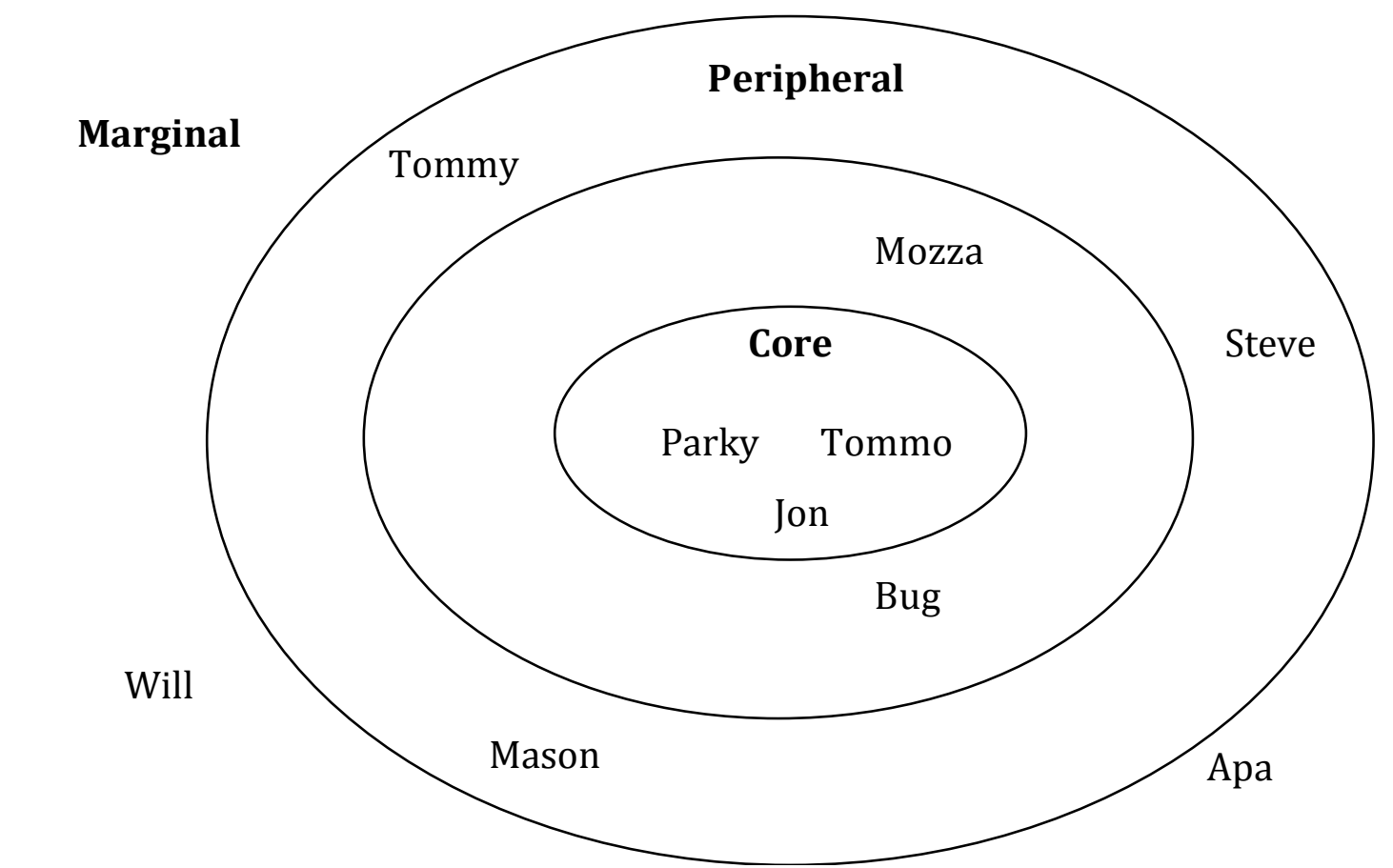

Figure 4.2: The Leadership CofP

In Example 4.5, Parky also outlines the intended structure of the team of being split into four groups. This is a reference to the aforementioned groups of Tighties, Loosies, Inside Backs and Outside Backs. As shown earlier in this chapter, the players did not seem to construct any meaning for these groups that set them apart from the larger positional CofPs. Perhaps as a result of this, the practice of using these groups as a way of doing leadership was not fully accepted by the players and although attempts were made to follow the coaches' plan, by mid-way through the season this practice had effectively been abandoned. This shows that although the coaches as the hierarchically defined leaders of the team, and, to begin with at least, the core members of the Leadership CofP specified a leadership practice for the team, this was negotiated not only by the players chosen to be leaders, but by the other players in their acceptance, or refusal of the legitimacy of these selections. This demonstrates the influence of followers in constructing leadership (c.f. Agho 2009; Crevani et al. 2010; Jackson and Guthey 2007).

The power of the players in co-constructing other players as leaders is shown in the way in which Mozza, while being a very good leader in terms of his decision-making ability, was never accepted as an inspirational hero leader in the same way as Tommo or Jon and thus could not successfully perform the practices that defined the role of captain within the Leadership CofP and the team. Instead he negotiated a position as a deputy, voicing support for Jon's decisions. However, this is not very different from the 


\section{N. A. Wilson}

behaviour that might be expected from him as a core member of the Forwards. In fact, in discourse terms, Mozza seemed to behave the same as other core members of the Forwards, fully subscribing to their supportive norms and using the compliment/encouragement strategy that has been noted as a feature of their discursive repertoire. Bug, on the other hand, negotiated a position as sole leader of the Backs by not only conforming to the inspirational leadership style of Jon and Tommo during huddles, but by also constructing a style of leadership that drew on his hierarchical status to exercise power over the other Backs. This seems to have been accepted by the Backs although whether, like Mozza, he was conforming to the discursive norms of the CofP or was in fact the driving force behind their construction is uncertain.

The descriptions of the leaders given thus far may suggest that the Leadership CofP is static. Membership of the Leadership CofP can also be viewed as a continuum between the core and periphery (Davies 2005; Wenger 1998a), with the members of it on different trajectories according to how closely their leadership discourse conforms to the shared practice that indicates core membership. If this model is used, then the Leadership CofP can thus be used not only as a way of grouping individuals around their construction of leadership discourse, but as a way of showing how leaders emerge and the process of moving from non-leader to leader. Becoming a member of the Leadership CofP can thus be viewed as the process of how players learn to become leaders, with their closeness to the core reflecting not only how much their discourse in matches and training sessions fits with the negotiated meaning of leadership discourse within the Prems, but the influence that they have in negotiating this meaning. The trajectories of the members of the Leadership CofP are plotted in Figure 4.3. 


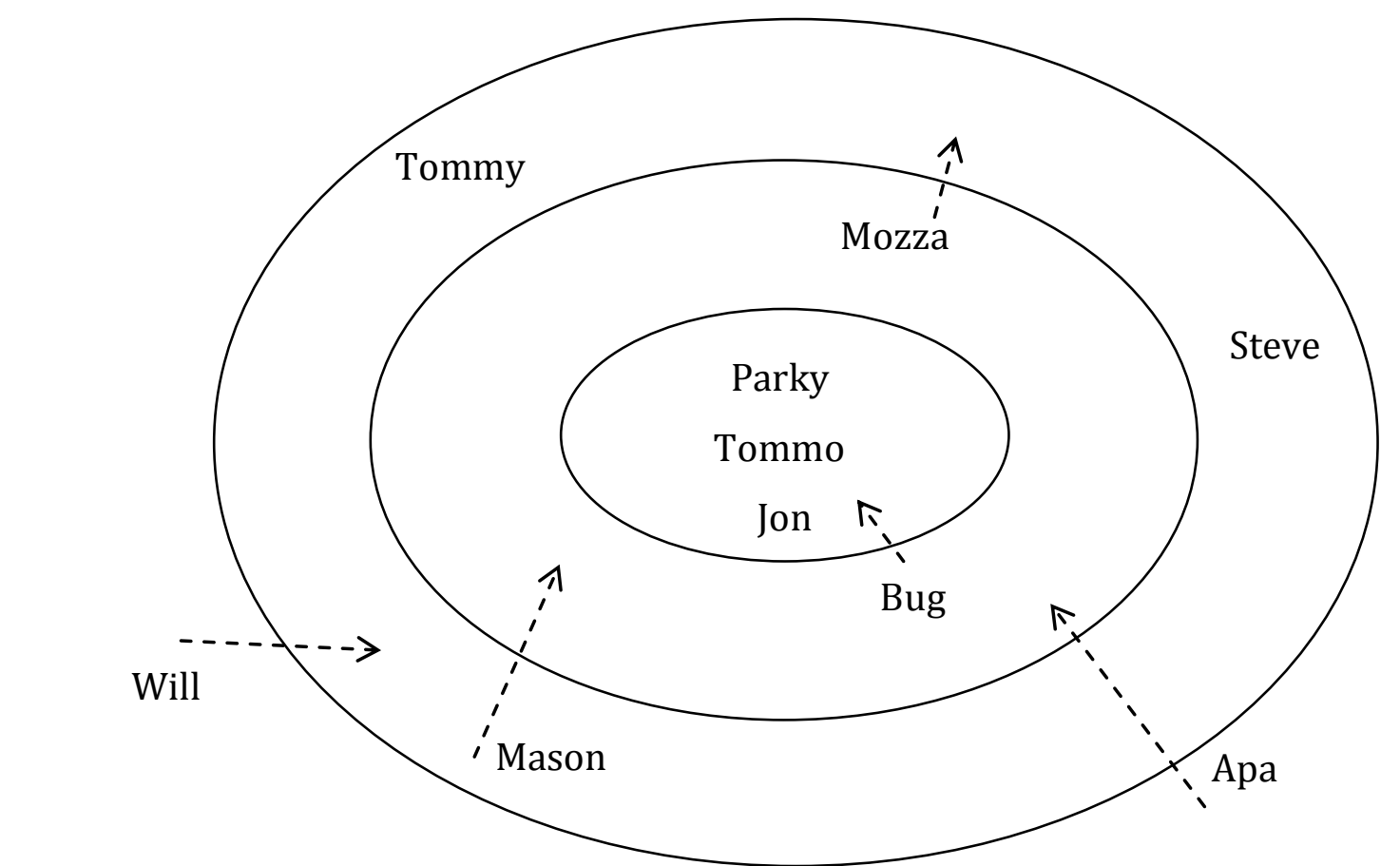

Figure 4.3: The Leadership CofP with trajectories

From this representation of the Leadership CofP it can be seen that the core is made up of Tommo, Parky and Jon. This is in part due to the institutional reification of their role within the team, which confers on them greater legitimacy in negotiating the discursive strategies that define leadership practice in the Prems. However, it also suggests that it is the leadership discourse that they produce that most influences the norms concerning what is considered effective leadership within the team. Thus, the players who are shown on the periphery perform some of these practices and through doing so align themselves with the core leaders. Others, like Bug perform some practices in line with core members, but also construct leadership strategies of their own. That these are accepted by the players legitimises these practices and thus sets them on an inward trajectory. Some players in the Leadership CofP create an inward trajectory through their increased performance of leadership discourse over time, such as issuing directives, giving advice, and complimenting other players on performance (this shows up in a broad analysis of the contribution of each speaker in the data). Others however, will use these discourse strategies but not perform any other leadership discourse, thus becoming perpetual peripheral members of the Leadership CofP. As has been discussed above, Mozza, despite being named as a co-captain at the start of the season did not perform leadership practice as it was constructed by the core members, and furthermore his stance as a leader was not fully accepted by the players until the end of 


\section{N. A. Wilson}

the season when Jon left the team and there was an opportunity to re-negotiate what it meant to be a leader in the Prems. Mozza's renegotiation of leadership practice is examined in detail in Chapter 7.

\subsubsection{Co-constructing leadership}

Although it has been mentioned that leadership practice is legitimated by followers (Drath and Palus 1994; Ladegaard 2011b), in viewing the leaders of the team as a CofP there is also a degree to which the leaders must be accepted as leaders by the other leaders. In order to show this, the interactional encounters between leaders need to be examined. In these, they not only make collaborative decisions that impact the way the team is led, but they also co-construct their relative positions in the membership of the Leadership CofP. Example 4.6 illustrates this using a conversation between Tommo and Jon in which Tommo seeks Jon's advice on which players to "promote" from the Senior Ones to the Prems.

\section{Example 4.6: Tommo and Jon discussing potential props}

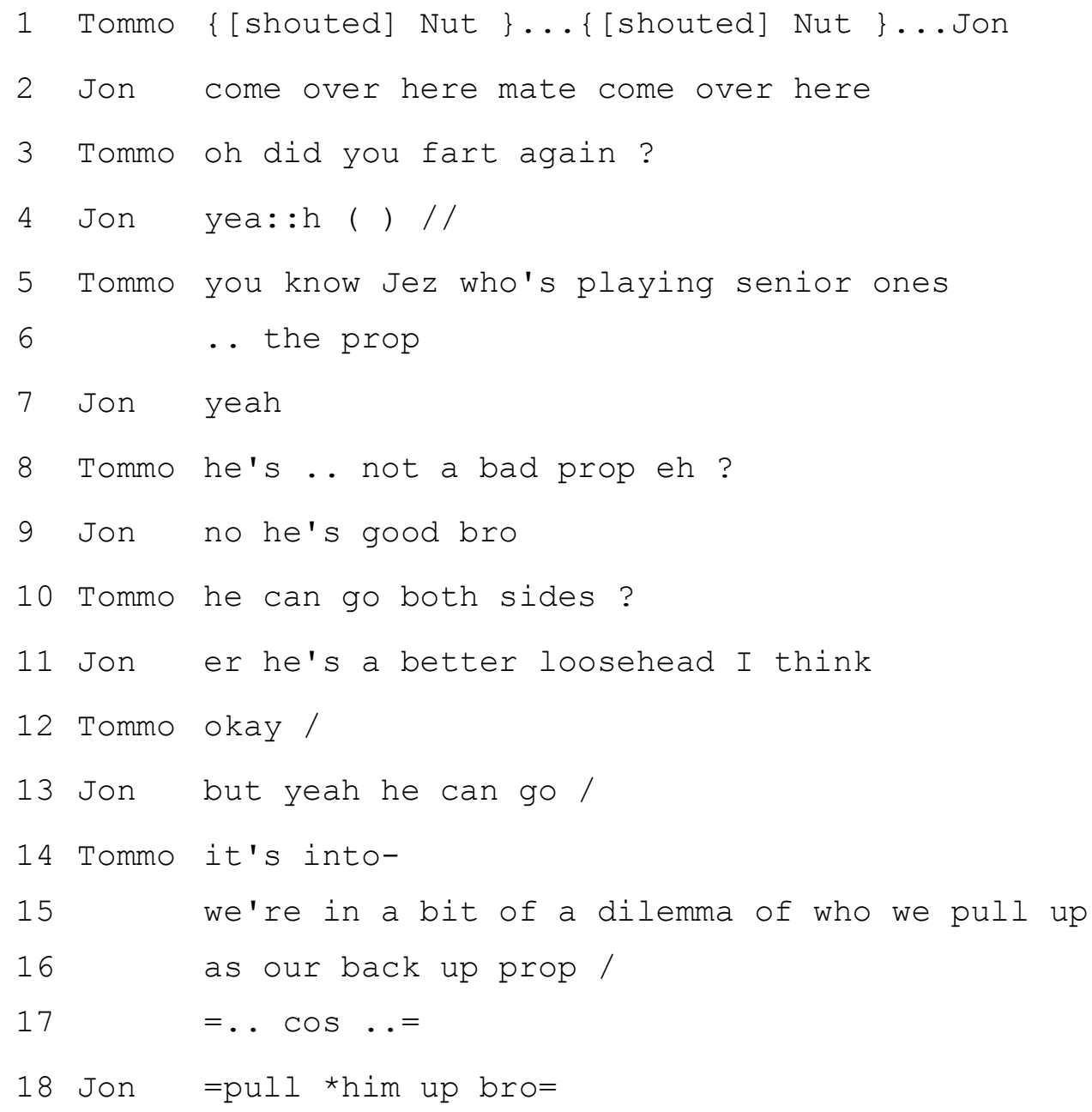




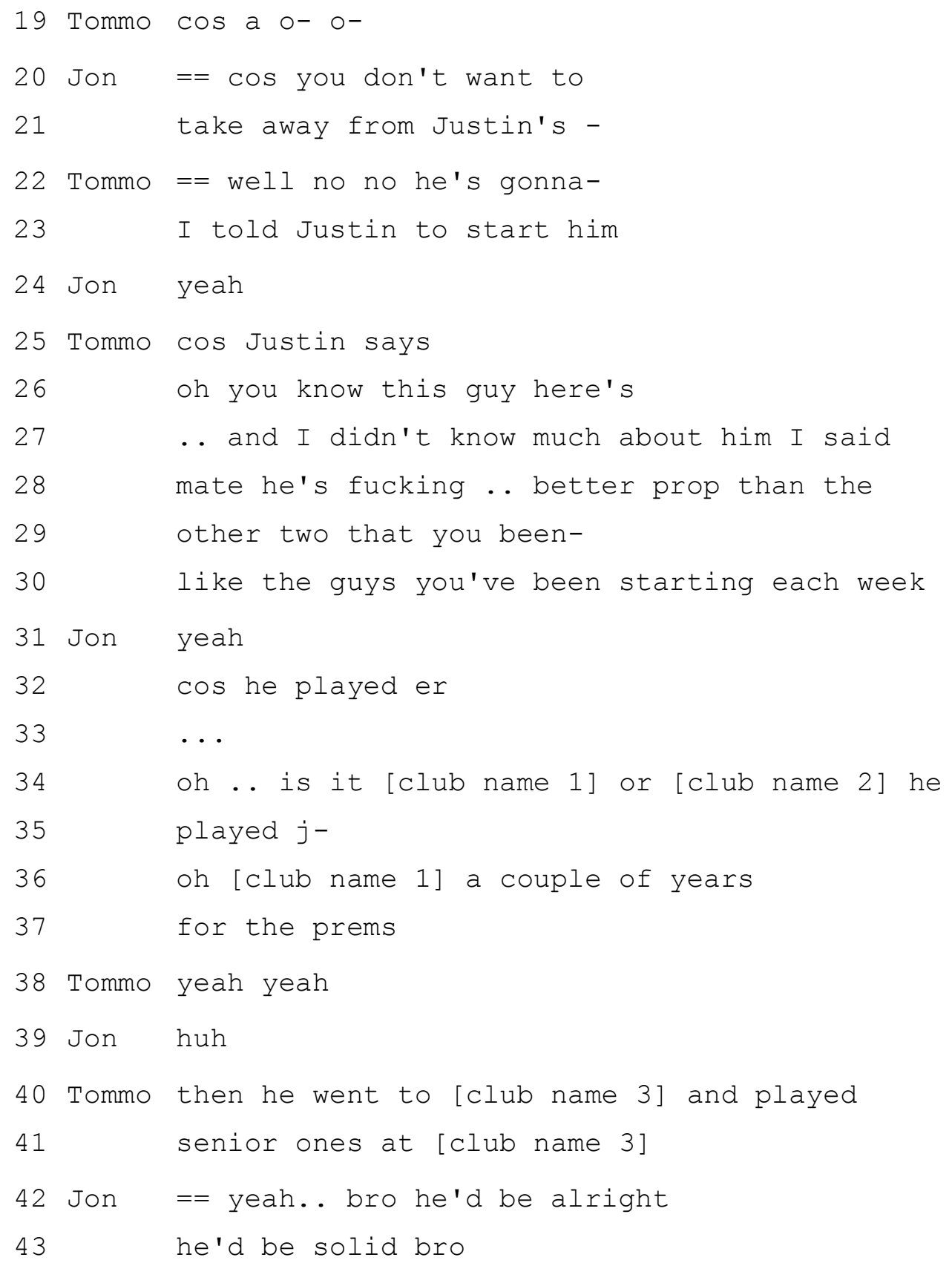

In the opening segments to this conversation, Tommo uses Jon's nickname, Nut, to attract his attention (line 1). This may be regarded as indicating to Jon that this conversation is between equals, rather than between coach and player. This is further indicated in Jon's use of the familiariser mate in his response (line 2) and elsewhere in the (lines 8, 17 and 40). Thus Jon and Tommo co-construct each other as fellow leaders. However, as is transpires through the course of the interaction, the transactional goal of Tommo's approach to Jon is to ask his advice on selection, a move which positions Jon in a greater position of power (Vine 2004). This further enhances Jon's status as a core 


\section{N. A. Wilson}

member of the Leadership CofP as being asked for advice by another leader would indicate that his opinion is valued and that his specific knowledge can contribute to the decision-making process of the team leadership.

The solidarity that is created in this conversation by the latching and many constructive overlaps, or "collaborative utterance construction" (Schegloff 2000:6), demonstrates that Jon and Tommo are happy to co-construct a solution to the problem at hand. The clearest example of this is when Jon completes Tommo's sentence for him (lines 20-21), interpreting the problem as Tommo being wary of intervening in Justin's (the Senior Ones coach) team selection. However, Tommo corrects this assumption (lines 22-23) by saying that he in fact told Justin to use the player in question, Jez. Tommo thereby demonstrates that he is not shy of exercising power over another coach. That he makes this explicit, while asking Jon for advice, strengthens their identity as members of the same team, through indexing the boundary between teams and the hierarchy that positions the Prems above the Senior Ones. This can be seen in the way Jon phrases Tommo's potential intervention in the player selection: pull him up (line 18). By referring to the movement between teams as up when referring to going from Senior Ones to Prems, Jon emphasises their higher status in the club, furthermore, by using the verb pull he suggests that Jez will do what he is told and move teams, emphasising Tommo's power as the Head Coach of the Prems.

This conversation between two members of the Leadership CofP shows them discussing leadership, in that they are talking about one of them (Tommo) exercising the power that he holds through his hierarchical status. By asking Jon's advice, Tommo positions him as an expert, and increases his status while promoting solidarity between them. By discussing leadership, the two co-construct each other as leaders and this demonstrates how members of the Leadership CofP are mutually engaged in the process of leadership discourse, as will be further shown in the next chapter. They discuss strategies for leadership and check possible outcomes of decisions with each other. This is one way in which leadership practice is negotiated in the team, among the leaders themselves. The other is with followers, the players, as leadership can only take place if the practice is accepted by the followers (c.f. Jackson and Guthey 2007). 
Therefore the negotiation of leadership is not just about negotiating a place in the Leadership CofP through demonstrating leadership behaviour. Once accepted, as Tommo demonstrates to Jon through seeking advice, a leader can negotiate how leadership might be done by suggesting possible strategies about which leaders should take on which aspects of leadership. The discussion of leadership strategies, in particular about how leadership is performed through communication, seems to be a distinguishing component of the Leadership CofPs communicative repertoire and one that defines it as a CofP. By discussing the leadership practice itself, the leaders not only construct themselves as leaders but define what leadership means to them and hence what constitutes the shared practice that defines membership of the CofP. In analysing which members of the Leadership CofP are core and which are peripheral it is perhaps this feature of their discourse that is most salient. While peripheral members seek access to the core through the alignment to the shared negotiated practices determined by the core members, once they are accepted into the core, they are legitimised in renegotiating that practice. During the course of the season this occurred most notably in the change in Bug's position in the Leadership CofP (as shown in Figure 4.3).

Although he started as vice-captain, and as has been shown adopted a competitive, confrontational leadership style, he was injured midway through the season and stayed in the Leadership CofP by renegotiating his position to one in which he assisted the coaches at training and matches, was involved in discussing team selection and tactics, and by doing so despite not playing, moved closer to the core of the Leadership CofP.

\subsection{Summary}

In this chapter I have argued that the CofP framework is useful for analysing the way in which leadership and identity are created through the discourse of the Prems. Analysis of the simultaneous multimembership of layered CofPs, suggests that rather than being brokers between CofPs, leadership itself can be considered a social practice and thus form the basis of a Leadership CofP. Furthermore, the Leadership CofP also represents the process by which leaders emerge and learn leadership behaviour, using the concept of CofP trajectories to show the path that the leaders take in becoming close enough to the core of leadership to renegotiate the leadership practices that define the CofP.

This chapter has also explored the idea that discourse in the rugby team should not be analysed in isolation from action and the use of space. It has been argued that two of the 
CofPs that can be found in the Prems, the Forwards and the Backs, are influenced in the communicative practices and discursive norms that they construct by the way in which their field position requires them to interact spatially. This should be not interpreted deterministically however. Rather, the physical practice of each CofP creates an environment in which the development of, for example, the supportive norms of interaction of the Forwards are beneficial to the CofP's performance as Forwards; by working together in their discourse, they promote a culture of co-operation that is important in their successful performance as Forwards. The Backs, on the other hand, seem to construct more competitive norms of interaction, which both index and perpetuate an ideology of the individual back as a star player.

In summary, this chapter has attempted to further develop the CofP model as used in sociolinguistics, by viewing it not just in relation to communicative practice, but by including physical practices in the analysis of a CofPs repertoire of negotiated shared behaviour. Furthermore, the analysis has identified a system of layered CofPs as dynamic groupings, using ethnographically acquired insights to describe how they move from communities of purpose within the imagined community of rugby, to communities of practice that construct a salient group identity through their shared communicative practices. The next chapter focuses on the discursive repertoire of the last of the CofPs described in this chapter, the Leadership CofP. Having demonstrated why the group of leaders within the Prems should be considered a CofP, the different leadership strategies evident in its members' discourse are examined, as are the processes which describe best the negotiation of leadership practice: distributed, shared and co-leadership. 


\section{Chapter 5 Leadership}

Leadership has been 'in' for so long I can hardly remember when it was 'out'.

(Rost 1993:7)

... there are almost as many definitions of leadership as there are persons who have attempted to define the concept

(Bass 1990:11)

The problem with studying leadership, as the above quotations illustrate, is that despite being a topic that has been studied across many disciplines for at least a century, there is no one coherent account of leadership. Nonetheless, in recent years some attempts have been made to achieve an integrated definition of leadership within the burgeoning field of leadership research (e.g. Vroom and Jago 2007; Winston and Patterson 2006). However, the only aspect of leadership that appears to be universally agreed upon is that a leader is a person who influences people to modify their behaviour in order to comply with the wishes of the leader (Rost 1993; Vroom and Jago 2007). This deals with the what but not the how.

The aim of this chapter is to detail exactly how leadership is 'done' in the Prems. The previous chapter was concerned with the CofP model, and introduced the idea that within the Prems there exists among the many embedded CofPs, one which is defined by the practice of leadership. This chapter will build on this by providing examples of the different leadership strategies that make up the repertoire of the Leadership CofP and the forms of language that are used to perform them. This takes as a basic assumption that leadership is a practice, in other words something that is "done", rather than an inherent personality trait or quality as it has often been viewed by scholars of leadership psychology (e.g. Avolio et al. 2009; Bono and Judge 2004; Campbell, Simpson, Stewart and Manning 2003; Groves 2005). As might be expected from the theoretical frameworks within which this research is located, I view leadership as a socially constructed process in which leaders and followers each play a part and which is 
achieved through a variety of discourse strategies (Drath and Palus 1994; Fletcher and Kaufer 2003; Holmes, Schnurr, Chan and Chiles 2003; Komives et al. 2005; Ladegaard 2011b; Uhl-Bien 2006). This follows much of the existing research on leadership discourse which has shown effective leaders to perform a range of leadership strategies, attending to both transactional (goal-oriented) and relational (people-oriented) needs (e.g. Fletcher and Kaufer 2003; Holmes and Schnurr 2005; Holmes et al. 2003, 2009; Schnurr 2008b; Schnurr and Chan 2009). Extending the discussion begun in the previous chapter, I will demonstrate how the leaders in the Prems use their multimembership of the team's CofPs in order to achieve both co-leadership (Sally 2002; Vine et al. 2008; Wilson 2009a) and shared leadership (Fletcher and Kaufer 2003; O’Toole et al. 2002; Pearce and Conger 2003).

Prior to this analysis however, a brief summary of the existing research in the field of leadership discourse will be examined, focusing in particular on unpacking the concepts of shared and co-leadership as well as the notion that a leader's identity is coconstructed by their followers as well as the other leaders.

\subsubsection{Defining leadership}

As noted above, many scholars have defined leadership. However, many of these definitions arise from vastly different epistemological backgrounds. There are definitions of leadership in psychology that portray it as (the result of) a collection of inherent traits such as charisma and extroversion (e.g. Bono and Judge 2004; Campbell et al. 2003; Gordon 2007). There are definitions originating in organisational and management studies, which are primarily concerned not just with how to do leadership, but how to do it well. Many of these definitions link leadership with management and structural hierarchy (e.g. Alvesson and Sveningsson 2003; Fairhurst 2008; Ling 2008; Svennevig 2008). Although a growing number of management and organisational scholars see leadership as a co-constructed process between leaders and followers (e.g. Komives et al. 2006; Ling 2008; Lord and Brown 2004; Morgeson et al. 2010; Svennevig 2008; Winston and Patterson 2006), most continue to approach leadership from a prescriptive rather than a descriptive stance.

The study of leadership discourse differs from the psychological and the business-led approaches to leadership in that it does not look for the "right" way to do leadership. 
Rather, leadership is seen as being made up of many, contextually dependent discourse strategies. Some of these may overlap; some leaders may use many strategies and others use few. In essence, however, research on the discourse of leadership views leadership as accomplishing not just transactional, or task-based, goals, through influencing others, but also maintaining harmony within a team or CofP through attending to the relational needs of the group (Holmes et al. 2003). The relational component of leadership that Holmes et al. (2003) espouse is demonstrated in the rugby team most clearly by the amount of work the leaders put into building a team identity and inspiring the team to perform to the best of their abilities, but is also evident in the way they employ humour, narrative and jocular insults in their discourse with players. However we can also take a more sophisticated perspective on leadership performance in the Prems. In fact, performing leadership through the rhetoric addressed to the whole team prior to matches may be considered both relational and transactional, because it not only solidifies the team as a group but may also help result in a good outcome for the club in the form of a victory in the match. Thus, just as with other forms of discourse, leadership can operate on multiple levels simultaneously.

In addition to achieving both relational and transactional goals, the same piece of leadership discourse may be interpreted by different players in different ways. What one sees as threatening, another might perceive as goal-setting. As the transactional outcome of leadership discourse is shown by the effect that it has on followers' actions then it is clear that the followers play an important part in the construction of successful leadership through the way they match their understanding of the leaders' discourse to what was intended. Followers signal their understanding and acceptance of a leader's stance through their actions and their discursive participation in the process of giving meaning to leadership. It is for this reason that leadership discourse has to be appropriate for the CofPs within which it operates, as leadership discourse, like all other interaction which occurs, operates according to the interactional norms which are negotiated as part of the shared practice of a CofP.

In business-led leadership research, transactional and relational leadership have often been cast as oppositional gendered leadership styles where men are thought to tend toward task-based leadership while women are more likely to perform relational leadership (Eagly and Johannesen-Schmidt 2001). However, research on leadership 
discourse (e.g. Holmes 2005b, 2008; Ladegaard 2011b; Mullany 2007) has shown that this is not the case. Rather, a combination of styles is used by the majority of effective leaders. An effective leader, it seems, adapts their leadership style dependent on contextual variables. Thus the leadership styles that are observable within a group are not created by the leader alone, but in concert with the group that is led (Ladegaard 2011b; Uhl-Bien 2006). This does not mean that there is no element of strategy or agency to a leader's choice of leadership style, as examples in this chapter illustrate. There is often a high degree of pre-planning involved in the particular approach taken by a leader, especially in the case of co-leadership (Fletcher and Kaufer 2003; Vine et al. 2008). The conclusion to be drawn from this is that leaders do not exist in isolation; a leader cannot be a leader without followers and it is the interaction between the leader and followers that creates leadership.

Leadership in sports is sometimes equated with coaching, a style that is also employed outside of sport, for instance in business leadership (e.g. Gordon 2007; Holmes 2005b; Holmes et al. 2003). Coaching is a register that is saliently identified in sports discourse (Heath and Langman 1994; Kuiper and Lodge 2004) with distinct features such as repetition and formulaic imperatives that are shared with non-competitive sports instruction, for example, an aerobics class (Delin 2001; Kuiper and Lodge 2004). However, the role of the coach in a rugby team is not just to improve players' rugby playing technique and fitness through instruction. It is also the job of the coaches (and captains) to inspire the players to perform to their best on match days. The coaches must therefore balance the roles of instructor and inspirational leader in order to fulfil their duties as coach. Furthermore, they must also take care of management tasks such as player selection and discipline. Each of these facets of the coach's job requires different discourse strategies and together they contribute to the construction of the coaches as leaders. However, it is the players' acceptance that confirms this leadership identity, with the way in which values and practices that the coaches communicate to the players being incorporated into the identity that they forge.

\subsubsection{Multi-membership and distributed leadership}

Building upon the previous chapter which argued that the leaders themselves form a CofP within the Prems CofP, this chapter contends that multiple CofP membership can be used as a resource for building leadership strategy. Through membership of the 
Leadership CofP, players and coaches may jointly construct their identity as leaders within the team, while simultaneously maintaining membership with other CofPs. Hence, two of the main practices that the Leadership CofP is defined by are shared leadership (Fletcher and Kaufer 2003; 0’Toole et al. 2002; Pearce and Conger 2003; Schnurr et al. 2007) and co-leadership (Sally 2002; Vine et al. 2008; Wilson 2009a). Simply put, shared leadership is where two (or more) leaders of close or equivalent hierarchical status divide the responsibilities of leadership between them, with both taking an equal level of responsibility. In the Prems the first definition describes the relationship between Tommo and Parky. Co-leadership on the other hand, describes the function of deputy leaders and the work that they do in carrying out tasks (often relational) that contribute to a leader's effectiveness. A more detailed view of shared leadership (as described by Fletcher and Kaufer 2003) incorporates this, describing it as a collection of practices that are distributed across all levels of an organisation, with different functions of leadership taking place at each level. In the Prems, this seems to be the relationship between the coaches and Jon, and between Jon and the other players who act as leaders.

In other words, the simple view of shared leadership is a flat model of leadership while the complex one, and co-leadership, are ways of building co-operative leadership strategies across a hierarchy. These jointly produced strategies, which are constructed through the discourse of the leaders and their followers, can be collectively viewed as distributed leadership (Bolden 2011; Crevani et al. 2010). They are not only a way of effectively carrying out leadership through the division of leadership duties (playing to individual communicative strengths), but are also about how the leaders communicate with each other and in particular, how they strategise their leadership through backstage conversations (Goffman 1959; Richards 2006, 2010). Thus the Leadership CofP has a particular form of mutual engagement, in the form of these conversations, where the leaders not only make shared leadership decisions but also negotiate their own identities as leaders and members of the Leadership CofP. Having negotiated their leadership identity in-group, they may then perform leadership more effectively with the team as a whole. This chapter will explore some of the back-stage conversations in which the leaders construct their identity within a distributed leadership structure then 


\section{N. A. Wilson}

demonstrate how the leaders use this in the front-stage interactions they have with the players in their role as leaders.

\subsubsection{The dynamism of leadership styles and types}

There appears to be a tendency in much of the existing leadership research to categorise leaders into types, or to pinpoint specific leadership styles. While it is recognised by researchers who take a social constructionist view of leadership that features composing these styles index stereotypes held about different types of leader (Ladegaard 2011a; Mullany 2010), a large amount of the leadership literature originates from management and organisational studies, where there is a strong tendency to specify different leadership styles: authoritative, transformational, transactional, and so on (Goleman 2000; Mandell and Pherwani 2003). Specific leadership styles can certainly be found to be used by leaders and categorised as, for instance, authoritative. However, a leadership style cannot be categorised simply by examining the discourse features that it uses, and extending this to other leaders as a generally applicable way of communicating leadership. Instead, leadership styles should be thought of in terms of identity because they are highly specific to the communicative competency of the leader, the social-cultural and negotiated behavioural norms of the community in which the leader operates, and transactional and relational needs of the organisation (Baxter 2010; Holmes and Marra 2004a; Ladegaard 2011b). Needless to say, each situation in which a leader "does" leadership is likely to require a new leadership style or, at least a re-tuning of an existing one. Whether or not a leader adapts their leadership style to the situation may be regarded as a marker of their efficacy as a leader (Holmes 2005b).

Essentially, there are a number of linguistic features that have been identified as effective strategies for doing leadership. The particular combinations of these features may be identified as leadership styles. However, many of the same features could be used in a context with different norms of behaviour and the leadership identity that results could be entirely different. This is because of the importance that followers play in leadership. It is their interpretation of how the leader presents him or herself as a leader that is a key factor in the effectiveness of the leadership, as ultimately leadership can only be considered effective if the followers follow (Agho 2009; Lord and Brown 2004; Vine et al. 2008). In other words, the way in which a follower frames a leadership stance is crucial in constructing effective leadership. Using this concept, the 
construction of leadership identity can be readily compared with the construction of social identity, as described in Chapter 2.

Having made a case for not defining hard and fast leadership styles and types, it must also be said that certain archetypal (and often stereotypical) leadership styles are useful as reference points when discussing leadership, if only for something with which to compare authentic data. Furthermore, the existence of leadership stereotypes can be regarded as existing at the ideological level of leadership identity that is indexed through the use of particular leadership strategies. For instance, when looking at how leadership is enacted differently (or not) by men and women, stereotypes of male leaders as being goal-oriented and female leaders as more people-focused are often referred to (e.g. Holmes 2005b; Holmes and Schnurr 2005; Ladegaard 2011b). However a sizeable amount of research has shown that these stereotypes do not reflect reality. While there are certainly male leaders out there that do not pay much attention to the relational needs of their followers, there are also many who emphasise solidarity as a core part of their leadership and draw on a range of discursive resources such as humour and small talk to achieve this (Daly et al. 2004; Holmes and Marra 2004a; Mirivel and Tracy 2005; Richards 2006; Schnurr and Chan 2009; Schnurr et al. 2007). Likewise, female leaders have been shown to construct a leadership identity that indexes stereotypes of male leaders in order to successfully exercise power in organisations, especially those that are male dominated (Baxter 2010; Holmes and Marra 2004a; Holmes and Schnurr 2005). Neither male nor female leaders however, are confined to one leadership style, or indeed one leadership identity. Their leadership identity is shaped by what works for them in the organisation that they lead. Because they are likely to find themselves in a variety of different situations, the feature of leadership discourse that has been found to be most important above all others is the ability to change leadership styles to match the context (Holmes et al. 2003, 2009; Ladegaard 2011b; Uhl-Bien 2006).

As the Prems are an entirely male group, it may be useful to be aware of certain male leader archetypes that have been identified in other organisations (Holmes 2009). This is not to say that we can pigeon-hole the leaders in Prems into one or other of these, but that certain leadership styles that they create may index these leadership archetypes. The first of these leadership types is the Heroic Leader (Jackson and Parry 2001). This is 
a style based upon the great man concept of leadership (Bass 1990; Rost 1993), and relies upon the idea that it is through his exploits that a male leader is judged a good leader. Although this kind of leader is critiqued heavily in much of the literature surrounding leadership and gender (e.g. Binns 2008; Grint 2010; Krantz 1990; Uhl-Bien 2006), it is certainly a stereotype that persists and this was evident in several of the answers that the players of the Prems gave when asked what they thought made a good leader. The repeated answer was that they lead by example. In other words, by displaying good skills and commitment on the rugby field, players like Jon, Mozza and Bug would inspire others to the same level of achievement. The heroic leader is authoritative, decisive, inspirational and charismatic (Holmes 2009; Marra, Vine and Holmes 2008) and certainly some of the leaders in the Prems exhibit these types of behaviour at times.

The father-figure leader uses a paternalistic mentoring leadership style, paying close attention to the relational needs of his followers but maintaining authority. In the Prems, a leader who regularly indexes this identity is Parky. He is often involved in mentoring players one-on-one, yet when it comes time to mete out punishment to players who have not performed as well as they could, Parky also tends to be the leader who orchestrates this. The father leader might be described as firm but fair. In terms of discourse features, the paternalistic leader tends to give advice, criticise when things are wrong but compliment when deserved (Holmes 2009; Marra et al. 2008).

Another leader identity is that of the mate or good bloke, especially in the egalitarian culture of New Zealand (Holmes 2009; Marra et al. 2008). This is a form of leadership that emphasises solidarity and is thus highly relational (Uhl-Bien 2006; Vine et al. 2008). There is also a high degree of democracy in this form of leadership. Given the high level of solidarity that exists within the Prems, this leadership type is often constructed when the coaches are working with the players in training sessions.

These three leadership types are not an exhaustive description of the way in which male leaders can be categorised and one could certainly not say a particular leader is, for instance, merely a heroic leader (c.f. Mullany 2010). However, he may index that leadership type through relating a narrative of his exploits in overcoming great odds. On another occasion he may be mentoring and thus construct himself as the father figure, 
giving sage advice based on years of experience, but retaining a sense of distance from the mentee. On the other hand the leadership identity that he creates may not index any of these types and in fact be based entirely on the leaders' experience within the group or CofP in which they operate. Furthermore, these leadership types do not encompass the way in which deputies can construct their leadership identities. For some it may be as a trusty lieutenant, effectively facilitating their leader's instructions. Alternatively a deputy may construct themselves as ambitious by challenging and negotiating leadership decisions. A deputy may construct an identity as an apprentice leader, ready to learn from the more experienced leader in order to develop their own skills and progress up the leadership hierarchy. All of these can be found in the discourse of the Prems, as will be shown in the course of this chapter. The point to remember is that while such styles or identities can be labelled in analysis, they are dynamic and are (re)created every time they are constructed in discourse, adapting to the needs of the leader and followers in doing leadership.

Having outlined the conceptual stance that I take on leadership, the rest of this chapter will focus on the analysis of how the leaders in the Prems go about constructing themselves as leaders, the role the other leaders play in this and the importance of the followers. As specified earlier, although leaders exist at all levels of the team, the main focus will be on the core members of the Leadership CofP, for the simple reason that as institutionally reified leaders, they have far more opportunity to speak in group situations than any other members of the Prems and thus were recorded more often. While other leaders are not excluded from the analysis, it is harder to exemplify the range of leadership behaviour that they exhibit compared with the "official" leaders. The following analysis is divided into three main sections, the first deals with back-stage constructions of leadership, that is, how leaders construct themselves when talking to other leaders. This also includes negotiating leadership decisions and strategising about how to lead the team. The second section examines how these leadership identities are then put into practice in the front-stage, and the final section examines how leadership practice can be renegotiated and the effect that a change in leadership personnel can have on leadership practices. 


\subsection{Constructing leadership back-stage}

Although the terms back-stage and front-stage, originating from Goffman (1959) and further developed by Richards $(2006,2010)$, are explained in the literature review of this thesis, this is the first point at which they are used as central concepts in the analysis. If we consider the whole scope of interactions that take place within the Prems as a stage, then certain interactions are performed to the whole team or subsets thereof, such as huddles, team meetings and explanations of training drills. These are considered front-stage in a team context. The interactions that take place outside of these performative spaces can be categorised as back-stage. Back-stage interactions are not public to the whole team; they are typically conversations including two or three individuals. However, they are often pertinent to the construction of identity front-stage as well, because it is here that much negotiation takes place between individuals, particularly in terms of relative status.

What this chapter is concerned with is how leadership identity is constructed backstage, between leaders and then reconstructed front-stage. Both can be considered as doing leadership, but the back-stage construction of leadership is often overlooked in analyses of leadership (Richards 2010). In organisations where distributed leadership strategies can be identified, however, an analysis of back-stage leadership provides insights into the stance that leaders intend to take when front-stage and the ways in which leadership status is negotiated between leaders. In the case of the Prems, the back-stage interactions that take place between members of the Leadership CofP can be considered one way in which they negotiate how to be a leader in the Prems and their relative positions in terms of core and periphery, as discussed in Chapter 4 . In fact, as the extracts presented here illustrate, it is through the participation in back-stage leadership discourse that a peripheral member of the Leadership CofP may move closer to the core.

Example 5.1 shows how back-stage leadership discourse can be used in making shared decisions. It also shows how two leaders with a power asymmetry, Tommo and Jon, negotiate a shared membership of the Leadership CofP. In the extract Tommo and Jon discuss how to go about disciplining some of the players who have recently been bringing the club into disrepute by drinking heavily, fighting, and getting arrested. 


\section{Leadership}

\section{Example 5.1: Tommo and Jon discussing discipline}

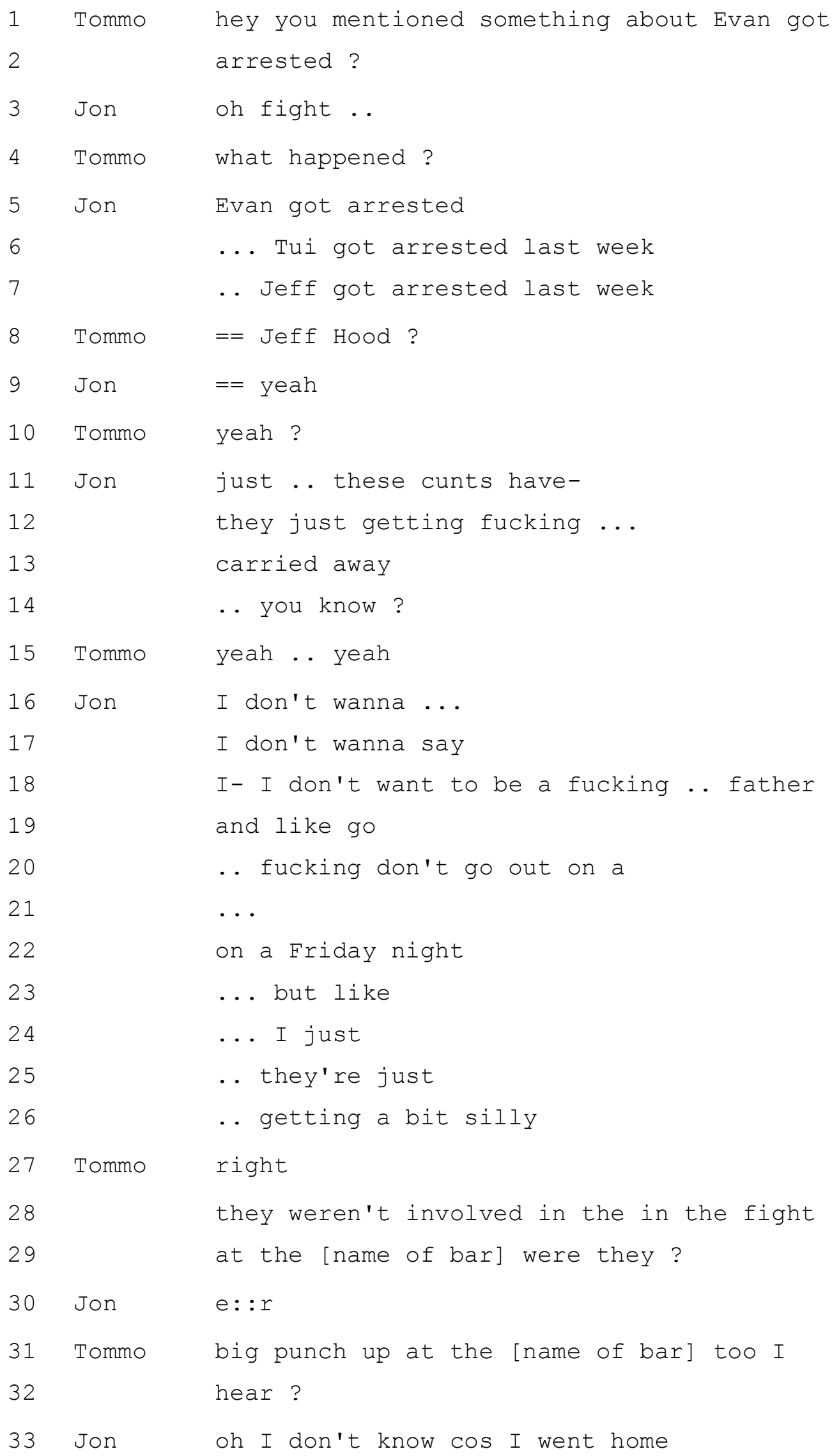




\section{N. A. Wilson}

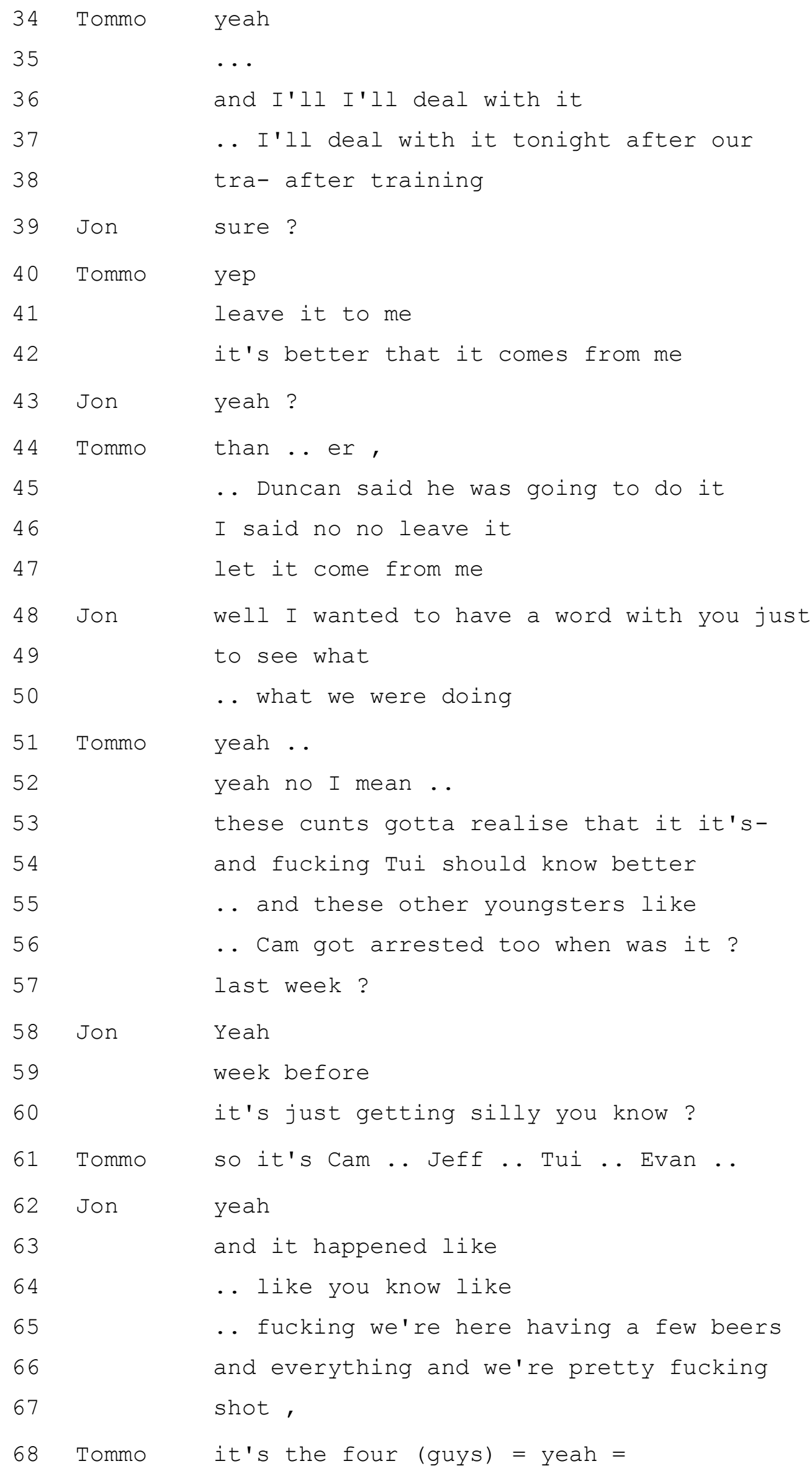




\section{Leadership}

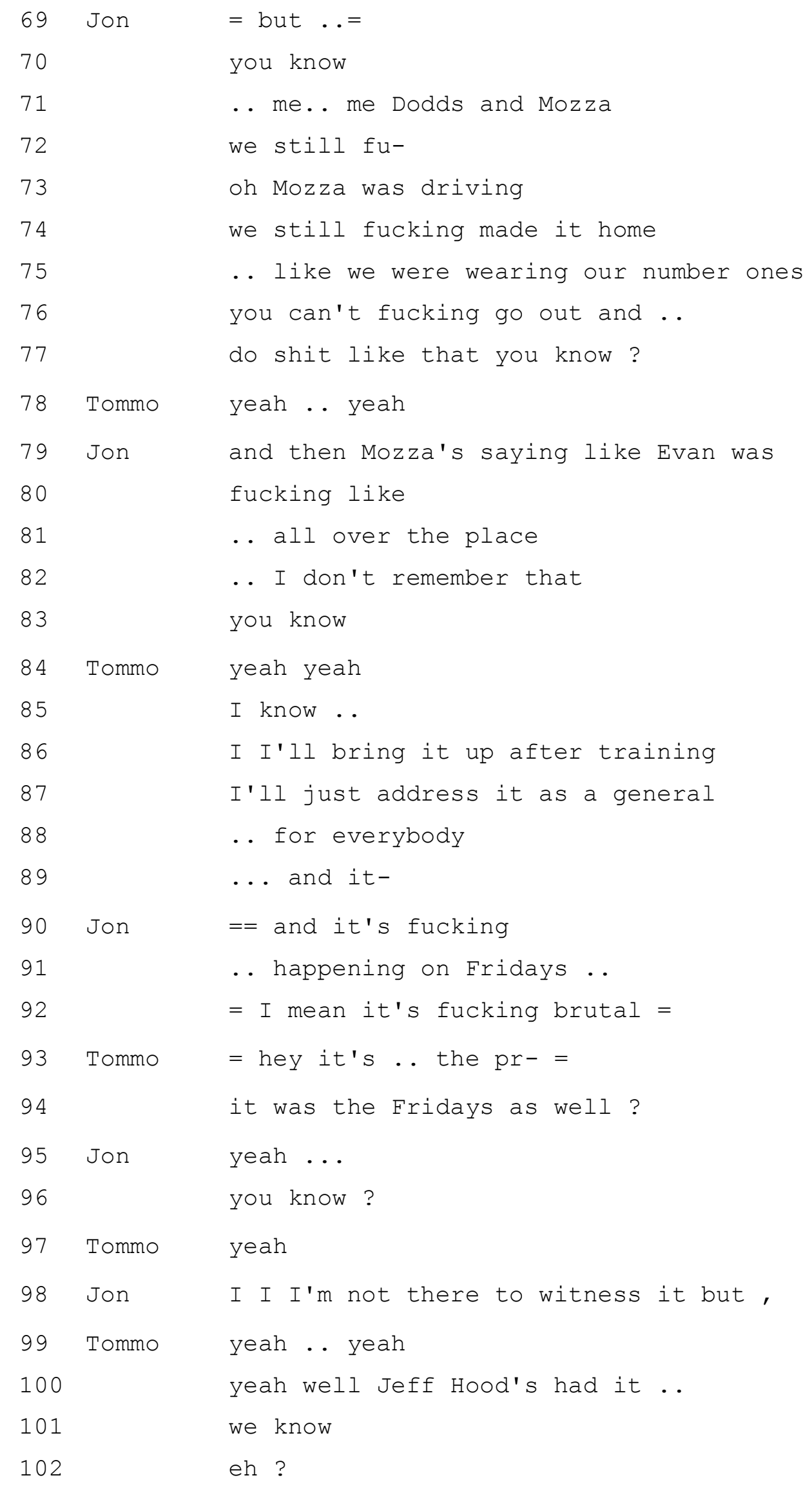


What is perhaps most interesting about this extract is that it features Tommo and Jon talking about the leadership identity they want to present to the players. For instance Jon states that he does not want to be a father (line 18) by telling players that they are not allowed to go out. However, Jon is also very careful to distance himself from their behaviour and makes it very clear that he disapproves, calling the players concerned cunts (line 11). Although not an uncommon word to hear from Jon, in all other occurrences that were recorded it is used to refer to the opposition, thus calling his team mates cunts is a strong statement from Jon. That Tommo echoes this later (line 53) is in part in agreement with Jon's evaluation of the players' behaviour but also may reflect a degree of accommodation (Giles et al. 1991; Schneider 2008) to Jon's expletive laden discourse style, with Tommo adjusting his discursive style to match his audience (Bell 1984).

It is noteworthy that it is Tommo who makes the opening move in this conversation, seeking information from Jon to confirm the rumour he has heard about a player being arrested. From a team discipline point of view this is a very serious matter and thus Tommo must find out exactly what has happened. Jon not only informs Tommo that three players were arrested but that the root of the problem is the culture of heavy drinking that exists among some of the players. By informing Tommo that this is now happening not only on Saturday nights after matches but on the Friday nights before them, Jon effectively aligns himself with the coaches over the players. He puts the needs of the team over the individual needs of the players as he knows that they will be punished as a result. In doing so Jon distances himself from the events, saying that he went home early (line 33) and making explicit the politic behaviour of players which is to behave when representing the club when wearing their number ones (line 75) (club tie and blazer). As in Example 5.2, where it is examined in greater detail, Jon uses oh to signal a negative evaluation of the players' actions (line 3) as well as to mark new or newly remembered information (lines 33 \& 73) (Schiffrin 1987; Trester 2009). He is careful however, in reporting the misbehaviour of others, to exonerate Mozza and Dodds, suggesting that they behaved as he believes a Prem should, as of course did he.

Once Tommo has heard the information he needs, he reassures Jon that he will deal with it (lines 34-38). Jon has expressed a reluctance to discipline the players, perhaps feeling that the leadership identity he has constructed with them would not legitimise 
such a move. This may be the reason that he provides so much information to Tommo. Tommo also informs Jon that he has known about the situation for a while, as has Duncan, the team manager, but that Tommo saw this as a matter that should be addressed by him (lines 45-47). This interaction indicates that Tommo is conscious of the different leadership identities that the various leaders within the Prems construct with the team and that a highly face threatening act such as an admonishment for disreputable behaviour outside of the club requires a great deal of mitigation in terms of hierarchical authority and solidarity, both of which factor in Tommo's leadership identity, which may be regarded as indexing the good mate archetype in situations such as this one. Reassured of this, Jon makes a move to fully align himself with Tommo in the leadership of the team by saying well, I wanted to have a word with you, just to see what what we were doing (lines 48-50).

Tommo later discusses the exact manner in which he will deal with the situation; he will address it as a general for everybody (lines 87-88). By discussing his strategy with Jon in this way, he acknowledges Jon's status as a co-leader and thus a core member of the Leadership CofP. This may reassure Jon that by not singling out players for punishment Tommo is minimising the chance of the players thinking that Jon informed on their actions. Moreover at the end of the conversation Tommo makes a final acknowledgement of shared leadership knowledge with the statement Jeff Hood's had it. We know. Eh? (lines 100-102). Jon's earlier high solidarity stance (lines 48-50) may be considered as having been accepted by Tommo from this statement. The use of we know can be interpreted as grouping the two leaders together through the use of the inclusive personal pronoun, and the final tag $e h$ ? is a marker of solidarity, informality and egalitarianism in New Zealand English (Bell 2000; Holmes et al. 2009; Meyerhoff 1994). Thus Tommo's acceptance of Jon's stance has the effect of constructing a shared identity for Jon and Tommo as leaders and, in this back-stage interaction, both the transactional goals of allocating leadership duties and the relational work of maintaining shared leadership relations are fulfilled.

While Example 5.1 deals with how back-stage leadership discourse can be used in making shared decisions and constructing a shared leadership identity, Example 5.2 
shows how the coaches use constructed dialogue ${ }^{17}$ and storytelling in the back-stage to create individual and shared identities as leaders.

In Example 5.2, the two coaches, Tommo and Parky, are the focus. As discussed in the previous chapter, Parky and Tommo create the initial norms of leadership discourse in the Prems. Furthermore their complex relationship appears to be continually renegotiated. Although Tommo is the head-coach and Parky the assistant coach, Tommo is younger and less experienced than Parky. Tommo volunteered in an early interview that he sees Parky as a coaching mentor, someone with whom he can check his ideas. As Parky's professional life is concerned with the professional development of rugby coaches, it is no surprise that he steps in to this role easily and can often be seen acting in this mentor capacity. However, he also fulfils his role as an assistant coach by doing more of the detailed work with the players, such as running warm-ups and analysing individual strengths and weaknesses. This takes place both in training, where he provides immediate feedback to the players and after matches, when he analyses a video of the match and compiles a statistical breakdown of the various performance indicators that are used to measure individual player performance. Thus, while Tommo does have the final say in all decisions, he tends to seek Parky's advice in team decisions and keeps him informed of any plans he has regarding training or gameplay. Example 5.2 illustrates Parky performing one of his detailed duties in leading the players through a fitness drill. This drill has been assigned to the players as a form of punishment for poor match performance. At the beginning of the extract Tommo has just arrived (late) while Parky has been running the training session for about twenty minutes.

\section{Example 5.2: The Justin problem}

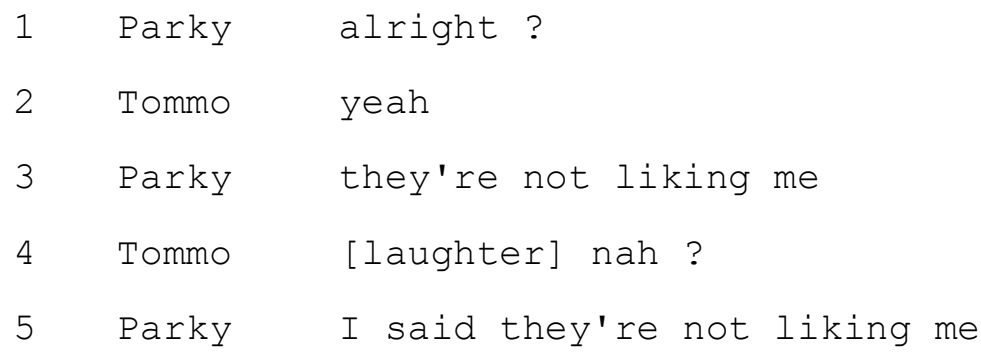

${ }^{17}$ Following Tannen (1986), I prefer the term constructed dialogue to reported speech, because this conveys better the sense that storytellers often use some poetic licence with the way in which they report conversations, either to present themselves in a better light, or for dramatic effect. However, both terms refer to the same phenomenon and, reflecting the widespread use of each, they are used interchangeably. 


\section{Leadership}

\begin{tabular}{|c|c|c|}
\hline 6 & Tommo & $==$ oh that's alright they'll get over it \\
\hline 7 & Parky & yeah I know \\
\hline 8 & Tommo & I just had a um - \\
\hline 9 & Parky & $==$ we did a (ball lining) warmup \\
\hline 10 & & and they were fucking atrocious \\
\hline 11 & Tommo & just attitude or just switched on- \\
\hline 12 & & not switched on ? \\
\hline 13 & Parky & just not switched on \\
\hline 14 & Tommo & $\{[$ whispered] (look at this) $\}$ \\
\hline 15 & & $\cdots$ \\
\hline 16 & & just had a rather heated discussion with \\
\hline 17 & & Justin Peters \\
\hline 18 & Parky & oh (was it) ? \\
\hline 19 & Tommo & yeah in the tunnel \\
\hline 20 & Parky & oh I just said to him- \\
\hline 21 & & look he came and he said \\
\hline 22 & & . oh what's your list like ? ${ }^{18}$ \\
\hline 23 & & I said I don't know yet Justin \\
\hline 24 & Tommo & oh he just came out and he goes \\
\hline 25 & & oh.. you've you've brought one of the \\
\hline 26 & & colts up.. to sit on your bench \\
\hline 27 & & I went $\ldots$ \\
\hline 28 & & and who would that be? \\
\hline 29 & & . he says oh that guy you played \\
\hline 30 & & .. that back \\
\hline 31 & & .. I says \\
\hline 32 & & oh no... if he's up with us he's starting \\
\hline 33 & & .. oh well how can you bring a colt up to \\
\hline 34 & & start when we can't have him to play? \\
\hline 35 & & .. I said well the club's policy is \\
\hline 36 & & .. that school leavers are encouraged \\
\hline 37 & & .. to play colts unless they are of \\
\hline
\end{tabular}

18 Justin's reported speech has been italicised to indicate the marked change in voice when this occurs and to make it easier to distinguish it from sections when the coaches report their own speech. 


\section{N. A. Wilson}

41 Tommo

Tommo premier standard

well that's no benefit to the club

Parky right

he said senior ones are g- we're gonna

fall over.. because we haven't got a

first five

.. I said Justin .. I can understand what

you're saying

.. I said .. but it's not my decision ..

that's the policy that the club has

well that doesn't benefit the club

.. and he got all-all

. antsy over it

... I said mate if you don't like the

policy

I said .. there's a committee meeting tomorrow.. go to address it with them

I just said I'm telling you /

Parky I said I said to him I said

I don't know whether stuart will be with

us yet or not

.. I said if he's gonna be o-

if he-

if he's has to be on our bench

.. then it would make sense that he plays

in your game beforehand because the

twenty-ones are playing here

but I said

if we're not going to use him

.. then he'll play in the colts here

oh .. so he's already asked you and now he's trying to twist it $=$ round on me $=$

Parky = yeah he's = trying to play us off

against each other mate

Tommo fucking dick...

he got no change though 


\section{Leadership}

97 Tommo

98 Parky 99

101 Tommo he's just got upset

... you know ..

we're- we're looking at seventy point

hidings because we haven't got a first

five

.. I said

mate we haven't got a goal kicker

.. do you see us..

you know...

bitching and moaning ?

you've got to make do with what you've got

.. oh we're going to lose players

I said

keep them (alive) get them doing things

Parky I mean

we already had one or two of them like

Ian last Thursday

when we were going to do a team run

I said Ian ... I-

you can go and train with the senior ones

he said..

that's shit training I rather stay here

yep well

$\{$ [to players] you're up to twelve

remember guys

.. twelve of each\}

Cliff was exactly the same

.. and he's..

probably the best club man we've got here

.. and he'll do it

and he says

oh ... if I go there

I've got to run training 
The opening segment of the conversation (lines 1-13), while fulfilling the function of a greeting and solidarity enhancing small-talk (Mirivel and Tracy 2005), is also used by Parky to convey to Tommo the leadership identity that he has constructed with the players in leading them through this drill. He tells Tommo that the players are not liking me (lines 3 \& 5), thus indicating that he has taken a tough stance, deliberately losing popularity with the players in order to make them do fitness work, an unpopular but accepted part of training. Although the decision to do fitness training had been jointly taken by the coaches on an earlier occasion (Tommo informed me in an interview that he and Parky always jointly plan the content of the training sessions in advance), Parky further justifies the need to play "bad cop" by explaining that the players had already been performing poorly in training (lines $9 \& 10$ ). Between them Parky and Tommo then establish that this is down to lack of concentration rather than attitude on the part of the players (lines 11-13), although as the events that follow this conversation show (these are analysed later in this chapter), Tommo's suggestion that it was down to poor attitude (line 11) may have been accurate. In relation to the archetypes described earlier in this chapter, Parky's leadership style with the players in this situation could be compared with the father archetypal leader, admonishing players, but with the goal of making them learn from their mistakes. This style will become more evident in Example 5.3 and Example 5.4 which show how events develop in this training session.

After the opening exchange Tommo then recounts a heated discussion (line 16) he has just had with Justin, the coach of the Senior Ones. In part this may be interpreted as a reason being given for his lateness. However, as the narrative progresses and is coconstructed by Parky, it becomes clear that this is more than just an explanation. It seems that Tommo is very keen to tell Parky about this, as shown by his initial attempt to tell him at line 8 before Parky has finished talking about the poor focus of the players. Just as Tommo has reassured Parky about the tough stance he has taken with the players (line 6), it may be that he seeks reassurance that the stance that he took with Justin was justified. Furthermore, as Tommo and Parky share the coaching leadership of the Prems, it is important that Tommo tells Parky anything that has happened that might impact on team selection. However, before Tommo details his full conversation with Justin, Parky makes him aware that he also had a similar discussion (lines 20-23). This fulfils two relational functions between Parky and Tommo. Firstly, it shows 
empathy; Parky understands the situation Tommo was in as he too had been in it. This has been noted in other research as a collaborative discourse strategy stereotypically associated with women's talk (Fletcher 1999; Lakoff 1973; Tannen 2001); its use here may indicate that Parky employs a range of relational strategies to do relational work, and that Parky acknowledges that one of the functions of Tommo's narrative is to provide catharsis in unloading his feeling of annoyance at Justin. Secondly, the swapping of stories constructs a group boundary between the coaches of the Prems and Justin, positioning Justin as an outsider who has made a discursive move that his position in the club hierarchy does not justify. By sharing their experiences of an outsider attempting to interfere with their leadership of the Prems, Parky and Tommo reinforce the supportive norms of the team and the Leadership CofP. This is highlighted with the reference to the confrontational aspects of Tommo and Justin's heated discussion (line 16). Through Tommo's reference to his bluntness, Justin is constructed as not communicating a request between coaches appropriately: he just came out and he goes (line 24). He is further constructed as being unreasonable, and perhaps a little petulant, when Tommo describes him as getting antsy over it (line 49). The construction of Justin's attempt to subvert the authority of the Prems coaches as a minor annoyance is shown in Tommo's summary of the effect of Justin's actions as resulting in nothing more than getting Justin upset (lines $73 \& 74$ ). However, while Tommo is quite pragmatic about the general outcome, his negative evaluation of Justin's behaviour is shown most strongly when he discovers that Justin has tried the same strategy with both Tommo and Parky, calling him a fucking dick (line 71). This is notable because although the communicative norms of the Prems allow (or even require) a high frequency of swearing, there are relatively few uses of swearing as insults other than as a form of jocular abuse, which tends to be indicated by a co-occurrence with laughter. Those instances of insults with swearing that do occur without laughter, like this one, are almost exclusively directed at a third party who is not present. For instance in prematch huddles the opposition are often referred to as cunts (e.g. Example 7.3), but players and coaches rarely use swearing to legitimately (i.e. not jocularly) insult speakers that are present.

In addition to the explicit evaluation of Justin's behaviour and communicative stance described above, the use of discourse markers in reporting the speech of Justin can be 
interpreted as indicating the stance that each takes towards Justin's demand that high standard Colts players should play for the Senior Ones rather than the Prems. Parky uses the standard reported speech marker said (lines 21) to mark both his own and Justin's speech exclusively, over any other form of quotative marker. Tommo on the other hand, while using said a comparable amount also makes use of a wide range of quotative markers, including says, went, goes, and the zero quotative (D'Arcy 2010; Holmes 1998b). While his use of what are typically regarded as non-standard markers of reported speech may index the difference in age between Tommo and Parky. It may also be a marker of social class. However, Tommo's use of the zero quotative is interesting. This has been shown to be a marker of Māori ethnicity (D’Arcy 2010; Holmes 1998b), yet although Tommo tends to use some linguistic features that index Māori identity (such as te reo Māori words like koro, meaning "respected elder", when addressing the oldest player in the team), he is Pākehā. One possible explanation for this is that "ME [Māori English] is used by some Pākehā who mix socially with Māori people" and "Pākehā whose social network ties are predominantly Māori, or who have positive attitudes toward Māori people, often use ME as a signal of solidarity" (Holmes 2005c:93). Thus it may be that although Parky is Pākehā, as is Tommo, over half of the players are Māori and some Māori norms and linguistic features are embedded in the team's linguistic repertoire. Ethnographic work revealed that Tommo's social network ties outside of the rugby club are indeed predominantly Māori. Tommo may use Māori English features, in this case the zero quotative marker, in order to align his identity with the team and create solidarity with other team members, whether they are Māori or Pākehā. Furthermore, the range of devices that Tommo uses for reporting speech may be a reflection of the wide discursive repertoire he has at his disposal and the range of resources available to him, creating a variety of leadership styles used in backstage and front-stage situations.

In addition to the range of quotative markers that Tommo employs, he also prefaces many constructed dialogue items with oh (lines 25, 29, 32, 33, 86 \& 106). Parky also uses this discourse marker, but only once to preface reported speech. It has been shown that this can indicate a negatively evaluated stance (Clift 2006; Heritage 1998; Trester 2009). In other words, by prefacing Justin's speech with oh, both Tommo and Parky convey the level of disagreement that their respective encounters contained. However 
on one occasion (line 32) Tommo also prefaces his own reported speech with oh. As it is unlikely that he negatively evaluates his own stance, oh appears to further underline the opposition of the speakers in the constructed dialogue to each other's stance. On the other hand Holmes (1998b) points out that the lack of lexical framing of reported speech that is associated with a Māori narrative style can mean that discourse markers like well (line 48) and oh (lines 33 \& 86) are used to assist in indicating speaker change. Thus it may be that the use of $o h$, while in part evaluative, is also part of Tommo's discourse style that indexes a Māori identity and team solidarity.

However, these functions of $o h$ are not the same as when it appears outside of constructed dialogue, which it does many times in this conversation. When turn-initial, it seems to indicate that what the last speaker said is new information and that following speech will provide what the speaker knew from their experience (Aijmer 2002; Heritage 1998; Schiffrin 1987). This is also a function within the boundaries of reported speech, in addition to its evaluative function (Trester 2009) and its identity indexing properties (Holmes 1998b).

Each coach thus constructs Justin in such a way as to make it clear that they opposed his complaints and criticism, and reinforces the boundary between them and the coaches of other teams in the club. By creating an out-group opposition (Baxter and Wallace 2009; Duszak 2002; Richards 2006; Wieczorek 2009), Tommo and Parky strengthen the solidarity between them, but they also show that they are in agreement on how to handle Justin's attempt to play them off against each other (line 71). Perhaps the most important outcome of this conversation is that through their exchange of narratives, the two coaches work out Justin's divisive strategy (lines 68-71). Once this transactional outcome has been achieved however, they continue to swap pieces of information in a way that might be regarded as gossip, creating an identity of the Senior Ones as disorganised and lacking in leadership (line 72-96), which further enhances their own relational bond. Tommo and Parky thus not only contrast their co-operative leadership strategy with Justin's divisive attempts to subvert their authority, but also construct Justin as an ineffective leader on the basis of reported complaints from his team, the Senior Ones. 
A succession of anecdotes and an us/them distinction (Baxter and Wallace 2009; Duszak 2002; Richards 2006; Wieczorek 2009) creates an identity for Tommo and Parky as equals in the leadership of the team, overcoming the mismatch between Parky's experience and official position in the hierarchy and uniting them against outside attempts to interfere with their leadership. Additionally, Tommo's use of nonstandard reported speech markers could signal an alignment to the team discursive norms and therefore lesser social distance while Parky's adherence to more standard forms may be interpreted as showing that he does not align himself in this way. Thus by displaying some discourse features that position them closer or further from the players, the two coaches set up different but complementary leadership identities in relation to their closeness to the team. Furthermore, these leadership identities seem to be congruent with the norms of solidarity or competitiveness that exist in each coaches positional CofP (as discussed in Chapter 4).

The two examples given in this section demonstrate that core members of the Leadership CofP co-construct each other's leadership identity through their back-stage interactions, using the discussion of how they intend to do leadership when front-stage (Example 5.1) and the stance they have taken when performing their leadership duties as a representative of the team. In addition, leaders can do back-stage and front-stage leadership in an overlapping manner. In the examples discussed in the next section, Tommo and Parky maintain a back-stage conversation while performing front-stage interactions with players and setting up their next front-stage leadership stance.

\subsection{Moving from back-stage to front-stage leadership}

This section features two examples that are taken from the same training session as Example 5.2. The first follows directly on from where Example 5.2 finished and is a continuation of the same communicative event between Tommo and Parky, albeit with a change of topic and discourse style. The second example in the section takes place twenty minutes later, at the end of the fitness drill. The intention is to show how the shared leadership strategy that is discussed back-stage in Example 5.3 is executed in Example 5.4. Furthermore, Example 5.3 shows the coaches moving seamlessly between front-stage and back-stage, giving instructions and encouragement to players while carrying on their conversation with one another. 


\section{Leadership}

\section{Example 5.3: Catching cheaters}

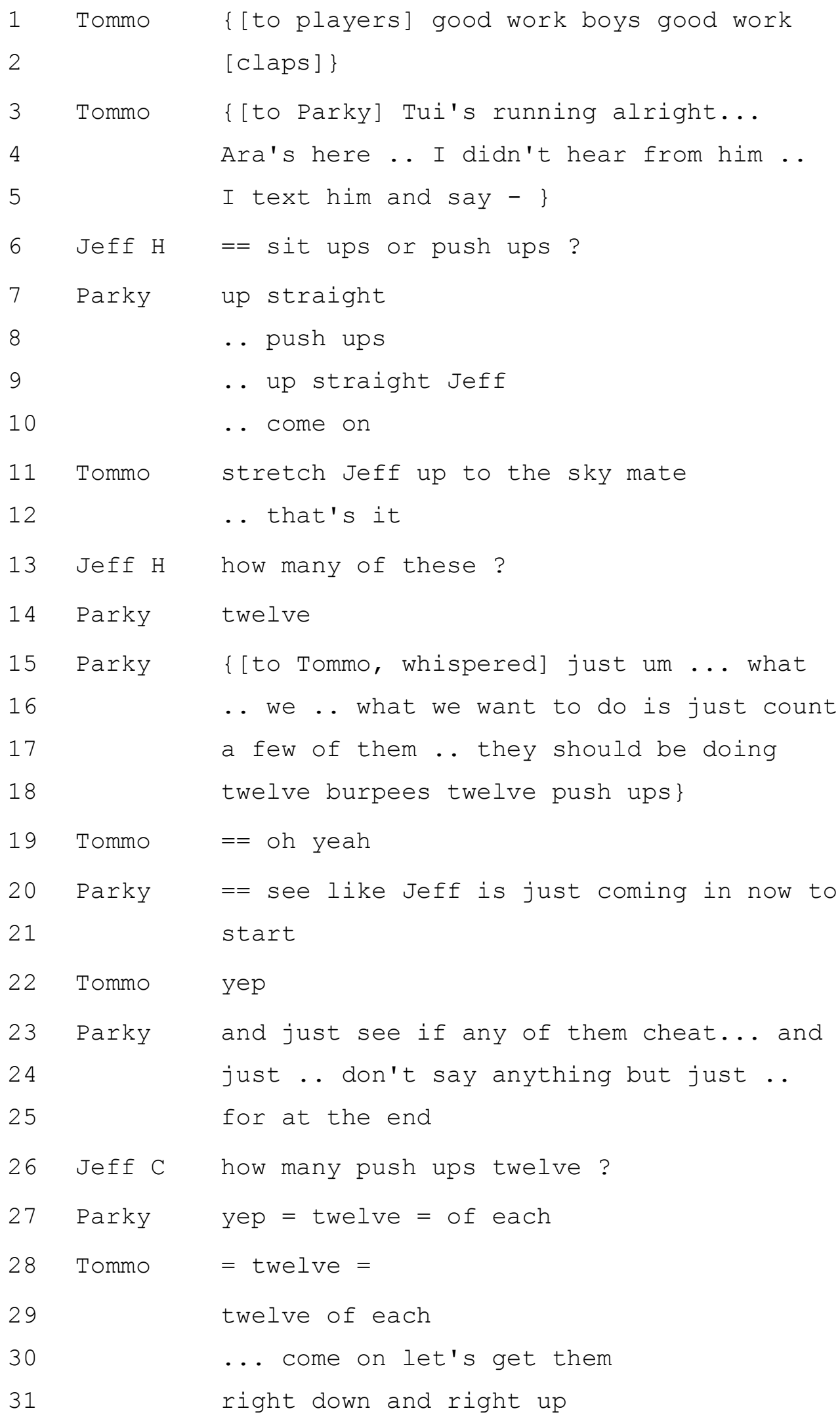




\section{N. A. Wilson}

In Example 5.2, when Parky greeted Tommo, he signalled that he had adopted a tough stance with the players. Example 5.3 shows that he also wants to catch the players out as he believes they will try to cheat (lines 15-25). Thus the fitness drill has multiple training purposes. Firstly, it is punishment for a poor performance in the previous match; secondly, it will improve the player's stamina; and thirdly, if the players cheat and the coaches catch them it will provide a lesson for the players on how their attitude does not match the coaches' expectations. In telling Tommo what he is planning (lines 15-25), Parky increases their solidarity by positioning Tommo as a confidant, similar to the way Tommo did with Jon at the end of Example 5.1.

Despite the equality and solidarity that was constructed moments before in Parky and Tommo's exchange of stories relating to Justin, it seems that Parky is in charge here as it is he who tells Tommo what to do. However, it appears to be a mark of their solidarity and equality that these implicit directives (lines 15-18) use both we and the qualifier just as mitigation in the act of one coach telling another what to do (Vine 2004). The use of just is repeated with the more explicit directives (lines 23 \&24) which are further qualified by Parky's somewhat cryptic explanation of for at the end. That both Parky and Tommo carry out this back-stage discourse of organising their coaching while also performing front-stage in their interactions with the two Jeffs shows that they can not only construct different front-stage leadership styles, but also perform relational work with each other, simultaneously. It is noticeable that Parky adopts a more authoritative style when addressing Jeff $\mathrm{H}$ by using bare imperatives (lines 7-10 \& 14), than Tommo, who uses a more relational style with the use of mate to the same player (line 11) and let's, to Jeff C (line 30). This is an example of Tommo's greater solidarity amongst the players and the two styles complement each other to a degree, with Tommo playing "good cop" to Parky's "bad cop".

The next example, which shows what happens after the players have finished the drill, further exemplifies the way in which Parky and Tommo juxtapose tough and supportive leadership identities, following through on the plan to catch out the players that cheated in the drill.

\section{Example 5.4: Cutting corners}

Context: The players are standing on a line marked on the pitch facing the coaches, having completed the fitness training exercise. 


\section{Leadership}

\begin{tabular}{|c|c|c|}
\hline 1 & Parky & those guys that did it in twenty \\
\hline 2 & & well done \\
\hline 3 & & .. what I'd like um- \\
\hline 4 & & what I'd like you to do \\
\hline 5 & & is anyone who took a short cut in that .. \\
\hline 6 & & anyone who didn't do \\
\hline 7 & & ... their burpe- \\
\hline 8 & & the correct number of burpees \\
\hline 9 & & .. or anyone who didn't do their correct \\
\hline 10 & & number of push ups \\
\hline 11 & & .. or anyone who didn't run \\
\hline 12 & & .. to the line \\
\hline 13 & & . so they cut the er length of the field \\
\hline 14 & & short by a metre or ( ) or whatever \\
\hline 15 & & .. I want you to stand on that side of \\
\hline 16 & & the line \\
\hline 17 & & [after about 30 seconds of deliberation \\
\hline 18 & & almost every player steps across the \\
\hline 19 & & line ] \\
\hline 20 & Tommo & anyone that if you you took any shortcuts \\
\hline 21 & & at all whether it's half a metre \\
\hline 22 & & or missed a burpee \\
\hline 23 & & or missed anything \\
\hline 24 & & that side if you didn't \\
\hline 25 & & this side if you did \\
\hline 26 & & [all players that hadn't previously \\
\hline 27 & & moved, do so now] \\
\hline 28 & Parky & okay what's that telling us guys ? \\
\hline 29 & Jeff C & (we're) cutting corners \\
\hline 30 & Parky & what does it tell us ? Will? \\
\hline 31 & Will & cutting corners \\
\hline 32 & Parky & where does that get us ? \\
\hline 33 & All & $\{[$ muted $]$ nowhere $\}$ \\
\hline 34 & Parky & okay .. \\
\hline 35 & & in the corner \\
\hline 36 & & [players assemble in corner of pitch and \\
\hline 37 & & drink some water. Tommo goes to them] \\
\hline
\end{tabular}




\section{N. A. Wilson}

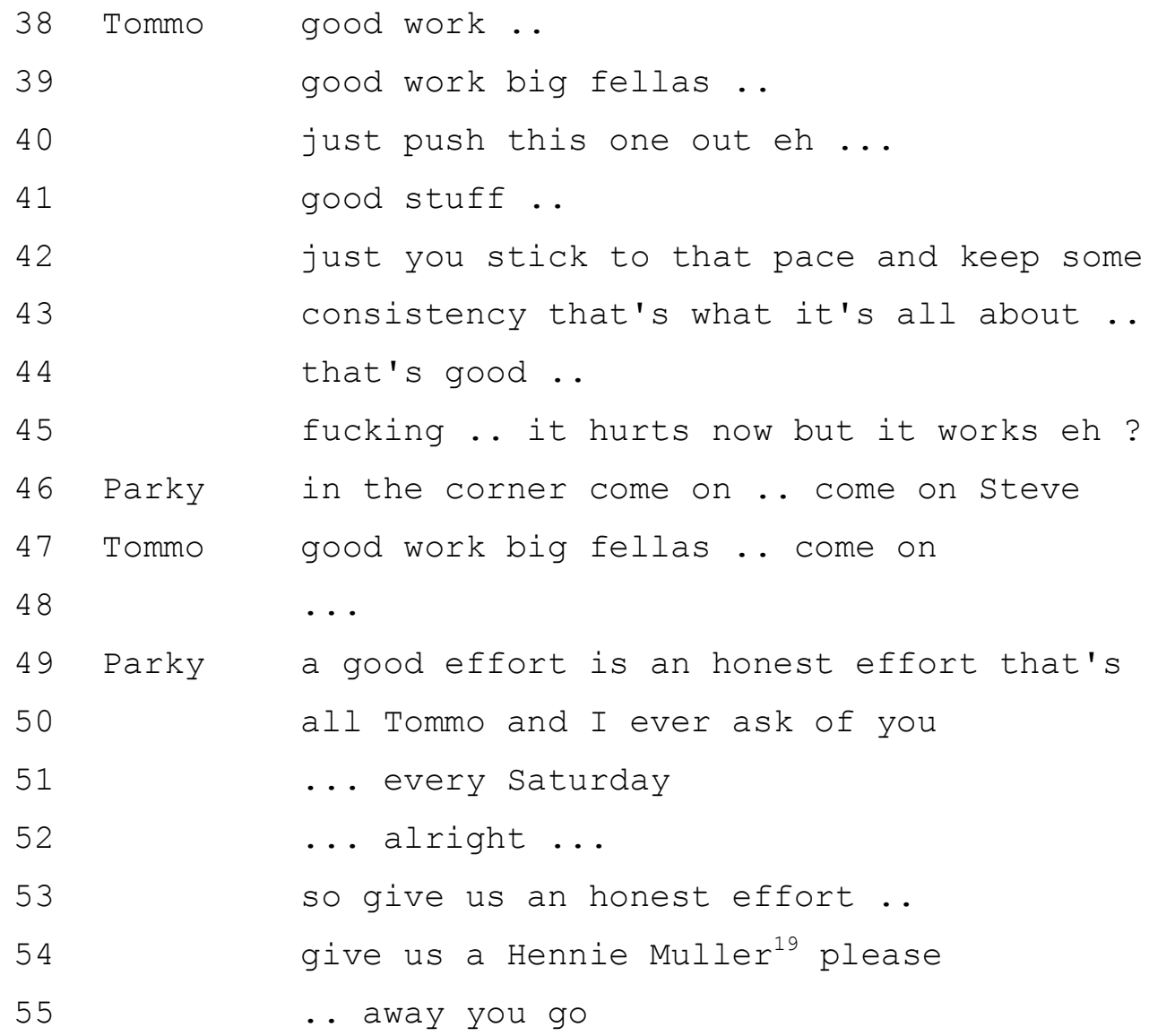

As in the previous example, Parky constructs a leadership style that is authoritative and pays little attention to face, exemplifying the stereotypical male leader and the father archetype. He uses I and you exclusively and although he does make use of the familiariser guys (as discussed in relation to player usage in Example 4.3), it is in the final position in a question thus prompting a response rather than performing any solidarity work (line 28) (Ervin-Tripp 1976; Wilson 2010). Not only does he use unmitigated directives as before, but he singles out players (lines $30 \& 46$ ), a highly face threatening act. One of the occasions on which he singles a player out is when he is asking the players to tell him what they have learnt. By singling out Will in this, despite the fact that Jeff had already answered, Parky suggests that Will may be one of the players who he considers should pay particular attention to this lesson. The strategy of asking players to repeat back to him their understanding of events or interpretations of

\footnotetext{
${ }^{19} \mathrm{~A}$ Hennie Muller is a punishment drill named after a famously hard-line South African coach which involves running along one length of the pitch, across the diagonal, up the other length and back across the other diagonal to the starting corner.
} 
the implications of training drills is a strategy that Parky uses frequently, often when training with the Backs. Using it with the whole team shows the front-stage leadership styles that Parky develops with one CofP can be used when working with the whole team and thus contributes to the discursive repertoire of the Leadership CofP. In fact it is a strategy that Jon also employs, but for a motivational function, as will be discussed in the next chapter.

Parky's questioning of the players precedes a change of focus in this communicative event, which is marked by his use of the discourse marker okay (Schiffrin 1987). He then directs the players to assemble in the corner (line 35), where the water bottles are located. Prior to this, and since finishing the twenty minute fitness drill, the players have not had the opportunity to drink any water and are all understandably thirsty. That Parky exercises control over when the players can drink in this situation is perhaps one of the greatest demonstrations of his undisputed power over the players in training situations such as these. It is also a further way in which he constructs his leadership in this situation as "tough".

Tommo on the other hand, supports Parky by clarifying his instructions and when all of the cheaters have been caught, addresses his CofP, the Forwards, referring to them as big fellas (lines 39 \& 47). He compliments them for their efforts which may be interpreted as undermining Parky slightly, but what Tommo could also be doing is repairing some of the damage that has been done in punishing the team as a whole. Although every player stepped across the line, this was a demonstration of solidarity rather than an indication of guilt (I was also watching who cheated). It may be that Tommo is covertly complimenting the Forwards on this display of solidarity, although it is impossible to know for sure. However, Tommo also supports the exercise in his comment it hurts now but it works eh? (line 45) showing that even though he may respect the solidarity of the players, they must still face their punishment - a Hennie Muller. In the final part of the extract (line 49-55) Parky constructs an us/them boundary between the coaches and players, which serves to further emphasise the coaches' position of power.

Example 5.4 clearly shows that the back-stage strategy that Parky suggested to Tommo in Example 5.3 of watching for who has cheated at the fitness drill, results in a front- 
stage leadership identity created for Parky of a hard-line, authoritative coach, while Tommo, although supportive of Parky's stance, repairs the damage that this approach may have caused to team morale. This works because Parky and Tommo communicate their leadership roles and decisions to each other back-stage. This allows them to present a united front when performing front-stage which also prevents outsiders (like Justin) from undermining their control over the team.

\subsection{Chapter summary}

This chapter has analysed leadership in the Prems using the Leadership CofP and the idea that leadership is a social practice rather than a combination of personality traits. In order to show the multilayered nature of leadership as it operates in a multilayered constellation of CofPs, I have invoked the concepts of front-stage and back-stage, showing that although the two are linked, the discourse and identity that is constructed in each can take different forms. Furthermore, it has been shown that the most important strategy for doing leadership in the Prems is to use a form of distributed leadership. This is true for the coaches, the coaches and captains, and for the captains among the players. Although it can be argued that the distributed leadership practiced by the coaches is more equally shared than that practiced by the players, it is also worth noting the role of the players in accepting and thus legitimating leadership as social practice. In performing a distributed form of leadership each leader uses different but complementary discourse strategies, whether it is the use of team-oriented vernacular language which conforms to high solidarity norms (Example 5.2), or strategies which maintain distance from the players and perform overt displays of power (Example 5.4).

The discussion of the leadership literature at the beginning of this chapter outlined a number of archetypal male leadership identities in the form of the hero, father and best mate. This chapter has shown that in some situations the leadership identities that are constructed bear some similarity to these. However, what is perhaps more interesting is the way that leaders construct identity when enacting distributed leadership, for instance one playing the father-figure and the other the apprentice (Example 5.3). Furthermore, in line with other research outlined earlier, this chapter has described how leaders construct a range of leadership identities through their interaction with their team mates and that while these may be compared with leadership archetypes, the 
construction of leadership identity is dynamic and highly dependent not only on the context of the interaction, but also on the reaction of the followers.

This chapter has discussed the construction of leadership identities. Chapter 6 focuses on the construction of team identity and explores the relationship between leadership identity and team identity. In addition it provides a more in-depth discussion of the use of stance and indexicality and examines how global ideologies are renegotiated as local practice in the rugby team. 
N. A. Wilson 


\section{Chapter 6 Indexicality, Ideology and Identity}

Identity, from a social constructionist view, is crucially dependent upon the context of the discourse through which it is created. In the Prems, the players construct identities as rugby players as a part of their locally negotiated discourse practices. However, much of the discourse that achieves this indexes ideologies and stereotypes that are culturally embedded in rugby as socio-cultural knowledge (Ochs 1993). This knowledge is available to all rugby players as a discursive resource and signalling it helps construct an identity as a rugby player. Thus ideologies that exist on a global, macro level can be renegotiated locally and incorporated into an identity for the team and define a speaker's position within that team, or in CofP terms, their degree of integration to the core.

This chapter examines the rugby ideologies that are indexed in the discourse of the Prems and contrasts the way in which these are negotiated as a part of team identity with existing research in which they are also described. In addition to the construction of team identity that occurs through the indexicality of rugby ideologies, macro level identities such as masculinity are indexed through the discourse styles and norms of interaction that are negotiated as social practice within the Prems. Thus, not only does identity exist on multiple levels, but the ways in which it is constructed through discourse are also multi-layered. The section that follows further explains the principles of direct and indirect indexicality that were introduced in Chapter 2, as these are the concepts around which this chapter is organised, drawing upon concepts of nth order indexicality (Silverstein 2003) and layered simultaneity (Blommaert 2005). Following this, examples are analysed that highlight the contribution of rugby ideology and macro identities to the Prems' identity as a rugby team and the discourse practices that index these identities and ideologies through stance.

\subsection{Stance, indexicality and ideologies}

In social-constructionist terms, identity can be thought of as the accumulation of stances taken over time within a group (Jaffe 2009:11), stance being the dynamic instance of 


\section{N. A. Wilson}

identity formation (as discussed in Chapter 2). Stance is more than just linguistic style however, as well as indexing macro social identities such as gender and ethnicity through the use of indexical discourse features, it positions both the interlocutor and addressee in relative roles, for instance as leader and follower. In the negotiation of identity, stance is the opening gambit.

The theoretical framework of stance, which much of this thesis draws upon, views identity as being "defined within social formations" (Jaffe 2009:8) and proposes that in taking up a position, for example as a captain of a rugby team, "individuals automatically invoke a constellation of associated social identities" (Jaffe 2009:8). Such identities, considered from the point of view of the Prems might include being male, being a New Zealander, being Māori, being a rugby player, being a Wellingtonian, being a member of the rugby club, or being aged twenty-one. All, some or none of these might be indexed by a particular discourse feature used in adopting a stance. For instance, the frequent swearing that has been remarked upon previously as part of the shared repertoire of the Prems indexes the macro-level identities of masculinity, being a rugby player, and being young. However, rather than a discourse feature directly indexing a macro-level identity, the principle of indexicality (Bucholtz and Hall 2005) suggests that it is a stance with local meaning, such as solidarity towards the other players, that is indexed directly by swearing. This in turn indirectly indexes other identities. It should be reiterated at this point that indexicality is intersubjective: a local meaning derives from local understanding. In other words an utterance is framed by an addressee with reference to their socio-cultural understanding of the context and the speaker's intentions. As highlighted in Chapters 2 and 3, an ethnographer seeks to understand interactions from the point of view of the native. I am thus able to interpret the interactions within the Prems using a combination of my own native intuition as a former rugby player and the socio-cultural knowledge gained from my extensive interactions and participation with the Prems. This is triangulated with checks on interpretation provided in player interviews, and ultimately, because I was able to view every interaction in context. In other words, I was aware of what led to the interaction, the relationship between the speakers and to the ultimate consequences in the team of the interaction, or series of interactions. It is for all of these reasons that I have a 
justifiable warrant for my interpretation of the indexical meanings created in the interactions analysed in this chapter and elsewhere.

The indexicality can be categorised into two types, direct and indirect. Direct indexicality is when a feature of discourse invokes an unmediated link with a discourse stance (Bucholtz and Hall 2005; Ochs 1993). For instance, as shown in Chapter 4, the repetition of compliments in the Forwards indexes a supportive stance. Indirect indexicality is when this stance in turn indexes an identity, or accretion of stances (Bucholtz and Hall 2005), such as membership of the Forwards. This can be taken beyond the local levels of identity, however, if a discourse feature indirectly indexes an ideology embedded in the socio-cultural context. In rugby terms this might be the sporting ideology that winners should be gracious in defeat but magnanimous in victory (Gruneau 2006), or that rugby is a "man's game” (Ferguson 2004; Nauright 1996; Nauright and Chandler 1996; Phillips 1996; Pringle 2001).

Ideologies indexed by a stance may also index other, macro-level identities such as gender. This might be considered a multi-level indexicality or to rephrase this in Silverstein's (2003) terminology, a third order indexicality (where the first order indexicality is the local stance indexed by a linguistic phenomenon, and second order is the socio-cultural ideology). Silverstein (2003) posits that all indexicalities may be viewed in this way, giving a potentially limitless chain of identities and ideologies that may be indexed through discourse. This is observable to a degree in rugby, as some of the ideologies that form the socio-cultural knowledge of the sport are aspects of hegemonic masculinity, due to the enduring male control of rugby in particular and sport in general (Anderson 2009; Dunning 1986; Messner 1995). They may also, however, index national identity. For instance, many of the ideologies that define the archetypal rugby player also appear in such hegemonic masculinity archetypes as the New Zealand "Southern Man", who is tough, taciturn and rural (Law 1997; MacLean 1999; Phillips 1996). In New Zealand these identities are closely linked at the ideological level, whereas in other countries other masculine identities are associated with rugby. For instance in England, rugby is associated with the public school system and the "old boys' network", which indexes a hegemonic masculinity that occupies positions of institutional power (Schacht 1996; Sheard and Dunning 1973). Of course, 
there are also other macro-identities that are indexed by the various ideologies of rugby and these are examined as they appear in the analysis contained in this chapter.

While there are many ideologies that form part of the global socio-cultural context of rugby, the Prems do not draw upon all of them and it is likely that no team does. Only those ideologies that the coaches and players of the Prems index through their stance are described in this chapter and from these it is shown how they negotiate their own local identity. The rugby ideologies that are present in the team's discourse however, can be split into two main categories: those that are indexed through the teams' stance towards their locality, and those that are indexed through the teams' stance towards their identity as rugby players (which also indexes a masculinity). These ideologies are all indexed by way of stances that position players within the context of the team; the negotiated identities that result from these multiple stances are what may be regarded as team identity. Thus it is by indirectly indexing multiple layers of identity that the team creates an identity that can itself be indexed in creating additional stances that serve to further the goals of the team.

\subsection{First order (direct) indexicalities}

In Chapters 4 and 5, locally constructed stances were discussed in the form of the membership of the various CofPs and leadership stances. These appear to be present in all front-stage aspects of the Prem's discourse, in that there is always at least one person taking a leadership stance. His discourse and that of the other participants in any communicative event are bound by the communicative norms and expectations of their CofP and the event in question. Thus, it may be understood that much of what has already been presented in this thesis can be regarded as first order indexicality, and isconcerned with how discourse features and styles construct stances that have locally negotiated meaning within the team. This chapter looks beyond these local stances to explore how they relate to the socio-cultural context of rugby, both in its New Zealand context and as a wider social construct. Therefore, the first examples to be analysed address the next level of identity, the identity of the team in relation to its locality.

\subsection{Second order indexicalities}

Of the rugby ideologies evident in the Prems' discourse, some may be labelled as territorial; indexing three ideologies that are central to rugby and other team sports 
(Falcous 2007; MacLean 1999; Mizruchi 1985). These are the ownership of the home ground, the home advantage, and a metaphor of sport as war which positions the opposition as invaders. These are most often indexed in pre-match events, as shown below.

Territory is a fundamental part of rugby union, as MacLean points out (2004:9):

Unlike many other football codes, there is a strict demarcation of each team's territory and a rigidly enforced offside rule. The objective of the back-line, usually spread across the field, is two-fold: prevent the other team's penetration of territory, and occupy as much of the other team's territory as possible.

In addition to the territorial nature of the game of rugby described here, the way in which amateur rugby teams, like many other sports teams, are inherently linked to their locality can also be considered an aspect of territoriality. The rugby club is a place imbued with meaning derived from the local community in which it is situated (Bale 2001; Becker 2009). Club rugby is the "grassroots" and there is indeed a connection with the land, in that the team identity is fundamentally linked to the locality of the club and in many areas, particularly rural localities, the rugby club is a focal point for the community (MacLean 2004; Ryan 2005b; Tonts and Atherley 2010). Thus the identity of an amateur rugby team is bound up with its locality, and this section will demonstrate how the players draw on this local identity and sense of place in the build up to home matches.

Playing at home is thought to be an advantage as the players are familiar with the facilities and can expect a greater turnout of their fans to support them. Bale writes of football $^{20}(2001: 71)$ :

20 i.e. soccer. Although "football" is used to refer to a wide variety of sports and is the hyponym for the various different varieties that exist such as Rugby Football, Australian Rules Football, Association Football, Gaelic Football and American Football, it is used here in the sense it has in current New Zealand usage. However, and somewhat confusingly, in the past "football" has been used to refer to rugby in New Zealand. 
Players generally prefer home games because their team is likely to secure more wins, both scoring more and conceding fewer goals while playing at home. This widely observable outcome is known as the 'home field advantage' and has been shown to exist in a number of sports in a number of countries.

Indeed research in American sports such as baseball and ice hockey has shown that the motivational influence of home fans on players can have a major influence on the match result (Mizruchi 1985; Moore and Brylinsky 1995). Other research (Malmberg 1980) has shown the home ground to be the source of an advantage due to the territorial instinct to protect one's home (Morris 1981; Pollard 2008). While one might think of this as compelling evidence for the reality of the home team advantage, I analysed the match results in the league in which the Prems played finding that being the home team only correlated with winning half of the time. This is perhaps explained by the disparity between the top teams in the league and the bottom, a factor which Mizruchi (1985) mentions as being significant: the greater the difference in skill level, the less impact home advantage has. Van Houtum and Van Dam (2002) point out that territorial identity, constructed through an 'us versus them' discourse, is present for supporters at local, regional and international levels of football in Europe. Through the ethnographic fieldwork carried out with the Prems, it is apparent that an identity is created by indexing the territory of the home ground, as shown in the following examples, which are taken from different home matches. The Rock is the nickname for the team's home ground.

\section{Example 6.1: Invaders}

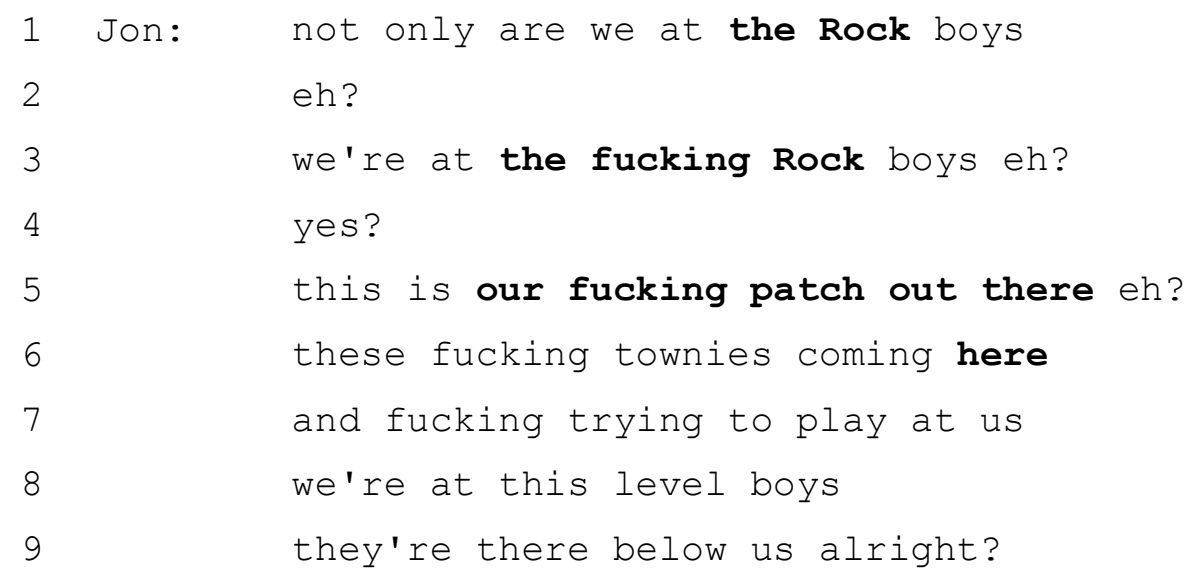


Example 6.2: Home-field advantage

$\begin{array}{ll}1 \text { Jon: } & \text { fucking [opposition team name] boys } \\ 2 & \text { they- fuck they hate coming out here } \\ 3 & \text { and we're in the fucking Rock } \\ 4 & \text { this is our fucking patch eh? } \\ 5 & \text { we fucking dominate the shit here eh? }\end{array}$

The reference to the home ground and the fact that other teams, especially from town (i.e. the nearby city) don't like coming out to play there is such a strong theme that it also appears in the team meeting, although without quite the same intensity as in the pre-match huddle (see Chapter 7).

In Example 6.1 and Example 6.2 there are several references to our patch. This is a clear statement of territoriality, claiming ownership of the home ground. In addition to this there are references to the opposition coming (out) here (Example 6.1, line 6 \& Example 6.2 , line 2), demonstrating the idea of the opposition invading and the home team protecting their home turf. Leaders in the Prems use territoriality in this way to motivate the players, both by suggesting that being at home is an advantage and by stressing the ownership of the territory. By linking the team with the home ground they index a stance of territoriality that both derives from and reinforces the territorial ideologies of rugby.

As these examples demonstrate, Jon, as captain, directly indexes the geographical location of the club, the link that the team has with the local community and constructs an identity for the team that involves ownership of the home ground. By linking the locality and the team, the coaches and captains provide a strong foundation for the team's identity, indexing an identity based on a place that already exists and to which all players can lay claim as locals. As Tommo said in interview: that's what you'll find with these guys .. that they'll actually .. play .. and give it everything for their club cos it's their community pride. The link with the local area is a powerful motivating force as well as a component of the players' and team's identities. Although created externally from the team (in forms such as the local newspaper and conversations between locals and players outside of the club), there is a link between the local community and the team which is indexed and thus incorporated into the team's identity. 
However, territoriality is not the only aspect of rugby ideology that is drawn upon in constructing team identity. There are numerous other ideologies that are indexed by the Prems. The difference is that as well as indexing rugby ideology and thereby contributing to the construction of an identity situated within the rugby world, these ideologies can also be regarded as indexing masculinities and as a such may be regarded as third order indexicalities.

\subsection{Third order indexicalities}

Many of the ideologies of rugby can also be viewed as ways in which hegemonic masculinity is constructed through rugby (Anderson and McGuire 2010; Light and Kirk 2000; Nauright 1996; Terret 1999). Example 6.3 provides evidence of a number of masculinity-indexing rugby ideologies that are used in the pre-match discourse of the Prems, namely control, violence and toughness (Anderson 2009). Violence is explicitly indexed through exhortations by Jon and Tommo to hurt the opposition, and toughness through the reference to the rugby ideology of tolerance of pain and injury. Control is linked to both of these as it is through control of aggression and control of pain that the masculine identity indexed is made specific to rugby (Bryson 1987; Fenton and Pitter 2010). All of these themes are apparent in Example 6.3, taken from a half time huddle at the point where the Forwards and Backs have split into two mini-huddles to discuss matters specific to their positions. As always, Tommo is with the Forwards.

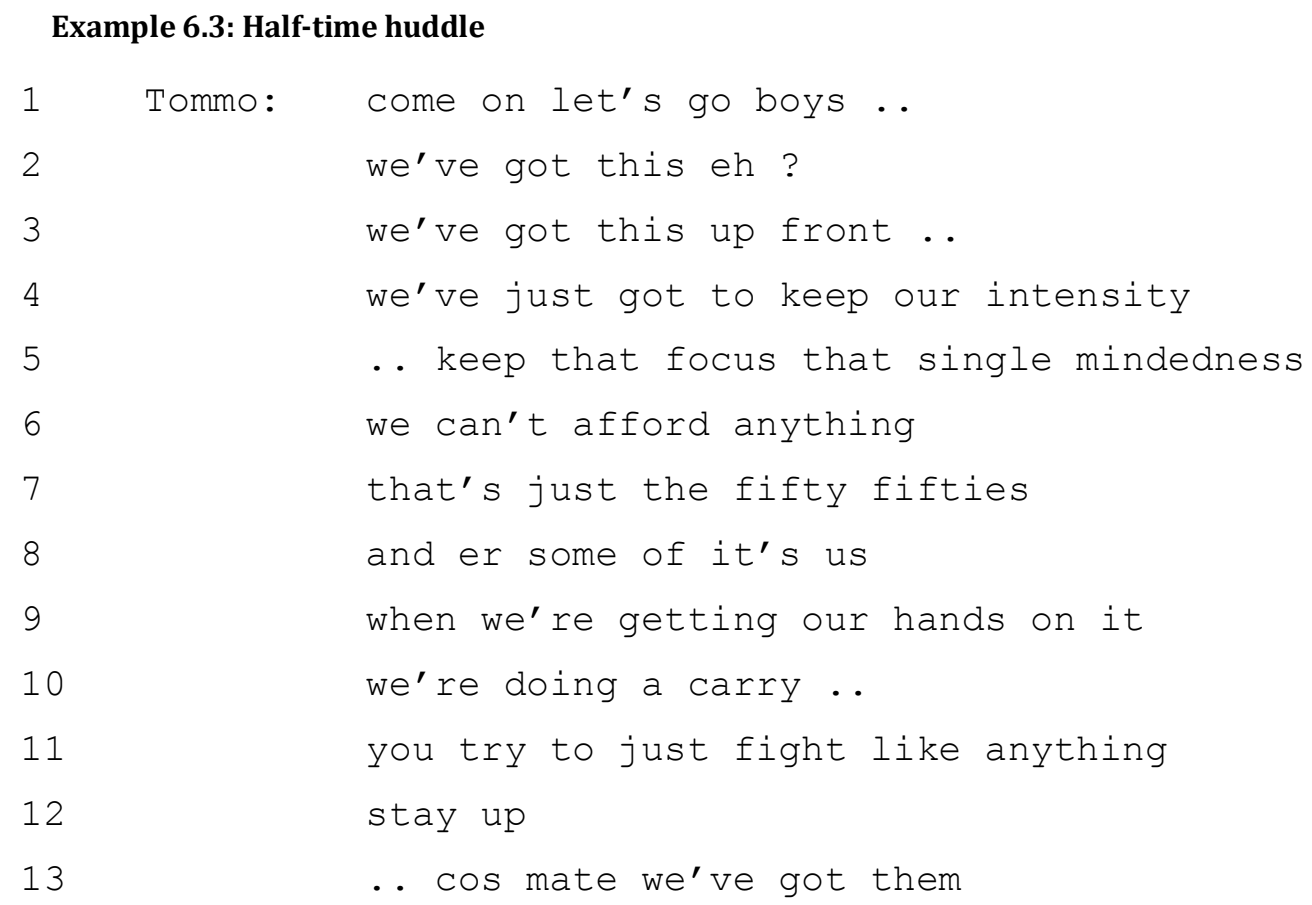


if we stay up off the ground ..

it's the only place they're beating us

cos they're sending more numbers straight

and they're flopping in ..

and let's keep it up..

let's get the drives going

cos if we've got them at scrum time

we'll outdrive them

.. we'll outdrive them at lineouts

and we'll out- we'll out-

we'll punish them at scrum time..

but we just got to keep going eh ?

. stay hungry ..

if you start flagging stick a hand up eh ?

.. but there should-

no one here no one here's fucked eh ?

everyone wants more

Steve: fucking eh

Tommo: =boys enjoy this=

Jon: boys ...= this is some fucking awesome

rugby you tighties are playing eh ?

and you loosies...

fucking hell at the .. scrum time

we've fucking got the acid on eh ?

Ata you're fucking destroying Jamie on the

=fucking on the left hand side=

Tommo: =you are mate . . you're wrecking it=

Jon:

right if fucking we bust over and it's

their ball Cammy .. fucking Moz

I want you to fucking smoke that number

eight eh ?

I want to see him on the ground and us

blowing the fuck over him right?

Tommo: =bang him .. bang him hard=

Jon: $\quad=\ldots$ he $=$ doesn't get a fucking (answer) ok

..we got to be ruthless now boys..

fucking ruthless 


\section{N. A. Wilson}

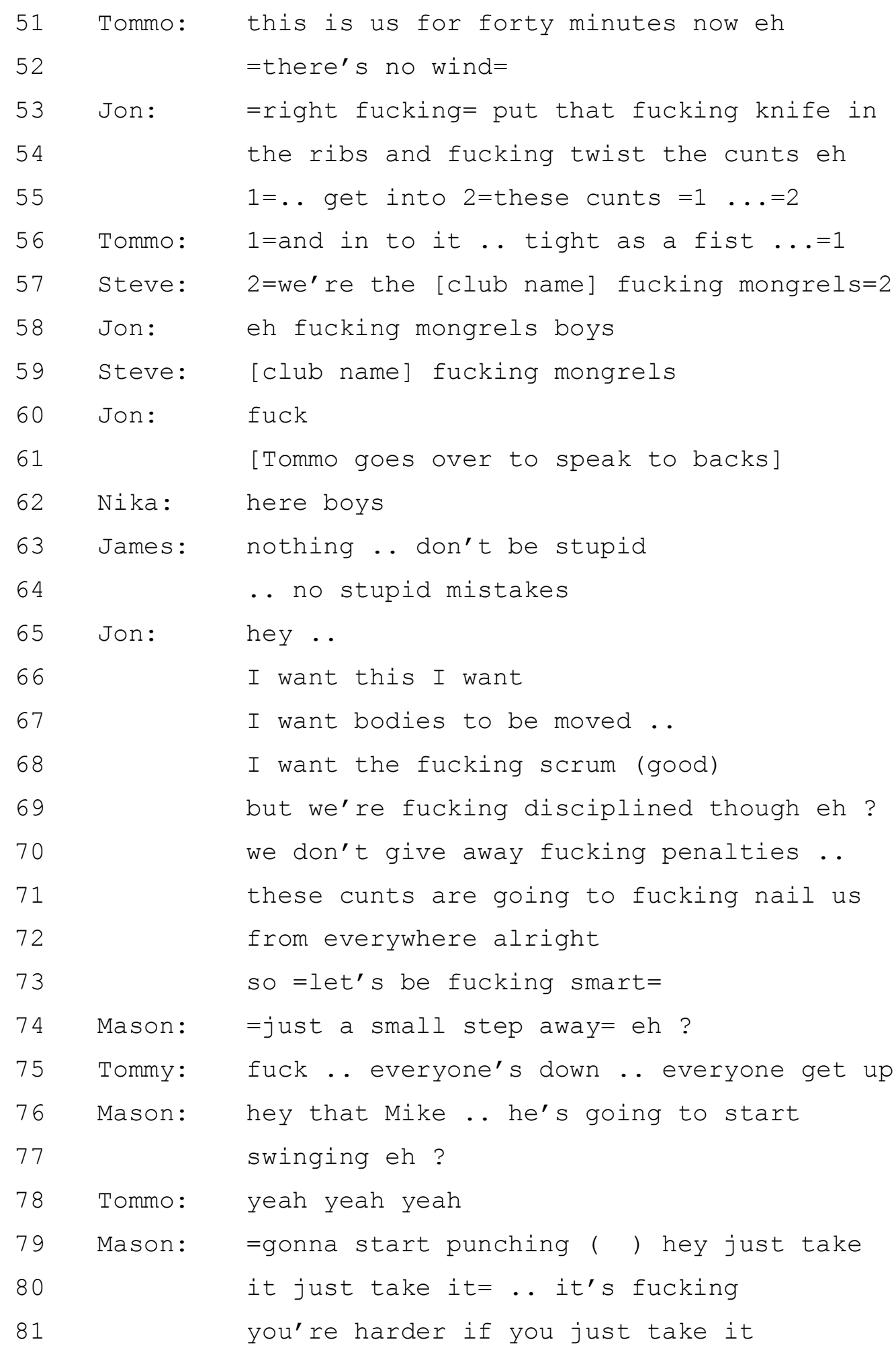

Tommo establishes a team identity that indexes controlled aggression in his directives to the Forwards to fight like anything (line 11), and punish them at scrum time (line 24). He also indexes toughness, by giving the option for players to volunteer for substitution (lines 27 \& 28), but then suggesting that no one here no one here's fucked (line 29), thus 
implying that any player that does volunteer to be substituted does not live up to his expectations. As shown in the previous chapter, leaders have considerable influence on the norms of behaviour within the team and positional CofPs and by communicating his expectations of the players in this way Tommo effectively defines what might be considered appropriate behaviour regarding responses to injury and fatigue in the team. By indexing behaviour that is associated with masculinity and rugby ideology Tommo is not creating a new expectation, but simply reinforcing a norm of behaviour that draws on the players' existing experiences of playing rugby and performing the identity of men that play rugby. These norms and expectations were further evident in the narratives that injured players constructed about their injuries ${ }^{21}$. They would downplay the seriousness of the injury, and stress their desire to play and their frustration with being out of the team. However, injured players would continue to attend training in order to engage with the team and maintain their place in current team practice. The injured players may be seen as pushed to the periphery of the team by their non-participation in the same activities as the other players. However, by continuing to be present at training sessions, even if it is on the sidelines, they stabilise their outward trajectory so that they are more quickly able to regain their former place in the CofP on their return. Not only was this noted by me as an observer, but it is known to the players, especially those who are core members of the CofPs, as Bug attested in interview:

\section{Example 6.4: Interview with Bug}

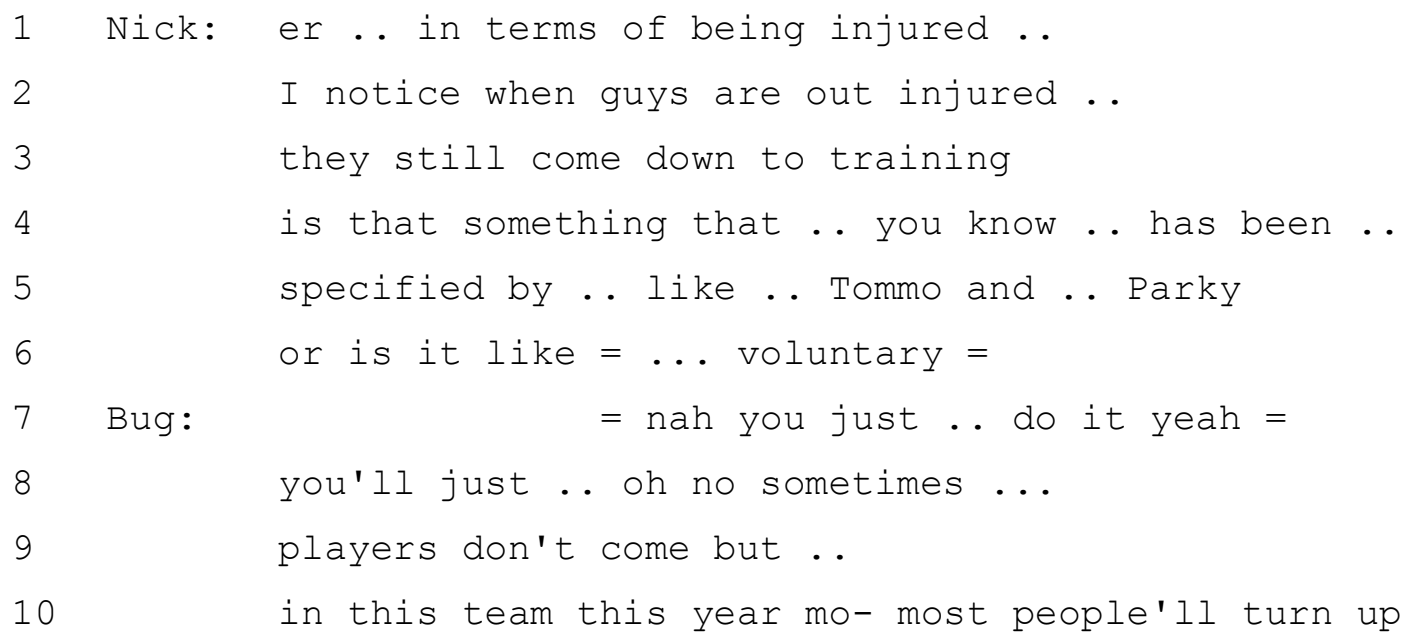

21 Unfortunately none of these were captured on audio recording clearly enough to be transcribed. 


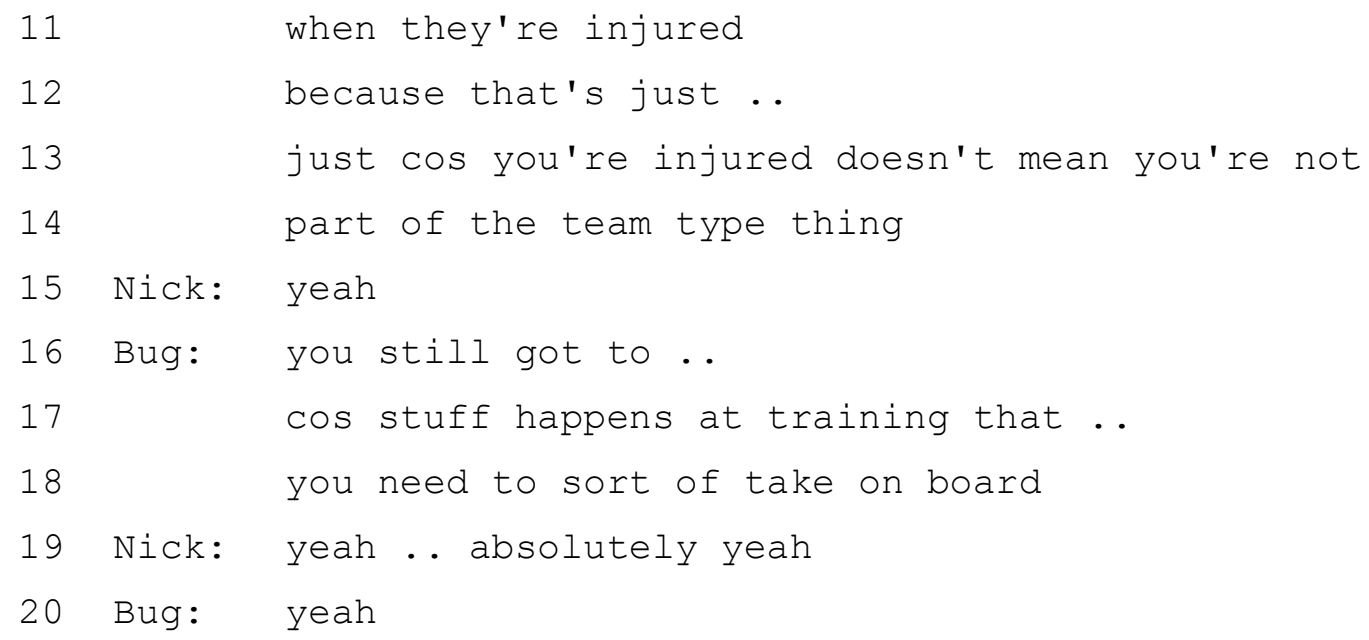

As can be seen from Bug's explanation of why players continue to attend training when injured (although they do not physically take part), players continue to attend because stuff happens at training that you need to sort of take on board (lines 17 \& 18). What Bug refers to is that by missing out on training, injured players no longer have a role in the team. He does, however, indicate that this is a practice that happens in the team this year (line 10), implying that he sees this as an important practice of the Prems' in the current season, perhaps differentiating them from past seasons' Prems or from other teams in the club such as the Senior Ones. As a result, players that are injured are able to renegotiate their own identity within the team, just as Bug did following his own injury later in the season (as described in Chapter 5). Through continued engagement with the team, players can prevent themselves being pushed to the margin of the CofPs of which they are members.

Returning to the analysis of Example 6.3, it can be seen that while Tommo indexes the controlled aspect of aggression and violence, Jon is less circumspect in his use of violent imagery in his words to the Forwards. He specifies particular players that he wants targeted (lines 43-46) and uses powerful metaphors of violence to raise the aggression levels of the Forwards (lines 53-55). Each of Jon's directives however is supported by Tommo, using supportive overlapping speech (lines $47 \& 56$ ) which seems to endorse what Jon is asking of the players. As has been shown in Chapters 4 and 5, this is a discourse strategy that is an important part of the Forwards' discursive practice, and thus very much a part of being a Forward. Jon also indexes the need for control, perhaps more explicitly that Tommo, saying I want the fucking scrum (good) but we're fucking 
disciplined though (lines 68 \& 69). This may be an attempt to soften the illocutionary force of his earlier exhortations to violence and bring his leadership stance into line with Tommo's, a stance which is echoed in Mason's advice addressed to the Forwards in which he tells them not to react if an opposition player tries to start a fight, telling them, you're harder if you just take it (line 81). Mason's comment thus indexes both control of aggression and toughness (tolerance of pain and injury). Between these three speakers, all of whom are members of the Forwards CofP and the Leadership CofP, an identity for the Forwards is created that indexes the (stereotypically) masculine themes of control, violence and toughness. These themes can be found in much of the sports masculinity literature (e.g. Anderson 2009; Iacuone 2005; Messner 1995; Nauright and Chandler 1996). However there are others not found in the discourse of the Prems, such as homophobia and overt sexism which, as shown below, are widely described as aspects of masculine identity, particularly in sport.

Although much of the early research on gender identity in sociolinguistics has been concerned with the way in which men and women's language either reinforces (e.g. Bailey and Timm 1976; Holmes 1986a; Lakoff 1973; Oliver and Rubin 1975; Tannen 1990) or contravenes gendered stereotypes (Cameron 1997), more recent research views gender identity as more fluid, with individuals able to construct an identity that uses both "masculine" and "feminine" discourse features (e.g. Corder 2004; Eckert and McConnell-Ginet 1992; Hall and Bucholtz 1995; Holmes 2006; Mullany 2010). Much of this research has focused primarily on women, and for good reason, as women's language had effectively been sidelined by much of the academic establishment for hundreds of years. However, the concepts arising from feminist investigations into linguistic gender identity are also highly applicable to the study of men's language (e.g. Clayton and Humberstone 2006; Coates 2003; Holmes 2009; Kiesling 2007; De Klerk 1997).

In the previous chapter, mention was made of gendered leadership styles, with relational leadership often being seen as a normatively feminine style. While stereotypes abound, and are often used as the starting point for research in gender identity, many scholars agree that gender is a relational construct (e.g. Cameron 2008; Holmes and Stubbe 1997; Kiesling 2005; MacLean 1999; Meân 2001; Tagg 2008), and that an individual may create their gender identity differently dependent on the people 


\section{N. A. Wilson}

they interact with and the social context of the interaction. This is not to say that stereotypes are not useful in analysing gender. In fact as stereotypes are often indexed in the creation of gendered identity, they are a very good starting point. Moreover, a person's gender identity is created through the interactions in which they participate. Gender identity is not necessarily correlated to biological sex (Bucholtz and Hall 2004; Connell 1987, 2005) but is constructed through the stance an individual takes in their interactions with others. Gender identity is also not as straight-forward as "masculine and feminine". Gender could instead be seen as a continuum with hyper-masculine at one end and hyper-feminine on the other and the vast majority of people constructing their gender identity somewhere in between (Holmes and Stubbe 2003b). Despite the view that gender operates independently of sex, most research that deals with gender does in fact use sex to categorise the participants. Studies on masculinity are still (for the most part) carried out on all-male groups, and thus the discussion of masculinity typically focuses on the gender identities of men. These gender identities are of course many and varied and like any other identity are dynamically recreated according to context. In other words a man may adopt a stance that positions him as "more masculine" when in a sports team full of men, yet when in a mixed sex group may instead adopt a gender neutral or perhaps more feminine stance through the range of discourse features that he employs. This difference has been noted particularly in research on swearing (e.g. Bayard and Krishnayya 2001; Jay and Janschewitz 2008; De Klerk 1997; Limbrick 1991; Staley 1978; Stapleton 2003).

Kiesling, in summarising the research on male discourse strategies, states that it has "generally show [n] men as less polite and more competitive than women, although there is wide variation among men" (1996:667). Kiesling's own research focuses on how men interact in homosocial groups, in particular college fraternities, and how they use politeness and varying usage of vernacular features to both exercise power and to engender solidarity (Kiesling 1996, 1997, 2001a, 2005, 2006). They also compete in terms of turn-taking and interruption. Furthermore, Kiesling (2005) points out that one of the functions of the discourse in these groups of predominantly white, heterosexual men is to maintain relationships within the group while constructing a heterosexual identity. 
Similarly, Cameron (1997) shows that in groups of white heterosexual men in the United States, men position themselves as heterosexual by gossiping about other men and constructing them as gay (regardless of whether or not they are). Cameron shows that the men use the stereotypically feminine discursive practices of gossip and cooperation, to create masculine identities that are very much in line with hegemonic masculinity.

Masculinities have been the focus of a considerable amount of sociological research, particularly in relation to sport (e.g. Adams et al. 2010; Anderson 2008; Bryson 1987; Clayton and Humberstone 2006; Curry 1991; Dunning 1986; Messner 1995; Tagg 2008; Wellard 2002). While there are few explorations of the sociolinguistic construction of gender identity in sport (e.g. Halone and Meân 2010; Kuiper 1991; Meân 2001), sociological research that has been carried out suggests that the socio-cultural norms embedded in many sports seem to perpetuate hegemonic masculinity and the positioning of sport (in particular team ball sports), as games for men who perform their masculinity along orthodox lines (Anderson 2005; Clayton and Humberstone 2006; Sheard and Dunning 1973; Tagg 2008). In other words, the stereotypical sporting male is tough and in-control, but also must demonstrate that they are not feminine or homosexual. In much of the literature this is shown through the misogynism (Schacht 1996) and homophobia (Anderson 2005; Tagg 2008) of sportsmen, and these aspects of the stereotype are easily identifiable in discourse (e.g. Clayton and Humberstone 2006). Most of the research exemplifying this may be regarded somewhat dated, and although it may be viewed as optimistic, some recent research shows that sports player discourse is becoming less sexist and less homophobic (Adams et al. 2010; Anderson and McGuire 2010). However, the ideals of toughness and violence are still evident in the discourse of sports players, as this section shows. They are indexed directly in the downplaying of injury seriousness (Fenton and Pitter 2010; Howe 2001) and the violent imagery used in huddles (e.g. Examples 6.3 \& 7.3). Indirectly however, these aspects of sports masculinity are indexed through discourse features such as high frequency swearing, jocular insults and unmitigated FTAs, all features stereotypically associated with all male groups (Bayard and Krishnayya 2001; Kiesling 1997). However, these features are also used in the creation of solidarity (Daly et al. 2004; Hay 1994; Holmes 2009), so it 


\section{N. A. Wilson}

may be that there is a cross-over between masculine identity creation and solidarity, or perhaps these ways of engendering solidarity are linked to masculine stereotypes.

Interestingly, research that has been carried out on female sports teams has shown that women playing in competitive sports teams adopt a discourse stance that indexes the male sporting stereotype, while players in social teams do not (Corder 2004). This begs the question of whether the identity being indexed is in fact masculine. It may be that the identity that is indexed is that of the competitive sports player and that this identity is linked to a masculine stereotype (Kuiper 1991). Thus the masculine stereotype that pervades sporting discourse is in fact layered so that in indexing a sports identity one also indexes another layer of identity in the ideology of orthodox masculinity. As noted above, there is almost an entire sub-discipline of sociology devoted to analysing the myriad constructions of masculinity as it is related to sport and it is for this reason that it is mentioned here. However, in order to retain focus on the way in which language is used to index the form of masculinity commonly constructed in rugby, it would not be accurate to say that a rugby player constructed a particular type of masculinity in their speech (in the rugby team), rather that they were taking an epistemic stance towards the ideology of masculinity that is embedded in existing New Zealand rugby discourse. This ideology has been constructed primarily through nostalgic accounts of tough, heroic, male rugby players and their role in New Zealand's colonial past (Hughes 2005; MacLean 1999; Phillips 1996; Ryan 2005a). Therefore, when I mention "sports masculinity", it is the stereotype of the tough, disciplined player with undertones of misogyny and homophobia that I refer. I do not claim that the players in the Prems construct this masculinity, but that in their use of indexical markers they construct a masculinity in their relative stance to this ideology.

The indirect indices of sports masculinity that are found in the literature are frequent swearing, insults and unmitigated FTAs. As Example 6.3 illustrates, the directives and criticism addressed by Tommo, Jon and Mason to the rest of the Forwards are all mitigated, whether it be by hedging using (discourse marker) minimisers like just (lines $4,7 \& 11$ ) solidarity markers such as the inclusive pronoun we (lines 4 \& 14), familiarisers like mate (line 13) or the juxtaposition of directives with positive appraisals of the Forwards' first half performance such as we've got this eh ? we've got this up front .. we've just got to keep our intensity (lines 2-4). 
As described in Chapters 3 and 4, the function of the half time huddle is for the coaches and captain(s) to provide criticism, encouragement and to direct the players in what to do in the second half. Their speech contains a mixture of compliments, criticisms and directives in order to fulfil the functional requirements of the communicative events. Furthermore, the identity of the speakers as members of the Leadership CofP and core members of the Forwards means that any potential interpretation of either criticism or directives as face threatening is mitigated through the fact that their behaviour is highly appropriate and expected by the players in this communicative event. In other words the use of these speech acts in the half time huddle indexes local leadership stances, which index a coaching register (Heath \& Langman 1994; Kuiper \& Lodge 2004; Masterson et al. 2006) that is associated with masculinity (Anderson 2007).

There are also no intra-team jocular insults present in this extract. However there are insults directed at the opposition, as noted in Chapter 5 , with Jon referring to the opposition as cunts (lines $54,55 \& 71$ ). As has been mentioned previously this serves to create an us/them distinction between the Prems and their opposition. This leads to one particular index of rugby identity and masculinity that is present in almost every communicative event that takes place with the Prems, swearing.

In preceding chapters, swearing has been identified as being a feature of the teams' shared repertoire; it is entirely appropriate in the norms of the team to swear frequently. However, in addition, the frequent swearing also indexes a masculine identity. It is men who are stereotypically thought to swear more than women and use stronger swear words. Many early studies have indeed shown this (e.g. Bailey and Timm 1976; Nykodym and Boyd 1975; Oliver and Rubin 1975; Staley 1978), although more recently and following a CofP approach, it has been shown that women and men can swear to the same degree when in homosocial groups (Bayard and Krishnayya 2001; Daly et al. 2004; Stapleton 2003). However the stereotype does have currency when it comes to the identity of male rugby players. As far back as 1882 it was noted that "the frequent use of oaths and foul language by some of the players during the excitement of the game [rendered] it unsafe for ladies and children to be present at such contests" (Phillips 1996:77). As the data in this research shows, little has changed in the frequency of "foul language", except that the swearing is generally confined to the team room and the huddle. Certainly in current times rugby players swearing within earshot 
of the referee can be penalised and anyone, supporters included, can be banned from rugby for swearing directly at the referee. Of particular note in Example 6.3, is Jon's use of cunts (lines $54,55 \& 72$ ) to refer to the opposition. While the other players certainly swear, as can be seen from the numerous occurrences of fucking as an emphatic intensifier, Jon was noted as a prolific swearer even among the players. This extract, in fact, was used when conducting a feedback session with the players the following season, and one of the first comments from a player was does he really need to swear so much? Although the frequency of Jon's swearing may have caused comment when the interaction was listened to in the feedback session, and where it was met with laughter, at the time it was taken as deadly serious. The swearing, even the forceful swearing, is appropriate for the half-time huddle more than at any other time, because it is vital that the players do not lose their emotional charge. The use of swear words to "other" the opposition helps to create a boundary between the Prems and the opposition. However, the frequency also conveys the spontaneity of Jon's speech, as fucking is often used as a filler, as shown in lines 41 and 42 (reproduced below). These contrast with the use of fucking on line 43, which functions as an adverbial intensifier. That is not to say that using fucking as a filler does not affect the illocutionary force of a statement, quite the contrary, it mostly functions as an intensifier.

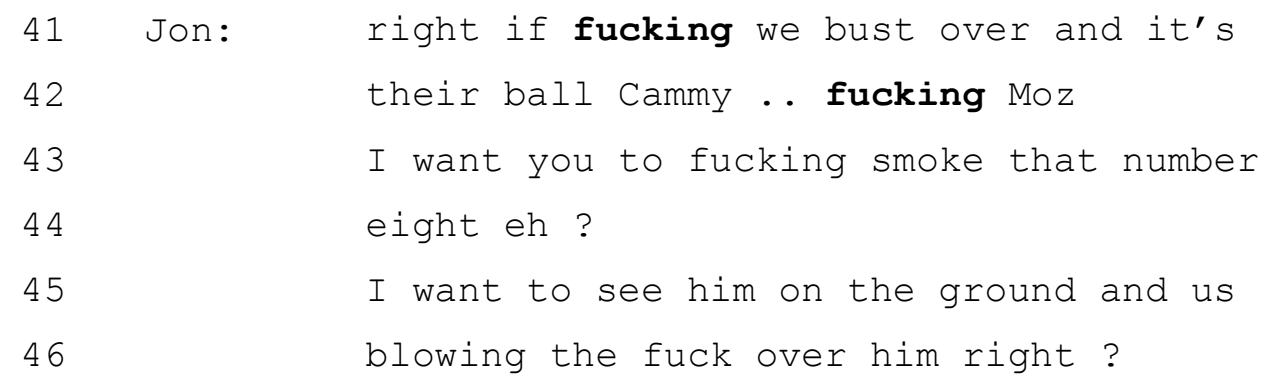

The high frequency of swearing by all the players (some more than others) indexes multiple, layered identities of which sports masculinity is but one. As discussed in previous chapters, it is part of the repertoire of communicative practice that defines the team CofP. It also indexes the socio-cultural identity of rugby, because swearing is clearly a part of the rugby player stereotype, as mentioned above. Through this masculinity is indexed. Thus the use of frequent swearing may be analysed as indexing a stance of high emotional involvement or solidarity as its first order of indexicality, CofP membership as its second, rugby player identity as a third, and masculinity as a fourth 
order of indexicality. As Silverstein (2003) suggests, this could conceivably occur to the degree of $n+1$. In other words, there is no end to the number of identities that could be indexed by a communicative practice such as swearing and of course, the identities that are indexed are dependent on the socio-cultural knowledge of the hearer as much (if not more so) than the speaker (Ochs 1990).

\subsection{Locally negotiated masculinities}

Having examined the way in which masculinity is indexed through stances promoting rugby ideologies, this section considers how these masculinities are renegotiated within the team. Unlike the indexicalities present in front-stage communicative events such as huddles and team meetings, the local negotiation of masculinity appears to take place more often in back-stage encounters where players are not constrained by the particular situational norms that contribute to the character of front-stage events. One of the ways in which this negotiation takes place is through the discourse strategies developed by each CofP within the team and the negotiation of power between individual players.

Example 6.3 has shown that a non-masculine, supportive discourse strategy is used in the Prems, particularly among the Forwards. However, as pointed out in Chapter 4, the Backs often adopt a more stereotypically masculine, competitive discourse strategy. Competitive discourse is identifiable by disagreement, as noted in the analysis of Example 4.2, an excerpt of which forms Example 6.5 below. Example 6.5 however, isolates a particular part of Example 4.2 relevant to masculinity, the use of jocular insults in creating gendered identities. While the use of jocular insults may be analysed as indexing rugby stereotypes, the way in which these insults are deployed and their integration into negotiations of power is a locally constructed phenomenon. Hence, while ideologies and stereotypes may be indexed as third order indexicalities, the negotiation of different masculinities between the players is a stance that renegotiates the more global indexicalities such as masculinity stereotypes contained in rugby ideology. Once negotiated these become local masculinity identities. Indexing them can be regarded as second, rather than third, order indexicality. Furthermore the gender identities that are indexed in back-stage events are not necessarily drawn from rugby ideology. 


\section{N. A. Wilson}

Example 6.5: You bitch

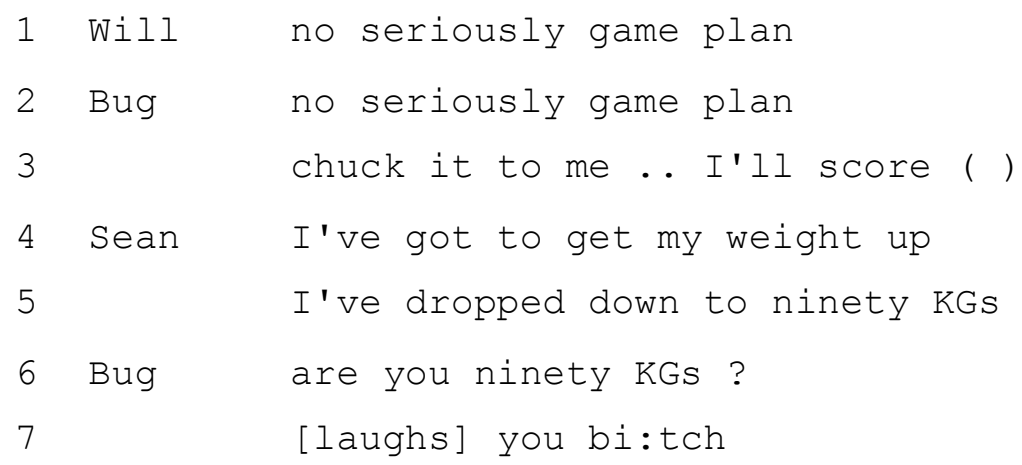

In calling Sean a bitch (line 7), Bug implies that by complaining about his weight, Sean is indexing a stereotypically feminine identity. Furthermore, ninety kilograms is very light for a rugby player and thus Bug's comment may be interpreted as indicating that Sean's lack of weight (and by inference muscle) makes him less masculine. However, his laughter prior to the comment indicates that it is not meant to be taken seriously and his intonation of you bitch indexes an exaggeratedly effeminate speaking style, further contributing to the idea that while used in a jocular fashion, Bug is essentially challenging Sean's masculinity (Adams et al. 2010).

Challenging other players' masculinity is a strategy that Bug uses on other occasions to assert his authority as a high status member of the team based on his institutionally reified membership of the Leadership CofP (see Chapter 5). This is shown in Example 6.6 below, in which Bug indirectly challenges Will's masculinity by questioning his skill at kicking, a key skill for both Bug and Will's positions. This challenges Will's masculinity because this skill is based on control, thus suggesting that Will lacks control over his actions and implies that he is less masculine than Bug. Furthermore, in this example both Bug and Will index masculinity through the high level of disagreement that they exhibit with each other (Holmes 2009; Kiesling 2001b). As stated above this is a feature which is also consistent with the Backs' discursive repertoire.

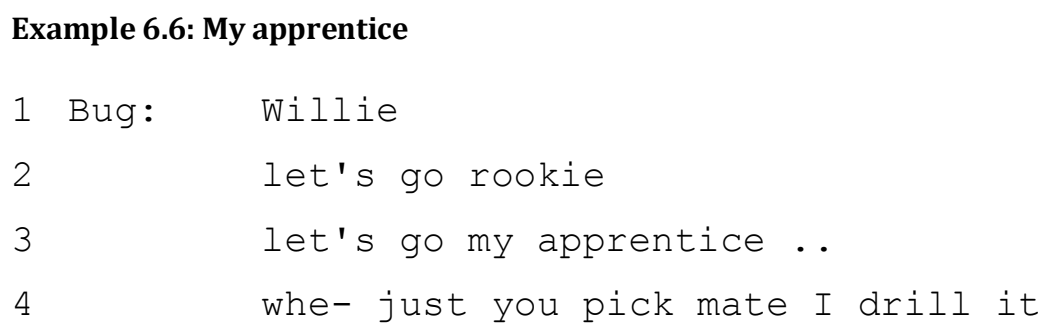




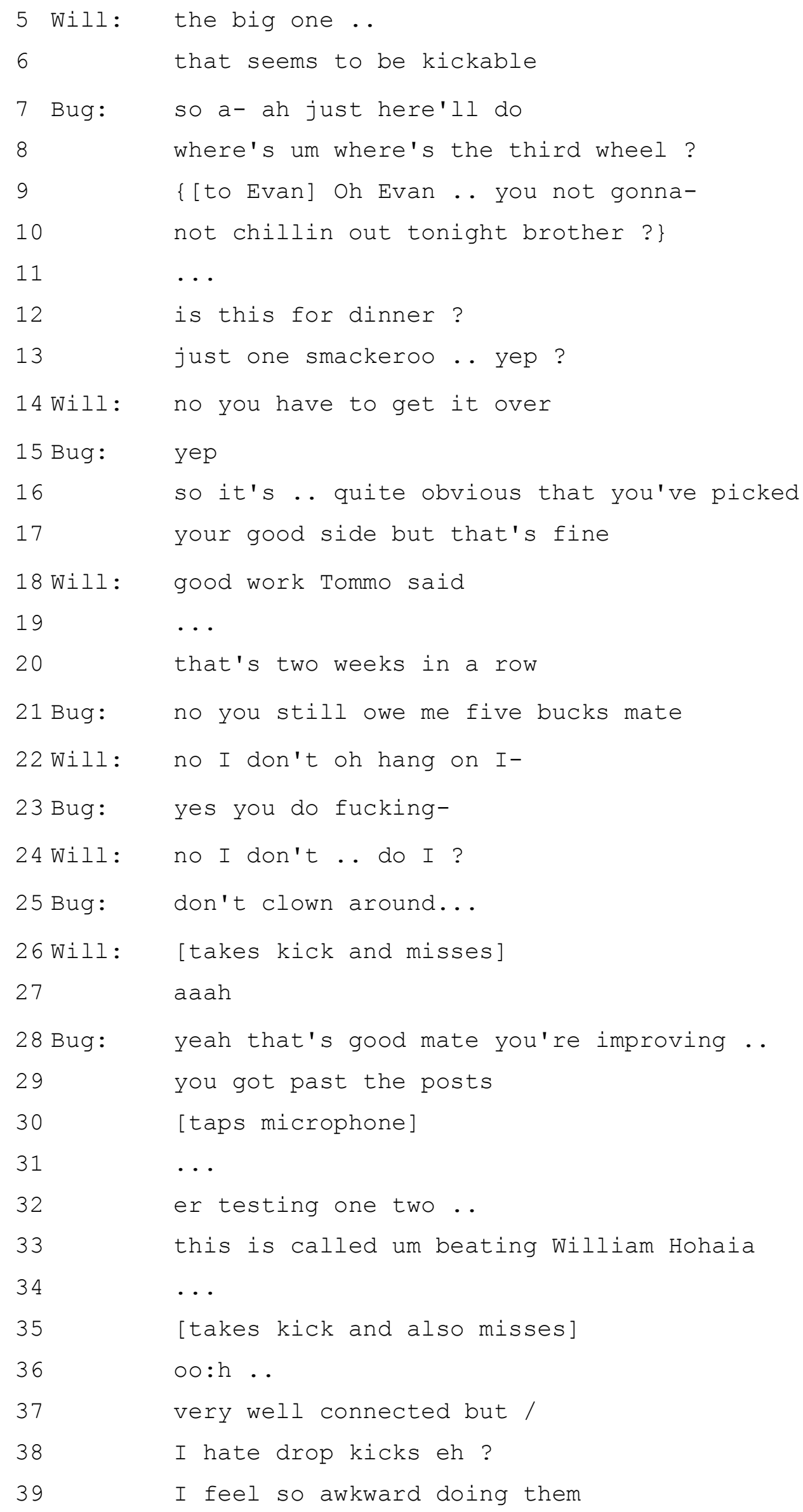




\section{N. A. Wilson}

There are a range of ways in which Bug constructs what may be viewed as hegemonic masculinity in this interaction. He takes a stance which places him in a position of power over Will, based on his experience as a player, closeness to the core in both Backs and Leadership CofPs and age. All of these aspects of identity are indexed through Bug's construction of the master/apprentice relationship between the two players (lines 2, 3 \& 28), but these same issues of power also index masculinity (Kiesling 1997). In effect Bug positions Will as of lesser status than him, but Will challenges this by taking up an equally competitive stance, disagreeing with Bug on both the rules of the kicking contest and whether or not he owes Bug money (lines $14 \& 22$ ). Through the interaction, which is after all a competitive encounter, each player attempts to gain the upper hand in deciding what they are doing (c.f. Example 4.2). Bug asserts his dominance by trying to reset the challenge to be about how far each could kick (line 13), despite Will having already suggested a target (line 5). Will rejects this however and makes it clear that the ball needs to go over the goal (line 14). Bug then prepares an excuse in case he misses by suggesting that Will has picked his stronger side and therefore has an advantage (lines 16-17), which Will does not respond to. Instead he tries to establish an identity as a favoured player by referencing an earlier comment from Tommo. Bug does not acknowledge this and instead accuses Will of owing him money. They dispute this until Will grudgingly concedes. (This meaning of $d o I$ ? is conveyed through the uncertainty in Will's tone, a feature difficult to show in the transcript).

Bug's appraisal of Will's kick, while appearing at first to be a compliment, sounds quite sarcastic in tone, and again positions Will as the apprentice. Bug's own kick also misses, but he both compliments himself (line 37) and makes an excuse for his performance. Lines 30-34 have been seen before in Chapter 3 and are interesting in that they show Bug using the recording device that he is wearing as a prop (and audience) for asserting power. It is unclear who Bug thinks he is addressing here; does he merely want to state his intended outcome for the record or does he want to remind Will that this is being recorded in order to increase the stakes of the challenge (as with his earlier audience seeking interaction with Evan at Lines 9 \& 10)? Whatever his motivation, his speech takes on a more formal style, using Will's full name and mimicking a sound check, a clear example of referee design (Bell 1984), even if it isn't exactly clear who the referee 
might be. This demonstrates remarkable adaptability and is also a sign of how comfortable the players were with being recorded at this stage in the fieldwork.

Bug and Will's competitive discourse characterises the discursive behaviour of the Backs, where group identity is concerned with one-upmanship and competition, both indices of masculinity (Frohlick 2005; Iacuone 2005; Thompson and Whearty 2004). Although both Forwards and Backs used jocular abuse to a certain extent, in the encounters that I observed, the Backs always seemed to be more abusive and the Forwards more jocular. This may simply have been because when insults occurred among the Backs the speaker laughed, while among the Forwards everybody laughed.

Despite a difference in discourse style and stance, the ideologies that are indexed through the content of both Forwards and Backs discourse construct masculine identities that are in line with some rugby ideology. That the discourse strategies used by each CofP do not match with the masculine sports stereotype is perhaps an indication that, as Holmes suggests (2006), these strategies are not inherently masculine or feminine but are developed in response to the contextual needs of the situation in which different CofPs operate. This is not to say that the Prems are not creating a form of masculinity in using them, rather it is a masculinity that is appropriate to the needs of the Prems and the CofPs within.

\subsection{Other macro-identities}

Although the macro level of identity that this chapter has focused on is masculinity, there are other macro level identities that are indexed through the team's discourse. One is age, another is ethnicity. It may be considered that age is indexed directly through many of the features that can be found in the players discourse, such as high frequency swearing, use of $e h$, and a number of other discourse features that are viewed as markers of "young” or "cool” New Zealand English (Bell 2000; c.f. Kiesling 2004). However, age and ethnicity may be indexed in many ways simultaneously and it has been noted that many features of New Zealand English that were hitherto associated with Māori English are now features of the speech many young New Zealanders, regardless of ethnicity (Bell 2000; Holmes 2005c). Thus, among the players it is difficult to say whether certain features, such as eh or bro, index age or ethnicity. Following the 
line of argument that the team construct multiple identities simultaneously the answer could of course be both (Holmes 2005c).

Chapter 5 showed how Tommo takes a stance that indexes Māori identity through the use of Māori words such as koro and high frequencies of final tag eh. It was suggested that these features form part of his repertoire gained through his social networks outside of the Prems rather than a claim to Māori identity as such. It is, however, an appropriate stance to take in the Prems as over half of the players lay claim to some form of Māori identity, both linguistically and through their participation in Māori rugby teams. However, many ideologies of rugby may be considered as indexing not just masculinity, but (in New Zealand terms) Pākehā masculinity, with the norms of interaction that are enshrined in the rituals of the rugby club drawing from Pākehā, rather than Māori or Pasifika norms (Grainger 2009; Hokowhitu 2003; MacLean 1999; Melnick and Thomson 1996; Palmer and Masters 2010). Consequently, stances that construct an affiliation to an ethnic identity use discourse features that do not index rugby ideologies, but directly index ethnicity. This constructs an identity that could be regarded as relevant to the Prems as a group of young New Zealand males rather than as rugby players (Stubbe and Holmes 2000). Of course, this can occur simultaneously with all of the other levels of indexicality that have been mentioned.

The difference between the discursive practices of the Forwards and Backs CofPs could also be analysed in terms of ethnicity. The Forwards' supportive and at times selfeffacing style could be interpreted as conforming to Māori norms of interaction while the Backs' more competitive style and displays of self-aggrandisement might be seen as indexing Pākehā norms. Whether the differences in communicative practice between Forwards and Backs are influenced by this, masculinity, or spatial organisation, or all three, is one of the topics discussed in Chapter 7.

\subsection{Summary}

This chapter has examined levels of identity beyond those that are situated in local practice and discussed the way that these are negotiated into a locally constructed team identity. Using the concepts of indexicality orders, a set of ideologies drawn from the socio-cultural context of rugby have been identified in the discourse of the Prems. However these are not indexed directly, but by means of stances that develop with 
reference to local norms and behaviours that form the repertoire of the CofPs in the Prems. Some of these ideologies index another level of identity: masculinity. However, it has been shown that while stances taken in front-stage communicative events in the Prems index masculinity by way of rugby ideology, those that are adopted in back-stage events index masculinity more directly. Furthermore the stances that are enacted by individual players not only contribute to the negotiation of team identity, but simultaneously construct multiple identities for the team that can be used as a resource in adopting stances that further the organisational goal of the team, which is to win matches.

The rugby ideologies used in constructing the Prems' identities have been categorised into territorial ideologies and masculinity ideologies and examples have been analysed to show how these ideologies are referenced directly and indirectly through the stances that players and coaches take in a variety of communicative events that involve the team. Additionally the more direct indexing of macro identities such as masculinity, ethnicity and age has been discussed with reference to discourse features and strategies that appear to index multiple identities simultaneously.

This chapter has argued that any description of team identity must include all of the layers of identity and ideology that are indexed, and account for the fact that different identities are relevant to, and constructed by, different communicative events. Furthermore identity changes over time, through the cumulation of stances that are taken by team members as they negotiate a trajectory within the team CofP. Therefore, one cannot say "this is team identity" as it is both layered and dynamic. However, what can be said is that team identity in the Prems is constructed through the local renegotiation of identities and ideologies that are indexed through stance. Team identity draws on the socio-cultural context of rugby and is shaped by the goals and needs of the team as a community of practice and the communicative norms that are negotiated through social practice. 


\section{Chapter 7 Temporality: The renegotiation of leadership and identity}

This chapter discusses how leadership practice and identity construction are integrated within the CofP framework, through the analysis of temporally situated discourse. Temporality, or the way in which time is part of context, can be expressed through mimetic discourse such as formulaic language and ritual communicative events (Allan 2010; Griffin 1992; Schiffrin 2009). In other words, without a temporal dimension to contextual analysis, any discourse involving mimesis cannot be fully described. Framing discourse temporally, as well as spatially, captures the dynamic nature of negotiated communicative practice as the underlying concept that unites leadership and identity in the Prems. Indeed negotiation itself, the cornerstone of the CofP framework, may be viewed as fertile mimesis (Pennycook 2010) because each act of negotiation is in part a repetition of what has gone before and a change, which adds to the repertoire of communicative practices that define a CofP and hence shapes its identity.

The most obvious way in which temporality is apparent in the Prems' discourse is through repetition. This is not restricted to the level of the utterance, however, but can be expanded to encompass the repetition of discourse strategies and communicative events, and even to the reiterative cycle of seasons and the emergence of team CofPs. Thus repetition may be regarded as a sign of temporality at multiple levels of discourse (Blommaert 2005). For instance, the repetition of words and phrases within the bounds of a communicative event can itself become a practice. This has been discussed in reference to lineout communication (Chapter 4) where the Forwards were shown to use repetition as both a relational and transactional discourse strategy, repeating taskoriented speech (such as codes signifying who was to catch the ball) as well as compliments and commentary on what is happening. Over time this can become formulaic, becoming not just a practice available for doing the lineout, but (linguistically speaking) how the lineout is done. Using formulaic structures of language, other communicative events, particularly those enacted by leaders on match days, recur at the same time and in the same place every week, and thus become ritualised in the creation of the Prems identity. Thus it may be seen that repetition and the recurrence of 
communicative events are ways of performing and renegotiating leadership practice and team identity that can only be analysed by viewing practice and identity (via stance) in relation to time.

The temporal link between practice and identity is not a new concept, as Wenger's quote below illustrates.

The temporal dimension of identity is critical. Not only do we keep negotiating our identities, but they place our engagement in practice in this temporal context. We are always, and at the same time, dealing with specific situations, participating in the histories of certain practices, and involved in becoming certain persons. As trajectories, our identities incorporate the past and the future in the very process of negotiating the present. (Wenger 1998a:164)

In other words, identity is a record of the way in which different stances are taken over time (Jaffe 2009). Wenger uses the concept of trajectory to describe identity in terms of an individual's relative position to the core of a CofP over time. This is derived from the original focus of the CofP model as a description of situated learning (Brown and Duguid 1991; Lave and Wenger 1991). While this is an intrinsic aspect of the CofP model (through learning linguistic behaviour we display group (CofP) affiliation) there are further directions in which the CofP model can be taken. In the case of this research, the idea of the negotiated linguistic repertoire as a store of identity is viewed as an important part of the model. This has been influential in theorising leadership as a distinct practice around which a further CofP forms rather than viewing leadership as brokerage of practices between CofPs (see Chapter 4). It has been shown that in the Prems leaders tend to be core members of positional CofPs, and that the leaders have negotiated not only a way of enacting leadership but of talking about it. This idea has thus arisen from the data. Practices such as the use of a particular leadership stance in a particular communicative event form the content of this repertoire of leadership practice and are negotiated through the constant repetition of events inherent in the way that rugby matches and training operate (Chapter 5). The two aspects of mimetic 
behaviour that can be identified as important in constructing leadership practice and team identity in the Prems are ritual and formulaic language:

Ritual: the mimetic re-enactment of a particular communicative event with reference to its place in space and time (Goffman 1967; MacLean 2004; Sarangi 2004). For instance the re-enactment of the pre-match huddle at home and away matches becomes ritual through mimesis of temporal and spatial positioning (Chapters $3 \& 6$ ). In addition ritual contains a symbolic element. In the case of the ritual events in the Prems, they index rugby ideologies and a global culture of rugby.

Formulaic language: the repetition of phrases and neologisms across communicative events. While these may draw on outside sources, such as rugby ideology, they may also be locally constructed, such as the brothers call that is used to mark the end of every huddle. It is not simply the repetition of the phrases however, but their function that gives them their formulaic status (Wray and Perkins 2000). Furthermore, this may change through use and re-use; hence as a part of a CofP's repertoire the function of formulaic language may be renegotiated over time. The classic example of this is the way in which the function of formulaic insults is renegotiated in order to mark solidarity (Kuiper 1991). Formulaic language is also closely tied to indexicality, as by using a particular linguistic formula one can index the identities of other people who use that formula, a form of referee design (Bell 1984, 1999). It can also index a shared past event or situation, thereby indexing a discursive style that has local meaning and hence marking a communicative event as having a particular function that is embedded in the socio-cultural knowledge of the community. However, formulaic language refers only to the predictable construction of individual phrasal units, as demonstrated in Section 7.2. To describe the way in which events themselves follow a repeated pattern, the term routine is used to refer to the predictable organisation of utterances within an event. This routine structure can quickly take on ritual significance in that it is one of the identifying features of an event as a ritual, in concert with spatio-temporal location and the participants involved. 
As indicated above, both of these stylistic devices stem from repetition. Repetition can be found at multiple levels of analysis of the discourse of the Prems. There is the microrepetition of formulaic words and phrases within and across communicative events, the formulaic repetition of event discourse structure from match to match, the repeated location of communicative events both spatially and temporally within the match day, and the repetition of the formation, negotiation and dispersal of communities of practice from one season to the next. Thus the three topics of the community of practice, leadership discourse, and team identity will be woven together throughout this chapter, drawing on multiple levels of temporality and repetition. The first section of this chapter discusses the importance of ritual communicative events in terms of creating team identity and a repertoire of practice from which leaders draw to enact leadership. Following this is a discussion of the linguistic formulae that are used within the Prems, how these are used in leadership practice, and their impact on team identity. Finally, the temporally bounded ritual of the entire season is discussed using the final post-match huddle of the season as a focus for the discussion. The analysis of this event demonstrates how formulaic language is used to perform ritual functions in marking the end of the season, while simultaneously indexing the identities of the team and itself being a platform for leadership practice.

\subsection{Ritual and performance}

As discussed in Chapters 3 and 4, there are number of communicative events that take place within the Prems that are repeated in a ritualistic manner, such as the weekly debrief, the team meeting and the various configurations of huddle. Some of these take place during training sessions but the majority are performed at significant times that frame the match day. In fact, it was the very predictability of the occurrence of these match day rituals that facilitated the recording of them for this research. In determining which events are ritualistic we can return to Goffman's dramaturgical metaphor (outlined in Chapter 2 and exemplified in Chapter 5) and view match day events as front-stage and training sessions as back-stage. However, within the training session interaction can be further divided into back-stage and front-stage, just as it can on match days. In training sessions, front-stage may be regarded as interaction involving a group of players and a leader, be they coach or captain, where multiple addressees are addressed at the same time. Back-stage on the other hand could be an interaction 
between two individuals or a group, the distinguishing factor being that back-stage interactions do not include a passive audience. The implication of this is that front-stage and back-stage fit into the framework of audience design (Bell 1984), but with perhaps a greater degree of emphasis on performance and agency. In other words, front-stage interaction is a performance for the benefit of the audience (who may also be participants), the fundamental difference being that at least some of the audience do not make a linguistic contribution to the communicative event, thus positioning them as a passive audience. Furthermore, a front-stage interaction may have auditors, while a back-stage interaction relies upon all of the audience taking part in the interaction and that the back-stage interaction is always in some way relevant to what is performed in the front-stage. Grounded in my analysis of the Prem's discourse, the conceptualisation and application of this metaphor and the use of the terminology goes somewhat further than either Goffman (1959), who used it as an analogy for everyday interaction, or Richards' $(2006,2010)$ application of the terminology which he equates with spatial locations in workplaces.

Therefore, the use of back-stage and front-stage to examine communicative events implies a performative aspect to the discourse that takes place within the Prems. As sports players the Prems perform the core practice around which they congregate in front of an audience, who in turn gather for the purpose of watching. Thus the Prems are performers and rugby as a practice is performative. However, as a sport it is also competitive and thus requires a high degree of adaptability in developing linguistic and physical practices that can achieve the team's shared goal of winning matches. I propose that the front-stage performance of rugby on the pitch is one of the contributory factors to the prevalence of ritual and formulaic language in rugby because the front /backstage division inherent in the match day is repeated in other aspects of the team culture. Hence there is a level of front-stage interaction possible in every gathering of rugby players where discourse is performed so as to put it "on record" (Alexander 2004; Bucholtz 1999; Moore 2006). This viewpoint is evident in research on the language used in other sports (Halone and Meân 2010; Heath and Langman 1994; Kennedy and Zamuner 2006; Kuiper 1996; Kuiper and Lodge 2004) and is one reason why language in sport is a research area that is useful, incorporating into the CofP framework a performative aspect (Alexander 2004; Austin 1975; Hall 1999; Pennycook 2004). This is 
consistent with work by other scholars who use the CofP to reconcile situational and societal constraints on interaction with agency (e.g. Bucholtz 1999; Eckert 2000; Holmes et al. 2011; Moore 2010a) .

Players and coaches rehearse game strategy and physical technique in training sessions but (continuing with the dramaturgical metaphor) they could also be regarded as rehearsing their match day discourse in the negotiation that takes place in training sessions. Consequently, ritual communicative practice is always a front-stage communicative event, whether it is in training or on a match day. Indeed it could be suggested that it is by being performed repeatedly in the front-stage that an act becomes ritual.

Sport is full of ritual; this is one of the reasons why parallels are often drawn between sport and religion (Birrell 1981; Goffman 1967; Goodger 1986). Indeed some scholars suggest that sport is ritual (e.g. Guttmann 1978; Sansone 1988). Ritual communicative events are used to mark the passage of time and to prepare participants for a change in action. In order to demonstrate this, the ritual communicative events that mark the chronology of a match day and prepare players for action are described below. What identifies these communicative events as rituals is that they are events common to all rugby teams, being embedded in the cultural repertoire of rugby. This is the context against which rugby players define themselves, from where ideologies are drawn and renegotiated, and from where a sizeable amount of a player or coach's rugby vocabulary stems (Heath and Langman 1994; Kennedy and Zamuner 2006). By performing the rituals and constructing themselves as part of the rugby culture, the players and coaches also perpetuate it, reshaping what it means to be a rugby player for them through their locally negotiated communicative practices. That a huddle is performed immediately prior to a match is common to all rugby teams, though the specifics of how one performs a huddle will vary from team to team as a negotiable practice. Therefore, the following section, which details which communicative events make up the prematch ritual of the Prems, although describing local practice, has commonalties with other teams and it is the very performance of these rituals that identifies the Prems as a rugby team. 


\subsubsection{Pre-match ritual}

Through the observations made when determining what to record on match days, I discovered that the events that were enacted by the players and coaches followed a strict order and never deviated from this. Furthermore, these events were also spatially bound in that they always were always enacted in the same place when playing at home, while for away matches an equivalent space was created (Wilson forthcoming). The timings for some of the pre-match events are explicitly laid out on posters displayed around the team room over the preceding week. This in itself casts the events as frontstage, reifying the events such as the team meeting through naming them (c.f. Chapter 3 on how the events were chosen for recording). The first event for which a time is specified is strapping, which is where any players who have minor or chronic injuries (as most rugby players do (Howe 2001)) have them bound and padded, or protected in some other way. Around ninety minutes is allocated for this, which gives the players plenty of time to arrive and, more importantly from a linguistic standpoint, gives them the opportunity to engage in small talk, building solidarity from the start (Mirivel and Tracy 2005). During this time the squad managers gather together any equipment that is needed for the match, such as medical supplies, and fill water bottles. They also engage in small talk with both players and coaches. The front-stage of the strapping event can be considered the physio bench where the player is strapped, because this is the defining focus of the event and where players and coaches may move in and out of a performance of injury narratives (Howe 2001). This same physical space, however, becomes an area for back-stage discourse between the squad managers during the team meeting, which is the next event in the chronology of the pre-match.

Officially players are not required to be present for strapping but the negotiated expectation is that all players must be in the team room, seated by the time specified for the team meeting. This was negotiated at the beginning of the season when Tommo rebuked players for not being ready for the meeting, telling them that they may as well take their shit off and go home if they were not prepared to be on time. Thus the penalties for not adhering to the norms and expectations surrounding the ritual communicative events in the pre-match are made clear early in the season, by explicit mention of the forfeiture of match selection through non-conformity to accepted practice. 
Following the team meeting the next event listed on the match day poster is the match kick-off time. However, there are several events that take place in the intervening period that can be regarded as ritual, due to their repeated nature and the important function they have in marking the passage of time and preparing the players.

Immediately after the team meeting, the players get changed into their kit for warm-up and go outside to run through warm-up drills, led by Parky. The warm-up lasts about twenty minutes and the players then return to the team room to get changed into their match jerseys.

Once all the players are ready, and with only a few minutes until kick-off, the players form a series of huddles. Firstly they split into huddles based on the positional CofPs with the Forwards sometimes further splitting into Loosies and Tighties (although as noted in Chapter 4, this further split was abandoned around halfway through the season). Each huddle is led by its respective member of the team captaincy: the Tighties by Jon, the Loosies by Mozza and the Backs by Bug. Jon then leads a team huddle immediately before the players leave to go out onto the pitch and start the match. Both of these front-stage events (within the team) may be regarded as rituals because they always take place at the same time and construct the space around them in the same manner. This has the effect of framing each as a particular communicative event with well-defined norms and expectations that draw in part from rugby ideology and in part from locally negotiated practice. Thus when they are enacted the very performance of them indexes team identity. As it is the leaders who are the main performers in these rituals they may also be regarded as instances of leadership practice. While it is the repeated performance, not the content that makes these events rituals, the content is highly formulaic and repetitive and this is the focus of the next section.

\subsection{Routine and formulae}

The ritual nature of pre-match communicative events indirectly indexes rugby culture because they form part of a shared global repertoire of rugby ritual (Goodger 1986; MacLean 2004; Sheard and Dunning 1973). However, they are ritual not because they contribute to this indexicality but because they do so in a repetitive manner. In other words, it is the repetition of the same structure that makes these communicative events ritualistic rather than simply indexical, showing that in this case ritual may be regarded as repeated indexicality over time. Similarly, formulaic language only becomes 
formulaic through repeated use (Kuiper 2009). Thus the routine structure of the team meeting and the pre-match huddle, and the formulaic language within them, are a result of the negotiated (i.e. repeated) practice of how these events are performed in the Prems. In other words, as the way of enacting the huddle or team meeting is determined through negotiation, and the demonstration of successful negotiation requires the communicative event to take place more than once, it is repetition over time that determines a routine practice for performing these rituals, further enhancing their ritualistic nature.

Formulaic language can therefore be regarded as a part of the repertoire of the CofPs in the Prems. It is used in training sessions and matches in the form of compliments and comments, thus building solidarity among the players (Kuiper 1991). This is shown most saliently in the repeated formulaic compliments used by the Forwards to each other in lineout practice (Chapter 4) such as nice throw or shot (player name). However, formulaic language is also part of leadership discourse in the Prems, through the use of formulaic phrases in the routine structure of the ritual events that take place pre-match. This section will first examine the routine structure used in the ritual events described above before examining the formulaic language that is used within this structure.

\subsubsection{The team meeting}

When it is time for the team meeting to begin Tommo and Parky move to the centre of the team room, having checked that the players are all present. As the time has been set in advance, this is usually all that is needed to signify that the team meeting is about to begin. Tommo will typically use a pragmatic particle such as okay or right-o to signify the start of the meeting, as shown in Example 7.1. The function of these pragmatic particles is to position Tommo as the speaker and the players as hearers, and to make sure that they know that he has the floor (c.f. Chan 2007; Holmes and Marra 2004a). This may be seen to be a negotiated part of the teams' shared linguistic repertoire; the players know that a pragmatic particle, uttered by Tommo, at this point in time on a match day means that the team meeting is about to start, and that they should pay attention. 


\section{N. A. Wilson}

Example 7.1: Team meeting opener

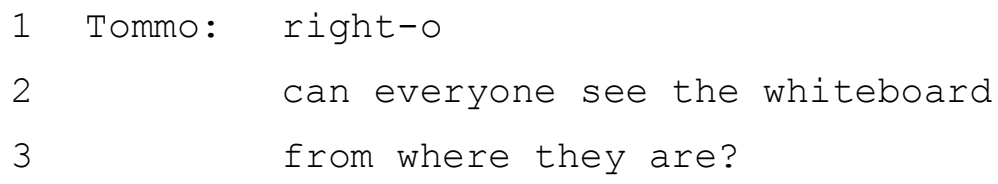

Not every seat in the team room commands a good view of the whiteboard. However, the question in Example 7.1 functions as a directive to the players to pay attention and makes the whiteboard and the coaches the focus of attention. A similar interaction takes place at the start of every team meeting. However this is not the only formulaic phrase used to structure the meeting. In fact the whole meeting follows roughly the same pattern every week. While this could be attributed to the practice of chairing meetings that Tommo may have acquired in other (workplace) CofPs, the adherence to a routine structure also marks out the team meeting as a specific communicative event that forms part of the ritual interaction of the pre-match build-up. By following the routine topic structure, the players recognise that this is an event in which the coaches hold the floor for the duration and in which the players should listen and remain silent. Moreover, due to its ritual position in marking the beginning of the match day it also signals to them that by the end they should be ready to go outside to warm-up. Thus the routine structure combined with the ritualised spatial and temporal context of the event identify it as a team meeting and indexes the norms of interaction that have been negotiated as appropriate for it.

The ritual components of the structure of the team meeting are identified below. The analysis is based on a comparison of all recorded team meetings and the process of establishing the commonalities between them. Thus the topics and speech acts that are listed below may be regarded as the minimum components necessary to perform the team meeting, while on some occasions extra items may be added, such as drawing on examples from professional teams (e.g. Super Rugby teams) to support the important areas of play. Furthermore, the order listed here is not absolute, and some of the topics may recur throughout the meeting 22 .

\footnotetext{
22 Items marked with * are however, temporally fixed in that their structural importance in ritualising the event is such that they can only function in a particular position in the temporal structure of the event.
} 
1. *Meeting opener - discourse marker such as right (or defines the event by saying team meeting)*

2. What has been done in training that week

3. Relevance of training to the match

4. Important areas of play e.g. rucks, what needs to be done to achieve these.

5. Reference to previous matches and place in the season

6. Acknowledge the whole squad is there to play, not just the starting line-up

7. *Give Parky opportunity to contribute*

8. *Parky endorses what Tommo has said (may add detail to key areas of play)*

9. *Tommo specifies times of warm-up and kick-off - this functions as closing the meeting*

Clearly, the team meeting is routine not only in the topics it covers, but in the limited floor-holding sequence. In other words, the only speakers are Tommo and Parky. Tommo is always the initial speaker with Parky's turn arising at the end of Tommo's main contribution. Tommo then closes the meeting, not Parky. This positions both Tommo and Parky as separate from the players, not just as leaders but as the most core members of the Leadership CofP. By both contributing in a supportive, organised manner they perform a distributed form of leadership while Tommo's opening and closing of the meeting indexes his hierarchical position and constructs him as the lead in this event (c.f. fitness training in Chapter 5 where Parky led). The team meeting is the ritual event that constructs their authority in the team most saliently in the pre-match. In contrast, the pre-match huddle, which takes place when the players return from warming up is a ritual event that is purely for the players, so much so that the coaches do not join it, and often leave the room. As mentioned previously, the pre-match huddle was the only huddle I was never invited to join, and I would not have felt comfortable in doing so as a non-player.

\subsubsection{The pre-match huddle}

The pre-match huddle contains no new information for the players that has not already been introduced in the team meeting, it serves as a ritual encounter that will motivate 


\section{N. A. Wilson}

the team and enhance solidarity (Goodger 1986). This is achieved through reference to territoriality (as discussed in Chapter 6), a high frequency of swearing and an emphasis on inclusivity. That these features are consistently used in pre-match huddles in this team shows how performances such as the pre-match huddle, although symbolic of rugby culture, are locally negotiated in the manner of their performance. The pre-match huddle is not routine in structure to the degree that the team meeting is, however. It features opening and closing acts, the former being the exhortation to huddle up or get tight, and the latter being the brothers call, which has been referred to in previous chapters and will be examined more closely in the next section. The pre-match huddle is routine in the way that it takes some of the content of the team meeting and re-packages it in a more emotive style, thus itself being an act of fertile mimesis, where the same content is used but to enact a different ritual. This is achieved through speech volume, repetition, swearing and tag questions. The repetition of topics from the team meeting means that a part of the ritual for the pre-match huddle is the repetition of content, which further provides an indexical link between the leadership discourse of Tommo and Jon. It is thus the relationship between the team meeting and the pre-match huddle that shows how the routines used in enacting ritual front-stage events such as these are part of both leadership practice and team identity construction.

The following two examples highlight the differences and similarities between the team meeting and pre-match huddle. Both are taken from a single match in order to show how the same content, formulated by Tommo, is restyled by Jon to fit the formula of the huddle. Jon restates points in the pre-match huddle that were made by Tommo in the team meeting, but in a style particular to the pre-match huddle and characterised by his use of swearing and the pragmatic particles eh? (line 10) and alright? (lines 9 \& 11-14). The first extract from is from the middle section of the team meeting in which Tommo details the objectives for the team's performance, and both extracts have been colour coded to show where they are thematically linked. 
Temporality: The renegotiation of leadership and identity

\section{Example 7.2: Team Meeting}

\begin{tabular}{|c|c|}
\hline Tommo: & it might not happen in the first scrum \\
\hline 2 & it might not happen in the- \\
\hline 3 & in the scrum in the sixtieth minute \\
\hline 4 & but by the end of the game \\
\hline 5 & I want to make sure \\
\hline 6 & and I want see from you guys \\
\hline 7 & that we dominate their tight five \\
\hline 8 & I want us to be aggressive entering the \\
\hline 9 & collision zone \\
\hline 10 & so when we're going int- \\
\hline 11 & into breakdowns when we're carrying or \\
\hline 12 & going into a tackle \\
\hline 13 & I want to see us aggressive up front \\
\hline 14 & and I want to see that all day \\
\hline 15 & I want it controlled \\
\hline 16 & but I want it aggressive \\
\hline 17 & out of the aggression it means \\
\hline 18 & that we go forward \\
\hline 19 & exactly what we talked about Tuesday night \\
\hline 20 & what we lacked against [Opposition Name] \\
\hline 21 & last week \\
\hline 22 & it's us going forward all day \\
\hline 23 & and it's got to happen up front okay? \\
\hline 24 & {$[\ldots]$} \\
\hline 25 & the backs .. \\
\hline 26 & organisation .. on both attack and defence \\
\hline 27 & so that's from set piece.. \\
\hline 28 & Willie be organised \\
\hline 29 & know what we're going to do \\
\hline 30 & before we even get there... \\
\hline 31 & if we're going to have a go at them wide \\
\hline 32 & let the forwards- let the forwards know .. \\
\hline 33 & chips or or pies ${ }^{23}$.. so that they know \\
\hline
\end{tabular}

${ }^{23}$ chips and pies are both codes (not the real ones, although semantically similar) that the forwards use to determine which players will be first to the tackle situation (the breakdown). The forwards arrange 


\section{N. A. Wilson}

$\begin{array}{ll}34 & \text { exactly the pods are going where and who's } \\ 35 & \text { going into what position }+ \text { being organised } \\ 36 & \text { lots of communication eh? } \\ 37 & {[\ldots]} \\ 38 & \text { we're playing the right end of the field } \\ 39 & \text { we dominate the viel- the field possession } \\ 40 & \text { points'll roll on } \\ 41 & \text { tha- they're the results .. } \\ 42 & \text { and that's something } \\ 43 & \text { that I want us to do today } \\ 44 & \text { but I want us to do it a step above } \\ 45 & \text { that we've done it in the last five or six } \\ 46 & \text { weeks ... } \\ 47 & \text { okay these guys are not coming out here } \\ 48 & \text { to lie down for us } \\ 49 & \text { regardless of the points } \\ 50 & \text { or where they are on the table } \\ 51 & \text { and where we are }+\end{array}$

\section{Example 7.3: Pre-match huddle}

\begin{tabular}{|c|c|}
\hline Jon: & forwards \\
\hline 2 & we're in these cunts' faces all fucking day \\
\hline 3 & we dominate these cunts \\
\hline 4 & we get fucking good ball for Willie \\
\hline 5 & so Tui and the backs and Bug can do \\
\hline 6 & whatever they fucking want \\
\hline 7 & you know \\
\hline 8 & do the magic alright? \\
\hline 9 & I want to see fucking wingers scoring in \\
\hline 10 & the in the corners alright? \\
\hline 11 & us fucking walking back going fucking \\
\hline 12 & let's start again let's fucking start again \\
\hline 13 & {$[\ldots]$} \\
\hline
\end{tabular}

themselves in pods of three players, one closer to the inside of the pitch and the other to the outside, and each pod is labelled either chips or pies. The backs can thus tell the forwards which direction they are going to take the ball. 


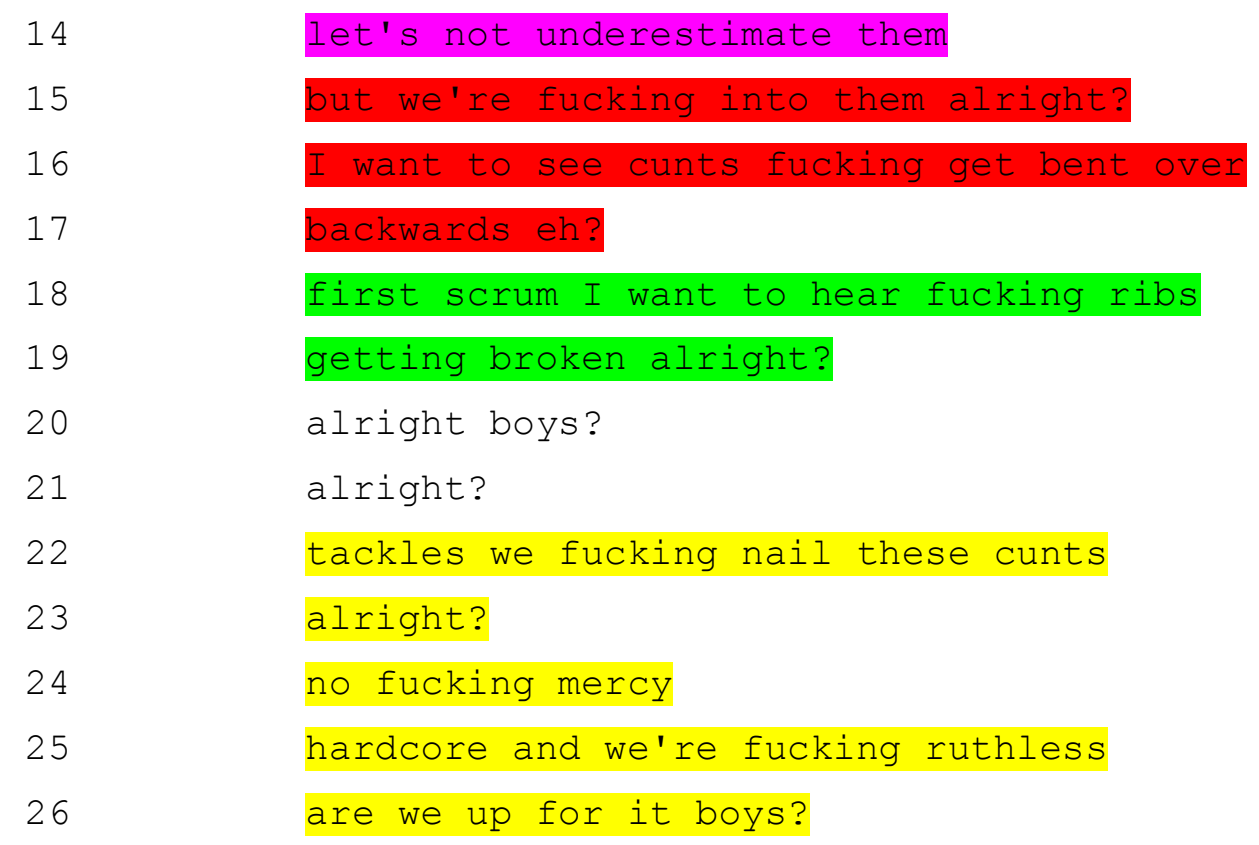

As can be seen from the comparative length of highlighted sections that are linked through topic, Jon is rather more succinct in his speech to the players than Tommo. Looking more closely, the similarities and differences between the two examples can be seen clearly in the two statements:

Tommo: I want us to be aggressive entering the collision zone (Example 7.2, lines 8 \&9)

Jon: I want to see cunts fucking get bent over backwards eh?

(Example 7.3, lines 16 \& 17)

Both statements start, I want, making a clear demand of the players. However, while Tommo's demand conveys an official tone by using the rugby terminology of collision zone and is somewhat oblique by referring to being aggressive, Jon's is exaggeratedly explicit in the imagery of the opposition being bent over backwards. Additionally, the swearing, as described above, functions as a solidarity marker as well as an indirect index of masculinity.

The difference in the discursive styles is also reflected in the way in which the space of the team room is used during the two events (MacLean 2004; Wilson forthcoming). 
During the team meetings the players are all seated while the coaches stand, positioning the players as an audience rather than participants (Howells and Becker 1962; Sommer 1961). During the pre-match huddles on the other hand, the players stand, shoulder to shoulder, with arms around one-another's backs. This gives each equal participatory status while the boundary created by the players' bodies signifies that they are a group separate from outside control. Furthermore, the spatial organisation of the huddle zones off an area of the team room as the players' territory, and positions the inside of the huddle as the front-stage in which they can construct their own version of their identity (Mondada 2009). Thus the spatial organisation of each event is as much a part of the ritual practice that specifies it as a discrete communicative event as the discourse that takes place within this space. Furthermore, it is the interplay between discourse and space that marks the huddle out as a ritualised event; the huddle does not begin until every player has huddled up (Goffman 1967).

While this section has so far looked at the formulaic structure of the pre-match communicative events, the formulaic nature of the language used within these events is another way in which language is temporally situated in the discourse of the Prems. This represents the most micro of the levels of repetition mentioned in the introduction to this chapter and is the focus of the rest of this section.

\subsubsection{Formulaic language}

The formulaic phrases that the leaders draw upon in their ritual performance not only underline these events as ritual, but also serve as an indirect indexicality of rugby ideology by indexing a power difference between leader and player, an intention to beat the opposition, or solidarity among the team. The three formulae that are found in the pre-match rituals are identified below, and are used by both coaches and player leaders. They are analysed as formulae because they can be considered as phrasal units that recur in some form in every pre-match communicative event. This is not to say that other formulae do not exist within these rituals; rather these occurred in every recorded example whereas others occurred more sporadically. Examples of each are drawn from Example 7.2 and Example 7.3 above in order to show them in context. 
Temporality: The renegotiation of leadership and identity

\section{Formulae of power}

a. I want you/us to [action] ([temporal/spatial locator] $)^{24}$

b. I want to see (us) [action]

c. I want it [adjective]

e.g. I want you to fucking smoke that number eight (Example 6.3, lines 41 \& 42)

I want to see us aggressive up front (Example 7.2, line 13)

I want it controlled (Example 7.2, line 15)

I want to see fucking wingers scoring in the in the corners (Example 7.3, lines 9 \& 10)

These three formulaic directives all emphasise the difference in power between the leader and the players (c.f. Kuiper and Lodge 2004; Vine 2004). While the majority of the realisations of formula $a$ are mitigated somewhat by the use of we rather than you, the use of I want is a clear indication of the power relations between the leaders and players in the pre-match ritual; the expectation is that the leaders talk and the players listen. The information conveyed in these formulaic utterances is hardly unexpected as they are all centred on a desire for actions that will ultimately lead to winning the match. Therefore, making such explicit demands is not likely to make players any more likely to do these things. Highlighting the power difference indexes a rugby ideology of an authoritarian coach. This may help to focus the players on the match by making the directives personal rather than for the benefit of all, as is more often the case in the Prems. In repeating the formula used by Tommo in the team meeting, Jon directly indexes the authoritative stance of the head coach in his directives. The formulaic construction of directives may thus be considered a part of the repertoire of the Leadership CofP and a ritualised practice for enacting the team meeting and pre-match huddle. As with many other discourse features analysed in this thesis, the use of formulaic language functions on multiple levels of discourse, "doing” leadership, constructing identity and enacting ritual, all at the same time.

${ }^{24}$ ( ) indicates an optional part of the formula while [ ] indicates a component that may take various forms but must convey the specified semantic content. Text without either are necessary words, while / indicates a choice. 


\section{N. A. Wilson}

\section{Formula of intent}

[team/subset of team] ([verb indicating intent]) dominate [opposition/gameplay]

([spatial/temporal locator])

e.g. we dominate their tight five (Example 7.2, line 7)

we dominate these cunts from the first whistle (Example 7.3, lines 3 \& 4)

The verb to dominate seems to be the favoured way of expressing how the team should perform. It is most frequently used with we (the team, including the leader) as the subject and either the opposition or the game as the object of domination. It may be further specified that the domination occur in a particular location such as on the pitch or at a particular time such as in the first half, or from the first whistle (i.e. the start of the match). What seems to be invariant is the use of dominate as the verb. This is interesting as the use of such a powerful verb with its connotations of hegemonic masculinity (Schacht 1996) creates a stance that suggests aggressiveness and power as core values. While this is not an identity useful in training (or indeed outside the rugby club), when players are about to run onto the rugby pitch and take part in the first physical exchange of the game, language such as this can motivate them. This is after all the main function of the pre-match ritual, to prepare players to go into what amounts to a combative sport.

\section{Formula of Solidarity}

([Attention-getter]) brothers on three (hands up high) one two three brothers

This is the formula that closed every huddle except the huddles on the final day of the season as discussed in the next section. This formula for closing huddles is similar to that which is used by many rugby teams ${ }^{25}$ except that, in my experience, it is the team name or nickname that is shouted rather than brothers. This team-specific formula for signalling the end of a huddle is an example of the way global rugby practices can be renegotiated at a local level in order to create a unique identity for the team. In the case of the Prems, this formula was originated by Jon, who suggested it at the start of the

\footnotetext{
${ }^{25}$ It is true for all the club teams that I have played for and against, as well as the Prems, but, in my experience, not for teams with a less coherent identity such as representative sides (in Scotland) where players only meet a few times in a season.
} 
season as a means of marking the Prems out from the other teams in the club who all used the traditional formula. By using the kinship term brothers this not only engender solidarity among the players but may also be seen as indexing a Māori identity (Bell 2000; Johnston and Robertson 1993; Stubbe and Holmes 2000). As mentioned in the previous chapter, several aspects of the team's discourse can be analysed in this way.

Consequently, as discussed in the next section of this chapter, the final match of the season, without Jon's leadership, proved to be one of the most interesting matches because it involved consideration of how the players went about trying to fill the gap left by Jon. They were forced to renegotiate not just the roles of the leaders within the team but also the way in which their identity was constructed pre-match. On this occasion, Mozza led the huddle and initiated the closing. Rather than shouting brothers, they shouted the name of the club. I interpret this as a clear indication that the identity of the Prems was markedly changed by the departure of Jon. The brothers call was a practice that was introduced by Jon, and enhanced the solidarity of the team. Mozza either did not see it as appropriate for him to use it, or this was an attempt to renegotiate the practice of the team under his leadership, indexing the commitment to the club rather than to one another.

It is this final match that forms the focus of the next section and the way in which Jon's departure allowed for a renegotiation of leadership practice and the pre-match ritual.

\subsection{Re-negotiating the routines of leadership ritual}

Ritual, routine and formulaic language depend on repetition of events over time (Alexander 2004; Kuiper 2009; Smith 1980), and consequently they can only be analysed as either ritualised or formulaic when viewed in the context of the history of a community. In the moment they are simply "snapshots" of a CofPs negotiated practice for performing specific communicative events (Jaffe 2009). However, as shown in the previous chapter, identity can only be analysed with respect to an accrual of stance (and hence practice) over time. Thus looking at what might constitute formulaic discourse in ritual events entails examining the most frequent structures and phraseology. However, by this it is not meant that formulae exist only in hindsight and that what have been retrospectively identified as ritual events do not have symbolic meaning at the time they are performed. Quite the opposite, for the participants, each performance of a 
ritual event is symbolic because it has a place in rugby and club culture, with similar events taking place in all rugby teams (MacLean 2004; Schacht 1996; Sheard and Dunning 1973). The formulaic language used in performing the ritual events is also a part of the repertoire of the team and the CofPs within. While these rituals may begin, at the opening of the season, as negotiations of practice, with each repetition this is renegotiated. If there is little change in a particular communicative practice over time, it can be analysed as routine, although it is not off the table for negotiation. As this section demonstrates, formulae can be changed as part of a renegotiation of practice, for formulaic language is a part of practice. In the Prems it is part of leadership practice, because by performing the rituals of the pre-match the coaches and captains enact their identity as leaders on the front-stage of team discourse.

In the build up to the final match of the season, a situation arose which meant that the leadership of the team and thus the practice of player leadership was up for renegotiation. Jon left the club to pursue a professional rugby career in Australia and thus the next in line for the captaincy was Mozza. Bug was injured at this time and so although he negotiated an identity somewhere between player and coach (see Chapter 6), he was ineligible to be captain for this final match. However, as mentioned in Chapter 5, Mozza did not perform leadership in the same way as the core members of the Leadership CofP. When interviewed early in the season about leadership and his role as co-captain, he told me that he did not really like addressing groups of people, thus suggesting that performing the pre-match huddle as Jon had done would be a challenging feat for him. However, when this duty did fall to Mozza, in the final match, what transpired was a renegotiation of the way in which this ritual was enacted. While following the formulaic constructions used by Jon throughout the season (c.f. Example 7.3), the pre-match huddle became a joint, rather than solo, performance as illustrated in Example 7.4.

\section{Example 7.4: Final pre-match huddle}

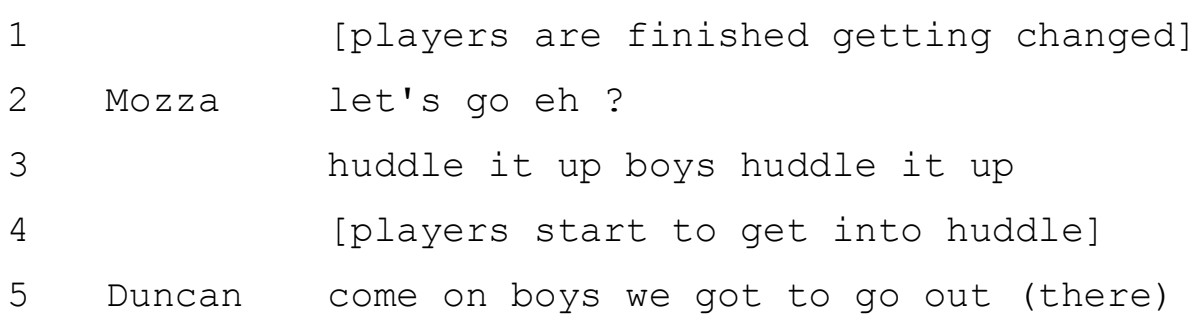


Temporality: The renegotiation of leadership and identity

\begin{tabular}{|c|c|c|}
\hline 6 & Mozza & yep \\
\hline 7 & Giles & let's go $=(\quad)=$ \\
\hline 8 & Mason & $=$ let's go = get in a huddle eh \\
\hline 9 & & listen up to Mozza \\
\hline 0 & Mozza & let's go huddle up quick \\
\hline 1 & Giles & we know how to play .. let's go \\
\hline 2 & Mozza & let's go we've got one minute \\
\hline 3 & Unknown & WOO \\
\hline 4 & Mason & fuck we run this show boys \\
\hline 5 & & .. eighty minutes of your life that's all \\
\hline 6 & Mozza & listen right eh ? \\
\hline 17 & & last fucking game of the season boys .. \\
\hline 8 & & we don't leave anything in here eh ? \\
\hline 9 & & we don't leave anything in here \\
\hline 0 & & we go out there \\
\hline 21 & & we fucking dominate these cunts \\
\hline 22 & & from the first whistle \\
\hline 23 & & let's fucking take these cunts to pieces \\
\hline 24 & & eh ? \\
\hline 25 & & it's us all day eh ? \\
\hline 26 & & [team name] boys \\
\hline 27 & & [team name] we fucking (pull our) boys \\
\hline 28 & & $=$ you know what we gotta $=$ do eh ? \\
\hline 9 & Bug & $=$ our final eh $?=$ \\
\hline 30 & Mozza & physical and smart \\
\hline 1 & & let's play physical and let's play smart \\
\hline 32 & & $=1$ guys are we doing this eh ? = \\
\hline 33 & Mason & $=1$ (don't) fucking lose this boys $=$ \\
\hline 34 & & fucking up our $=2()=$ \\
\hline 35 & Bug & $=2$ our = final boys eh ? \\
\hline 36 & & our fucking final \\
\hline 37 & Unknown & hey let's do it let's get out there \\
\hline 38 & Mozza & let's go let's fucking get in to it boys \\
\hline 9 & & .. [Team name] on three \\
\hline 40 & Mason & nothing left on that field boys \\
\hline 1 & & nothing on that field \\
\hline & Mozza & [team name] on three \\
\hline
\end{tabular}




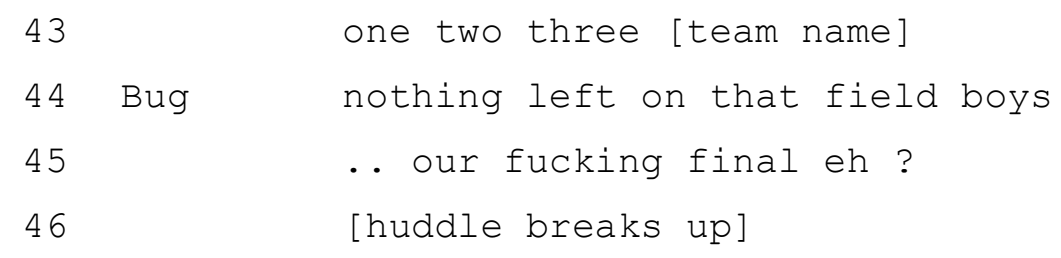

In this pre-match huddle, unlike in any others, there are multiple speakers. However there are formulaic phrases that signal that this is a pre-match huddle such as we fucking dominate these cunts (line 21). Furthermore, it conforms to the spatial and temporal requirements of a pre-match huddle by taking place in the team room, with the players in a tight circle, immediately before kickoff. Mozza uses a structural element favoured by Jon: huddle it up (line 3) to signify the start of the huddle thus enacting the ritual of the pre-match huddle according to the established structural formula. Yet the players do not respond as promptly as they did for Jon and thus it might be postulated that Mozza's initial attempt to index Jon's leadership stance is rejected. At this point Mason, a peripheral member of the Leadership CofP, steps in to aid Mozza in performing the huddle, by reiterating his call to form a huddle (line 8). One could analyse Mason's intervention as a move to establish himself as an alternative candidate for the team captaincy. However, my view, based on the co-operative norms of the Forwards and Leadership CofPs, is that while Mason does align himself more closely with the core of the Leadership CofP by issuing a directive in this situation, his actions are focused on performing the huddle successfully; when Mozza appears to struggle with this, he follows the established formula. Moreover, Mozza's reaction (line 16) indicates that he expects the players to behave as if he were Jon and not to talk. In fact on the recording his loud, staccato intonation suggests that he is annoyed with the lack of attention he receives. His demand for attention does however win him the floor for some time, during which he indexes Jon's pre-match style through his use of swearing and repetition, as well as using formulaic phrases such as we dominate these cunts (line 21), and violent imagery such as let's fucking take these cunts to pieces (line 23). He also indexes their territorial identity through his use of the club name (lines 26 \& 27), a feature which is repeated at the end, in place of the brothers call.

In performing each of these ritual leadership practices, established by Jon as way of performing as captain in a pre-match huddle, Mozza appears to have been successful in 
leading the huddle, albeit with a slow start. However, just as his extended turn appears to be ending, Bug's and Mason's comments (lines 29-36) overlap not only Mozza but each other, creating a sense of the three players jockeying for position as speaker. This would not be unusual in a training session huddle or even a half-time huddle, but the way in which overlapping speech occurs here is not the norm in the pre-match huddle and may suggest that Mozza's stance as sole leader is rejected, at least by the other players who see themselves as potential leaders. Alternatively they may simply have interpreted Mozza's use of the team name as a closing move and tried to add their support by contributing formulaic phrases such as our (fucking) final (lines 29, 35, 36 \& 45) and nothing (left) on that field (lines 40, 41 and 44). That the majority of Mozza and Bug's contributions to this huddle (apart from Mason's initial intervention) consist of these formulaic phrases which appear frequently in sports media discourse (File forthcoming), may indicate that they are performing solidarity work in support of the more detailed motivational discourse supplied by Mozza. We could therefore interpret this as a renegotiation of the pre-match huddle routine and of player leadership practice that is modelled on a co-operative approach as espoused by the coaches. In other words, this huddle marks a renegotiation of practice, using formulaic language and routine structure to incorporate existing practice into new.

Had this not been the final match of the season, it would have been interesting to see whether Mozza built on the renegotiation of the leadership structure and the contextual norms of the pre-match huddle by adopting a different leadership stance in subsequent matches and if a new leadership practice emerged from this. However, when I did return to the team the following season to conduct feedback sessions with the players, Mozza was still a player, but no longer part of the captaincy structure. In fact the captaincy had been refused by both Mozza and Mason as they did not feel they could fill Jon's shoes. The new captain was Ata, a player who did not appear at first to be an obvious leader, but who was recorded in numerous back-stage conversations talking to the coaches about his performances and asking for advice on how to improve, not just in terms of his rugby performance but how to think about his role in the team.

Interestingly, he was also one of the players who showed most interest in this research, regularly asking, when we spoke, what I had found so far. Thus Ata may have been performing back-stage leadership behaviour but not front-stage, suggesting that the 


\section{N. A. Wilson}

front-stage performances of leadership discourse are predicated on the work done in the back-stage (Richards 2006).

\subsection{The last rites of the team CofP}

The loss of Jon as an influential leader signalled a major change in the way the team constructed its identity and was perhaps the beginning of the end for the team as a CofP. Example 7.4 shows that moves were made towards renegotiating player leadership practice. Had this occurred midway through rather than at the end of the season this renegotiation might have been completed. This could have led to a resultant change in the practice of pre-match ritual and the evolution of a somewhat different team CofP (Moore 2010a). The "official" end of the Prems however, was after the end of the final match and was marked using the ritual of the post-match huddle.

\subsubsection{The post-match huddle}

Like the team meeting, this event is coach-led, but is a form of huddle as defined by the spatial organisation of the team. It always takes place on the pitch, immediately after the final whistle and functions as an evaluation of the match. Like the team meeting, it follows a formulaic structure, which is dependent on whether the match has been won or lost. Sadly the Prems did not win enough matches for an analysis to be made of a formulaic winning post-match huddle, and consequently the following is the routine structure when the match has been lost:

1. *Directive to join the huddle*

2. *Sympathy shown for players e.g. I know you're disappointed*

3. Point out the positives from the performance

4. Reference the past matches in comparison

5. Specify what will happen in the coming week

6. Look ahead to the next and future matches

7. *Final compliment on performance as closing remark*

While the order of these topics is fixed to a certain extent (in that they are introduced in this order) they may be interwoven throughout the huddle, as is seen in Example 7.5. Post-match huddles can be likened to post-match media interviews by their temporally defined thematic structure. Media interviews address the same topics, but they do so in a more rigid order (Emmison 1987, 1988; File forthcoming). 


\subsubsection{The final post-match huddle}

What is interesting in the final post-match huddle is that it adheres to the same structure as other (losing) post-match huddles, by using the temporal references of immediate past (the match), the more distant past (prior matches), the immediate future (training) and the more distant future (upcoming matches), but here they are used to review the season as a whole. In this regard this particular post-match huddle performs a dual ritualistic function. Not only does it mark the end of the match, but it also marks the end of the season.

Tommo and Parky signal the end of the team as a CofP by talking about what various players will be doing during the off-season as well as thanking the players for their commitment over the course of the season. In this huddle, Tommo and Parky both acknowledge that the players will continue to be members of the club and that an enduring membership exists. However, the team that has been in existence for this season as the Prems and which has forged an identity through their shared interactions has now come to an end. This final post-match huddle is shown in its entirety in Example 7.5. It neatly links together the same four aspects of time that are used in other pre-match huddles, but instead of using the current week as the focus, it reviews at the season as a whole referencing the immediate past (the match), the more distant past (the season), and the immediate (off-season) and distant (next season) future. In order to show how these four times are interwoven, each time has been highlighted with a different colour. The current match is yellow, the season is grey, the off-season is green and the next season is red.

\section{Example 7.5: Final post-match huddle}

$\begin{array}{lll}1 & \text { Tommo: } & \text { come in boys come on in together } \\ & \text { [players huddle round] } \\ 3 & \text { Tommo: } & \text { okay um . } \\ 4 & \text { obviously not what we- } \\ 5 & \text { what we were looking at to finish off } \\ 6 & \text { but that's the way the ball bounces } \\ 7 & \text { and there's got to be a winner and a loser } \\ 8 & \text { and it er obviously wasn't our day } \\ 9 & \text { but um on the season thanks very much } \\ 10 & \text { there's been some bloody good efforts eh ? } \\ 11 & \text { a lot of you guys have got some rep rugby to }\end{array}$




\section{N. A. Wilson}

kick on with after this

which is outstanding whether it-

whether it's B's colts.. nineteens .. the

Maoris whoever it is go well

enjoy yourselves keep ( ) (working fulltime)

to the colts that came down today

guys .. who made debuts...

awesome thanks very much eh

you guys really shone out

fantastic stuff

All: [applause ]

Tommo: we- we've done um

we've done a lot of good shit this year

secured ourselves in the Coronation cup

er Milton Shield for next year..

and that's always a big- a big ask

now you look at Avalon

they can't say the same thing...

they're gone and we're safe for next year

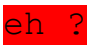

we've had some pretty good er

pretty good hit outs

this Coronation cup round

and Coronation Cup rugby's good rugby eh

a step on from all the other shit

you go through year in week in week out

so it's-it's a taste now for next year

so er for those guys that have got rep stuff

to go on with

you know enjoy yourselves ..

do yourselves proud do your club proud

a small break for those that aren't

.. playing rep rugby

then we'll be back into it

for our summer programme together

that's something to be excited about

.. get keen on ..

but um hey keep your heads up boys

you've all done yourselves proud

you've done the club proud

me and Parky are proud as of you 
Temporality: The renegotiation of leadership and identity

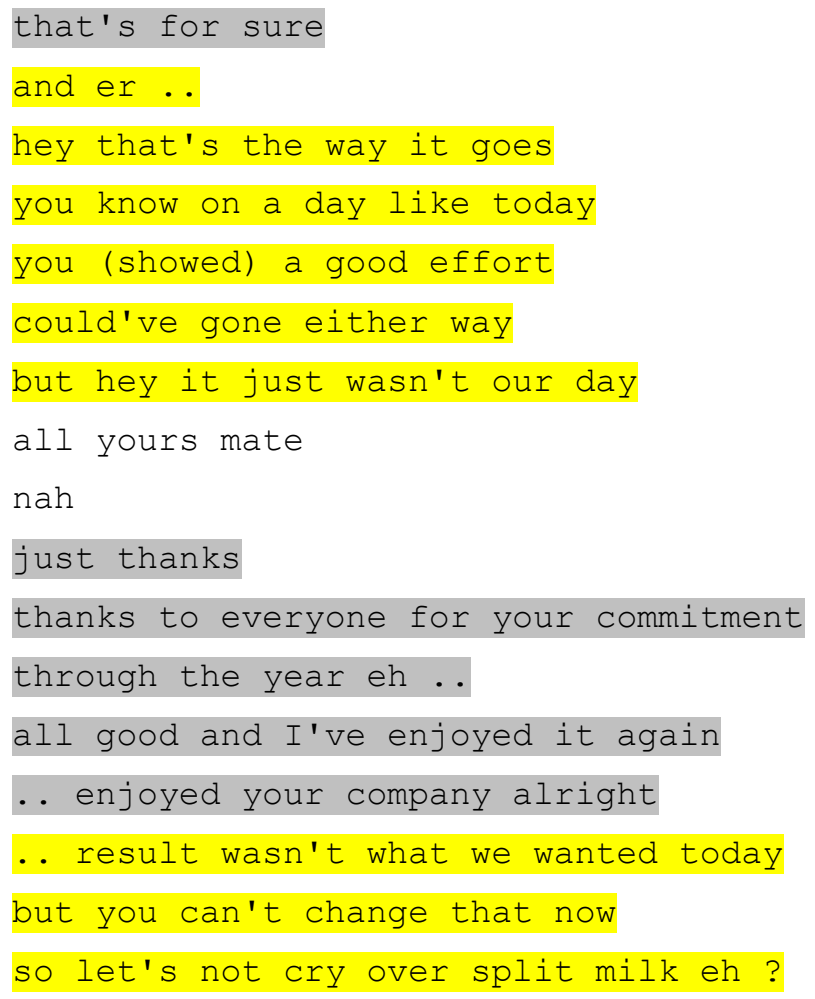




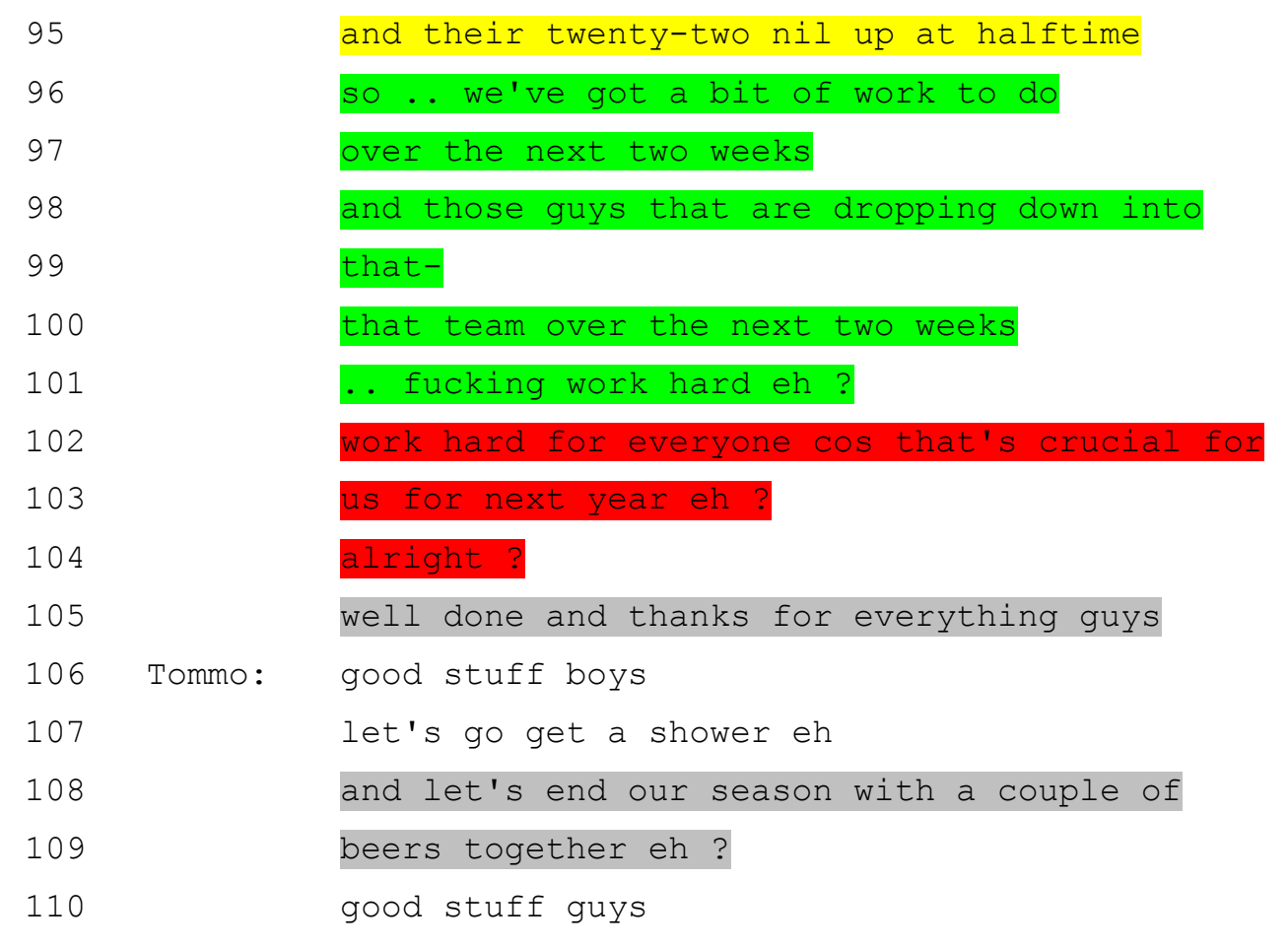

As can be seen from the colour coding of the different points in time that are referred to in this example, the official end of the season is marked by looking back and looking forward; the end of the Prems for this year is the beginning of the potential Prems for the next year. However, in addition to looking back at the immediate and distant past and looking forward to the immediate and distant future, this huddle also has to function as a post-match huddle; to comment on the result and team performance in the match that has just been played. Just as in other post-match huddles, Parky and Tommo do not dwell on the defeat, but accentuate the positive aspects of the team's on-field performance. In doing so they are following the formulaic construction of the postmatch huddle, drawing on the established practice to construct a team identity that is resilient in defeat and indexing the leadership stances that they have taken in other, similar, situations. Likewise, in other post-match huddles they talk about looking forward to the next match. As there is no next match in this instance (for the Prems as a team) the off-season activities take the place of the immediate future in this post-match huddle. Therefore, while this huddle is unique in that it provides a marker for the end of the season and so the content is somewhat different to the usual post-match huddle, it nonetheless follows the routine for this type of huddle, making it a ritual event and thus enhancing the illocutionary force of the statements made within it (Levinson 1983). In other words, as this communicative event is a post-match huddle, it already carries with 
it the performative aspect of signalling the end of the match day. Thus the addition of signalling the end of the season is more powerful here than it would be as a speech event in its own right.

This was referred to earlier as the "official" end of the Prems as a CofP. However, it would be more accurate to say that this was the coaches' performance of the end of the Prems. The players carried out their own ritual end of season performance on the bus home. Unfortunately it was not possible to record this so my ethnographic notes will have to suffice:

Research journal extract - 29/07/2009

On the bus back we stopped at a bottle shop and Tommo and Parky bought several crates of beer for the boys. These were distributed amongst the players. We stopped at the top of [Place Name] and each of the newer players were held upsidedown by their ankles and made to chug a can of beer, in the full glare of the bus headlights. Had we not run out of time because the bus was due back I probably would also have had to do it. Mason prodded me in the chest on the way back onto the bus and said that he hadn't forgotten about me.

At the time I did not reflect much on the significance of this event, as I had become a peripheral member of the Prems. Furthermore, as an ex-rugby player myself this sort of thing was not a new experience. In fact in many regards I thought it quite tame compared to some of the rugby initiation rituals I have heard of, witnessed or taken part in. What I found unusual however was that this initiation of the new players into the team happened at the end of the season, not on their first appearance for the team. Perhaps the significance of this is that they are not just new players, but by still being present at the end of the season they have demonstrated that they have not only played for the Prems as a rugby team, but also that they have moved from peripheral to core members of the team CofP. Whatever the reason, despite being ratified by the coaches (they bought the beer), this ritual belonged to the players. It not only formalised the acceptance of players who had not played for the Prems in prior seasons, but like the post-match huddle described above, established a continuity between seasons and paved the way for a new Prems CofP to emerge the next season. 


\section{N. A. Wilson}

\subsection{Temporality}

In this chapter the temporal nature of communicative practice has been the focus of analysis. Formulaic language and ritualised communicative events have been examined as examples of the way in which temporality is manifest in the Prem's discourse. By analysing the formulae present in the structure of communicative events and their constituent language, my intention is to highlight that what constitutes leadership discourse in the Prems is constructed from what has gone before and will have bearing on what comes after. In other words any instance of leadership practice is temporally bounded; it indexes past stances of leadership and will be indexed by future leadership stances. As noted in Chapter 6, identity may be viewed as the accumulation of stances over time (Jaffe 2009; Johnstone 2008). Thus each leadership stance, or instance of leadership practice, feeds into the team identity as a part of team communicative practice.

Therefore temporality should not be seen as simply another aspect of the Prem's discourse. Rather it is the unifying factor that combines leadership, identity and practice. Using examples of ritual and formulaic language it has been shown that ritualised communicative practice can be used to enact leadership and index identities that may motivate the team. Furthermore, each repetition of a communicative event is in effect a renegotiation of practice, even if the outcome of the negotiation is that the practice remains the same. Repetition has been shown to exist on multiple levels, from the repetition of words and phrases at the level of the utterance and within a single communicative event, to the repetition of the process of CofP emergence across rugby seasons. This parallels the way in which indexicality, leadership, and CofP membership have been described as multi-layered in previous chapters. All of these multi-layered constructs are however dependent on the passage of time. They each refer to events or conditions that have taken place at some point in the past. Thus temporality is a vital component in the contextual understanding of discourse (Blommaert 2005). It is also a crucial component for the understanding of the CofP however, as discussed below.

\subsection{Performance, identity, leadership and time}

In the discussions of formulaic language, formulaic discourse structure and the ritualisation of communicative events above, this chapter has looked at leadership as both a performance and a practice. Each performance can be regarded as a move in the 
negotiation of practice. As an event is performed again and again, a practice is established based on the interaction and acceptance of stances made within the event. The integration of formulae and practice may seem an uneasy union, unless one regards formulaic discourse as a part of discursive practice rather than an alternative. The reason for bringing in the performative aspect of discourse and the idea of formulae as an evolving aspect of linguistic practice is to emphasise that in a CofP such as the Prems, which has a clear beginning and end, the passage of time is crucial in the analysis of the identities and practices that it encompasses.

The CofP is not a static construct (Eckert and Wenger 2005; Hughes, Jewson and Unwin 2007; Moore 2010a), yet it is hard to describe a CofP, or indeed a system of CofPs, without describing them at a particular point in time. In doing this there is a danger of overlooking the constant renegotiation and fluidity of practice; in other words the whole point of the CofP model is lost. By saying "this CofP does this", we are effectively turning a dynamic concept into a static description. This is not a weakness in the CofP model however, but in the way in which it is used. In studies that take place over an extended period of time (e.g. Bucholtz 1999; Eckert 2000; Moore 2006, 2010a; Tusting 2005), there is bound to be renegotiation of practice and thus identity, yet often it is a snapshot of practice that is described, albeit a detailed snapshot of the CofP at a particular point in time. In describing the detailed practices of a high school, Eckert (2000) uses the affiliative negotiation of linguistic variables to show that individuals position themselves closer or further from the core of two oppositional CofPs, however she does not describe whether the practices that define the core change over time. Moore (2010a) overcomes this by describing CofPs that evolve from other CofPs as some members of a CofP negotiate their communicative practice in a different (or more extreme way) from others. The evolution of one CofP from another is a concept that has been taken up by this research, and used to examine how leadership practice and identity are negotiated over time through the development of CofPs that form around institutionally defined groupings within a larger CofP. As stipulated at the end of the previous chapter, it is not possible to describe a team identity, only the factors which shape it at different times and how it is constructed in situations over time.

This chapter represents the integration of leadership practice and identity construction within the CofP framework, using the concept of temporality and repetition to link the 


\section{N. A. Wilson}

three. The final chapter summarises the findings of this thesis and how these address the research questions specified in the introduction. 


\section{Chapter 8 Conclusions}

In this thesis, both leadership and team identity have been examined as drawing on spatial and temporal contextual factors in their construction, thus showing that, in this team at least, leadership is a spatialised practice, expressed through the idea that discourse strategies construct the space in which they are enacted as front-stage or back-stage. The analysis that has led to this conclusion has been built upon ethnographically acquired knowledge and extensive study of the literature surrounding the sociology of sport, the discursive enactment of leadership and linguistic construction of identity. These were brought together using the conceptual framework of Communities of Practice, allowing the negotiation of local communicative practice to be related to leadership discourse and identity construction. In doing so, this thesis has treated leadership both as a practice and as an identity, using the concepts of stance and indexicality to link the two. The ethnographic work which underpins this research was, however, primarily facilitated by my own experiences as a rugby player, which has been useful in numerous ways in the course of the research. My knowledge of rugby ideology and the socio-cultural norms of rugby aided in gaining access to a team and allowed me to integrate with it more fully than might have been otherwise possible. It also gave me a background knowledge of rugby culture, expanded through ethnographic research, that allowed me to interpret the interactions of the players and coaches as a "native" of the sport.

In addition to the analyses presented in this thesis, also highlighted is the value of ethnography as a research method in sociolinguistic research. As Chapters 2 and 3 explain, I view ethnography not simply as method for gaining access to a rich source of authentic spoken discourse, but as a crucial tool in developing the analysis of this data. As noted in Chapter 2, this follows the methodological frameworks of Linguistic Ethnography and Interactional Sociolinguistics, both of which depend on a deep, ethnographically acquired, understanding of context in the process of analysing discourse.

The in-depth ethnographic approach taken in this research of observing, interacting with, and recording a rugby team over the course of a rugby season has, in addition to 
providing the socio-cultural knowledge vital for analysis, resulted in the recording of a rich set of recorded interactions. These represent a range of communicative events that, based on ethnographic experience, are representative of the team's discourse: from the ritualised huddles and meetings of the match day, to the back-stage discussion and front-stage explanation of training drills by the coaches. However there still exists some recorded data from this research that has yet to be transcribed, representing a resource for further research, some suggestions of which are contained in the penultimate section of this chapter. Prior to this however, a summary of the conclusions reached in this thesis is provided. This is structured broadly around the research questions specified in Chapter 2, and is followed by a discussion of the implications of this research relating to methodology and theory, as well as a brief discussion of how this research may be relevant to practitioners of sport, in this case rugby coaches and players.

\subsection{Research questions}

The research questions that have been addressed in this research are:

1. How do members of a rugby team construct themselves as leaders through discourse?

2. What discourse strategies are used by leaders to perform leadership?

3. How does the team construct a group identity and what role do leaders play in this?

While leadership is the central theme of these research questions, this theme has been addressed using two complementary analytical approaches, both of which are used to a greater or lesser extent in answering all three research questions. The first of these is to view leadership as a communicative practice. The second is to view leadership as a component of identity.

The first approach is demonstrated in Chapter 4 which examines how the CofP model can be applied to the Prems, identifying the two positional groups (Forwards and Backs) as CofPs. Although forming around a factor imposed by the organisational structure inherent in rugby game-play, the players in these groups negotiate ways of belonging to their respective groups which are identifiable in their discourse. Moreover, the analysis of the communicative norms of each CofP suggests that the spatial 
organisation inherent in the different training and match-play practices are also influential in shaping the strategies used in their communication practices.

As well as the identification of the positional CofPs, Chapter 4 identifies the coaches as forming the core of another CofP, which takes leadership as its defining practice. In addition to the coaches, the institutionally reified player leaders (i.e. the members of the captaincy structure) can also be viewed as members of the Leadership CofP. Using the concept of trajectory, I discuss how some leaders align more closely with local leadership practice than others, thus positioning them closer to core status in this CofP. As time went on, other players displayed communicative practices that were aligned with the practices of this CofP, thus positioning them on an inward trajectory in the CofP. In other words, the emergence of new leaders was integrated into the CofP model. The concept of alignment also draws upon the idea of leadership as identity, by linking the alignment and negotiation of communicative practices with the construction of membership-based identity. Thus, Chapter 4 provides an answer to the first research question; members of the rugby team construct themselves as leaders by aligning their communicative practices to those of the coaches, who are institutionally defined as the leaders of the team and form the core of the Leadership CofP. Leaders are not brokers between CofPs, in fact this research shows that in a solidarity focused organisation they can be (and frequently are) core members of multiple CofPs.

Chapter 5 focuses on the Leadership CofP in greater detail, examining the discourse strategies used to enact leadership. In this regard it answers the second research question by taking leadership practice as a starting point and examining the different stances that the coaches and captains take in the course of practising leadership. In broad terms, the leadership strategy that is effectively used in the Prems is a distributed form of leadership, with each leader performing a complementary role in enacting leadership. However it is the analysis of leadership as a spatialised practice that is perhaps the most important contribution of Chapter 5. Viewing leadership as a practice that is negotiated back-stage and performed front-stage, and in which each leader takes a different stance, indexing and constructing a leadership identity, synthesises leadership practice and identity via stance. The examples presented in Chapter 5 show linked back-stage and front-stage interactions between the two coaches which demonstrate three key points regarding their leadership discourse. Firstly they 
construct themselves as equals in the leadership of the team through a narrative which constructs a group boundary between them and one of the other coaches in the club. Secondly they align their leadership practice by negotiating back-stage the stance towards the players that each will take front-stage. Thirdly, they perform their complementary "good cop/bad cop" stances frontstage with Parky admonishing players for their poor attitude while Tommo encourages them to try harder next time.

The third research question is addressed in Chapter 6, which discusses how stance and indexicality use global ideologies of rugby to construct local team identity. In examining team identity it looks at territorial ideologies, and ideologies of masculinity that are routinely indexed in the pre-match construction of team identity. This extends the discussion of front-stage and back-stage discursive spaces and shows that the leaders play an important part in the construction of team identity in front-stage events, partly because they are the speakers who contribute the most speech. It is however the discussion of rugby ideology that differentiates this chapter from those that precede it. In incorporating ideology, Chapter 6 demonstrates how global, or macro-level identities are renegotiated through locally constructed stances. Thus, while Chapter 4 could be said to be primarily based on the "practice" view of leadership, and Chapter 5 a mix of practice and identity, Chapter 6 is predominantly concerned with the construction of a team identity. In doing so it lays the foundations for Chapter 7 , which, by looking at the development of practices and cumulative stances over time, discusses the role of leaders in creating team identity. This is shown by the role that leaders play in ritual communicative events such as the huddle or team meeting. In addition, Chapter 7 demonstrates what happens when a core leader leaves the team, i.e. how leadership practice and the performance of ritual events are renegotiated by official and emergent members of the Leadership CofP. Thus the thesis returns to the negotiation of practice, suggesting that by constructing themselves as leaders through alignment of existing practice, members of the team have the opportunity to renegotiate these leadership practices.

\subsection{Methodological implications}

As Chapters 2 and 3 clearly express, this research is firmly grounded in ethnography. Not only has it been used as a methodology with which to access and record authentic interactions, it has also been central to the interpretation of these interactions. While 
the exact way in which ethnography is conducted is unique to every researcher (Agar 1996), this research has followed principles of ethnographic method arising from sociolinguistics and anthropology, and applied them to the study of a sports team. In doing so, an approach that utilised my own background in rugby combined with my status as a non-New Zealander was developed which allowed me to position myself as both "native" (of the sport) and "non-native" (of the culture). This gave me both the access to inside information only available to a trusted insider, but also the dispensation to ask questions concerning events, interactions or aspects of culture about which knowledge may have been implicit for a rugby playing New Zealander.

In addition to the general methodology of ethnography, a practical methodological outcome of this research has been the way in which audio recordings were collected. Recording players presented a methodological challenge which was overcome using a consultative approach, resulting in use of recording devices that could be worn by players during training sessions and their subsequent multi-tracking. Consequently, having the ability to add or remove microphone inputs when listen to an interaction proved an extremely useful resource for analysing the spoken data and aided considerably in the process of identifying communicative events that could be fully transcribed. Although the technology used was rudimentary due to budgetary limitations, the rigorous testing that was involved suggested ways in which this could be further developed, with potential applications that will be described in the penultimate section of this chapter.

\subsection{Theoretical implications}

\subsubsection{Identity}

The idea that identity is both performed and constructed through linguistic practice is widely accepted among sociolinguists; this research adds to existing work on the synthesis of the concepts of leadership practice, identity and performance. Where my research differs is the way in which leadership has been conceptualised as a spatialised practice through which identity is constructed. This builds on analyses of relationship between discourse and space originating from sociology (e.g. Alexander 2004; Hochschild 2010; Johnstone 2010; Lefebvre 1991), human geography (Gregson and Rose 2000; Simonsen 2007; Sommer 1961; Werlen 1993), and multimodal discourse 


\section{N. A. Wilson}

analysis (Mondada 2009). It is however, ultimately grounded in the data, following the grounded theory approach espoused in this research. The crux of the argument regarding spatialisation of discourse in the rugby team is found in the application of the terms front-stage and back-stage to describe the different conceptual spaces in which events with different identity and leadership functions take place. My use of these terms extends their prior use by Goffman (1959) and Richards (Richards 2006, 2010), and thus, in my view, makes a valuable contribution to research on interaction in organisations.

Every communicative event that takes place within the team can be viewed as constructing team identity. Furthermore, multiple team identities can be called upon that are appropriate for different situations and organisational goals. Rugby team identity is essentially a record of negotiated local practices embedded in cultural ideologies. Therefore it might be said that team identity is constructed through renegotiating the ideologies that define rugby at a level specific to the team and that further the goals of the team. This renegotiation is steered by the coaches who not only reify other leaders, but provide the initial move in the negotiation of leadership practice and team identity by performing the first leadership discourse in the Prems. This positions the coaches at the core of the leadership structure which forms a CofP with the addition of other members and the negotiation of leadership practice. At a more micro level, this is achieved by indexing local stances, global ideologies and macro-level identities through the combination of a number of discourse features and strategies such as swearing, repetition, formulaic language, delineating group boundaries, jocular insults, co-operation, competitiveness and territoriality. This indexing and local negotiation of global rugby ideologies is an important contribution that this research makes to the study of identity in discourse.

\subsubsection{Communities of Practice}

By describing the evolving communicative practices of the CofPs in the Prems at different points in the season, this thesis has attempted to build a picture of the Prems as a CofP in which practice is steered by the leaders but is always up for negotiation. This shows how CofPs that start as institutionally defined groups can negotiate practices and identities by the way they interact. While these practices and identities may be viewed as stable for a time, they can be renegotiated when necessary. Studying a 
system of CofPs from their inception to their conclusion gives a very different picture of the CofP than is found by describing the practice of an existing CofP which may appear to change only in terms of individual trajectories towards or away from the (apparently fixed) core of a CofP. An alternative would be, rather than viewing individual movement within a fixed CofP, to view the core of a CofP (as a collection of individuals with shared practices that are most aligned with the enterprise of the CofP) as a shifting centre, being pulled in different directions over a plane of practice. However this is only possible if we analyse a CofP in its temporally situated context.

\subsubsection{Leadership}

In addition to the general contribution this thesis makes to CofP theory, it shows that the CofP model can be used to analyse the way in which individuals construct themselves as potential leaders through their performance of locally defined leadership practices. Thus the CofP can be used as a framework for analysing leader emergence, an application to which it has not yet been put. Additionally, this thesis has explored the use of distributed leadership strategies, a topic in which there has been growing interest among researchers of leadership discourse in recent years (Vine et al. 2008). This thesis builds on this research, looking not only how co-leaders perform in frontstage interactions, when they are enacting leadership roles, but also in back-stage interactions when they negotiate these roles with each other..

By viewing leadership as being grounded in social (specifically linguistic) practice (Bolden 2011; Crevani et al. 2010; Drath et al. 2008; Raelin 2011) and team identity the same (Bauman 2000; Bucholtz and Hall 2005; Moore 2010a; Mullany 2010; Wenger 1998a), we can see that they are closely related. Leadership has been linked to identity through stance, in that adopting particular discourse features and strategies indexes a leader's membership of CofPs within the team, potentially lessening or increasing the distance between them and the players. As identity has been described as the record of stance over time (Jaffe 2009), it may be extrapolated that team identity includes every leadership stance that has been taken in the team. This can also be described in CofP terms as the repertoire of the Leadership CofP. Team identity is thus allied to the concept of Communities of Practice and the team can be viewed as a CofP which combines the repertoires of its constituent CofPs. 
Leadership in the Prems may thus be said to be the successful negotiation of team identity that serves the organisational goals of the team. Leadership styles, or stances, are dependent on the CofP membership of the leader, the event in which they perform leadership and the endorsement of their stance as a leader by the players. Each leader has a number of different leadership styles he can employ and may do so in concert with another leader, thus enacting distributed leadership (Bolden 2011), which is the basis for the postulation that there exists a Leadership CofP. This is also based on the idea that leadership is a form of practice, that it is practice with purpose (Raelin 2011).

While this section has highlighted the theoretical implications of this research, in keeping with the consultative nature of Linguistic Ethnography described in Chapters 2 and 3, it is important to also address the implications for the practitioners of sport: the coaches and players. The following section summarises how the inclusion of the players and coaches was a consideration at each stage of the research.

\subsection{Implications for sports leadership}

In doing research with rather than research on (Cameron et al. 1992), the involvement of participants is crucial not only in the data collection methodology but in shaping analysis and being informed of findings. Chapter 3 described how players were consulted and helped test the recording equipment. A further way in which players and coaches were involved in the research (beyond simply being the subjects of ethnography and recording) is in the follow-up contact I had with them. This took the form of reports and interviews with the coaches and a feedback workshop with the players.

A possible application of this research to team sport is that discourse analysis can be used as a tool for player and coach self-development. This has been demonstrated firstly through the use of feedback reports that were given to the coaches, which influenced the way in which they organised leadership in the season following this research. Secondly, a feedback workshop was run with players, in which they were given some of the analytical tools that were used to examine their leadership discourse. This gave them a resource from which they might be able to expand their leadership skills. Given the extent to which performance analysis is already used in many areas of sports coaching, this would seem a logical area for further development and could have 
particular benefits for teams that have a goal of player development, such as age-level representative teams.

A discourse analytic approach to sports team leadership could enhance existing training methods. Currently the majority of research that focuses on sports leadership takes a psychological approach (e.g. Gordon 2007; Gourley 2003; Jambor and Zhang 1997) or an organisational management approach (e.g. Gilbertson, Blyde, Gianotti, Gilbertson, et al. 2006). It may be beneficial to the development of coaching development resources to complement this with language-focused methods of improving coaches' leadership skills. Given the reaction to the research reports compiled for the coaches in this team, this is an approach that would certainly be of interest to them. However, a detailed test of language-focused coach development would be necessary in order to discover the scope of the possible benefits.

This is one possible area in which this thesis could prompt further research; the following section suggests some other possible directions for future research.

\subsection{Future avenues of research}

Given the paucity of linguistic research on sports team discourse, the potential areas for investigation are numerous. The next step in this research could be to expand the focus to amateur teams in other sports, or professional teams in rugby, for the purposes of comparison. Alternatively, the idea of global rugby ideologies discussed in Chapters 6 and 7 could be further investigated by comparing local practices in rugby teams in different countries and cultures.

However, there is also great potential for analysing the data collected in the course of this research from other angles. One area that I feel could be explored further is the discursive construction of masculinity in the team. This would follow the extensive tradition of sociological research in sports masculinities (e.g. Adams et al. 2010; Clayton and Humberstone 2006; Light and Kirk 2000; Messner 1995; Nauright and Chandler 1996) but with a more detailed focus on language. Another possibility would be further analysis of the way in which compliments and criticism function in team discourse, which would add to the existing socio-pragmatic research that deals with these functions of discourse (e.g. Ervin-Tripp 1976; Herbert and Straight 1989; Holmes 1986b, 1998a; Vine 2004). 
As noted in the discussion of the methodological implications of this research, the recording strategies that were devised could also be further developed. Recent sports performance research suggests that the use of GPS trackers on rugby players while training could provide useful information regarding the efficiency of their running angles and their work-rate (Cunniffe, Proctor, Baker and Davies 2009). This could be integrated into a recording unit, which would give an absolute position for a player at every instant of a recording. What this would make possible is an animated graphical representation of the position of every player during a training session, with microphone feeds from each player able to be turned on or off by selecting the player at any point in their movement, thus allowing speech events that take place on various parts of the pitch to be identified with ease. The application of this would not be limited to rugby or sport, however; any recording situation involving multiple speakers who routinely move around frequently taking part in numerous interactional encounters could benefit from this. However, such an application of this data collection methodology would not be a substitute for ethnography, merely an additional resource in the researcher's analytical toolkit.

\subsection{Closing remarks}

Perhaps due to its ethnographic nature, this research has covered a wide range of topics, and this has been necessary in order to give as full a picture as possible of the rugby team. As the above section on future research shows, there are many topics that I hope to revisit and examine further. The final conclusion of this research, however, is that leadership in the Prems is enacted through a range of leadership strategies within a distributed leadership framework. Leaders co-operate with one other and their followers to negotiate a way of enacting leadership that is effective for the team. This draws on team identity, the repetition of events over the course of the season and the juxtaposition of front and back-stage events in which leadership can be both constructed and enacted. It also utilises the unique practices that form the communicative repertoire of each positional CofP and combines all of these aspects of leadership into a repertoire of leadership discourse.

Through the close ethnography and recording of an amateur rugby team in New Zealand, my thesis has shown how leadership identities are constructed through leadership practice, and demonstrated that in rugby, team identity is created in the 
intersection of local practice and ideology. Rugby team leadership does not begin and end with the rugby coach, but is distributed across a range of individuals within the team and players can emerge as leaders through their performance of discourse that follows local leadership practice. Thus discourse is not just the means by which leaders enact leadership, it is also how they become leaders. 
N. A. Wilson 


\section{References}

Adams, Adi, Eric Anderson and Mark McCormack. 2010. Establishing and challenging masculinity: The influence of gendered discourses in organized sport. Journal of Language and Social Psychology 29(3): 278-300.

Agar, Michael. 1986. Speaking of ethnography, Beverly Hills, California: Sage Publications.

Agar, Michael. 1996. The professional stranger: an informal introduction to ethnography 2nd ed., San Diego, California: Academic Press.

Agha, Asif. 2005. Voice, Footing, Enregisterment. Journal of Linguistic Anthropology 15(1): 38-59.

Agho, Augustine 0. 2009. Perspectives of Senior-Level Executives on Effective Followership and Leadership. Journal of Leadership and Organizational Studies 16(2): 159-166.

Aijmer, Karin. 2002. English discourse particles: Evidence from a corpus, Amsterdam, The Netherlands: John Benjamins.

Alexander, Jeffrey C. 2004. Cultural pragmatics: Social performance between ritual and strategy. Sociological Theory 22(4): 527-573.

Allan, George. 2003. A critique of using grounded theory as a research method. Electronic Journal of Business Research Methods 2(1): 1-10.

Allan, Keith. 2010. Referring as a pragmatic act. Journal of Pragmatics 42(11): 29192931.

Alvesson, Mats and Stefan Sveningsson. 2003. Managers doing leadership: The extraordinarization of the mundane. Human Relations 56(12): 1435-1459.

Anderson, Eric and Rhidian McGuire. 2010. Inclusive masculinity theory and the gendered politics of men's rugby. Journal of Gender Studies 19(3): 249-261.

Anderson, Eric. 2005. Orthodox and inclusive masculinity: Competing masculinities among heterosexual men in a feminized terrain. Sociological Perspectives 48(3): 337.

Anderson, Eric. 2007. Developing a coaching identity. In Jim Denison (ed.), Coaching knowledges: Understanding the dynamics of sport performance. London: A \& C Black.

Anderson, Eric. 2008. "I used to think women were weak": Orthodox masculinity, gender segregation, and sport. Sociological Forum 23(2): 257-280.

Anderson, Eric. 2009. Inclusive masculinity: The changing nature of masculinities, New York; Abingdon, U.K.: Routledge.

Atkinson, Paul and Martyn Hammersley. 1994. Ethnography and participant observation. In Norma. K. Denzin and Yvonne S. Lincoln (eds.), Handbook of qualitative research, 248-261. London; Thousand Oaks, California: Sage.

Auld, Christopher and Graham Cuskelly. 2006. Organisational theory and sport management. In Sarah Leberman, Chris Collins, and Linda Trenberth (eds.), Sport business management in Aotearoa/New Zealand. Melbourne, Australia: Thomson Dunmore Press.

Austin, John Langshaw. 1975. How to do things with words 2nd ed. J. O. Urmson and Marina Sbisa (eds.), Oxford, U.K.: Clarendon Press.

Avolio, Bruce J., Fred O. Walumbwa and Todd J. Weber. 2009. Leadership: Current theories, research, and future directions. Annual Review of Psychology 60(1): 421-449. 
Bailey, Benjamin. 2008. Interactional sociolinguistics. In Wolfgang Donsbach (ed.), International Encyclopedia of Communication, 2314-2318. Malden, Massachusets: Blackwell.

Bailey, Lee Ann and Lenora A. Timm. 1976. More on women's — and men's Expletives. Anthropological Linguistics 18(9): 438-449.

Baker, Paul. 2006. Using corpora in discourse analysis, London; New York: Continuum International Publishing Group.

Bale, John. 2001. Sport, space, and the city, Caldwell, New Jersey: Blackburn Press.

Bass, Bernard M. 1990. Bass and Stodgill's handbook of leadership: Theory, research, and managerial applications 3rd ed., New York; London; Toronto; Sydney; Singapore: The Free Press.

Bauman, R. 2000. Language, identity, performance. Pragmatics 10(1): 1-6.

Baxter, Judith and Kieran Wallace. 2009. Outside in-group and out-group identities? Constructing male solidarity and female exclusion in UK builders' talk. Discourse \& Society 20(4): 411-429.

Baxter, Judith. 2010. The language of female leadership, Basingstoke, U.K.; New York: Palgrave Macmillan.

Bayard, Donn and Sateesh Krishnayya. 2001. Gender, expletive use, and context: Male and female expletive use in structured and unstructured conversation among New Zealand university students. Women and Language 24(1): 1-15.

BBC Sport. 2009. Gunners lack leadership - Toure.

Becker, Kara. 2009. /r/ and the construction of place identity on New York City's Lower East Side. Journal of Sociolinguistics 13(5): 634-658.

Bell, Allan. 1984. Language style as audience design. Language in Society 13(2): 145204.

Bell, Allan. 1999. Styling the other to define the self: A study in New Zealand identity making. Journal of Sociolinguistics 3(4): 523-541.

Bell, Allan. 2000. Maori and Pakeha English: A case study. In Allan Bell and Koenraad Kuiper (eds.), New Zealand English, 221-248. Wellington, New Zealand: Victoria University Press.

Bell, Allan. 2007. Style in dialogue: Bakhtin and sociolinguistic theory. In Robert Bayley and Ceil Lucas (eds.), Sociolinguistic variation: Theories, methods, and applications, 90-109. Cambridge, U.K.: Cambridge University Press.

Binns, Jennifer. 2008. The ethics of relational leading: Gender matters. Gender, Work \& Organization 15(6): 600-620.

Birrell, Susan. 1981. Sport as ritual: Interpretations from Durkheim to Goffman. Social Forces 60(2): 354-376.

Blommaert, Jan. 2005. Discourse: A critical introduction, Cambridge, U.K.: Cambridge University Press.

Blommaert, Jan. 2007. On scope and depth in linguistic ethnography. Journal of Sociolinguistics 11(5): 682-688.

Boeije, Hennie. 2010. Analysis in qualitative research, Los Angeles: SAGE.

Bolden, Galina B. 2006. Little words that matter: Discourse markers "so" and "oh" and the doing of other-attentiveness in social interaction. Journal of Communication 56(4): 661-688.

Bolden, Richard. 2011. Distributed leadership in organizations: A review of theory and research. International Journal of Management Reviews. 
Bono, Joyce E. and Timothy A. Judge. 2004. Personality and transformational and transactional leadership: A meta-analysis. Journal of Applied Psychology 89(5): 901-910.

Boxer, Diana. 2002. Applying sociolinguistics: Domains and face-to-face interaction, Amsterdam; Philadelphia: John Benjamins Pub. Co.

Brannan, Matthew J. 2007. Sexuality, gender and legitimate peripheral participation. In Jason Hughes, Nick Jewson, and Lorna Unwin (eds.), Communities of Practice: Critical perspectives, 120-130. London: Routledge.

Brown, John Seely and Paul Duguid. 1991. Organizational learning and communities-ofpractice: Toward a unified view of working, learning, and innovation. Organization Science 2(1): 40-57.

Bryson, Lois. 1987. Sport and the maintenance of masculine hegemony. Women's Studies International Forum 10(4): 349-360.

Bucholtz, Mary and Kira Hall. 2004. Theorizing identity in language and sexuality research. Language in Society 33(04): 469-515.

Bucholtz, Mary and Kira Hall. 2005. Identity and interaction: A sociocultural linguistic approach. Discourse Studies 7(4-5): 585-614.

Bucholtz, Mary and Kira Hall. 2008a. All of the above: New coalitions in sociocultural linguistics. Journal of Sociolinguistics 12(4): 401-431.

Bucholtz, Mary and Kira Hall. 2008b. Finding identity: Theory and data. Multilingua Journal of Cross-Cultural and Interlanguage Communication 27(1-2): 151-163.

Bucholtz, Mary and Kira Hall. 2010. Locating identity in language. In Carmen Llamas and Dominic Watt (eds.), Language and identities, 18-28. Edinburgh: Edinburgh University Press.

Bucholtz, Mary. 1999. "Why be normal?": Language and identity practices in a community of nerd girls. Language in Society 28(2): 203-223.

Busher, Hugh, Linda Hammersley-Fletcher and Chris Turner. 2007. Making sense of middle leadership: Community, power and practice. School Leadership \& Management 27(5): 405-422.

Butler, Judith. 1997. Excitable speech: A politics of the performative, New York; London: Routledge.

Cameron, Deborah, Elizabeth Frazer, Penelope Harvey, Ben Rampton and Kay Richardson. 1992. Researching language: Issues of power and method, London; New York: Routledge.

Cameron, Deborah. 1997. Performing gender identity: Young men's talk and the construction of heterosexual masculinity. In Sally Johnson and Ulrike Hanna Meinhof (eds.), Language and masculinity. Oxford, U.K.: Blackwell.

Cameron, Deborah. 2008. Gender and Language Ideologies. In Janet Holmes and Miriam Meyerhoff (eds.), The Handbook of Language and Gender, 447-467. WileyBlackwell.

Campbell, Lorne, Jeffry A. Simpson, Mark Stewart and John Manning. 2003. Putting personality in social context: Extraversion, emergent leadership, and the availability of rewards. Personal Social Psychology Bulletin 29(12): 1547-1559.

Carotenuto, L., W. Etienne, M. Fontaine, J. Friedman, H. Newberg, M. Muller, M. Simpson, J. Slusher and K. Stevenson. 1999. CommunitySpace: Toward flexible support for voluntary knowledge communities. Paper presented at Changing Places Workshop, 1999, at London. Changing Places Workshop, London.

Chan, Angela. 2007. Same context, different strategies: A company director's discourse in business meetings. Journal of Asian Pacific Communication 17: 61-81. 
Chandler, Timothy John Lindsay. 1996. The development of rugby football, 1830 - 1880. In John Nauright and Timothy John Lindsay Chandler (eds.), Making men: Rugby and masculine identity. London: Frank Cass \& Co.

Charmaz, Kathy and Richard G. Mitchell. 2001. Grounded theory in ethnography. In Paul Atkinson, Amanda Coffey, Sara Delamont, John Lofland, and Lyn Lofland (eds.), Handbook of ethnography, 160-174. London: Sage.

Charmaz, Kathy. 2001. Qualitative interviewing and grounded theory analysis. In Jaber F. Gubrium and James A. Holstein (eds.), Handbook of interview research: Context and method, 675-694. Thousand Oaks, California: Sage.

Chiles, Tina. 2007. The construction of an identity as "mentor" in white collar and academic workplaces: A preliminary analysis. Journal of Pragmatics 39(4): 730741.

Clayton, Ben and Barbara Humberstone. 2006. Men's talk: A (pro)feminist analysis of male university football players' discourse. International Review for the Sociology of Sport 41(3-4): 295-316.

Clift, Rebecca. 2006. Indexing stance: Reported speech as an interactional evidential. Journal of Sociolinguistics 10(5): 569-595.

Coates, Jennifer. 2003. Men talk: Stories in the making of masculinities, Oxford, U.K.: Blackwell.

Connell, Raewyn. 1987. Gender and power: Society, the person and sexual politics, Sydney, Australia: Allen \& Unwin.

Connell, Raewyn. 2005. Masculinities 2nd ed., Berkeley, California: University of California Press.

Copeland, Barry W. and Kathy Wida. 1996. Resolving team conflict: Coaching strategies to prevent player conflicts. JOPERD--The Journal of Physical Education, Recreation \& Dance 67(4): 52-54.

Corder, Saskia. 2004. Negotiating and performing gender role expectations through discourse: A study of the community of practice of a female football team. MA dissertation. Edinburgh, U.K.: University of Edinburgh.

Coupland, Nikolas. 2007. Style: Language variation and identity, Cambridge, U.K.: Cambridge University Press.

Crawford, Scott A.G.M. 1985. The game of "glory and hard knocks": A study of the interpenetration of rugby and New Zealand society. The Journal of Popular Culture 19(2): 77-92.

Creese, Angela. 2005. Mediating allegations of racism in a multiethnic London school. In David Barton and Karin Tusting (eds.), Beyond communities of practice: Language, power and social context, 55. Cambridge: Cambridge University Press.

Creese, Angela. 2010. Linguistic Ethnography. In Lia Litosseliti (ed.), Research methods in linguistics, 138-154. London; New York: Continuum.

Crevani, Lucia, Monica Lindgren and Johann Packendorff. 2010. Leadership, not leaders: On the study of leadership as practices and interactions. Scandinavian Journal of Management 26(1): 77-86.

Cunniffe, B., W. Proctor, J. S Baker and B. Davies. 2009. An evaluation of the physiological demands of elite rugby union using global positioning system tracking software. The Journal of Strength \& Conditioning Research 23(4): 1195.

Curry, Timothy Jon. 1991. Fraternal bonding in the locker room: A profeminist analysis of talk about competition and women. Sociology of Sport Journal 8(2): 119-135.

D’Arcy, Alexandra. 2010. Quoting ethnicity: Constructing dialogue in Aotearoa/New Zealand. Journal of Sociolinguistics 14(1): 60-88. 
Daly, Nicola, Janet Holmes, Jonathan Newton and Maria Stubbe. 2004. Expletives as solidarity signals in FTAs on the factory floor. Journal of Pragmatics 36(5): 945964.

Davies, Bethan. 2005. Communities of practice: Legitimacy not choice. Journal of Sociolinguistics 9(4): 557-581.

Dekker, Paul. 2002. Pronouns in a pragmatic semantics. Journal of Pragmatics 34(7): 815-827.

Delin, Judy. 2001. Keeping in step: Task structure, discourse structure, and utterance interpretation in the step aerobics workout. Discourse Processes 31(1): 61-89.

van Dijk, Teun. 2008. Discourse and context: A sociocognitive approach, Cambridge, U.K.: Cambridge University Press.

Donnelly, Peter and Kevin M. Young. 1985. Reproduction and transformation of cultural forms in sport: A contextual analysis of rugby. International Review for the Sociology of Sport 20(1-2): 19-38.

Drath, Wilfred H. and Charles J. Palus. 1994. Making common sense: Leadership as meaning-making in a community of practice, Greensboro, North Carolina: Center for Creative Leadership.

Drath, Wilfred H., Cynthia D. McCauley, Charles J. Palus, Ellen Van Velsor, Patricia M.G. O'Connor and John B. McGuire. 2008. Direction, alignment, commitment: Toward a more integrative ontology of leadership. The Leadership Quarterly 19(6): 635653.

DuBois, John. 2007. The stance triangle. In Robert Englebretson (ed.), Stancetaking in discourse: Subjectivity, evaluation, interaction, 139-182. Amsterdam, The Netherlands: John Benjamins.

Dunning, Eric G. 1986. Sport as a male preserve: Notes on the social sources of masculine identity and its transformations. Theory Culture Society 3(1): 79-90.

Duszak, Anna. (ed.) 2002. Us and others: Social identities across languages, discourses, and cultures, Amsterdam, The Netherlands: John Benjamins.

Eagly, Alice H and Mary C Johannesen-Schmidt. 2001. The leadership styles of women and men. Journal of Social Issues 57(4): 781-797.

Eckert, Penelope and Sally McConnell-Ginet. 1992. Think practically and look locally: Language and gender as community-based practice. Annual Review of Anthropology 21(1): 461-488.

Eckert, Penelope and Etienne Wenger. 2005. Communities of practice in sociolinguistics. Journal of Sociolinguistics 9(4): 582-589.

Eckert, Penelope. 1988. Adolescent social structure and the spread of linguistic change. Language in Society 17(2): 183-207.

Eckert, Penelope. 2000. Linguistic variation as social practice: The linguistic construction of identity in Belten High, Malden, Massachusetts; Oxford, U.K.: Blackwell.

Edge, Julian and Keith Richards. 1998. May I see your warrant, please? Justifying outcomes in qualitative research. Applied Linguistics 19(3): 334 -356.

Emmison, M. 1987. Victors and vanquished: The social organization of ceremonial congratulations and commiserations. Language \& Communication 7(2): 93-110.

Emmison, Mike. 1988. On the Interactional Management of Defeat. Sociology 22(2): 233 $-251$.

Ensink, Titus. 2003. The frame analysis of research interviews: Social categorization and footing in interview discourse. In Harry van den Berg, Margaret Wetherell, and Hanneke Houtkoop-Steenstra (eds.), Analyzing race talk, 156-177. Cambridge, U.K.: Cambridge University Press. 
Ervin-Tripp, Susan. 1976. Is Sybil there? The structure of some American English directives. Language in Society 5(1): 25-66.

Fairhurst, Gail T. 2008. Discursive leadership: A communication alternative to leadership psychology. Management Communication Quarterly 21(4): 510-521.

Falcous, Mark. 2007. The decolonizing national imaginary: Promotional media constructions during the 2005 Lions tour of Aotearoa New Zealand. Journal of Sport and Social Issues 31(4): 374-393.

Fasulo, Alessandra and Cristina Zucchermaglio. 2002. My selves and I: Identity markers in work meeting talk. Journal of Pragmatics 34(9): 1119-1144.

Feagin, Crawford. 2002. Entering the community: Fieldwork. In J. K. Chambers, Peter Trudgill, and Natalie Schilling-Estes (eds.), The handbook of language variation and change, 20-40. Malden, Massachussets: Blackwell Publishers.

Fenton, Lindsay T and Robert Pitter. 2010. Keeping the body in play: Pain, injury, and socialization in male rugby. Research Quarterly for Exercise and Sport 81(2): 212223.

Ferguson, Graeme W. 2004. You'll be a man if you play rugby: Sport and the construction of gender, Palmerston North, N.Z.: Dunmore Press in association with the Christchurch College of Education and Whitireia Publishing.

File, Kieran. forthcoming. Talking sport! The post match media interview as a discourse genre: A genre and register analysis. PhD Thesis. Wellington, New Zealand: Victoria University of Wellington.

FitzSimons, Peter. 1996. The rugby war, Sydney, Australia: HarperSports.

Fletcher, Joyce K. and Katrin Kaufer. 2003. Shared leadership. In Craig L. Pearce and Jay Alden Conger (eds.), Shared leadership: Reframing the hows and whys of leadership, 21-47.

Fletcher, Joyce K. 1999. Disappearing acts: Gender, power and relational practice at work, Cambridge, Massachussetts: MIT Press.

Fortanet, Inmaculada. 2004. The use of "we" in university lectures: Reference and function. English for Specific Purposes 23(1): 45-66.

Frohlick, Susan. 2005. That playfulness of white masculinity. Tourist Studies 5(2): 175.

Garrot-Lavoué, Élise. 2009. Interconnection of communities of practice: A web platform for knowledge management. Proceedings of the international conference on knowledge management and information sharing.

Gee, James Paul. 2005. Meaning making, communities of practice, and analytical toolkits. Journal of Sociolinguistics 9(4): 590-594.

Geyer, Naomi. 2009. Teasing and ambivalent face in Japanese multi-party discourse. Journal of Pragmatics 42(8): 2120-2130.

Gilbertson, Dai, Peter Blyde, Simon Gianotti, Deb Gilbertson and Dane Dougan. 2006. Leadership in sports organisations. In Sarah Leberman, Chris Collins, and Linda Trenberth (eds.), Sport business management in Aotearoa/New Zealand. Melbourne: Thomson Dunmore Press.

Giles, Howard, Justine Coupland and Nikolas Coupland. 1991. Contexts of accommodation: Developments in applied sociolinguistics, Cambridge, U.K.: Cambridge University Press.

Glaser, Barney G. and Anselm L. Strauss. 1967. The discovery of grounded theory: Strategies for qualitative research, Piscataway, New Jersey: Transaction Publishers.

Goffman, Erving. 1959. The presentation of self in everyday life, Harmondsworth, U.K.: Penguin. 
Goffman, Erving. 1967. Interaction ritual: Essays on face-to-face behaviour, London: Allen Lane.

Goffman, Erving. 1974. Frame analysis: An essay on the organization of experience, Cambridge, Massachussetts: Harvard University Press.

Goleman, Daniel. 2000. Leadership that gets results. Harvard Business Review 78(2): 7890.

Goodger, John. 1986. Ritual solidarity and sport. Acta Sociologica 29(3): 219 -224.

Goodwin, Charles. 2000. Action and embodiment within situated human interaction. Journal of Pragmatics 32(10): 1489-1522.

Gordon, Sandy. 2007. Sport and business coaching: Perspective of a sport psychologist. Australian Psychologist 42(4): 271 - 282.

Gourley, Michael. 2003. The psychological edge in rugby: Leadership and motivation in coaching team sports, Auckland, New Zealand: Human Synergistics.

Grainger, Andrew. 2009. Rugby, Pacific peoples, and the cultural politics of national identity in New Zealand. International Journal of the History of Sport 26(16): 2335-2357.

Gregson, N. and G. Rose. 2000. Taking Butler elsewhere: Performativities, spatialities and subjectivities. Environment and Planning D: Society and Space 18: 433-452.

Griffin, Larry J. 1992. Temporality, events, and explanation in historical sociology. Sociological Methods \& Research 20(4): 403 -427.

Grint, Keith. 2010. The sacred in leadership: Separation, sacrifice and silence. Organization Studies 31(1): 89-107.

Groves, Kevin S. 2005. Linking leader skills, follower attitudes, and contextual variables via an integrated model of charismatic leadership. Journal of Management 31(2): 255-277.

Gruneau, Richard. 2006. "Amateurism” as a sociological problem: Some reflections inspired by Eric Dunning. Sport in Society 9(4): 559-582.

Gumperz, John Joseph and Jenny Cook-Gumperz. 2008. Studying language, culture, and society: Sociolinguistics or linguistic anthropology?1. Journal of Sociolinguistics 12(4): 532-545.

Gumperz, John Joseph and Dell H. Hymes. 1972. The ethnography of communication, New York: Holt.

Gumperz, John Joseph. 1982a. Discourse strategies, Cambridge University Press.

Gumperz, John Joseph. 1999. On interactional sociolinguistic method. In Srikant Sarangi and Celia Roberts (eds.), Talk, work and institutional order: Discourse in medical, mediation and management settings, 454-471. Berlin; New York: Mouton de Gruyter.

Gumperz, John Joseph. 2005. Interactional sociolinguistics: A personal perspective. In Deborah Schiffrin, Deborah Tannen, and Heidi E. Hamilton (eds.), The handbook of discourse analysis, 215-228. Malden, Massachussetts: Blackwell.

Gumperz, John Joseph. (ed.) 1982b. Language and social identity, Cambridge: Cambridge University Press.

Guttmann, Allen. 1978. From ritual to record: The nature of modern sports, New York: Columbia University Press.

Halberstam, Judith. 1998. Female masculinity, Duke University Press.

Hall, Kira and Mary Bucholtz. (eds.) 1995. Gender articulated: Language and the socially constructed self, New York: Routledge.

Hall, Kira. 1999. Performativity. Journal of Linguistic Anthropology 9(1-2): 184-187. 
Halone, Kelby K. and Lindsey J. Meân. 2010. Situating sport, language, and culture as a site for intellectual discussion. Journal of Language and Social Psychology 29(3): $386-396$.

Hammersley, Martyn and Paul Atkinson. 1983. Ethnography, principles in practice, London; New York: Tavistock.

Hammersley, Martyn. 2007. Reflections on linguistic ethnography. Journal of Sociolinguistics 11(5): 689-695.

Hargreaves, Jennifer. (ed.) 1982. Sport, culture, and ideology, London: Routledge.

Harres, Annette. 1998. "But basically you're feeling well, are you?" Tag questions in medical consultations. Health Communication 10(2): 111-123.

Harris, John. 2007. Doing gender on and off the pitch: The world of female football players. Sociological Research Online 12(1): http://www.socresonline.org.uk/12/1/harris.html.

Hay, Jennifer. 1994. Jocular abuse patterns in mixed-group interaction. Wellington Working Papers in Linguistics 6: 26-55.

Heath, Shirley Brice and Juliet Langman. 1994. Shared thinking and the register of coaching. In Douglas Biber and Edward Finegan (eds.), Sociolinguistic perspectives on register, 82-105. New York: Oxford University Press US.

Heenan, David A. and Warren Bennis. 1999. Co-Leaders: The Power of Great Partnerships, New York: John Wiley \& Sons.

Herbert, Robert K. and H. Stephen Straight. 1989. Compliment-rejection versus compliment-avoidance: Listener-based versus speaker-based pragmatic strategies. Language \& Communication 9(1): 35-47.

Heritage, John and Geoffrey Raymond. 2005. The terms of agreement: Indexing epistemic authority and subordination in talk-in-interaction. Social Psychology Quarterly 68(1): 15 -38.

Heritage, John. 1998. Oh-prefaced responses to inquiry. Language in Society 27(3): 291334.

Hochschild, Thomas R. 2010. "Our club": Place-work and the negotiation of collective belongingness. Journal of Contemporary Ethnography 39(6): 619 -645.

Hokowhitu, Brendan. 2003. "Physical beings": Stereotypes, sport and the "physical education" of New Zealand Māori. Sport in Society 6(2): 192-218.

Holmes, Janet and Allan Bell. 1988. Learning by experience: Notes for New Zealand social dialectologists. Te Reo 31: 19 - 49.

Holmes, Janet and Meredith Marra. 2002a. Having a laugh at work: How humour contributes to workplace culture. Journal of Pragmatics 34(12): 1683-1710.

Holmes, Janet and Meredith Marra. 2002b. Humour as a discursive boundary marker in social interaction. In Anna Duszak (ed.), Us and others: Social identities across languages, discourses, and cultures, 377-400. Amsterdam, The Netherlands: John Benjamins.

Holmes, Janet and Meredith Marra. 2004a. Leadership and managing conflict in meetings. Pragmatics 14(4): 439-462.

Holmes, Janet and Meredith Marra. 2004b. Relational practice in the workplace: Women's talk or gendered discourse? Language in Society 33(03): 377-398.

Holmes, Janet and Miriam Meyerhoff. 1999. The community of practice: Theories and methodologies in language and gender research. Language in Society 28(02): 173-183. 
Holmes, Janet and Stephanie Schnurr. 2005. Politeness, humor and gender in the workplace: Negotiating norms and identifying contestation. Journal of Politeness Research: Language, Behaviour, Culture 1(1): 121-150.

Holmes, Janet and Stephanie Schnurr. 2006. "Doing femininity" at work: More than just relational practice. Journal of Sociolinguistics 10(1): 31-51.

Holmes, Janet and Maria Stubbe. 1997. Good listeners: Gender differences in New Zealand conversation. Women and Language 20(2).

Holmes, Janet and Maria Stubbe. 2003a. Power and politeness in the workplace: $A$ sociolinguistic analysis of talk at work, Harlow, U.K.: Longman.

Holmes, Janet and Maria Stubbe. 2003b. "Feminine” workplaces: Stereotype and reality. In Janet Holmes and Miriam Meyerhoff (eds.), The handbook of language and gender, 572-599. Malden, Massachussetts: Blackwell.

Holmes, Janet, Meredith Marra and Bernadette Vine. 2011. Leadership, discourse, and ethnicity, Oxford, U.K.: Oxford University Press.

Holmes, Janet, Stephanie Schnurr, Angela Chan and Tina Chiles. 2003. The discourse of leadership. Te Reo 46: 31-46.

Holmes, Janet, Maria Stubbe and Bernadette Vine. 1999. Constructing professional identity: "Doing power" in policy units. In Srikant Sarangi and Celia Roberts (eds.), Talk, work and institutional order: Discourse in medical, mediation and management settings, 351-385. Berlin; New York: Mouton de Gruyter.

Holmes, Janet, Bernadette Vine and Meredith Marra. 2009. Maori men at work: Leadership, discourse, and ethnic identity. Intercultural Pragmatics 6(3): 345366.

Holmes, Janet. 1986a. Functions of “you know” in women's and men's speech. Language in Society 15(1): 1-22.

Holmes, Janet. 1986b. Compliments and compliment responses in New Zealand English. Anthropological Linguistics 28(4): 485-508.

Holmes, Janet. 1998a. Complimenting - a positive politeness strategy. In Jennifer Coates (ed.), Language and gender: A reader, 100-120. Oxford: Blackwell.

Holmes, Janet. 1998b. Narrative structure: Some contrasts between Maori and Pakeha story-telling. Multilingua - Journal of Cross-Cultural and Interlanguage Communication 17(1): 25-58.

Holmes, Janet. 2000. Victoria University of Wellington's Language in the Workplace Project: An overview. Language in the Workplace occasional papers 1: 1-18.

Holmes, Janet. 2005a. Story-telling at work: A complex discursive resource for integrating personal, professional and social identities. Discourse Studies 7(6): 671-700.

Holmes, Janet. 2005b. Leadership talk: How do leaders "do mentoring", and is gender relevant? Journal of Pragmatics 37(11): 1779-1800.

Holmes, Janet. 2005c. Using Māori English in New Zealand. International Journal of the Sociology of Language 172: 91-115.

Holmes, Janet. 2006. Gendered talk at work: Constructing social identity through workplace interaction, Malden, MA: Blackwell.

Holmes, Janet. 2008. Gendered discourse at work. Language and Linguistics Compass 2(3): 478-495.

Holmes, Janet. 2009. Men, masculinities and leadership: Different discourse styles at work. In Pia Pichler and Eva Eppler (eds.), Gender and spoken interaction, 186210. London: Palgrave Macmillan. 
Holt, Elizabeth. 2000. Reporting and reacting: Concurrent responses to reported speech. Research on Language \& Social Interaction 33(4): 425.

van Houtum, Henk and Frank van Dam. 2002. Topophilia or topoporno? Patriotic place attachment in international football derbies. HAGAR International Social Science Review 3(2): 231-248.

Howe, P. David. 2001. An ethnography of pain and injury in professional rugby union. International Review for the Sociology of Sport 36(3): 289.

Howells, Lloyd T and Selwyn W Becker. 1962. Seating arrangement and leadership emergence. Journal of Abnormal and Social Psychology 64(2): 148-150.

Hughes, Charlotte. 2005. Moira's lament? Feminist advocacy and the 1981 Springbok tour of New Zealand. In Greg Ryan (ed.), Tackling rugby myths: rugby and New Zealand society, 1854-2004, 137-150. Dunedin N.Z.: University of Otago Press.

Hughes, Jason, Nick Jewson and Lorna Unwin. (eds.) 2007. Communities of practice: Critical perspectives, London: Routledge.

Hymes, Dell. 2003. Foundations in sociolinguistics: An ethnographic approach, New York: Routledge.

Iacuone, David. 2005. "Real men are tough guys": Hegemonic masculinity and safety in the construction industry. The Journal of Men's Studies 13(2): 247-266.

Jackson, Brad and Eric Guthey. 2007. Putting the visual into the social construction of leadership. In Boas Shamir, Rajnandini Pillai, Michelle C. Bligh, and Mary UhlBien (eds.), Follower-centered perspectives on leadership: A tribute to the memory of James R. Meindl, 167-186. Charlotte, North Carolina: Information Age Publishing.

Jackson, Brad and Ken Parry. 2001. The hero manager: Learning from New Zealand's top chief executives, Auckland, New Zealand: Penguin.

Jaffe, Alexandra M. 2009. Introduction. In Alexandra M Jaffe (ed.), Stance: Sociolinguistic perspectives, 3-28. Oxford: Oxford University Press.

Jambor, E. A and J. J Zhang. 1997. Investigating leadership, gender, and coaching level using the Revised Leadership for Sport Scale. Journal of Sport Behaviour 20: 313321.

Jay, Timothy and Kristin Janschewitz. 2008. The pragmatics of swearing. Journal of Politeness Research. Language, Behaviour, Culture 4(2): 267-288.

Johnston, Lorraine and Shelley Robertson. 1993. "Hey yous!" the Maori-NZE interface in sociolinguistic rules of address. Te Reo 36: 115-127.

Johnstone, Barbara and Scott Fabius Kiesling. 2008. Indexicality and experience: Exploring the meaning of /aw/-monophongization in Pittsburgh. Journal of Sociolinguistics 12(1): 5-33.

Johnstone, Barbara. 2000. Qualitative methods in sociolinguistics, New York; Oxford, U.K.: Oxford University Press.

Johnstone, Barbara. 2008. Discourse analysis 2nd ed., Malden, Massachussetts: Blackwell.

Johnstone, Barbara. 2009. Stance, style, and the linguistic individual. In Alexandra M Jaffe (ed.), Stance: Sociolinguistic perspectives, 29-52. Oxford, U.K.: Oxford University Press.

Johnstone, Barbara. 2010. Indexing the Local. In Nikolas Coupland (ed.), The handbook of language and globalization, 386-405. Malden, Massachusetts; Oxford, U.K.: Wiley-Blackwell.

Kendall, Shari. 2004. Framing authority: Gender, face, and mitigation at a radio network. Discourse and Society 15(1): 55-79. 
Kennedy, Robert and Tania Zamuner. 2006. Nicknames and the Lexicon of Sports. American Speech 81(4): 387-422.

Kiesling, Scott Fabius. 1996. Men's identities and patterns of variation. University of Pennsylvania Working Papers in Linguistics: 171-196.

Kiesling, Scott Fabius. 1997. Power and the language of men. In Sally Johnson and Ulrike Hanna Meinhof (eds.), Language and masculinity. Oxford, U.K.: Blackwell.

Kiesling, Scott Fabius. 2001a. Stances of whiteness and hegemony in fraternity men's discourse. Journal of Linguistic Anthropology 11(1): 101-115.

Kiesling, Scott Fabius. 2001b. "Now I gotta watch what I say": Shifting constructions of masculinity in discourse. Journal of Linguistic Anthropology 11(2): 250-273.

Kiesling, Scott Fabius. 2004. Dude. American Speech 79(3): 281-305.

Kiesling, Scott Fabius. 2005. Homosocial desire in men's talk: Balancing and re-creating cultural discourses of masculinity. Language in Society 34(05): 695-726.

Kiesling, Scott Fabius. 2006. Hegemonic identity-making in narrative. In Anna De Fina, Deborah Schiffrin, and Michael Bamberg (eds.), Discourse and identity, 261-287. Cambridge, U.K.: Cambridge University Press.

Kiesling, Scott Fabius. 2007. Men, masculinities, and language. Language and Linguistics Compass 1(6): 653-673.

Kiesling, Scott Fabius. 2009. Style as stance. In Alexandra M Jaffe (ed.), Stance: Sociolinguistic perspectives, 171. Oxford, U.K.: Oxford University Press.

King, Brian W. 2011. Linguistic negotiations of sexual agency in sexuality education. PhD Thesis. Wellington, New Zealand: Victoria University of Wellington.

De Klerk, Vivian. 1997. The role of expletives in the construction of masculinity. In, Language and masculinity, 144-58. Oxford, U.K.: Blackwell.

Komives, Susan R., Susan D. Longerbeam, Julie E. Owen, Felicia C. Mainella and Laura. Osteen. 2006. A leadership identity development model: Applications from a grounded theory. Journal of College Student Development 47(4): 401-418.

Komives, Susan R., Julie E. Owen, Susan D. Longerbeam, Felicia C. Mainella and Laura. Osteen. 2005. Developing a leadership identity: A grounded theory. Journal of College Student Development 46(6): 593-611.

Kotthoff, Helga. 2006. Pragmatics of performance and the analysis of conversational humor. Humor: International Journal of Humor Research 19(3): 271-304.

Krantz, James. 1990. Lessons from the field: An essay on the crisis of leadership in contemporary organizations. The Journal of Applied Behavioral Science 26(1): 49.

Kuiper, Koenraad and Michelle Lodge. 2004. Formulae of command: Form and function in controlling the body. In Csaba Földes (ed.), Res humanae proverbiorum et sententiarum: Ad honorem Wolfgangi Mieder, 141-153. Tübingen: Gunter Narr Verlag.

Kuiper, Koenraad. 1991. Sporting formulae in New Zealand English: Two models of male solidarity. In Jenny Cheshire (ed.), English around the world: Sociolinguistic perspectives, 200-212. Cambridge, U.K.: Cambridge University Press.

Kuiper, Koenraad. 1996. Smooth talkers: The linguistic performance of auctioneers and sportscasters, Mahwah, New Jersey: Lawrence Erlbaum.

Kuiper, Koenraad. 2009. Formulaic genres, Basingstoke, U.K.: Palgrave Macmillan.

Ladegaard, Hans J. 2011a. Stereotypes and the discursive accomplishment of intergroup differentiation: Talk about "the other" in a global business organization.

Pragmatics 21(1): 85-109. 
Ladegaard, Hans J. 2011b. "Doing power" at work: Responding to male and female management styles in a global business corporation. Journal of Pragmatics 43(1): 4-19.

Lakoff, Robin. 1973. Language and woman's place. Language in Society 2(1): 45-80.

Lave, Jean and Etienne Wenger. 1991. Situated learning: Legitimate peripheral participation, Cambridge, U.K.: Cambridge University Press.

Law, Robin. 1997. Masculinity, place, and beer advertising in New Zealand: The southern man campaign. New Zealand Geographer 53(2): 22-28.

Lazaraton, Anne. 2003. Evaluative criteria for qualitative research in applied linguistics: Whose criteria and whose research? The Modern Language Journal 87(1): 1-12.

Leech, Geoffrey. 1999. The distribution and function of vocatives in American and British English conversation. In, Out of corpora: Studies in honour of Stig Johansson, 107-118. Amsterdam, The Netherlands: Rodopi.

Lefebvre, Henri. 1991. The production of space, Wiley-Blackwell.

Levinson, Stephen. 1983. Pragmatics, Cambridge, U.K.: Cambridge University Press.

Light, Richard and David Kirk. 2000. High school rugby, the body and the reproduction of hegemonic masculinity. Sport, Education and Society 5(2): 163.

Limbrick, Peter. 1991. A study of male and female expletive use in single and mixed-sex situations. Te Reo (34): 71-89.

Ling, Chen. 2008. Leaders or leadership: Alternative approaches to leadership studies. Management Communication Quarterly 21(4): 547-555.

Locher, Miriam A. and Richard J. Watts. 2005. Politeness theory and relational work. Journal of Politeness Research. Language, Behaviour, Culture 1(1): 9.

Lord, Robert George and Douglas J. Brown. 2004. Leadership processes and follower selfidentity, Mahwah, New Jersey: Lawrence Erlbaum.

LWP. 2010. LWP - The Language in the Workplace Project. The Language in the Workplace Project. Available at: http://www.victoria.ac.nz/lals/lwp/ [Accessed July 6, 2011].

MacLean, Malcolm. 1999. Of warriors and blokes: The problem of Maori rugby for Pakeha masculinity in New Zealand. In Timothy John Lindsay Chandler and John Nauright (eds.), Making the rugby world: Race, gender, commerce, 1-26. Routledge.

MacLean, Malcolm. 2000. Football as social critique: Protest movements, rugby and history in Aotearoa, New Zealand. International Journal of the History of Sport 17(2): 255-277.

MacLean, Malcolm. 2003. Making strange the country and making strange the countryside: Spatialized clashes in the affective economies of Aotearoa/New Zealand during the 1981 Springbok rugby tour. In John Bale and Mike Cronin (eds.), Sport and postcolonialism, 57-72. Oxford: Berg.

MacLean, Malcolm. 2004. The routine rituals of run-of-the-mill rugby: Sport, identities and everyday life. Paper presented at Educational and Sociological Issues in Sports And Physical Recreation Conference, 8 December, 2004, at University of Århus. The routine rituals of run-of-the-mill rugby: Sport, identities and everyday life.

Malmberg, Torsten. 1980. Human territoriality: Survey of behavioural territories in man with preliminary analysis and discussion of meaning, Berlin: Mouton de Gruyter.

Mandell, Barbara and Shilpa Pherwani. 2003. Relationship between emotional intelligence and transformational leadership style: A gender comparison. Journal of Business and Psychology 17(3): 387-404. 
Marra, Meredith, Bernadette Vine and Janet Holmes. 2008. Heroes, fathers and good mates: Leadership styles of men at work. July, 2008, at Wellington. Power and Place: proceedings of ANZCA08.

Martin, Gerard John. 2008. The game is not the same: A history of professional rugby in New Zealand. MA Thesis. Auckland, New Zealand: AUT.

Masterson, Julie J, Lisa K Davies and Gerald L Masterson. 2006. Coach talk: Linguistic demands inherent in youth sports. Language 37(1): 39-49.

McConnell, Robin. 1998. Inside the All Blacks, Auckland, New Zealand: HarperCollins.

McConnell, Robin. 1996. Sport team leadership: Coaching and captaincy in elite level rugby union football. PhD Thesis. University of Waikato.

Meân, Lindsey J. and Kelby K. Halone. 2010. Sport, language, and culture: Issues and intersections. Journal of Language and Social Psychology 29(3): 253 -260.

Meân, Lindsey J. 2001. Identity and discursive practice: Doing gender on the football pitch. Discourse and Society 12(6): 789-815.

Melnick, Merrill J. and John W. Loy. 1996. The effects of formal structure on leadership recruitment: An analysis of team captaincy among New Zealand provincial rugby teams. International Review for the Sociology of Sport 31(1): 91-105.

Melnick, Merrill J. and Rex W Thomson. 1996. The Maori people and positional segregation in New Zealand rugby football. International Review for the Sociology of Sport 31(2): 139.

Messner, Michael A. 1995. Power at play: Sports and the problem of masculinity, Boston, Massachussetts: Beacon Press.

Meyerhoff, Miriam. 1994. Sounds pretty ethnic, eh?: A pragmatic particle in New Zealand English. Language in Society 23(3): 367-388.

Meyerhoff, Miriam. 2001. Communities of practice. In J. K. Chambers, Peter Trudgill, and Natalie Schilling-Estes (eds.), The handbook of language variation and change, 527-548. Malden, Massachussetts: Blackwell.

Meyerhoff, Miriam. 2005. Biographies, agency and power. Journal of Sociolinguistics 9(4): 595-601.

Milroy, Lesley. 1987. Language and social networks 2nd ed., Oxford, U.K: Blackwell.

Mirivel, Julien C. and Karen Tracy. 2005. Premeeting talk: An organizationally crucial form of talk. Research on Language \& Social Interaction 38(1): 1 - 34.

Mizruchi, Mark S. 1985. Local sports teams and celebration of community: A comparative analysis of the home advantage. The Sociological Quarterly 26(4): 507-518.

Mondada, Lorenza. 2009. Emergent focused interactions in public places: A systematic analysis of the multimodal achievement of a common interactional space. Journal of Pragmatics 41(10): 1977-1997.

Moore, Emma. 2006. "You tell all the stories": Using narrative to explore hierarchy within a Community of Practice. Journal of Sociolinguistics 10(5): 611-640.

Moore, Emma. 2010a. Communities of practice and peripherality. In Carmen Llamas and Dominic Watt (eds.), Language and identities, 123-133. Edinburgh, U.K.: Edinburgh University Press.

Moore, Emma. 2010b. Interaction between social category and social practice: Explaining was/were variation. Language Variation and Change 22(03): 347371.

Moore, James C and Jody Brylinsky. 1995. Facility familiarity and the home advantage. Journal of Sport Behavior 18(4). 
Morgan, Marcyliena. 2010. The presentation of indirectness and power in everyday life. Journal of Pragmatics 42(2): 283-291.

Morgeson, Frederick P., D. Scott DeRue and Elizabeth P. Karam. 2010. Leadership in teams: A functional approach to understanding leadership structures and processes. Journal of Management 36(1): 5-39.

Morris, Desmond. 1981. The soccer tribe, London: Cape.

Mullany, Louise. 2010. Gendered identities in the professional workplace: Negotiating the glass ceiling. In Carmen Llamas and Dominic Watt (eds.), Language and identities, 179-192. Edinburgh: Edinburgh University Press.

Mullany, Louise. 2007. Gendered discourse in the professional workplace, Basingstoke, U.K.; New York: Palgrave Macmillan.

Murata, Kazuyo. 2009. Laughter for defusing tension: Examples from business meetings in Japanese and in English. In Hiromitsu Hattori, Takahiro Kawamura, Tsuyoshi Idé, Makoto Yokoo, and Yohei Murakami (eds.), New Frontiers in Artificial Intelligence, 294-305. Berlin, Heidelberg: Springer.

Nauright, John and Timothy John Lindsay Chandler. (eds.) 1996. Making men: Rugby and masculine identity, London; Portland, Oregon: Frank Cass \& Co.

Nauright, John. 1990. Myth and reality: Reflections on rugby and New Zealand historiography. Sporting Traditions 6(2): 219-30.

Nauright, John. 1996. Sustaining masculine hegemony: Rugby and the nostalgia of masculinity. In John Nauright and Timothy John Lindsay Chandler (eds.), Making men: Rugby and masculine identity, 227-244. London: Frank Cass \& Co.

Nixon, Anne and Charmaine Power. 2007. Towards a framework for establishing rigour in a discourse analysis of midwifery professionalisation. Nursing Inquiry 14(1): 71-79.

Nykodym, Nick and John A. Boyd. 1975. Expletive deleted: A study of language usage. Paper presented at Annual Meeting of the International Communication Association, April, 1975, at Chicago. Expletive deleted: A study of language usage.

NZ Herald. 2010. State of rugby part 6: Club and amateur rugby - state of rugby - nz herald videos,

NZRU. 2007a. Coaching toolbox - development. Available at: http://www.coachingtoolbox.co.nz/toolbox [Accessed September 25, 2008].

NZRU. 2007b. National competitions. Available at: http://www.nzru.co.nz/History/NationalCompetitions/tabid/1191/Default.aspx [Accessed September 28, 2008].

NZRU. 2008. Air New Zealand Cup competitions review, Wellington, New Zealand: NZRU.

NZRU. 2009. 2009 annual report, Wellington, New Zealand: NZRU.

O’Toole, James, Jay Galbraith and Edward E. Lawler. 2002. When two (or more) heads are better than one: The promise and pitfalls of shared leadership. California Management Review 44(4): 65-83.

Obel, Camilla. 2001. Unions, leagues and franchises: The social organisation of rugby union in New Zealand. PhD Thesis. Christchurch, New Zealand: University of Canterbury.

Obel, Camilla. 2007. Celebration and marginalisation in New Zealand sport: The'ethnic' national Maori rugby union team. International Journal of Sport Management and Marketing 2(1): 146-159.

Ochs, Elinor. 1990. Indexicality and socialization. In James W Stigler, Richard A Shweder, and Gilbert H Herdt (eds.), Cultural psychology: Essays on comparative human development, 287-308. 
Ochs, Elinor. 1993. Constructing social identity: A language socialization perspective. Research on Language and Social Interaction 26(3): 287-306.

Oliver, Marion M. and Joan Rubin. 1975. The use of expletives by some American women. Anthropological Linguistics 17(5): 191-197.

Owen, P. Dorian and Clayton R. Weatherston. 2002. Professionalization of New Zealand rugby union: Historical background, structural changes and competitive balance. University of Otago Economics Discussion Papers (0214).

Owen-Pugh, Valerie. 2007. Theorizing sport as a community of practice: The coachathlete relationship in British professional basketball. In Jason Hughes, Nick Jewson, and Lorna Unwin (eds.), Communities of Practice: Critical perspectives, 83-95. London: Routledge.

Palmer, Farah Rangikoepa and Tina M. Masters. 2010. Maori feminism and sport leadership: Exploring Maori women's experiences. Sport Management Review 13(4): 331-344.

Palmer, Farah Rangikoepa. 2000. Maori girls, power, physical education, sport, and play: "Being hungus, hori, and hoha." Dunedin: University of Otago.

Paul, Gregor. 2007. Graham Henry's revolution. New Zealand Herald.

Paul, Gregor. 2008. Loss leaders. NZ Rugby World (114): 34-36.

Pearce, Craig L. and Jay Alden Conger. 2003. Shared leadership: Reframing the hows and whys of leadership, Sage.

Peeters, Bert. 2004. Tall poppies and egalitarianism in Australian discourse: From key word to cultural value. English World-Wide 25(1): 1-25.

Pennycook, Alastair. 2004. Performativity and language studies. Critical Inquiry in Language Studies 1(1): 1.

Pennycook, Alastair. 2010. Language as a local practice 1st ed., Abingdon, U.K.; New York: Routledge.

Phillips, Jock. 1996. The hard man: Rugby and the formation of male identity in New Zealand. In John Nauright and Timothy John Lindsay Chandler (eds.), Making men: Rugby and masculine identity, 70-90. London: Frank Cass \& Co.

Pollard, R. 2008. Home advantage in football: A current review of an unsolved puzzle. The Open Sports Sciences Journal 1: 12-14.

Pringle, Richard. 2001. Competing discourses: Narratives of a fragmented self, manliness and rugby union. International Review for the Sociology of Sport 36(4): 425-439.

Raelin, Joe. 2011. From leadership-as-practice to leaderful practice. Leadership 7(2): $195-211$.

Rampton, Ben. 2006. Language in late modernity: Interaction in an urban school, Cambridge, U.K.: Cambridge University Press.

Rampton, Ben. 2007a. Neo-Hymesian linguistic ethnography in the United Kingdom. Journal of Sociolinguistics 11(5): 584-607.

Rampton, Ben. 2007b. Linguistic ethnography, interactional sociolinguistics and the study of identities.

Rendle-Short, Johanna. 2009. The address term "mate" in Australian English: Is it still a masculine term? Australian Journal of Linguistics 29(2): 245-268.

Ribeiro, Branca Telles. 2006. Footing, positioning, voice. Are we talking about the same things? In Anna De Fina, Deborah Schiffrin, and Michael Bamberg (eds.), Discourse and identity, 48. Cambridge, U.K.: Cambridge University Press.

Richards, Keith. 2006. Language and professional identity: Aspects of collaborative interaction, Basingstoke, U.K.: Palgrave Macmillan. 
Richards, Keith. 2010. Professional orientation in back region humor. Text \& Talk-An Interdisciplinary Journal of Language, Discourse \& Communication Studies 30(2): 145-167.

Rigauer, Bero. 1981. Sport and work, New York: Columbia University Press.

Rock, Frances. 2005. "I've picked some up from a colleague": Language, sharing and communities ofpractice in an institutional setting. In David Barton and Karin Tusting (eds.), Beyond communities of practice: Language, power, and social context, 77-104. Cambridge, U.K.: Cambridge University Press.

Rost, Joseph Clarence. 1993. Leadership for the twenty-first century, Westport, Connecticut: Praeger.

Ryan, Greg. 1993. Forerunners of the All Blacks: The 1888-89 New Zealand Native Football Team in Britain, Australia and New Zealand, Christchurch, New Zealand: Canterbury University Press.

Ryan, Greg. 2005a. The end of an aura: All Black rugby and rural nostalgia in the professional era. In Greg Ryan (ed.), Tackling rugby myths: Rugby and New Zealand society, 1854-2004, 151-172. Dunedin, New Zealand: University of Otago Press.

Ryan, Greg. 2005b. Rural myth and urban actuality: The anatomy of All Black and New Zealand rugby 1884-1938. In Greg Ryan (ed.), Tackling rugby myths: Rugby and New Zealand society, 1854-2004, 33-54. Dunedin, New Zealand: University of Otago Press.

Saito, Junko. 2009. Another look at directive usage by people of authority: The case of Japanese male superiors in a workplace. Sophia Linguistica 57: 319-341.

Sally, David. 2002. Co-leadership: Lessons from republican Rome. California Management Review 44(4): 84-99.

Sansone, David. 1988. Greek athletics and the genesis of sport, Berkeley, California: University of California Press.

Sarangi, Srikant and Christopher N. Candlin. 2003. Trading between reflexivity and relevance: New challenges for applied linguistics. Applied Linguistics 24(3): 271 285.

Sarangi, Srikant and Celia Roberts. 1999. The dynamics of interactional and institutional orders in work-related settings. In Srikant Sarangi and Celia Roberts (eds.), Talk, work and institutional order: Discourse in medical, mediation and management settings, 1-57. Berlin: Mouton de Gruyter.

Sarangi, Srikant. 2004. Language/activity: Observing and interpreting ritualistic institutional discourse. Cahiers de Linguistique Française 26(special issue on "Les modèles du discours face au concept d'action".): 136-150.

Sarangi, Srikant. 2005. The conditions and consequences of professional discourse studies. Journal of Applied Linguistics 2(3): 371-394.

Sarangi, Srikant. 2007. Editorial: The anatomy of interpretation: Coming to terms with the analyst's paradox in professional discourse studies. Text \& Talk 27(5/6): 567-584.

Schaaf, Matani. 2006. Elite pacific male rugby players' perceptions and experiences of professional rugby. Junctures: The Journal for Thematic Dialogue (7): 41(14).

Schacht, Steven P. 1996. Misogyny on and off the "pitch": The gendered world of male rugby players. Gender and Society 10(5): 550-565.

Schegloff, Emanuel A. 2000. Overlapping talk and the organization of turn-taking for conversation. Language in Society 29(1): 1-63. 
Schensul, Stephen L., Jean J. Schensul and Margaret Diane LeCompte. 1999. Essential ethnographic methods: Observations, interviews, and questionnaires, Walnut Creek, California: Rowman Altamira.

Schiffrin, Deborah. 1987. Discourse markers, Cambridge, U.K.: Cambridge University Press.

Schiffrin, Deborah. 2009. Crossing boundaries: The nexus of time, space, person, and place in narrative. Language in Society 38(04): 421-445.

Schlager, M. S. and J. Fusco. 2004. Teacher professional development, technology, and communities of practice. In Sasha A. Barab, Rob Kling, and James H. Gray (eds.), Designing for virtual communities in the service of learning, 120-153. Cambridge, U.K.: Cambridge University Press.

Schneider, Edgar W. 2008. Accommodation versus identity? A response toTrudgill. Language in Society 37(2): 262-266.

Schnurr, Stephanie and Angela Chan. 2009. Politeness and leadership discourse in New Zealand and Hong Kong: A cross-cultural case study of workplace talk. Journal of Politeness Research 5(2): 131-157.

Schnurr, Stephanie, Meredith Marra and Janet Holmes. 2007. Being (im)polite in New Zealand workplaces: Maori and Pakeha leaders. Journal of Pragmatics 39(4): 712-729.

Schnurr, Stephanie. 2008a. Constructing leader identities through teasing at work. Journal of Pragmatics 41(6): 1125-1138.

Schnurr, Stephanie. 2008b. Leadership discourse at work: Interactions of humour, gender and workplace culture, New York: Palgrave Macmillan.

Scollon, Ron and Suzie Wong Scollon. 2007. Nexus analysis: Refocusing ethnography on action. Journal of Sociolinguistics 11(5): 608-625.

Sheard, Kenneth G. and Eric G. Dunning. 1973. The rugby football club as a type of "male preserve": Some sociological notes. International Review for the Sociology of Sport 8(3): 5-24.

Silverman, D. 2006. Interpreting qualitative data 3rd ed., London: Sage.

Silverstein, Michael. 1992. The uses and utility of ideology: Some reflections. Pragmatics 2(3): 311-323.

Silverstein, Michael. 2003. Indexical order and the dialectics of sociolinguistic life. Language \& Communication 23(3-4): 193-229.

Simonsen, Kirsten. 2007. Practice, spatiality and embodied emotions: An outline of a geography of practice. Human Affairs 17(2): 168-181.

Smith, Jonathan Z. 1980. The bare facts of ritual. History of Religions 20(1/2): 112-127.

Sobociński, Mikołaj. 2010. On proxemics and territoriality in communicative behavior of man - A communiqué. 15 May, 2010, at Wrocław, Poland. Consultant assembly III: In search of innovatory subjects for language and culture courses.

Sommer, Robert. 1961. Leadership and group geography. Sociometry 24(1): 99-110.

Spencer-Oatey, Helen. 2005. (Im)politeness, face and perceptions of rapport: Unpackaging their bases and interrelationships. Journal of Politeness Research. Language, Behaviour, Culture 1(1): 95.

Staley, Constance M. 1978. Male-female use of expletives: A heck of a difference in expectations. Anthropological Linguistics 20(8): 367-380.

Stapleton, Karyn. 2003. Gender and swearing: A community practice. Women and Language 26(2): 22-34.

Stubbe, Maria and Janet Holmes. 1995. You know, eh and other "exasperating expressions": An analysis of social and stylistic variation in the use of pragmatic 
devices in a sample of New Zealand English. Language and Communication 15(1): 63-88.

Stubbe, Maria and Janet Holmes. 2000. Talking Maori or Pakeha in English: Signalling identity in discourse. In Allan Bell and Koenraad Kuiper (eds.), New Zealand English, 249-278. Wellington, New Zealand: Victoria University Press.

Sullivan, Philip J. and Deborah L. Feltz. 2003. The preliminary development of the Scale for Effective Communication in Team Sports (SECTS). Journal of Applied Social Psychology 33(8): 1693-1715.

Svennevig, Jan. 2008. Exploring leadership conversations. Management Communication Quarterly 21(4): 529-536.

Tagg, Brendon. 2008. Imagine, a man playing netball!': Masculinities and sport in New Zealand. International Review for the Sociology of Sport 43(4): 409-430.

Takano, Shoji. 2005. Re-examining linguistic power: Strategic uses of directives by professional Japanese women in positions of authority and leadership. Journal of Pragmatics 37(5): 633-666.

Tannen, Deborah. 1986. Introducing constructed dialogue in Greek and American conversational and literary narrative. In Florian Coulmas (ed.), Direct and indirect speech, 11-32. Berlin; New York: Mouton De Gruyter.

Tannen, Deborah. 1990. Gender differences in topical coherence: Creating involvement in best friends' talk. Discourse Processes 13(1): 73-90.

Tannen, Deborah. 2001. You just don't understand: Women and men in conversation, Harper Paperbacks.

Tannen, Deborah. 2005. Interactional sociolinguistics as a resource for intercultural pragmatics. Intercultural Pragmatics 2(2): 205-208.

Telfer, Jim and David Ferguson. 2006. Jim Telfer, Mainstream Publishing.

Terret, Thierry. 1999. Learning to be a man: French rugby and masculinity. In Timothy John Lindsay Chandler and John Nauright (eds.), Making the rugby world: Race, gender, commerce, 63-87. London; New York: Routledge.

Thompson, Edward H and Patrick M Whearty. 2004. Older men's social participation: The importance of masculinity ideology. The Journal of Men's Studies 13(1): 5-24.

Thompson, Shona M. 1988. Challenging the hegemony: New Zealand women's opposition to rugby and the reproduction of a capitalist patriarchy. International Review for the Sociology of Sport 23(3): 205 -212.

Tonts, Matthew and Kim Atherley. 2010. Competitive sport and the construction of place identity in rural Australia. Sport in Society 13(3): 381-398.

Trester, Anne Marie. 2009. Discourse marker "oh" as a means for realizing the identity potential of constructed dialogue in interaction. Journal of Sociolinguistics 13(2): 147-168.

Turman, Paul D. 2003. Coaches and cohesion: The impact of coaching techniques on team cohesion in the small group sport setting. Journal of Sport Behavior 26(1): 86-105.

Tusting, Karin. 2005. Language and power in communities of practice. In David Barton and Karin Tusting (eds.), Beyond communities of practice: Language, power, and social context, 36-54. Cambridge, U.K.: Cambridge University Press.

Uhl-Bien, Mary. 2006. Relational leadership theory: Exploring the social processes of leadership and organizing. The Leadership Quarterly 17(6): 654-676.

Vaughan, Elaine. 2007. "I think we should just accept ... our horrible lowly status": Analysing teacher-teacher talk within the context of community of practice. Language Awareness 16(3): 173-189. 
Vine, Bernadette, Janet Holmes, Meredith Marra, Dale Pfeifer and Brad Jackson. 2008. Exploring co-leadership talk through interactional sociolinguistics. Leadership 4(3): 339-360.

Vine, Bernadette. 2004. Getting things done at work: The discourse of power in workplace interaction, Philadelphia, Pennsylvania: John Benjamins.

Vroom, Victor H and Arthur G Jago. 2007. The role of the situation in leadership. American Psychologist 62(1): 17.

Waitzkin, Howard. 1990. On studying the discourse of medical encounters: A critique of quantitative and qualitative methods and a proposal for reasonable compromise. Medical Care 28(6): 473-488.

Walsh, David. 2004. Doing ethnography. In Clive Seale (ed.), Researching society and culture, 225-237. London; Thousand Oaks, California: Sage.

Watson, Geoff. 2007. Sport and ethnicity in New Zealand. History Compass 5(3): 780801.

Watts, Richard J. 2003. Politeness, Cambridge, U.K.: Cambridge University Press.

Wellard, Ian. 2002. Men, sport, body performance and the maintenance of 'exclusive masculinity'. Leisure Studies 21(3): 235-247.

Wenger, Etienne. 1998a. Communities of practice: Learning, meaning, and identity, Cambridge, U.K.: Cambridge University Press.

Wenger, Etienne. 1998b. Communities of practice: Learning as a social system. Systems thinker 9(5): 1-5.

Werlen, Benno. 1993. Society action and space: An alternative human geography, London: Routledge.

Wieczorek, Anna Ewa. 2009. This is to say you're either in or out: Some remarks on clusivity. Critical approaches to discourse analysis across disciplines 3(2): 118129.

Wilson, Nick. forthcoming. The portable team-room: Discursive constructions of rugby team identity at home and away. In Brian W. King, Jeannie Fletcher, and Martin Paviour-Smith (eds.), Language, space and place: Performing sites of social interaction. Oxford, U.K.: Oxford University Press.

Wilson, Nick. 2007. Leading through language: An analysis of the relational practice of male leaders through ethnographic engagement. Masters Thesis. Edinburgh: University of Edinburgh.

Wilson, Nick. 2009a. The discourse of deputies: Communicating co-leadership in a rugby club. Te Reo 52: 73-98.

Wilson, Nick. 2009b. "Fucking" as a solidarity marker. Paper presented at The Linguistic Society of New Zealand 18th Biennial Conference, 1 December, 2009, at Massey University, Palmerston North, New Zealand.

Wilson, Nick. 2010. Bros, boys and guys: Address term function and communities of practice in a New Zealand rugby team. New Zealand English Journal 24: 33-54.

Winston, Bruce E and Kathleen Patterson. 2006. An integrative definition of leadership. International Journal of Leadership Studies 1(2): 6-66.

Wolcott, Harry F. 2002. Writing up qualitative research... better. Qualitative Health Research 12(1): 91-103.

Wolfson, Nessa. 1976. Speech events and natural speech: Some implications for sociolinguistic methodology. Language in Society 5(02): 189-209.

Wray, Alison and Michael R. Perkins. 2000. The functions of formulaic language: An integrated model. Language \& Communication 20(1): 1-28. 


\section{N. A. Wilson}

Zhang, Jianhui and Barbara E. Jensen. 1997. Modification and revision of the leadership scale for sport. Journal of Sport Behavior 20(1): 105.

Zinn, Caryn. 2004. Nutrition knowledge of New Zealand premier club rugby coaches. Masters Thesis. Auckland, New Zealand: AUT. 


\section{Appendix A: Glossary of Rugby Terms}

This is not an exhaustive description of the game of rugby. However, it provides illumination of a number of key terms that are essential for the understanding of the activities that the Prems train for. For a basic description of the game of rugby, I would recommend the following websites:

http://guide.rugbyrugby.com/Rugby\%20Sections/Beginners\%20Guide/Basic\%20Rule s\%20Updated.asp

http://news.bbc.co.uk/sport2/hi/rugby union/rules and equipment/4200680.stm

I, like many followers of rugby, gained my knowledge of the game from playing it and the explanation of rugby terminology here is drawn from my own knowledge of it as an insider. Of greater importance than the game-play related language is perhaps the language that forms the basis of a shared rugby player repertoire, as shown in the section on team specific language, gathered through the ethnography of the Prems.

In this glossary reference is made to attacking team and defending team. The attacking team is always the team that has possession of the ball while the defending team does not have the ball.

\subsection{Game-play}

\section{The rugby pitch}

\section{Standard Rugby Union pitch}

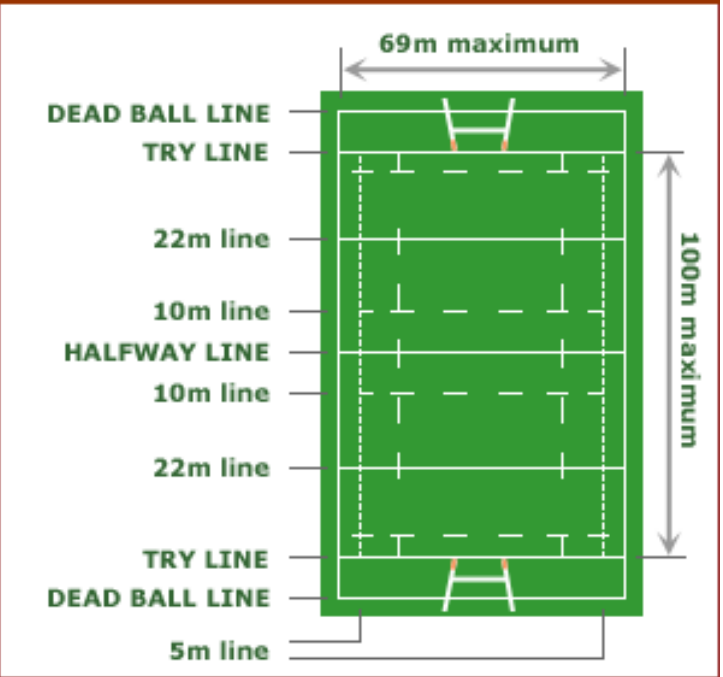

As can be seen from the image of the pitch, the rugby pitch is split into many different zones, and is crossed by many lines, each of which has a name in common rugby usage. These are labelled on the diagram. The most commonly referred to are probably the try line, the twenty-two and halfway. The other lines are used only in some situations. In addition to their role in the match, in which the lines 
represent markers for various set-pieces (i.e. a re-start situation such as a scrum or lineout), in training sessions they are often used in communicating instructions or directions. For instance, in Example 5.4 the players are instructed to stand "the other side of the line". In this case, the line being referred to was one of the try lines, and it happened to be the nearest line to where the training session was being conducted at this point. Thus the lines on the pitch feature as a vital deictic reference used in team discourse and knowledge of the name of each is a vital piece of socio-cultural knowledge for any rugby player (or coach).

\section{Scrum}

When the ball is knocked-on by a player, a scrum is awarded to the defending team. A scrum is when the forwards bind together in a set formation and interlock (engage) with the opposition, and the half-back of the team

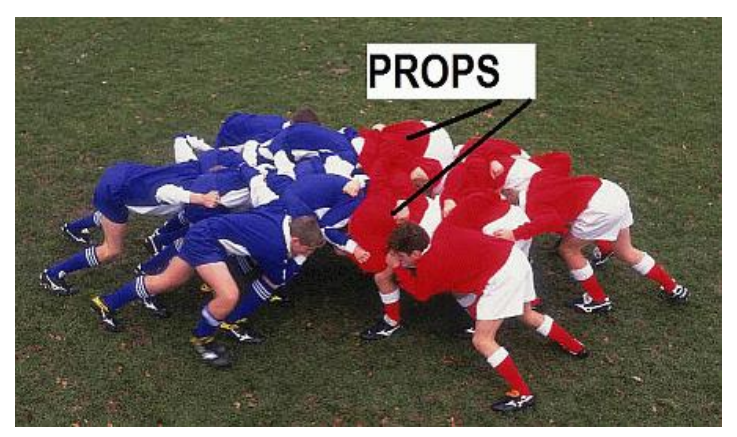
who was awarded the scrum rolls the ball in from the side of the scrum. Both hookers are then allowed to swing their feet (hook) for the ball as it rolls in order to push it back to their side and emerge from the back of the scrum where the half-back should pick it up. From this point on the ball is considered to be in open-play.

\section{Lineout}

When the ball goes out of play the defending team are awarded a lineout, unless the ball

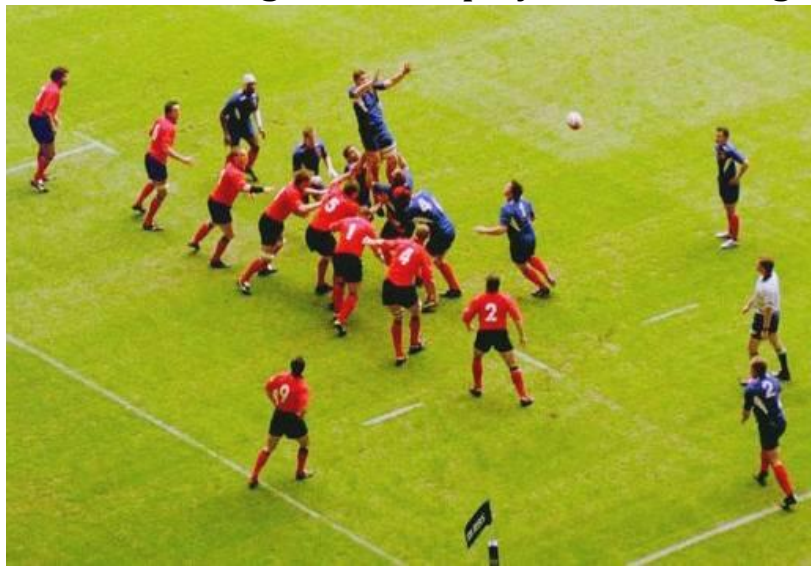
was kicked out of play from a penalty, in which case the team who were awarded the penalty kick get the lineout. In addition, if the kicker is standing in front of his team's twenty-two metre line when he kicks the ball, and it does not bounce before going into touch, then the lineout will be taken from where the ball was kicked from. Otherwise it would be taken from where the ball crossed the touch line. The lineout is taken when the attacking team's hooker throws in the ball, with the aim of one of the players in the line to jump (with assistance) and catch it. However, the 
opposition can also jump and attempt to intercept the throw. The throw must be straight down the middle of the line, so it may seem that each team has an equal opportunity to catch the ball. This is countered by the attacking team "calling the lineout". The hooker shouts a pre-arranged code, which signals who the ball is going to, whether they should catch it or pat it down to the half-back, and whether the catch will then turn into a driving maul or be immediately passed out to the backs.

\section{Rucks and mauls}

When the ball is in open play, and a player is tackled and falls to the ground, he must place the ball backwards, relinquishing contact with it (although the allowed time for him to do so varies). The first player to arrive at the tackle situation may then either pick up the ball (a risky proposition), or step over the tacklee and ball (and often the tackler), forming a ruck. Subsequent players in his team may not pick up the ball but must either join the ruck from behind the hindmost foot on their side of the ruck, or wait until the ruck gets over the ball, it may then be picked up. A player who has joined the ruck may not touch the ball with his hands. The other team contests the ruck by pushing against the rucking players and may push them off the ball, resulting in what is known as a turn-over.

If on the other hand a tackled player does not fall to the ground, but is supported by his team-mates, he forms the nucleus of a maul, in which the ball may be handled while each team (usually the forwards) tries to push the maul in the direction in which they are attacking and simultaneously gain control of the ball. If the team in possession does not manage a sustained movement then the whistle is blown and a scrum awarded to the team without the ball. A driving maul is the description for a pre-planned, organised maul in which the attacking team shields the ball from the defenders via a wellpractised routine of players successively binding onto the ball carrier, each smuggling the ball backwards, while moving the whole maul forwards.

Both the ruck and the maul are perhaps the areas of rugby that are most difficult for rugby outsiders to understand due to the many bodies involved and the number of possible outcomes. However, they can most simply be explained as a means for teams to contest possession of the ball following a tackle. 


\subsection{Training apparatus}

\section{Scrum machine}

A scrum machine is a piece of training apparatus used to practice scrums. Pictured here with and without players (these are not images from the Prems; they were found using Google Images). The images below are the closest to the basic type of scrum machine
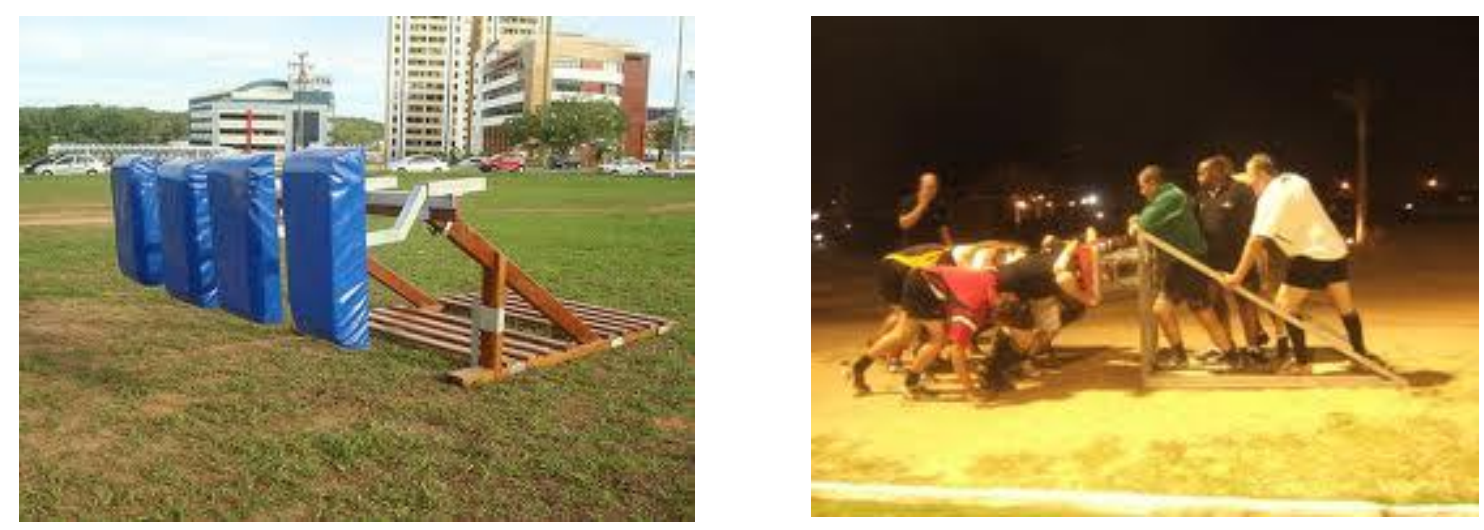

used by the Prems, although far more complex machines, with adjustments, wheels and weights are available. As in the picture featuring players, the weight was added to the machine using resting players (and me) as ballast. This was one way in which I was able to participate in training sessions. The object of scrum training was for the forwards to push the scrum machine a prescribed distance using the correct scrumagging technique of a concerted push. This was practiced with and without the ball being rolled in.

\subsection{General rugby vocabulary}

This only includes words or phrases used in the thesis, there are many more items in the repertoire of rugby language.

\section{Breakdown}

The situation following a tackle before a ruck, maul or pick-up has taken place.

\section{Contact}

The act of hitting another player with your body while running with the ball, i.e. being tackled, "taking the ball into contact".

\section{Drive}

Push forwards. Can be a verb or noun. E.g. "drive them back" or "get a good drive going in the scrum". 


\section{Drop kick}

A kick where the ball is dropped (on purpose) from the hands to bounce on the ground at the same time as it is kicked. This has to be done in order to score a drop goal. If the ball is kicked "from hand", then a goal cannot be scored, although this is typically how players kick for territory. A drop kick is also used as a means of restarting the game from the halfway line following points being scored.

\section{Number ones}

Smart clothes to be worn after a match. In the Prems this included smart shoes, trousers and a club shirt and tie. Players who had already played one season for the club also wore a club blazer. Apart from the blazer, this matches with my rugby experience. "Number Ones" is a term used, as far as I know, throughout the English speaking rugby world.

\subsection{Team specific language}

Every rugby team coins its own code words that are used for pre-planned moves. For reasons of confidentiality, a part of my ethical obligation to the team, these are not reproduced in this thesis. Other words have been substituted in their place, such as "pies" and "chips" being used as a way of calling a move from the breakdown. There were also code words relating to specific running moves, while the forwards used a combination of numbers, letters and words to call moves in the lineouts.

The "brothers" call used to mark the end of huddles has been discussed at several points in the body of this thesis, and so no further discussion is necessary other than to say that another formula used to close huddles is to do what Mozza did in Example 7.4 and shout the team name rather than "brothers". 


\section{Appendix B: List of participants}

All names used in this thesis are pseudonyms, in order to protect the identities of the participants. However, the lists below link these pseudonyms to the role/position that each played in the Prems. As the thesis focuses on leadership, this list follows the hierarchical structure of the team, incorporating each member's positional affiliation, and where appropriate, their position.

If the players have no official place in the hierarchy I have given an indication of their integration into their positional CofP.

The first table provides details of all the speakers who feature in the extracts, while the second table shows those who formed a part of the Prems but did not appear in these transcripts. It may be noted that all of the core members of the CofPs are in the first of these tables.

\begin{tabular}{|l|l|l|l|}
\hline Name & Hierarchical Position & Positional Affiliation & Playing Position \\
\hline Tommo & Head Coach & Forwards & Number Eight \\
\hline Parky & Assistant Coach & Backs & Centre \\
\hline Duncan & Squad Manager & Backs & unknown \\
\hline Jon & Captain & Forwards & Hooker/Prop \\
\hline Mozza & Co-captain & Forwards & Flanker \\
\hline Bug & Vice-captain & Backs & $\begin{array}{l}\text { Second Five- } \\
\text { eights/Centre }\end{array}$ \\
\hline Tommy & Core & & Number Eight \\
\hline Mason & Core & Forwards & Lock \\
\hline Ata & Core & Forwards & Prop \\
\hline Nika & Core & Forwards & Prop \\
\hline Will & Core & Forwards & Half-back \\
\hline Tui & Core & Backs & First five-eighths \\
\hline Rik & Peripheral (new but on & Backs & Winger \\
\hline Steve & inward trajectory) & & Lock \\
\hline Colin & Core & Forwards & Centre \\
\hline
\end{tabular}




\section{N. A. Wilson}

\begin{tabular}{|c|c|c|c|}
\hline & injury) & & \\
\hline Smithy & $\begin{array}{l}\text { Peripheral (moved frequently } \\
\text { between Prems and S1s) }\end{array}$ & Backs & Half-back \\
\hline Sean & $\begin{array}{l}\text { Peripheral (moved frequently } \\
\text { between Prems and S1s) }\end{array}$ & Backs & Full-back \\
\hline Rory & $\begin{array}{l}\text { Marginal (new move from S1s } \\
\text { mid-season) }\end{array}$ & Backs & $\begin{array}{l}\text { Centre/second } \\
\text { five-eighths }\end{array}$ \\
\hline Cliff & $\begin{array}{l}\text { Peripheral (moved frequently } \\
\text { between Prems and S1s) }\end{array}$ & Forwards & Lock \\
\hline Jeff C & $\begin{array}{l}\text { Peripheral (new on inward } \\
\text { trajectory) }\end{array}$ & Forwards & Flanker \\
\hline Jeff $\mathrm{H}$ & Core & Forwards & Flanker \\
\hline Giles & $\begin{array}{l}\text { Peripheral (new on inward } \\
\text { trajectory) }\end{array}$ & Forwards & Prop \\
\hline
\end{tabular}

\section{Members of the Prems not featured in the extracts}

\begin{tabular}{|l|l|l|l|}
\hline James & Squad Manager & Forwards & unknown \\
\hline Evan & Peripheral & Forwards & Hooker \\
\hline Mike & $\begin{array}{l}\text { Marginal (pro player involved } \\
\text { for two matches) }\end{array}$ & Forwards & Prop \\
\hline Rawiri & $\begin{array}{l}\text { Peripheral (although core } \\
\text { from previous season) }\end{array}$ & Forwards & Prop \\
\hline Ryan & $\begin{array}{l}\text { Peripheral (long term injury, } \\
\text { acted as manager's assistant) }\end{array}$ & Backs & unknown \\
\hline Cam & Peripheral & Forwards & Flanker \\
\hline Afi & $\begin{array}{l}\text { Peripheral (first season and } \\
\text { non-native speaker) }\end{array}$ & Backs & Winger \\
\hline Tia & $\begin{array}{l}\text { Peripheral (first season and } \\
\text { non-native speaker) }\end{array}$ & Backs & \begin{tabular}{l} 
Winger \\
\hline Ara
\end{tabular} \\
$\begin{array}{l}\text { Peripheral (first season and } \\
\text { non-native speaker) }\end{array}$ & $\begin{array}{l}\text { Started as core, then moved to } \\
\text { peripheral through severe } \\
\text { injury }\end{array}$ & Backs & Full-back \\
\hline Doddie & Backs & & \\
\hline
\end{tabular}


Appendix B: List of participants

\begin{tabular}{|l|l|l|l|}
\hline Callum & $\begin{array}{l}\text { Peripheral (moved up from } \\
\text { Colts late in the season and } \\
\text { stayed) }\end{array}$ & Backs & Full-back \\
\hline Ian & $\begin{array}{l}\text { Marginal (played only two } \\
\text { games, promoted from Colts) }\end{array}$ & Backs & First-five eighths \\
\hline Stu & $\begin{array}{l}\text { Marginal (promoted from } \\
\text { Colts midway through season, } \\
\text { but subsequently returned) }\end{array}$ & Backs & Full-back \\
\hline Blackie & Team-Physio & unknown & unknown \\
\hline
\end{tabular}

Finally, there were also members of coaching staff from the other teams in the club that featured on recordings, often through their interactions with Tommo and Parky.

\begin{tabular}{|l|l|}
\hline Johann & Player Development Officer \\
\hline Andrew & Colts Head Coach \\
\hline Norman & Colts Manager \\
\hline AJ & Colts Manager \\
\hline Justin Peters & Senior Firsts Coach \\
\hline
\end{tabular}




\section{Appendix C: List of Recorded Data}

\begin{tabular}{|c|c|c|c|c|}
\hline Date Recorded & Situation & Mic Location & Duration & Transcribed? \\
\hline $05 / 03 / 2009$ & Interview & NW & 0:04:08 & yes \\
\hline $10 / 03 / 2009$ & Interview & NW & 0:01:14 & yes \\
\hline $10 / 03 / 2009$ & Interview & NW & 0:10:12 & yes \\
\hline $24 / 03 / 2009$ & Interview & NW & $0: 05: 32$ & yes \\
\hline $26 / 03 / 2009$ & Interview & NW & 0:04:09 & yes \\
\hline $31 / 03 / 2009$ & Interview & NW & $0: 02: 21$ & yes \\
\hline $31 / 03 / 2009$ & Interview & NW & 0:01:49 & yes \\
\hline $02 / 04 / 2009$ & Interview & NW & 0:01:46 & yes \\
\hline 02/04/2009 & Interview & NW & 0:03:27 & yes \\
\hline 04/04/2009 & Full Matchday & Parky & $2: 49: 30$ & no \\
\hline 04/04/2009 & Full Matchday & Tommo & 0:15:05 & no \\
\hline 04/04/2009 & Full Matchday & Tommo & 0:02:00 & no \\
\hline 04/04/2009 & Full Matchday & Tommo & $2: 17: 14$ & no \\
\hline 07/04/2009 & Interview & NW & 0:03:36 & no \\
\hline $14 / 04 / 2009$ & Interview & NW & 0:02:31 & no \\
\hline $14 / 04 / 2009$ & Training & Jon & 1:43:02 & no \\
\hline $18 / 04 / 2009$ & Post-match Team Room & Team Room & $0: 12: 23$ & yes \\
\hline $18 / 04 / 2009$ & Pre-match Huddle & Team Room & 0:06:48 & yes \\
\hline $18 / 04 / 2009$ & Team Meeting & Team Room & $0: 13: 18$ & yes \\
\hline $18 / 04 / 2009$ & Pre-match Huddle & Team Room & 0:01:27 & yes \\
\hline $18 / 04 / 2009$ & Full-time Huddle & NW & 0:08:42 & yes \\
\hline $18 / 04 / 2009$ & Half-time Huddle & NW & 0:04:17 & yes \\
\hline $18 / 04 / 2009$ & Warm-up & NW & $0: 17: 35$ & yes \\
\hline $21 / 04 / 2009$ & Training & Tommo & $1: 21: 26$ & no \\
\hline $21 / 04 / 2009$ & Training & Parky & 1:38:06 & no \\
\hline $21 / 04 / 2009$ & Training & Bug & $1: 51: 13$ & no \\
\hline $21 / 04 / 2009$ & Training & Jon & $1: 51: 37$ & no \\
\hline $23 / 04 / 2009$ & Training & Tommo & 1:47:56 & no \\
\hline $23 / 04 / 2009$ & Training & Duncan & 1:49:26 & no \\
\hline $23 / 04 / 2009$ & Training & Mozza & 1:49:04 & no \\
\hline $23 / 04 / 2009$ & Training & Afi & $1: 48: 38$ & no \\
\hline $25 / 04 / 2009$ & Half-time Huddle & NW & 0:06:04 & yes \\
\hline $25 / 04 / 2009$ & Full-time Huddle & NW & 0:01:44 & yes \\
\hline $25 / 04 / 2009$ & Pre-match Huddle & Team Room, stationary & 0:03:47 & no \\
\hline $25 / 04 / 2009$ & Pre-match Huddle & Team room, NW & 0:02:47 & yes \\
\hline $25 / 04 / 2009$ & Team Meeting & Team Room, stationary & $0: 12: 54$ & yes \\
\hline $25 / 04 / 2009$ & Warm-up & NW & 0:09:22 & no \\
\hline $28 / 04 / 2009$ & Training & Tommo & 1:33:08 & some \\
\hline $28 / 04 / 2009$ & Training & Tommo & 0:06:15 & some \\
\hline 28/04/2009 & Training & Parky & $1: 24: 34$ & some \\
\hline 28/04/2009 & Training & Mason & 1:37:09 & some \\
\hline
\end{tabular}




\section{N. A. Wilson}

\begin{tabular}{|c|c|c|c|c|}
\hline $28 / 04 / 2009$ & Training & Smithy & $1: 37: 00$ & some \\
\hline $2 / 05 / 2009$ & Half-time Huddle & NW & $0: 05: 22$ & yes \\
\hline $2 / 05 / 2009$ & Full-time Huddle & NW & $0: 02: 47$ & yes \\
\hline $2 / 05 / 2009$ & Full-time Huddle & NW & 0:00:58 & yes \\
\hline $2 / 05 / 2009$ & Warm-up & Team Room, stationary & $0: 15: 51$ & no \\
\hline $2 / 05 / 2009$ & Pre-match Huddle & Team Room, stationary & $0: 06: 29$ & yes \\
\hline $2 / 05 / 2009$ & Warm-up & NW & $0: 01: 21$ & no \\
\hline $2 / 05 / 2009$ & Warm-up & NW & 0:01:04 & no \\
\hline $2 / 05 / 2009$ & Warm-up & NW & 0:00:49 & no \\
\hline $2 / 05 / 2009$ & Warm-up & NW & $0: 00: 28$ & no \\
\hline $6 / 06 / 2009$ & Half-time Huddle & NW & $0: 05: 18$ & yes \\
\hline $6 / 06 / 2009$ & Team Meeting & Team Room, stationary & $0: 12: 05$ & yes \\
\hline $6 / 06 / 2009$ & Full-time Huddle & NW & 0:01:05 & yes \\
\hline $6 / 06 / 2009$ & Front Row Warm-up & NW & $0: 10: 35$ & no \\
\hline $6 / 06 / 2009$ & Full-time Huddle & NW & $0: 02: 50$ & yes \\
\hline $6 / 06 / 2009$ & Pre-warmup Huddle & NW & $0: 00: 24$ & yes \\
\hline $6 / 06 / 2009$ & Pre-match Huddle & Team Room, stationary & 0:04:06 & yes \\
\hline $6 / 06 / 2009$ & Pre-match Huddle & Team Room, stationary & 0:03:03 & yes \\
\hline $25 / 07 / 2009$ & Forwards Huddle & Team Room, stationary & $0: 01: 40$ & yes \\
\hline $25 / 07 / 2009$ & Full-time Huddle & NW & $0: 04: 23$ & yes \\
\hline $25 / 07 / 2009$ & Half-time Huddle & NW & 0:04:19 & yes \\
\hline $25 / 07 / 2009$ & Pre-match Huddle & Team Room, stationary & 0:03:08 & yes \\
\hline $25 / 07 / 2009$ & Pre-warmup locker room & Team Room, stationary & $0: 21: 47$ & no \\
\hline $25 / 07 / 2009$ & Team Meeting & Team Room, stationary & 0:19:00 & yes \\
\hline $25 / 07 / 2009$ & Warm-up & NW & $0: 01: 26$ & no \\
\hline $25 / 07 / 2009$ & Warm-up Huddle & NW & $0: 01: 47$ & no \\
\hline $25 / 07 / 2009$ & Water Message & Bug & 0:03:19 & yes \\
\hline
\end{tabular}

Total Recorded Duration 31:59:40

\begin{tabular}{lr}
\hline Total Interviews & $0: 40: 45$ \\
Total Training & $21: 58: 34$ \\
Total Match & $9: 20: 21$ \\
\hline
\end{tabular}




\section{Appendix D: Ethics Approval}

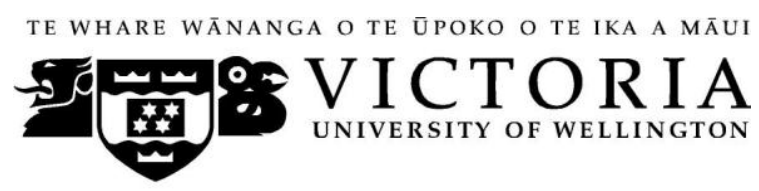

\section{MEMORANDUM}

Phone 0-4-463 5676

Fax 0-4-463 5209

\begin{tabular}{l|l}
\hline TO & Nick Wilson \\
\hline COPY TO & Professor Janet Holmes, Dr Meredith Marra, Supervisors \\
\hline FROM & Dr Allison Kirkman, Convener, Human Ethics Committee \\
\hline
\end{tabular}

\begin{tabular}{l|l}
\hline DATE & December 17, 2008 \\
\hline PAGES & 1 \\
\hline
\end{tabular}

SUBJECT $\quad$ Ethics Approval: No 16248, Playing with Language: the discursive construction of rugby as a workplace.

Thank you for your application for ethical approval, which has now been considered by the Standing Committee of the Human Ethics Committee.

Your application has been approved from the above date and this approval continues until 30 September 2011. If your data collection is not completed by this date you should apply to the Human Ethics Committee for an extension to this approval.

Best wishes with the research.

\section{Allison Kirkman}

Convener 


\section{N. A. Wilson}

TE WHARE WÁNANGa O TE OPOKO O TE IKA A MÁU

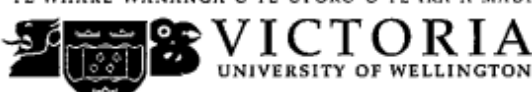

Information Sheet for a Study into Leadership Communication in Rugby

Researcher: Nick Wilson, School of Linguistics and Applied Language Studies, Victoria University of Wellington

\section{Introduction}

This study will investigate how language is used by players and coaches in rugby to carry out leadership and how this language use differs between professional and amateur rugby teams. The objective is to find out how players and coaches use language differently when rugby is their job, rather than a pastime. This research is part of the Wellington Language in the Workplace Project.

Language use will be analysed through observation and by audio recordings. It is hoped that the results of this study will be of use to rugby coaches and players in improving the way that they use communication techniques to be leaders, both on and off the field. The University requires that ethics approval be obtained for research involving human participants. As such you will be asked to give written consent to take part in this study.

\section{Recording}

Recordings will take place after a two month observation period to assess who would be
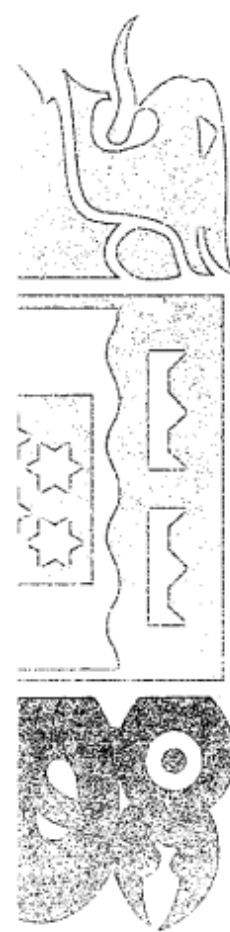
most suitable for recording. Two coaches and three players would be recorded from each team over three training sessions and two matches. All recordings will be made using small, digital recording devices. In the case of the players these will be attached in such a way as to minimise the inconvenience to the player and in the safest way possible. Any player who wears a device will be consulted about how it is to be worn and every effort will be made to ensure that the player is completely comfortable in wearing the device.

Participation in this study is voluntary, should you wish to withdraw from the study at any time you may do so and any of your recorded speech will be deleted. Although all members of the team will be observed only some will be recorded. Once all recordings have been made there will be feedback interviews conducted with the recorded participants, at which point they will have the opportunity to listen to what has been recorded and, if they wish, request that any part of the recording be deleted. There will also be the opportunity for further feedback meetings to discuss the results once the project nears completion.

SCHOOL OF LINGUISTICS \& APPLIED LANGUAGE STUDIES Te Kura Taatari Reo

incorporating

DEAF STUDIES RESEARCH UNIT ENGLISH LANGUAGE INSTITUTE NEW ZEALAND DICTIONARY CENTRE

PO Box 600 , Wellington, New Zealand

Phone $+64-4-4635600$ Fax +64-4-4635604 Email lals@vuw.ac.nz. Website www.victoria.ac.nz/lals 


\section{Appendix D: Ethics Approval}

\section{Confidentiality}

Upon the completion of the project all recorded data and transcriptions will be securely archived with the Wellington Language in the Workplace Project to which access is restricted to approved researchers. All data and notes, both written and recorded will only be available to the researcher and research supervisors for the duration of the research and will be securely stored at all times. In any written reports, articles and in the thesis, all names will be anonymised so as to protect the identities of the participants. Any sensitive information (e.g. lineout calls, strategies) will remain strictly confidential and will be scrambled in any written form of the data.

\section{Contact Details}

If you should have any questions or would like more information, please do not hesitate to contact me at nick.wilson@vuw.ac.nz or on 044635233 ext 8709, or contact my supervisors, Professor Janet Holmes and Dr Meredith Marra at the School of Linguistics and Applied Language Studies, Victoria University of Wellington, PO BOX 600 Wellington 6140, New Zealand, phone 044635600. 


\section{Leadership Communication in Rugby Consent Form}

I have been given and have understood an explanation of this research project. I have had an opportunity to ask questions and have them answered to my satisfaction. I understand that I may withdraw myself (or any information I have provided) from this project (before data collection and analysis is complete) without having to give reasons or without penalty of any sort.

I am aware that even if not selected to wear a microphone during the recording sessions there is still a chance that what I say may be recorded. If I wish not to be recorded I will alert the researcher to this fact and any part of the recording that features my voice will be deleted.

I understand that any information I provide will be kept confidential to approved researchers, the published results will not use my name, and that no opinions will be attributed to me in any way that will identify me. I understand that all audio recordings will be stored in the archives of the Wellington Language in the Workplace Project.

I wish to be informed of the results of the study and any recommendations relevant to me that can be made from it (tick if interested).

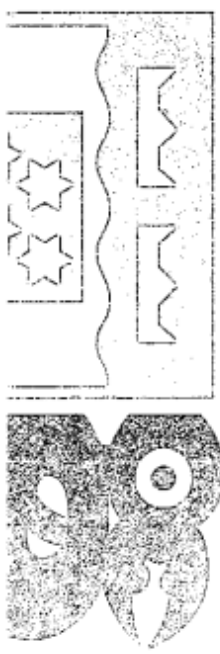

Signed:

Date:

Please print name:

SCHOOL OF LINGUISTICS \& APPLIED LANGUAGE STUDIES Te Kura Taatari Reo

incorporating

DEAF STUDIES RESEARCH UNIT ENGLISH LANGUAGE INSTITUTE NEW ZEALAND DICTIONARY CENTRE

PO Box 600 , Wellington, New Zealand

Phone +64-4-4635600 Fax +64-4-4635604 Email lals@vuw.ac.nz Website www.victoria.ac.nz/lals 